\title{
Health Technology Assessment in Epilepsy: Economic Evaluations and Preference Studies
}

Citation for published version (APA):

de Kinderen, R. J. A. (2015). Health Technology Assessment in Epilepsy: Economic Evaluations and Preference Studies. [Doctoral Thesis, Maastricht University]. Maastricht University. https://doi.org/10.26481/dis.20150522rk

Document status and date:

Published: 01/01/2015

DOI:

10.26481/dis.20150522rk

Document Version:

Publisher's PDF, also known as Version of record

\section{Please check the document version of this publication:}

- A submitted manuscript is the version of the article upon submission and before peer-review. There can be important differences between the submitted version and the official published version of record.

People interested in the research are advised to contact the author for the final version of the publication, or visit the DOI to the publisher's website.

- The final author version and the galley proof are versions of the publication after peer review.

- The final published version features the final layout of the paper including the volume, issue and page numbers.

Link to publication

\footnotetext{
General rights rights.

- You may freely distribute the URL identifying the publication in the public portal. please follow below link for the End User Agreement:

www.umlib.nl/taverne-license

Take down policy

If you believe that this document breaches copyright please contact us at:

repository@maastrichtuniversity.nl

providing details and we will investigate your claim.
}

Copyright and moral rights for the publications made accessible in the public portal are retained by the authors and/or other copyright owners and it is a condition of accessing publications that users recognise and abide by the legal requirements associated with these

- Users may download and print one copy of any publication from the public portal for the purpose of private study or research.

- You may not further distribute the material or use it for any profit-making activity or commercial gain

If the publication is distributed under the terms of Article $25 \mathrm{fa}$ of the Dutch Copyright Act, indicated by the "Taverne" license above, 


\section{Health Technology Assessment in Epilepsy}

Economic Evaluations and Preference Studies 
The studies presented in this thesis were conducted at the epilepsy center Kempenhaeghe and at the School for Public Health and Primary Care (CAPHRI), Department of Health Services Research (HSR) at Maastricht University.

The research presented in this thesis was funded by the Netherlands Organization for Health Research and Development (ZonMw) (project 171002204).

Printing and dissemination of this thesis was financially supported by CAPHRI HTA program, Cyberonics Europe BVBA, Epilepsiefonds, Kempenhaeghe, MHeNS, Nutricia Advanced Medical Nutrition, Pfizer B.V., and UCB Pharma B.V.

\section{ZonMw Kempenhaeghe $\oiint_{\text {De Macht van het Kleine }}^{\text {Epilepsiefonds }}$ caphri $\begin{aligned} & \text { School for Public Health } \\ & \text { and Primary Care }\end{aligned}$}

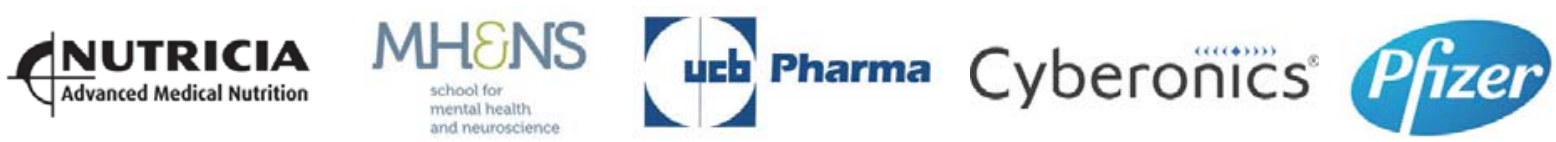

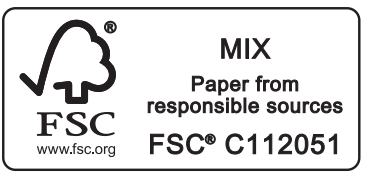

(C) Reina Van Bilsen - de Kinderen

Cover: Studio Bert Govers

Lay-out: Tiny Wouters

Printing: Ipskamp Drukkers

ISBN: 978-94-6259-675-7

No part of this thesis may be reproduced or transmitted in any form or by any means, electronic or mechanical, including photocopy, recording or otherwise without permission of the author. For all articles published or accepted the copyright has been transferred to the respective publisher. 


\title{
Health Technology Assessment in Epilepsy Economic Evaluations and Preference Studies
}

\author{
PROEFSCHRIFT \\ ter verkrijging van de graad van doctor \\ aan de Universiteit Maastricht \\ op gezag van de Rector Magnificus prof. dr. L.L.G. Soete, \\ volgens het besluit van het College van Decanen \\ in het openbaar te verdedigen \\ op vrijdag 22 mei 2015 om 14:00 uur
} door

Reina Johanna Adriana Van Bilsen - de Kinderen geboren op 12 januari 1986 te Geldrop 


\section{Promotores}

Prof. dr. mr. S.M.A.A. Evers

Prof. dr. A.P. Aldenkamp

\section{Copromotores}

Dr. H.J.M. Majoie

Dr. D. Postulart (Kempenhaeghe)

\section{Beoordelingscommissie}

Prof. dr. D. Ruwaard (voorzitter)

Prof. dr. P.A.J.M. Boon (Universiteit van Gent)

Dr. J. Nicolai

Dr. B.L.T. Ramaekers

Prof. dr. F. Smit (Trimbos Instituut / VU University Medical Centre) 



\section{Contents}

1 General introduction and outline of the thesis

$\begin{array}{llr}\text { PART I } & \text { Preferences and side-effects } & 21\end{array}$

2 From clinically relevant outcome measures to quality of life in

epilepsy: a time trade-off study

3 Eliciting patients' preferences for epilepsy diagnostics:

a discrete choice experiment

4 Side-effects of antiepileptic drugs: the economic burden

a methodological review

6 Research into the (cost-)effectiveness of the ketogenic diet among children and adolescents with intractable epilepsy: design of a randomized controlled trial

$7 \quad$ An economic evaluation of the ketogenic diet versus care as usual in children and adolescents with intractable epilepsy: an interim analysis

8 Cost-effectiveness of the ketogenic diet and vagus nerve stimulation for the treatment of children with intractable epilepsy

PART III Discussion and summary 



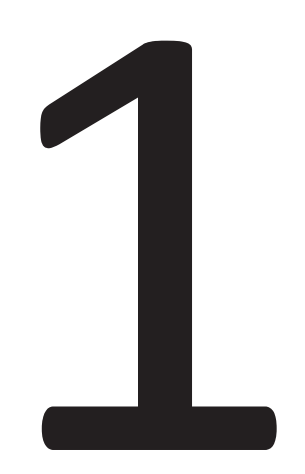

General introduction and outline of the thesis 
Chapter 1 


\section{General introduction}

Over the past decades, health care expenditure has been increasing rapidly in the Netherlands. Important cost drivers are the development and implementation of new, expensive medical interventions, intensification of treatment and care and rising cost prices. ${ }^{1}$ Since reducing health spending is high on the political agenda, policy makers are interested in the relative effectiveness and efficiency of medical interventions to support optimal priority-setting. Health Technology Assessment (HTA) is a field of research that aims to inform health policy makers in these decisions by examining the medical, economic, social and ethical implications of medical intervention. ${ }^{2}$

This thesis reports on HTA issues by focusing on several (methodological) challenges in the field of epilepsy and evaluating the cost-effectiveness of the ketogenic diet and the vagus nerve stimulator for children and adolescents with intractable epilepsy. This first chapter opens with some background information about epilepsy. The methodologies used in HTA research are explained hereafter, and finally, the outline and research questions are presented at the end of this chapter.

\subsection{Burden of epilepsy}

Epilepsy is a disease of the brain defined by any of the following conditions: I) At least two unprovoked (or reflex) seizures occurring >24 hours apart, II) one unprovoked (or reflex) seizure and a probability of further seizures similar to the general recurrence risk (at least 60\%) after two unprovoked seizures, occurring over the next 10 years, or III) diagnosis of an epilepsy syndrome. ${ }^{3}$ Epileptic seizures vary considerably in severity and frequency between patients, as well as in an individual patient. Some patients have multiple seizures per day, others only seldom. The clinical manifestations vary and depend on where in the brain the disturbance first starts and how far it spreads. The outward effect can be as dramatic as a wild thrashing movement, falling down unconscious or as mild as a brief loss of awareness. A seizure can be very prolonged, or very quick and short in succession. Status epilepticus can be life threatening, and is defined when a seizure lasts for more than 5 minutes or when the patient does not regain consciousness between two or more seizures.

Worldwide, about 65 million people are estimated to have epilepsy. ${ }^{4}$ In the Netherlands, the prevalence of active epilepsy is approximately $84,000 .^{5}$ Although difficult to estimate, epilepsy was considered to be the cause of death in 229 persons in the year 2010. ${ }^{6}$ Epilepsy mortality rates are related to young patients with severe intractable epilepsy in combination with other neurological problems and/or mental retardation. $^{7}$

Living with epilepsy can be a great burden for the patient himself and those around him. Patients with epilepsy heavily depend on informal care delivered by family and friends. Since epileptic seizures mostly occur unexpectedly, they may cause physical 
harm, as well as psychological stress due to their unpredictability. ${ }^{8}$ Epilepsy can sometimes result in significant disability, social exclusion and stigmatization. People with epilepsy commonly encounter problems in the following areas: education; employment; driving; personal development; psychiatric and psychological aspects and social and personal relationships. ${ }^{9,10}$

The annual total cost of epilepsy in Europe is $€ 15.5$ billion. ${ }^{11}$ In the Netherlands, direct medical costs were $€ 248$ million in 2011 , which is $0.3 \%$ of the health care budget. ${ }^{12}$ The main cost driver is health care professional visits and hospitalizations, accounting for almost half of the total direct medical costs (€122 million). Institutionalized patients are the second main cost driver, accounting for $€ 73.6$ million and the third main cost driver is the cost of medication (€36.5 million). ${ }^{12}$

\subsection{Treatment of epilepsy}

In the majority of patients, epilepsy is treatable with antiepileptic drugs (AEDs). About $30 \%$ of patients suffer from intractable epilepsy, indicating that their seizures are still uncontrolled after taking two or more different AEDs. ${ }^{13}$ Despite the availability of newer AEDs, the rates of intractability do not seem to have decreased and the likelihood of controlling seizures after two appropriately chosen AEDs fail, is low. ${ }^{14}$ Epilepsy surgery may be an option for people with focal seizures that remain a problem despite AEDs. Total seizure control can be achieved in $60-70 \%$ of the cases. ${ }^{15}$ For more than half the patients with intractable epilepsy, however, surgery is not an option. In this case, ketogenic diet (KD) and vagal nerve stimulation (VNS) are possible adjunctive options to AED therapy. ${ }^{16}$

The KD is a high-fat, low-carbohydrate diet that imitates the metabolic state of fasting while remaining normocaloric. Body energy requirements, while on the $K D$, are met by lipolysis and $\beta$-oxidation of fatty acids rather than by the breakdown of carbohydrates. ${ }^{17}$ VNS is a pacemaker-like device that is implanted subcutaneously into the upper part of the chest with a connecting wire running from the stimulator to an electrode that is attached to the vagus nerve. ${ }^{18}$ Once the stimulator is activated, electrical pulses will be generated at regular intervals depending on the anticonvulsant effect and on the patient's tolerance.

\subsection{Health Technology Assessment}

Health technology assessment (HTA) is a multi-disciplinary field of policy analysis that studies the medical, social, ethical and economic implications of development, diffusion, and use of health technology. ${ }^{2}$ HTA may address the direct and intended consequences of technologies as well as their indirect and unintended consequences. The objective is to provide a bridge between the world of research and the world of decision-making. ${ }^{19}$

A frequently used tool in HTA research is economic evaluation; this covers a range of types used to investigate the costs and consequences of interventions of programs, 
i.e. cost-benefit analysis (CBA), cost-effectiveness analysis (CEA) and cost-utility analysis (CUA). ${ }^{20}$

In $\mathrm{CBA}$, attempts are made to value the consequences of programs in monetary terms, in order to make them commensurate with the costs. Potentially, this is the broadest program for justifying the costs. Expressing benefits in monetary terms is, however, often hampered by measurement problems.

In CEA, the consequences of programs are measured in the most appropriate natural effects of physical units, such as 'seizure-free days' or 'seizure-free patients'. The main argument for these clinical measures is the relative ease of measurement and interpretation. One of the major limitations of the CEA and its disease-specific outcome is that it does not permit comparisons with interventions evaluated in other studies.

In CUA, the consequences of programs are adjusted by health state preference scores or utility weights; that is, states of health associated with the outcomes are valued relative to one another. The most common measure of consequences in a CUA is the quality-adjusted life-year (QALY).

In addition to the different types of economic evaluation mentioned above, economic evaluation can also be performed using different analytical frameworks, distinguishing trial-based economic evaluation from model-based economic evaluation. In trialbased economic evaluations, data on resource use and effectiveness are collected simultaneously, for example, alongside a randomized trial. The benefits of this approach are that results reflect actual data from real patients, and the internal validity of the data can be assessed through controlled clinical conditions. The disadvantage of this approach is that it may not be possible to generalize the treatment pathways and patient samples to the larger population. To overcome this disadvantage, it is possible to model the costs and outcomes of different treatments.

Model-based economic evaluations provide a means of bringing all clinical, costs and quality of life data together in one model by using techniques of epidemiology or decision analysis. Its result can, therefore, be generalized in terms of the larger population and lifetime costs and outcomes can then be estimated. Different models exist, decision-tree and state-transition models being the most commonly used. Decision-tree models present a sequence of decisions and chance events over time. Each chance event is assigned a probability. Alternative decision strategies are evaluated by calculating their average consequences. A limitation of decision-tree models is that the probability of each chance event is static. In chronic diseases, the probability of chance events changes with age, health status and time. State-transition Markov models are, therefore, often used; these allocate and reallocate subjects into previously defined health states. ${ }^{21}$ Transitions occur from one health state to another at defined time intervals according to the transition probabilities. Through simulation, the number of subjects in the population passing through each state at each point in time can be estimated, as well as the lifetime costs and effects. 


\subsection{Quality of life \& preferences}

There are no limits to the types of measures of effect that we can include in a costeffectiveness ratio. For our results to aid in decision-making, however, it is necessary to have a measure of effectiveness I) that allows direct comparison of therapies across a number of domains, II) that allows comparison across therapeutic areas and illnesses, and III) for which we have a general understanding of how much we are willing to pay for a unit of effect. ${ }^{22}$ Quality adjusted life year (QALY) is such a measure. A QALY incorporates effects in terms of both quality of life (utilities) and survival (lifeyears gained). To calculate QALYs, it is necessary to represent people's health state on a scale where death and full health are assigned values of 0 and 1 , respectively.

A common method for measuring a person's health state is through standardized questionnaires, on which participants can indicate how they score in different predefined health domains (e.g. "I can walk" to "I am bedridden"). A well-known instrument is the Euroqol-5D (EQ-5D) ${ }^{23}$, which measures health using five dimensions, namely: mobility, self-care, usual activities, pain/discomfort and anxiety/depression. Furthermore, all dimensions have three levels of functioning, namely: no problems, some problems and severe problems. Using such an instrument, a health state description is determined by a combination of the participants' answers. The unique combination of domains and levels leads to a certain number of health states; for example, the EQ-5D can distinguish 243 different health states.

The next step is to assign a value (utility) to the health state. The EQ-5D has utility values readily available in a so-called 'utility-tariff' for all 243 health states. ${ }^{24,25}$ The values of the utility-tariff are obtained from the general public through structured choice sets, based on the time trade-off (TTO) method. The TTO approach measures the preferences of the public for a certain health state. The method involves asking respondents to consider the relative amounts of time (life years) they would be willing to sacrifice to avoid a certain health state (i.e. a chronic condition like epilepsy with frequent seizures). Respondents may be indifferent given the choice between a certain health state and a shorter healthy lifetime of seven years, resulting in an estimated utility for the health state of 0.7 . Because the utility values attached to the health states reflect the preferences of people for such a state, instruments such as the EQ-5D are often referred to as preference-based instruments.

Not only can preferences be used for valuing health states, they can also be used to determine the most optimal treatment or test from the patient's point of view. Such preferences can be highly informative for decision-makers and clinicians. It has been advocated that the management of a disease like epilepsy should incorporate a shared decision-making process between doctor and patient. ${ }^{26,27}$ Shared decisionmaking is a process in which clinicians and patients work together to select test, treatments, management or support packages, based on clinical evidence and the patient's informed preferences. Within epilepsy treatment and diagnostics, there are many decisions which have no single 'best' choice. Such a decision is sensitive to preferences, because there is insufficient evidence or there is a need to trade-off 
known benefits and harms. ${ }^{28}$ Discrete choice experiments (DCE) can be used to reveal patients' stated preferences. A DCE is based on random utility theory and closely resembles actual decision-making; it is, therefore, a frequently used elicitation method in other areas of health to elicit patients' preferences. ${ }^{29-31}$

\subsection{When is an intervention cost-effective?}

There are several steps involved in determining whether or not a medical intervention is more cost-effective than usual care in economic evaluations. For a meaningful comparison, it is necessary to examine the additional costs that one medical intervention imposes over another, compared with the additional effects or utilities it delivers. In an incremental cost-effectiveness ratio (ICER), the additional costs and effects of intervention $A$ compared to $B$ are combined in the following formula ${ }^{22}$ :

$$
\frac{\text { Cost }_{A}-\text { Cost }_{B}}{\text { Effect }_{A}-\text { Effect }_{B}}=\frac{\Delta C}{\Delta E}=\frac{C}{E}
$$

The ICER can be illustrated graphically on a four quadrant diagram known as the costeffectiveness plane (see Figure 1.1). ${ }^{32}$ The south-east quadrant represents the instances where the intervention is more effective and less costly than the comparators (dominates), with the opposite situation in the north-west quadrant, representing interventions that are more costly and less effective than comparators (dominated). The north-east quadrant represents interventions that are more costly yet more effective, while the south-west quadrant represents less effective and less costly interventions. ${ }^{20}$

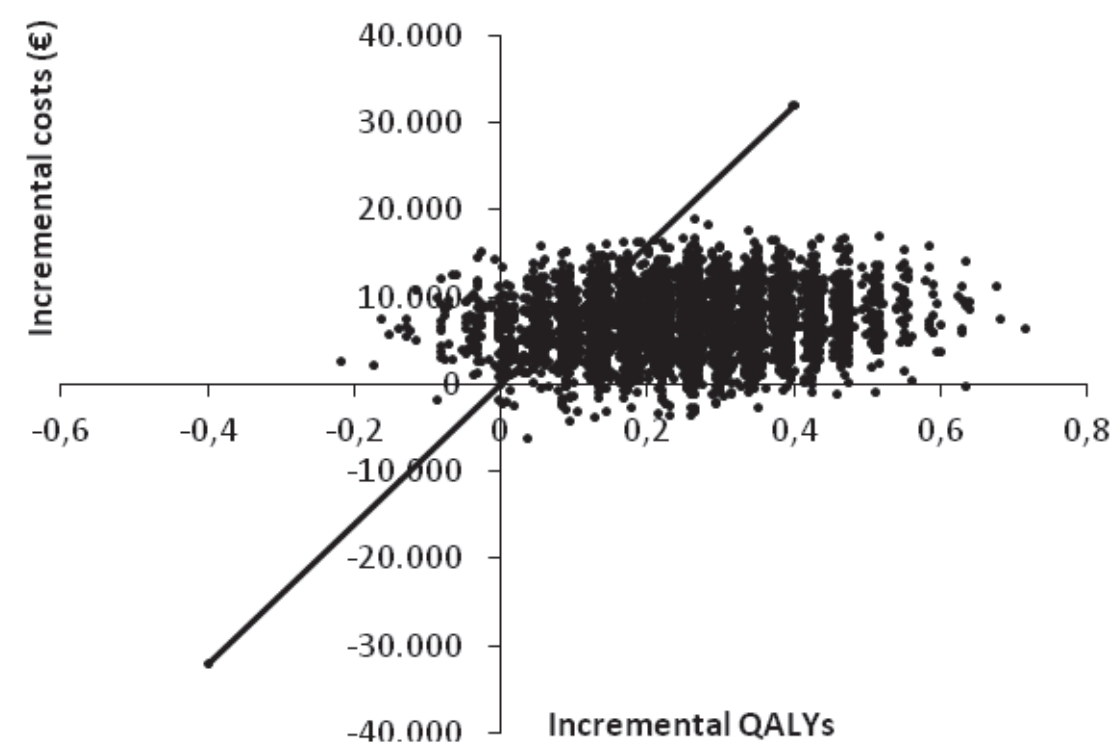

Figure 1.1 Cost-effectiveness plane. 
A treatment is deemed cost-effective when it is dominant or when the costs per QALY are below a specified ceiling ratio. In the Netherlands, an informal ceiling ratio between approximately $€ 20,000$ and $€ 80,000$ per QALY exists, depending on the burden of disease. ${ }^{33}$

Furthermore, cost-effectiveness acceptability curves (CEACs) can be calculated. ${ }^{20}$ CEACs show the probability ( $y$-axis) that a treatment is cost-effective, given different ceiling ratios ( $x$-axis) (see Figure 1.2).

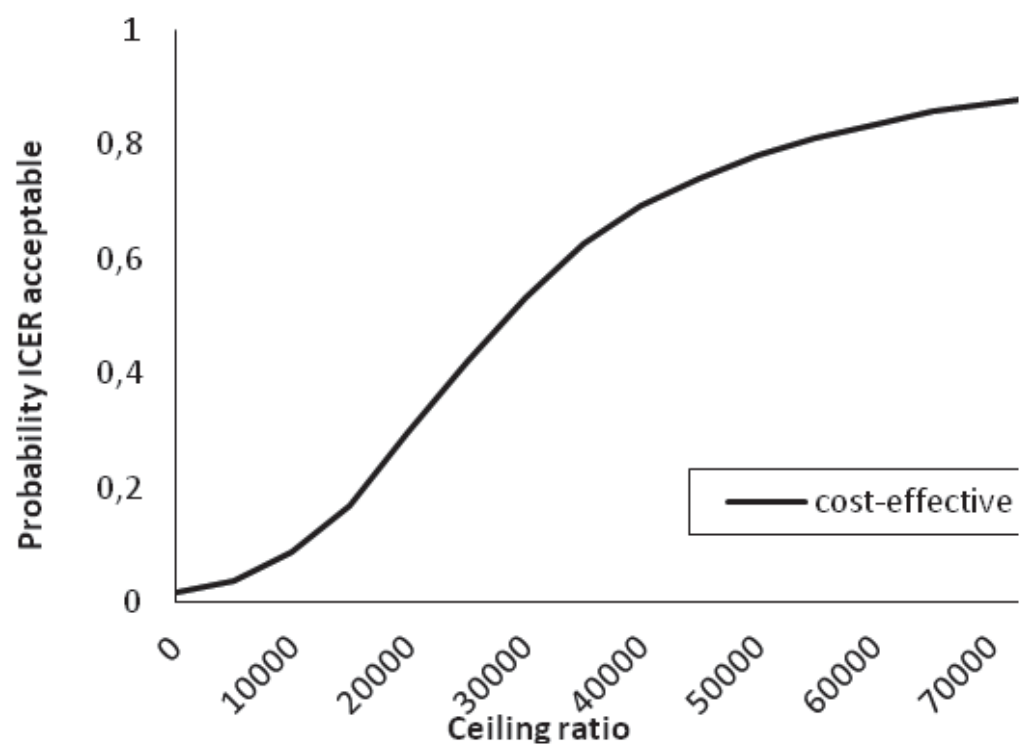

Figure 1.2 Cost-Effectiveness Acceptability Curves.

\subsection{HTA Challenges in epilepsy research}

Many present-day scientific and clinical challenges in the field of epilepsy may benefit from multidisciplinary cooperation between clinicians and HTA researchers. Although both stakeholders aim at evidence-based decision-making, they use different methodologies and outcomes to achieve this common aim. The first challenge is to 'merge' HTA outcome measures with common clinical outcome measures in epilepsy. Usually, clinical studies measure the effect of interventions with common clinical measures (e.g. seizure frequency and side-effects) ${ }^{34-38}$, rather than quality of life measures or (patient) preferences. Secondly, although side-effects of AEDs are frequently researched ${ }^{39-41}$, their economic impact is still largely unknown. ${ }^{42}$ Furthermore, full economic evaluations of the KD and VNS, and direct comparisons of both interventions are lacking in the literature which makes it difficult for clinicians and HTA researchers to determine the most optimal treatment. In addition, due to scarcity of resources, it is increasingly important to examine whether benefits outweigh costs. The general aim of this thesis is, therefore, two-fold. The first aim is to 
address a number of methodological issues concerning outcome assessment. The second aim is to estimate the costs, benefits and incremental cost-effectiveness of the KD and VNS as treatment options for children with intractable epilepsy.

\subsection{Research questions and outline of this thesis}

Part I of this thesis investigates some of the methodological challenges of HTA research in the field of epilepsy, in relation to economic aspects and preferences. In part II of this thesis, economic evaluations are applied in epilepsy research and the cost-effectiveness of treatments for intractable epilepsy are researched. The research questions addressed in this thesis are formulated below.

\section{Part I - Preferences and side-effects}

Research question 1: What are the preferences for outcome measures and diagnostics in epilepsy?

Although the use of the QALY is widespread, there is little scientific consensus about methods used relating to the description and valuation based on preferences of health states. The debate relates to questions such as who should value health, how is health to be valued, and how should health be described? Furthermore, another practical issue related to this debate is the estimation of QALYs when utilities are not measured in a study. Chapter $\mathbf{2}$ contributes to this discussion by measuring preferences of the general population for three clinical outcome measures in epilepsy research (e.g. seizure frequency, seizure severity and side-effects).

In an era where shared decision making and patient autonomy are highly valued, it is argued that including patient preferences in research is indispensable. Chapter 3 investigates whether preferences for diagnostic properties can be measured with a discrete choice experiment. Patients were led through a stated preferences experiment in which features of different diagnostic modalities (e.g. EEG and MEG) were presented.

Research question 2: What is the economic burden of side-effects due to AEDs in epilepsy?

Antiepileptic drugs (AEDs) are the most used therapy for epilepsy; there are (theoretically) approximately 25 different AEDs available for epilepsy patients. Although the AEDs are, in most patients, very effective in reducing the frequency of seizures, they are accompanied by (subjective) side-effects in approximately $25 \%$ of the patients. The treatment of these side-effects can range from minor care to longterm hospitalizations. Chapter $\mathbf{4}$ investigates the economic burden of the side-effects related to AEDs. 


\section{Part II - Economic evaluations}

Research question 3: What are cost-effective options in the treatment of intractable epilepsy?

Patients who suffer from intractable epilepsy continue to have seizures after taking two or more different AEDs. When newly developed AEDs appear on the market, patients with intractable epilepsy can potentially benefit. Despite the availability of newer AEDs and the option of epilepsy surgery, many patients with intractable epilepsy will not respond to these medications or appear not to be eligible for surgery. The ketogenic diet and the vagus nerve stimulator are two other treatment options. Chapter $\mathbf{5}$ gives an overview of the cost-effectiveness of treatments for patients with intractable epilepsy. A systematic review is performed in order to retrieve all available evidence from the current body of literature.

During our research described in chapter 5, no economic evaluations were found on the ketogenic diet. In order to investigate the cost-effectiveness of the ketogenic diet compared to care as usual, a randomized controlled trial was initiated. This so-called KOEK-study (KOsten Effectiviteit Ketogeen dieet) investigated the effects and costs of the diet compared to care as usual in children and adolescents with intractable epilepsy. A full description of the randomized controlled study design, outcomes used and methods applied is given in chapter 6 .

In chapter 7, the interim results of the trial-based economic evaluation of the KOEKstudy are presented. Due to slow patient inclusion, a full, long-term evaluation of the cost-effectiveness did not seem to be feasible during the time of writing this thesis. The cost-effectiveness results based on the data of 48 children and adolescents, gathered during the first 4-months of the KOEK-study, are summarized in chapter 7.

As the ketogenic diet and vagus nerve stimulator are both potentially effective treatments for patients with intractable epilepsy, we aimed to compare the two therapies in a long-term economic model. A direct comparison of, and economic information on these therapies is lacking in the literature. The model-based economic evaluation of the ketogenic diet, vagus nerve stimulation and care as usual is described in chapter 8.

\section{Part III - Discussion and summary}

Finally, chapter 9 provides answers to the research questions and discusses the merits and limitations of the research performed in this thesis. A summary of the thesis is provided in chapter $\mathbf{1 0}$ and one can find a Dutch summary of the thesis in chapter 11. Note that chapters 2-8 are based on publications in (or intended for) international peer reviewed journals and can thus be read independently. 


\section{References}

1. Slobbe LCJ, Smit JM, Groen J, Poos MJJC, Kommer GJ. Kosten van ziekten in Nederland 2007: Trends in de Nederlandse zorguitgaven 1999-2010. Bilthoven: RIVM; 2011.

2. Hailey D, Babidge W, Cameron A, Davignon A. An INAHTA guidance document: INAHTA; 2010 [cited 2014 18-10]. Available from: http://www.inahta.org/wp-content/themes/inahta/img/ HTA\%20\%Decision\%20Makers.pdf.

3. Fisher RS, Acevedo C, Arzimanoglou A, Bogacz A, Cross JH, Elger CE, Engel J Jr, Forsgren L, French JA, Glynn M, Hesdorffer DC, Lee BI, Mathern GW, Moshé SL, Perucca E, Scheffer IE, Tomson T, Watanabe M, Wiebe S. ILAE official report: a practical clinical definition of epilepsy. Epilepsia. 2014;55:475-82.

4. Ngugi AK, Bottomley C, Kleinschmidt I, Sander JW, Newton CR. Estimation of the burden of active and life-time epilepsy: a meta-analytic approach. Epilepsia. 2010;51:883-90.

5. Kotsopoulos IA, Evers SM, Ament AJ, de Krom MC. Estimating the costs of epilepsy: an international comparison of epilepsy cost studies. Epilepsia. 2001;42:634-40.

6. Statistiek CBvd. Doodsoorzakenstatistiek: CBS; 2010 [cited 2014 18-10]. Available from: http://www.cbs.nl/nl-NL/menu/methoden/dataverzameling/doodsoorzakenstatistiek.htm.

7. Klenerman P, Sander JW, Shorvon SD. Mortality in patients with epilepsy: a study of patients in long term residential care. J Neurol Neurosurg Ppsychiatry. 1993;56:149-52.

8. Elliott JO, Richardson VE. The biopsychosocial model and quality of life in persons with active epilepsy. Epilepsy Behav. 2014;41C:55-65.

9. Suurmeijer TP, Reuvekamp MF, Aldenkamp BP. Social functioning, psychological functioning, and quality of life in epilepsy. Epilepsia. 2001;42:1160-8.

10. Kobau R, Cui W, Kadima N, Zack MM, Sajatovic M, Kaiboriboon K, Jobst B. Tracking psychosocial health in adults with epilepsy-Estimates from the 2010 National Health Interview Survey. Epilepsy Behav. 2014;41C:66-73.

11. Pugliatti M, Beghi E, Forsgren L, Ekman M, Sobocki P. Estimating the cost of epilepsy in Europe: a review with economic modeling. Epilepsia. 2007;48:2224-33.

12. Kosten van ziekten in Nederland 2011. Bilthoven: RIVM; 2011.

13. Sillanpaa M, Schmidt D. Natural history of treated childhood-onset epilepsy: prospective, long-term population-based study. Brain. 2006;129:617-24.

14. Kwan P, Brodie MJ. Early identification of refractory epilepsy. N Engl J Med. 2000;342:314-9.

15. Duncan JS, Sander JW, Sisodiya SM, Walker MC. Adult epilepsy. Lancet. 2006;367:1087-100.

16. Sheth RD, Stafstrom CE. Intractable pediatric epilepsy: vagal nerve stimulation and the ketogenic diet. Neurologic clinics. 2002;20:1183-94.

17. Freeman J, Veggiotti P, Lanzi G, Tagliabue A, Perucca E. The ketogenic diet: from molecular mechanisms to clinical effects. Epilepsy Res. 2006;68:145-80.

18. Ben-Menachem E1, Mañon-Espaillat R, Ristanovic R, Wilder BJ, Stefan H, Mirza W, Tarver WB, Wernicke JF. Vagus nerve stimulation for treatment of partial seizures: 1 . A controlled study of effect on seizures. First International Vagus Nerve Stimulation Study Group. Epilepsia. 1994;35:616-26.

19. Battista RN, Hodge MJ. Quality of life research and health technology assessment--a time for synergy. Qual Life Res. 1996;5:413-8.

20. Drummond MF, Sculpher MJ, Torrance GW, O'Brien BJ, Stoddart GL. Methods for the economic evaluation of health care programs. Oxford: Oxford University Press; 2005.

21. Briggs A, Claxton K, Sculpher MJ. Decision Modellling for Health Economic Evaluation. Gray A, Briggs A, editors. Oxford: Oxford University Press; 2006.

22. Glick HA, Doshi JA, Sonnad SS, Polsky D. Economic Evaluation in Clinical Trials. Oxford: Oxford university press; 2007.

23. EuroQol G. EuroQol--a new facility for the measurement of health-related quality of life. The EuroQol Group. Health Policy. 1990;16:199-208.

24. Dolan P. Modeling valuations for EuroQol health states. Med Care. 1997;35:1095-108.

25. Lamers LM, McDonnell J, Stalmeier PF, Krabbe PF, Busschbach JJ. The Dutch tariff: results and arguments for an effective design for national EQ-5D valuation studies. Health Econ. 2006;15: 1121-32. 
26. Coulter A, Entwistle V, Gilbert D. Sharing decisions with patients: is the information good enough? BMJ. 1999;318:318-22.

27. Holmes-Rovner M, Llewellyn-Thomas H, Entwistle V, Coulter A, O'Connor A, Rovner DR. Patient choice modules for summaries of clinical effectiveness: a proposal. BMJ. 2001;322:664-7.

28. Stacey D1, Légaré F, Col NF, Bennett CL, Barry MJ, Eden KB, Holmes-Rovner M, Llewellyn-Thomas $H$, Lyddiatt A, Thomson R, Trevena L, Wu JH. Decision aids for people facing health treatment or screening decisions. Cochrane Database Syst Rev. 2014;1:CD001431.

29. Bryan S, Buxton M, Sheldon R, Grant A. Magnetic resonance imaging for the investigation of knee injuries: an investigation of preferences. Health Econ. 1998;7:595-603.

30. Brown DS, Poulos C, Johnson FR, Chamiec-Case L, Messonnier ML. Adolescent girls' preferences for HPV vaccines: a discrete choice experiment. Adv Health Econ Health Serv Res. 2014;24:93-121.

31. Hiligsmann M, Dellaert BG, Dirksen CD, van der Weijden T, Goemaere S, Reginster JY, Watson V, Boonen A. Patients' preferences for osteoporosis drug treatment: a discrete-choice experiment. Arthritis Res Ther. 2014;16:R36.

32. Black WC. The CE plane: a graphic representation of cost-effectiveness. Med Decis Making. 1990;10: 212-4.

33. Care $\mathrm{CfPHaH}$. Sensible and sustainable care (in Dutch). Zoetermeer: Council for Public Health and Health Care; 2006.

34. Privitera MD, Welty TE, Ficker DM, Welge J. Vagus nerve stimulation for partial seizures. Cochrane Database Syst Rev. 2002 (1):CD002896.

35. Levy RG, Cooper PN, Giri P. Ketogenic diet and other dietary treatments for epilepsy. Cochrane Database Syst Rev. 2012;3:CD001903.

36. Michael B, Marson AG. Clobazam as an add-on in the management of refractory epilepsy. Cochrane Database Syst Rev. 2008 (2):CD004154.

37. Jette N, Hemming K, Hutton JL, Marson AG. Topiramate add-on for drug-resistant partial epilepsy. Cochrane Database Syst Rev. 2008 (3):CD001417.

38. Chadwick DW, Marson AG. Zonisamide add-on for drug-resistant partial epilepsy. Cochrane Database Syst Rev. 2005 (4):CD001416.

39. Thome-Souza S, Kadish NE, Ramgopal S, Sánchez Fernández I, Bergin AM, Bolton J, Harini C, Libenson M, Olson H, Peters J, Poduri A, Rotenberg A, Takeoka M, Kothare SV, Kapur K, Bourgeois BF, Loddenkemper T. Safety and retention rate of rufinamide in 300 patients: a single pediatric epilepsy center experience. Epilepsia. 2014;55:1235-44.

40. Zaccara G, Almas M, Pitman V, Knapp L, Posner H. Efficacy and safety of pregabalin versus levetiracetam as adjunctive therapy in patients with partial seizures: a randomized, double-blind, noninferiority trial. Epilepsia. 2014;55:1048-57.

41. Krauss GL, Perucca E, Ben-Menachem E, Kwan P, Shih JJ, Clément JF, Wang X, Bagul M, Gee M, Zhu J, Squillacote D. Long-term safety of perampanel and seizure outcomes in refractory partial-onset seizures and secondarily generalized seizures: results from phase III extension study 307 . Epilepsia. 2014;55:1058-68.

42. Schlienger RG, Oh PI, Knowles SR, Shear NH. Quantifying the costs of serious adverse drug reactions to antiepileptic drugs. Epilepsia. 1998;39 Suppl 7:S27-32. 


\section{Part}

Preferences and side-effects 


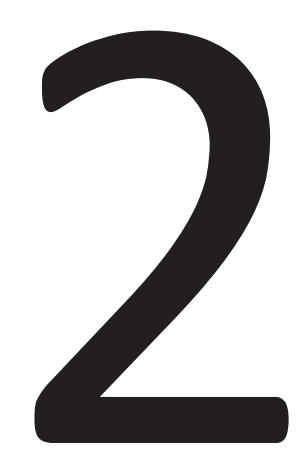

\section{From clinically relevant outcome measures to quality of life in epilepsy: a time trade-off study}

RJA de Kinderen, BFM Wijnen, G van Breukelen, D Postulart, HJM Majoie, AP Aldenkamp, SMAA Evers Submitted to Value in Health 


\section{Abstract}

\section{Objectives}

A proposed method for bridging the gap between clinically relevant epilepsy outcome measures and QALYs is to derive utility scores for epilepsy health states. The aim of this study is to develop such a utility-function and to investigate the impact of the epilepsy outcome measures on utility.

\section{Methods}

Health states, based on clinically important epilepsy attributes (e.g. seizure frequency, seizure severity, side-effects), were valued by a sample of the Dutch population $(\mathrm{N}=525)$ based on the time trade-off method. In addition to standard demographics, every participant was asked to rate 10 or 11 different health state scenarios. A multilevel regression analysis was performed to account for the nested structure of the data.

\section{Results}

Results show that the best health state (no seizures and no side-effects) is estimated at 0.89 and the worst state (seizures type 5 twice a day plus severe side-effects) at 0.22 (scale: 0-1). An increase in seizure frequency, occurrence of side-effects, and seizure severity were all significantly associated with lower utility values. Furthermore, seizure severity has the largest impact on quality of life compared with seizure frequency and side-effects.

\section{Conclusions}

This study provides a utility-function for transforming clinically relevant epilepsy outcome measures into utility estimates. We advise using our utility-function in economic evaluations, when quality of life is not directly measured in a study and hence, no health state utilities are available, or when there is convincing empirical evidence of the insensitivity of a generic QoLinstrument within epilepsy. 


\section{Introduction}

Quality of life (QoL) is a very important outcome measure, not only for patients, but also as an input for cost-utility analyses. However, the essential utilities are not always available. Especially in case of model-based, cost-utility analyses, where researchers are dependent on the published literature, suitable utilities for certain health states are often hard to find.

Problems in gathering utility values may occur because clinical trials and clinical evaluations measure the effects of interventions with common clinically relevant measures, rather than with QoL-measures. This also seems to be the case in epilepsy research.

Epilepsy is a brain disorder which is characterized by recurrent seizures. A seizure is a temporary symptom of abnormal excessive neuronal activity in the brain. ${ }^{1}$ The outward effect can be as dramatic as a wild, thrashing movement and falling to the ground unconscious, or as mild as a brief loss of awareness. Within the field of epilepsy, three disease-specific clinical outcome measures are often used to reflect patients' health, namely; seizure frequency, seizure severity and side-effects due to antiepileptic drugs (AEDs). Frequency and severity of seizures in people with epilepsy vary from one individual to another. In some people, seizures are very severe and occur frequently despite treatment with AEDs. In others, seizures are mild, less frequent and more easily controlled by AEDs. Seizure severity is measured using standardized questionnaires. One of the most frequently used seizure severity questionnaires focusing on the clinical events of a seizure, is the National Hospital Seizure Severity Scale (NHS3), a revised and simplified version of the Chalfont scale. ${ }^{2}$ In order to bridge the gap between clinical trials, which measure clinical outcomes, and economic evaluations, which require utility values, it would be preferable if one could make clinically relevant epilepsy outcome measures applicable to cost-utility analysis by deriving utility scores for the epilepsy outcome measures. For this purpose, a commonly used method is the time trade-off method (TTO). ${ }^{3}$ This has been used before in four other studies which elicited utilities for epilepsy health states. Firstly, Maltoni and Messori (1998) interviewed a small series of refractory epilepsy patients $(\mathrm{N}=81)$ in order to classify the patient according to five health states ('lamotrigine withdrawal due to serious side-effects', 'no response', 'partial response of at least $50 \%$ seizure reduction with persistent seizures', 'nearly complete response of at least $50 \%$ seizure reduction', and 'complete response with total freedom from seizures'). Then patients were asked how much of their current state of health they would be willing to give up in order to live the remaining years in excellent health. ${ }^{4}$ Secondly, Forbes et al. (2003) executed a TTO experiment among epilepsy patients $(\mathrm{N}=43)$ in order to assess patients' own health state and their health state with a $50 \%$ reduction in seizures; only seven patients understood the exercise. ${ }^{5}$ Thirdly, Carroll and Downs (2009) described utilities for several disease-related health states, including epilepsy. ${ }^{6}$ They interviewed parents $(\mathrm{N}=4016)$ to obtain their assessment of 
various health states ('mild seizure disorder, 'moderate seizure disorder' and 'severe seizure disorder') while imagining that it would be one of their children who was experiencing the health state under consideration. Lastly, Kang et al. (2014) asked the general population $(\mathrm{N}=300)$ also to assess three epilepsy health states ('Seizure-free', ' $50 \%$ or more seizure reduction', and 'less than $50 \%$ seizure reduction'). ${ }^{7}$ These published TTO-studies elicited utilities for a very small number of specific health states (i.e. 1-5); furthermore, in these studies, seizure severity and side-effects were not (fully) included in the health state description.

To overcome this gap, the objective of this study is twofold: first of all, to create a reliable utility-function to transfer clinically relevant epilepsy outcomes to health state utilities based on preferences of the general public measured with the TTO method, and secondly, to investigate the impact of the separate clinically relevant outcome measures on health state utility.

\section{Methods}

\section{Attributes and levels}

Health states were developed based on three clinically important disease-specific outcome measures (i.e. attributes): seizure frequency, seizure severity and treatmentrelated side-effects. These attributes were selected based on expert advice and commonality of use within the clinical epilepsy setting. Table 2.1 presents the three attributes and their levels. The attribute "seizure frequency" comprises 6 levels ranging from no seizures to two seizures per day. The levels of "seizure severity" were based on the description of seizures used in the valuation study of the National Hospital Seizure Severity Scale-3 (NHS3). ${ }^{2}$ The attribute "experience of side-effects" was categorized into three levels: no or mild, moderate and severe side-effects. Figure 2.1 presents an example of a health state to be valued by participants.

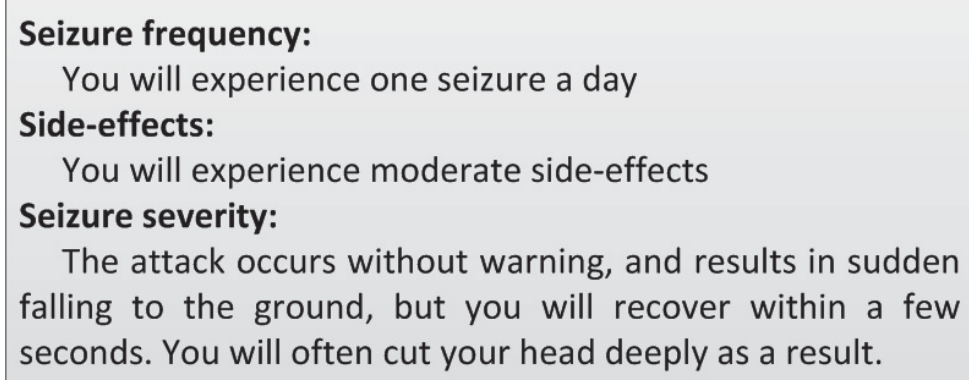

Figure 2.1 Example of epilepsy health state description. 
Table 2.1 Attributes and levels for TTO exercise.

\begin{tabular}{|c|c|c|}
\hline Attributes & (coding) Levels & $\begin{array}{l}\text { Regression } \\
\text { coefficient }\end{array}$ \\
\hline \multirow{6}{*}{$\begin{array}{l}\text { Seizure } \\
\text { frequency }\end{array}$} & No seizures & Redundant $^{\top}$ \\
\hline & Once a month & Reference \\
\hline & Once a week & $\beta 1$ \\
\hline & Twice a week & $\beta 2$ \\
\hline & Once per day & $\beta 3$ \\
\hline & Twice per day & $\beta 4$ \\
\hline $\begin{array}{l}\text { Experience of } \\
\text { side-effects }\end{array}$ & $\begin{array}{l}\text { No to mild side-effects } \\
\text { Minor side-effects } \\
\text { Severe side-effects }\end{array}$ & $\begin{array}{c}\text { Reference } \\
\beta 5^{*} \\
2 \beta 5^{*}\end{array}$ \\
\hline \multirow{5}{*}{$\begin{array}{l}\text { Seizure severity } \\
\text { description }\end{array}$} & $\begin{array}{l}\text { Type 1: The attack consists of a } 10 \text {-second blank spell during which the } \\
\text { patient stares straight ahead. The recovery is immediate and there are } \\
\text { no after-effects. There are no falls or injuries. }\end{array}$ & Reference \\
\hline & $\begin{array}{l}\text { Type } 2 \text { : The attack starts with a fluttering feeling in the stomach, which } \\
\text { warns the patient to sit or lie down. The patient then loses awareness for } \\
1 / 2 \text { a minute during which he smacks his lips. When the attack is over the } \\
\text { patient is back to normal within } 10 \text { minutes. }\end{array}$ & $\beta 6$ \\
\hline & $\begin{array}{l}\text { Type 3: The attack occurs without warning, and results in sudden falling } \\
\text { to the ground, but the patient recovers within a few seconds. The patient } \\
\text { often cuts his head deeply as a result. }\end{array}$ & $\beta 7$ \\
\hline & $\begin{array}{l}\text { Type 4: The attack starts without warning, and begins with the patient } \\
\text { becoming confused, during which he may act oddly like undressing } \\
\text { himself or moving objects around; occasionally he is incontinent for urine } \\
\text { or falls to the ground. He has never injured himself. The patient then } \\
\text { comes round, and the recovery normally takes } 30 \text { minutes. }\end{array}$ & $\beta 8$ \\
\hline & $\begin{array}{l}\text { Type 5: The attack starts without warning, and the patient always falls } \\
\text { unconscious to the floor, and then has a "grand mal" convulsion (with } \\
\text { shaking of the arms and legs). The patient is often incontinent for urine } \\
\text { and always bites his tongue. Full recovery takes } 6 \text { hours. }\end{array}$ & $\beta 9$ \\
\hline \multirow[t]{2}{*}{ Seizure-free } & No & Reference \\
\hline & Yes & $\beta 10$ \\
\hline
\end{tabular}

'Level "No seizures" is captured by the extra dummy indicator "Seizure-free" coded as 0, not seizure-free; 1 , seizure-free. ${ }^{*}$ Included in the model as a continuous variable.

\section{Valuation exercise}

Preferences of a sample of the general population were elicited through a time tradeoff (TTO) exercise for each of the epilepsy health states. The TTO was based on a process of outward titration to select a length of time in a health state with full health which they regarded as being equivalent to 10 years in an epilepsy health state. In other words; participants were asked which life they thought would be better, life $A$ or life B (or are the two equivalent)? Life A: living for 10 years within the presented epilepsy health state; life $B$ : living $t=$ years in perfect health. In the case that the participant prefers life $A$ to life $B, t$ is increased by 1 point (maximum $t=10$ ), until the participant becomes indifferent to whether it is life $A$ or life $B$. The other way around, if a participant prefers life $B$ to life $A, t$ is decreased (minimum $t=0$ ), until the participant becomes indifferent to whether it is life $A$ or life $B$. The smaller the 
'equivalent' number ' $\mathrm{t}$ ', the worse the epilepsy health state. If a respondent was willing to sacrifice all $t$ years in full health to avoid a certain epilepsy health state, then the participant valued the epilepsy health state as being equivalent to death.

A (nearly) full factorial design was used which resulted, after removing all unrealistic scenario's (i.e. all combinations of "no seizures" with "seizure type"), in 78 different epilepsy health states. Each participant valued a subset of 10 or 11 epilepsy health states, comprising the mildest possible health state (no seizures and no to mild sideeffects), the worst possible health state (i.e. two seizures per day, severe side-effects and seizure description 5) and a random subset of 8 or 9 relatively mild, moderate and severe health states.

\section{Questionnaire}

The questionnaire was self-administered using online survey software Qualtrics, version 2013 (Qualtrics, Provo, UT, USA). The first part of the online questionnaire consisted of socio-economic and background questions/characteristics: age, gender, educational level and experience with epilepsy (e.g. friend or family with epilepsy). Furthermore, we asked patients whether they believe in life after death, as this might influence their willingness to trade-off life years in the valuating exercise. ${ }^{8}$ Lastly, participants were asked to describe their own QoL using the EuroQol-5 Dimensions $(E Q-5 D)^{9}$ including a Visual Analogue Scale (VAS). The second part of the questionnaire started with background information about epilepsy and quality of life. Next, the attributes and levels of epilepsy health states were explained and some example questions were shown as a warm-up exercise for the TTO. The third and final part of the questionnaire consisted of the TTO exercises and questions about the time required by the participants to complete the questionnaire and their experience regarding the difficulty of the TTO exercise.

\section{Study participants}

Participants from the general population aged $>18$ years without epilepsy were recruited using e-mail, personal communication and social media. A link was provided to participants, which gave access to the online questionnaire. As part of this communication, we asked the participants to forward our invitation to participate in the study to their family and friends (again aged $>18$, without epilepsy). Participants who did not fully complete the questionnaire were excluded from the analyses. Participants were told that if they decided to complete the questionnaire, they thereby consented to participate in the study. In addition, information was provided that participation, and/or forwarding the invitation to friends and family, was completely voluntary and anonymous. This consent procedure was approved by the medical ethical committee of the epilepsy center Kempenhaeghe. 


\section{Analyses}

Utilities (U) were derived by determining the number of life years one is willing to sacrifice in order to become indifferent with regard to the choice between the epilepsy health state $(\mathrm{H})$ scenario $(\mathrm{j})$ to be valued and $t$ years spent in full health. Afterwards, $U$ is determined as $U\left(H_{j}\right)=t / 10$, where $t$ is the time in full health.

To account for the nested structure of the data (i.e. each respondent evaluated 10 or 11 scenarios), multilevel analyses were performed to develop the best model for the effects of the three attributes (seizure frequency, seizure severity, side-effects) on utility, thereby also to develop the utility-function, and to determine the effect of other covariates on the health state valuation of the respondents. We started with a random intercept model to capture inter-individual differences plus an ARMA autocorrelated errors model for the unexplained intra-individual response variation throughout the trials. To find the best model for the attribute effects on the utility, several models were compared by likelihood ratio (LR) testing, using maximum likelihood ( $M L$ ) estimation, since $M L$ estimation is required for $L R$ tests of models differing in their fixed/predictor part. ${ }^{10}$ However, the final model was re-estimated using restricted maximum likelihood (REML) estimation to obtain unbiased estimates of standard errors. ${ }^{10}$

Subsequently, to determine whether participants' religious belief, age, gender, educational level, experience with epilepsy (i.e. a close relative or a friend with epilepsy) and current QoL (EQ-5D and VAS score) significantly impacted the valuation exercise, they were included in the model as covariates. Multicollinearity of the covariates was assessed by checking if the Variance Inflation Factor (VIF) was below 10 for every covariate.

\section{Additional analyses}

We used the same model for the additional analyses, but now incorporating the utility values derived with the lead time trade-off. ${ }^{11}$ When participants traded off all 10 healthy life years to avoid a certain health state, they were offered the possibility of valuing the health state as being worse than death (SWD). In this case, the same scenario was presented using the lead time trade-off approach in which the respondent was asked to select a length of time $(t)$ for which one is indifferent between spending $t$ years in full health or spending 5 years in full health followed by 10 years in the given health state $\left(\mathrm{H}_{\mathrm{j}}\right)$. The corresponding utility is then estimated as $U\left(H_{j}\right)=(t-5) /(15-5)$, resulting in a range of -0.5 to 0 for states worse than death. For all health states, if the participant did not trade off all healthy life years, the utility calculation remained unchanged. 


\section{Results}

\section{Background characteristics}

In total, 529 subjects from the general population participated in the TTO study. Four participants were excluded from the analyses as their data were incomplete due to a computer error. Therefore, a total of 525 participants were included in the analyses. The mean age of the included sample was 43 years; more than half $(59.5 \%)$ were women; most of them had an educational level of at least secondary vocational education $(86.1 \%)$ and $61.9 \%$ had had no experience with epilepsy whatsoever. They valued their own health state, based on the EQ-5D at 0.86 (Dutch tariffs) ${ }^{8}, 0.84$ (UK tariffs) ${ }^{12}$ and 84.7 (VAS). Of the included participants, $37 \%$ indicated that they believed in life after death (Table 2.2).

Table 2.2 Respondent characteristics.

\begin{tabular}{|c|c|c|}
\hline Characteristics & Total cohort $\% \quad(\mathrm{~N}=525)$ & General Dutch population $^{++}$ \\
\hline \multicolumn{3}{|l|}{ Gender } \\
\hline Male & 40.5 & 49.5 \\
\hline Female & 59.5 & 50.5 \\
\hline \multicolumn{3}{|l|}{ Age in years } \\
\hline $20-40$ & $44.9^{i}$ & 24.6 \\
\hline $40-65$ & 45.4 & 35.5 \\
\hline $65-80$ & 9.7 & 12.6 \\
\hline $80+$ & 0 & 4.2 \\
\hline \multicolumn{3}{|l|}{ Education } \\
\hline No education & 0.4 & 1 \\
\hline Primary school & 1.3 & 8 \\
\hline Pre-vocational secondary school & 12.2 & 19 \\
\hline Secondary school ${ }^{*}$ & 30.2 & 40 \\
\hline Higher education $^{* *}$ & 55.9 & 32 \\
\hline \multicolumn{3}{|l|}{ Experience with epilepsy } \\
\hline No experience & 61.9 & N.a. \\
\hline Relative with epilepsy & 10.1 & N.a. \\
\hline Friend with epilepsy & 4.8 & N.a. \\
\hline Former epilepsy patient & 0.8 & N.a. \\
\hline Other & 22.5 & N.a. \\
\hline \multicolumn{3}{|l|}{ Believes in life after death } \\
\hline Yes & 37.0 & N.a. \\
\hline No & 63.0 & N.a. \\
\hline \multicolumn{3}{|l|}{ Mean EuroQol-5D score } \\
\hline Dutch tariffs & 0.86 & $0.91^{+}$ \\
\hline UK tariffs & 0.84 & $0.89^{\dagger}$ \\
\hline Visual analogue scale & 84.7 & $87.4^{+}$ \\
\hline
\end{tabular}

N.a.: not available. 'Includes 17 respondents aged 18-20. "Includes: general vocational school, preuniversity school and secondary vocational education ${ }^{* *}$ Includes: professional and academic higher education. ${ }^{\dagger}$ Based on Bernert et al. $(2009)^{19}{ }^{+\dagger}$ Based on Statistics Netherlands $(2013)^{20}$. 


\section{Health state valuation}

We started with an initial model with a random intercept to capture inter-individual differences plus an ARMA model for the unexplained intra-individual response variation over trials, treating all three attributes as categorical variables to allow any kind of relationship between independent and dependent variables, using "one seizure per month", "no to mild side-effects" and "type I seizure" as reference categories for, respectively, "seizure frequency", "experience of side-effects" and "seizure severity", a dummy indicator for every other category of each attribute, and one extra dummy indicator for absence of seizures (to distinguish between absence of seizures and the mildest epilepsy health state, i.e. a type 1 seizure once a month).

Then we compared the model fit with that of a simplified model in which all attributes were included in the model as covariates, thus assuming a linear effect of each attribute on the health state evaluation. This resulted in a significantly worse model fit (Likelihood Ratio test: $\chi^{2}=53.823, d f=7, p<0.005$ ). However, treating only the attribute "experience of side-effects" as a covariate gave a model fit comparable with that of the initial categorical model $\left(\chi^{2}=0.054, d f=1, p>0.05\right)$. Next, we allowed the effect of the covariate "experience of side-effects" to vary randomly between participants, and this random slope was allowed to covary with the random intercept. This resulted in a significantly better model fit $\left(x^{2}=46.039, d f=1, p<0.005\right)$. Finally, we included interactions between all attributes and then reduced the model by deleting nonsignificant interactions. This resulted in a model with an interaction between the attributes "seizure frequency" and "seizure severity", which fitted better than the model without interaction $\left(\chi^{2}=65.213, d f=16, p<0.005\right)$, but at the cost of sixteen additional parameters $(4 * 4)$. Compared to the plotted mean observed utility values (Figure 2.2a), the plotted fixed predicted values of this model (Figure 2.2b) look very similar. To investigate whether the simplified model without interaction is also acceptable, although strictly speaking significantly worse (which may be due to the large sample size), the fixed predicted values of the model without interaction are plotted in Figure 2.2c. This shows that this model is also quite acceptable.

Although Figure 2.2 suggests that the model can be even further simplified by treating the attributes "seizure frequency" and "seizure severity" as continuous covariates and thus assuming their effects to be linear, this model was significantly worse than the model behind Figure $2.2 \mathrm{c}\left(\chi^{2}=55.87, \mathrm{df}=6, \mathrm{p}<0.005\right)$. In relation to the above, it is clinically more relevant to address the attribute "seizure severity" as a categorical rather than continuous predictor, because the successive seizure severity types do not look equidistant clinically ${ }^{2}$, and the attribute "seizure frequency" was not measured on an equidistant scale.

Finally, all other covariates were put into the model (there was no collinearity, i.e. all $\mathrm{VIF}<10)$. Participants' gender, religious belief, educational level, experience with epilepsy and current QoL (EQ-5D and VAS) did not impact the valuation exercise significantly (all $p>0.10$ ). Age was the only characteristic which significantly influenced the health state valuation. The older the participant, the more negative the valuation 
of the health states. This age effect was, however, very small (i.e. 0.001 per year) and the regression coefficients of the attributes hardly changed when age was added to the model. The final model is, therefore, a model with a random intercept, in which "seizure frequency", "seizure severity" and "seizure-free" are categorical predictors, "experience of side-effects" is a continuous predictor with a random slope, and in which no interactions or other covariates are included (Table 2.3).

A
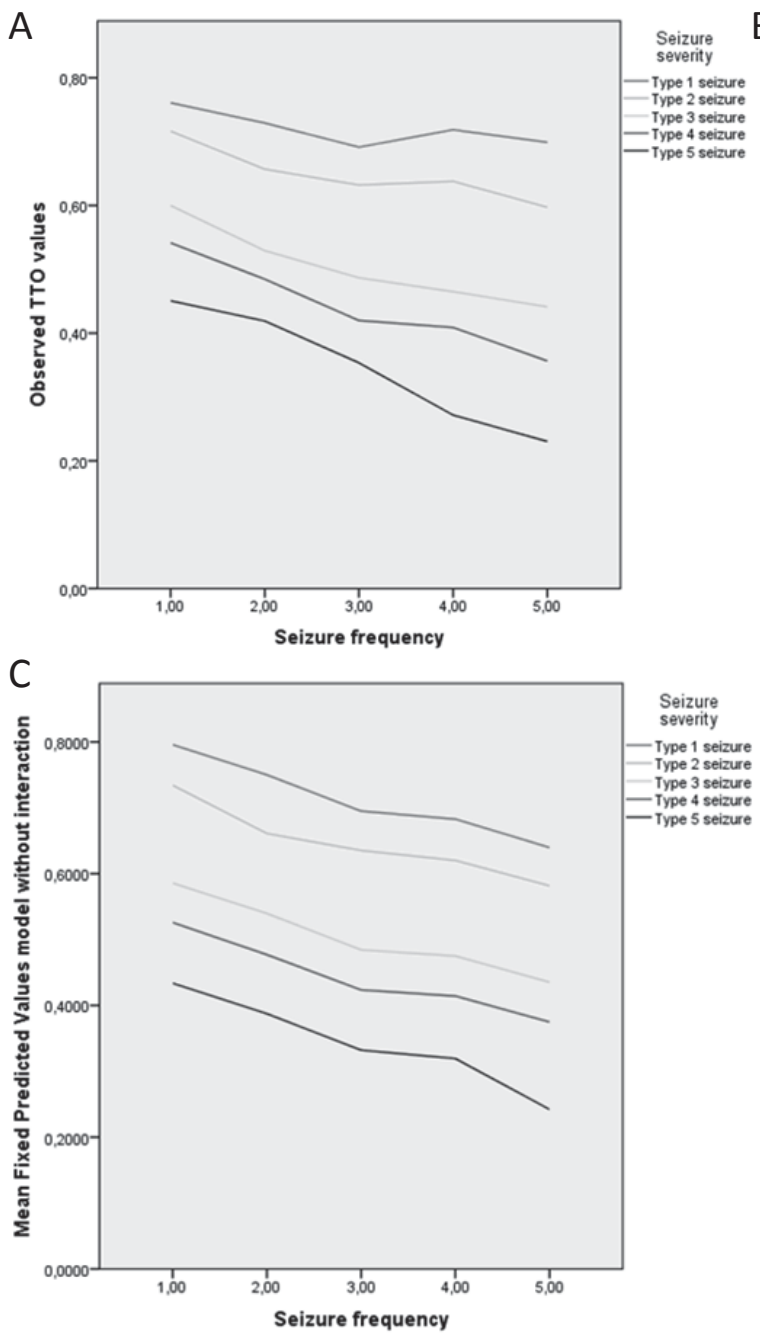

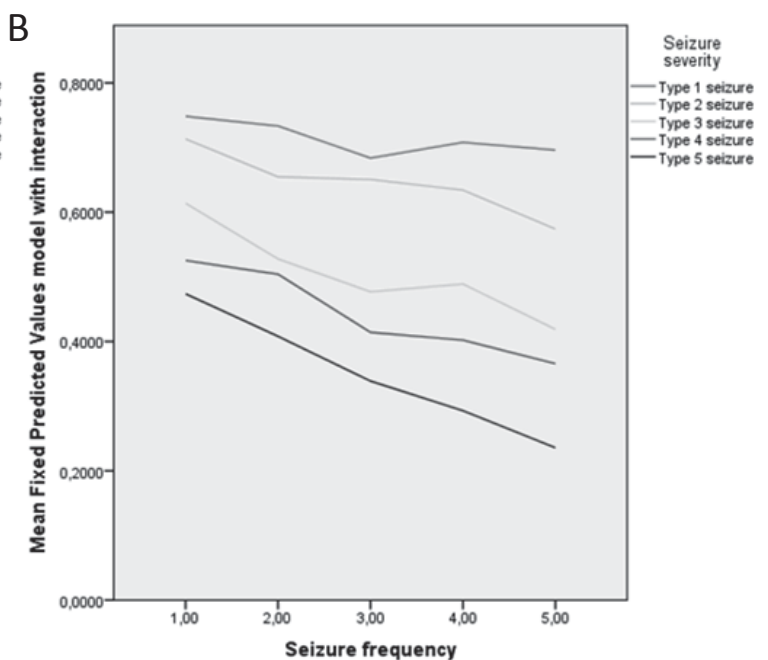

Figure 2.2 Plots of observed values compared to predicted values based on a model with and without interaction terms.

A. Mean observed TTO values per level of seizure frequency, sorted by seizure type. B. Mean predicted values of a model with interaction term between "Seizure frequency" and "Seizure severity"; values per level of seizure frequency, sorted by seizure type. C. Mean predicted values of a model without interaction term; values per level of seizure frequency, sorted by seizure type. Note: Plots are based on a model including the attributes "seizure frequency", "seizure severity", and "experience of side-effects", leaving out the observed and predicted values from the seizure-free condition in these plots. 
Table 2.3 Results from multilevel analysis illustrating influence of variables on health state valuation in the final model ( $t$ between 0 to 10) and for the additional model (SWD, $t$ between -5 to 10).

\begin{tabular}{|c|c|c|c|c|c|c|}
\hline \multirow[b]{2}{*}{ Variable } & \multicolumn{3}{|l|}{ Final model } & \multicolumn{3}{|c|}{ Additional model } \\
\hline & Coefficient & SE & Sign. & Coefficient & SE & Sign. \\
\hline Intercept & .857 & .023 & .000 & .861 & .013 & .000 \\
\hline \multicolumn{7}{|l|}{ Seizure frequency } \\
\hline \multicolumn{7}{|l|}{ Once a month $^{\dagger}$} \\
\hline Once a week & -.045 & .009 & .000 & -.053 & .010 & .000 \\
\hline Twice a week & -.102 & .009 & .000 & -.110 & .010 & .000 \\
\hline Once per day & -.113 & .009 & .000 & -.130 & .010 & .000 \\
\hline Twice per day & -.150 & .008 & .000 & -.171 & .009 & .000 \\
\hline Experience of side-effects ${ }^{*}$ & -.061 & .004 & .000 & -.068 & .004 & .000 \\
\hline \multicolumn{7}{|l|}{ Seizure severity description } \\
\hline \multicolumn{7}{|l|}{ Seizure type $1^{+}$} \\
\hline Seizure type 2 & -.060 & .009 & .000 & -.058 & .010 & .000 \\
\hline Seizure type 3 & -.208 & .009 & .000 & -.216 & .010 & .000 \\
\hline Seizure type 4 & -.273 & .009 & .000 & -.290 & .010 & .000 \\
\hline Seizure type 5 & -.361 & .008 & .000 & -.391 & .009 & .000 \\
\hline Seizure-free ${ }^{* *}$ & .037 & .011 & .001 & .028 & .013 & .024 \\
\hline
\end{tabular}

SWD: states worse than death; SE: standard error; Sign.: significance. ${ }^{*}$ Experience of side-effects is coded as 0 , no to mild side-effects; 1 , minor side-effects; 2 , severe side-effects. ${ }^{* *}$ Seizure-free is coded as 0 , no; 1 , yes

Note: Both models contain no interactions or other covariates; the random part consists of a random intercept plus random slope of the attribute "experience of side-effects" (with intercept-slope covariance) for the between-subject part, and ARMA11 for the within-subject part, of the covariance pattern; the results are based on REML estimation. ${ }^{\dagger}$ Reference category.

All attributes significantly affected patients' health state valuation $(p<0.05)$. Furthermore, the coefficient of the indicator "seizure-free" represents the increase in health state value of being seizure-free as compared to having a type 1 seizure once a month (i.e. reference category), keeping side-effects the same.

Finally, the constant term can be interpreted as the average utility for a patient with one seizure per month of seizure type 1 and who experiences no to mild side-effects. The utility-function, including example utility calculations for three hypothetical health states, can be found in Figure 2.3. Furthermore, when looking at the total range of impact of each attribute (i.e. difference between "best" level and "worst" level), these results indicate that "seizure type" has a substantially larger maximum effect on health state valuation than "seizure frequency" and "experience of sideeffects", and that the latter two attributes have almost the same maximum effect (bearing in mind the coding of experience of side-effects).

\section{Additional analyses}

Table 2.3 also includes the results from the random effects model illustrating the influence of variables on health state valuation when allowing participants to value SWD. The inclusion of SWD results in a slightly higher constant term, and all regression coefficients become larger in absolute terms except for the coefficient, 
seizure-free. This seems logical as the limit of valuation of a health state in the lead TTO was not 0 , but -0.5 , as lower boundary.

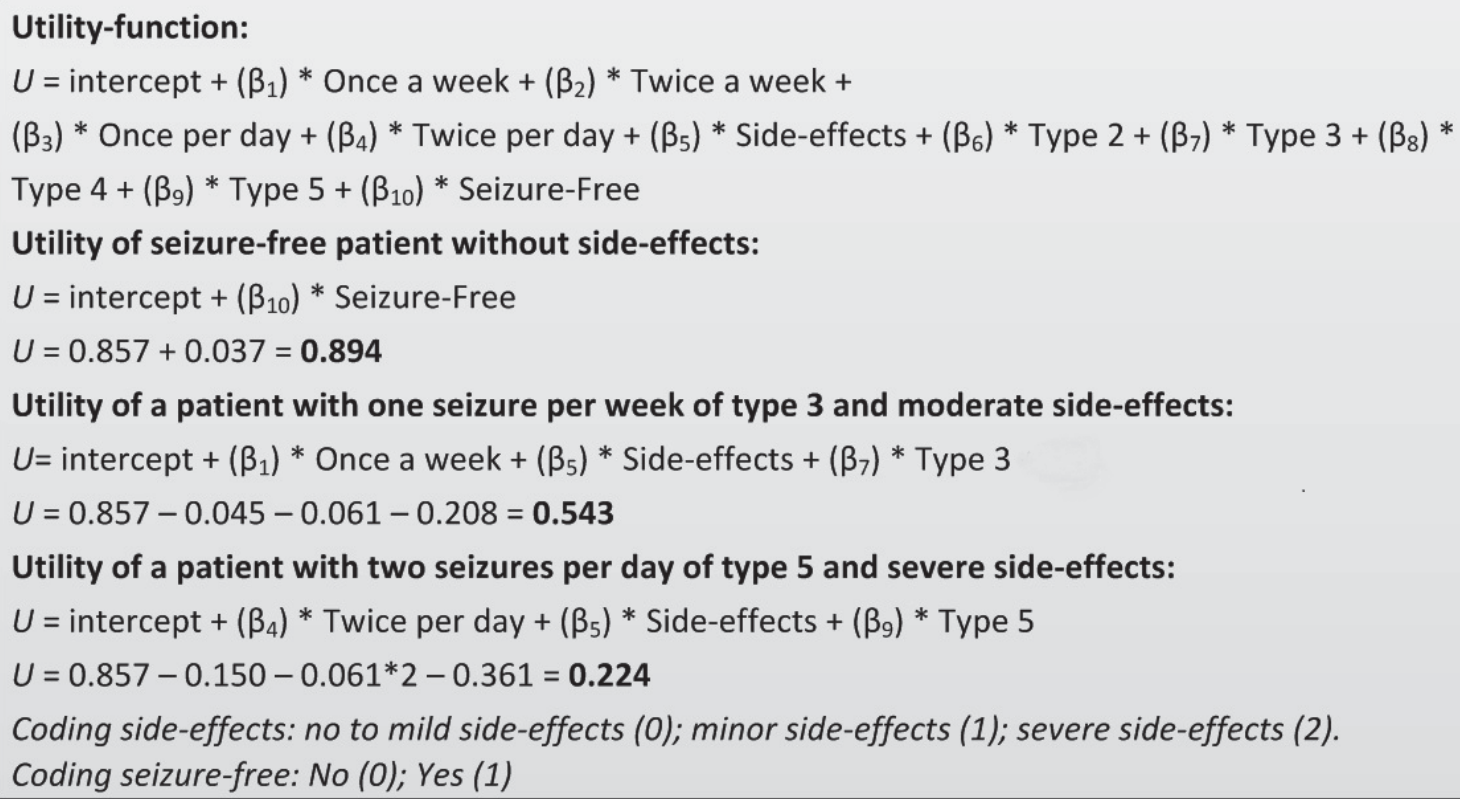

Figure 2.3 Utility-function and examples of utility calculations of three different health states.

\section{Conclusions}

This study has provided utility values for 78 health states based on three clinically important epilepsy attributes (e.g. seizure frequency, seizure severity, side-effects), expressed as a utility-function, which may well prove of value in future (model-based) economic analyses involving epilepsy. The results suggest a utility-function that could be used when health states, described by seizure frequency, seizure severity and sideeffects are available to predict a utility score for any health state described by these variables. In short, our study shows that seizure frequency, seizure severity and sideeffects have a significant impact on QoL. However, looking at the results more closely, this study clearly demonstrates that seizure severity has a greater negative impact on utility compared with the attributes seizure frequency or side-effects.

It is important that utility values are accurately assessed, because in the absence of accurate values, researchers performing cost-effectiveness analyses are often forced to guess the utility values for disease states, possibly leading to extreme variability and questionable results. This study provides a utility-function to transform multiple epilepsy health states into utilities valued by a fairly large sample of the general Dutch population and elicited using established methods. As a result this utility-function is suitable for economic evaluations. 
This study has some limitations that deserve consideration. Firstly, our sample was a convenience sample, not fully representative of the general Dutch population. The sample consisted of slightly more women, was relatively young, quite highly educated, and their own quality of life was somewhat lower than that of the general Dutch population. Secondly, the TTO exercise was conducted via a computerized outward titration method. Although participants could consult the researcher via email or telephone in case of questions/difficulties, there was no interviewer present during the computerized exercise. Most participants (88.2\%) indicated that they had understood the exercise; however, only $46.1 \%$ said the questions were easy. Participants indicated that the health descriptions were clear (87.3\%) but that it was sometimes difficult to make a choice between the health states $(43.8 \%)$, or to become indifferent between the described epilepsy health state and a shorter health state in perfect health (45.9\%). Most of the participants (88\%) needed less than 30 minutes to complete the TTO exercise; only a small number of patients ( $0.6 \%)$ needed 1.5 hours. Finally, although most participants indicated that they had understood the exercise, potential misunderstanding or fatigue can lead to biased results.

Large differences are found between studies presenting utility values for epilepsy health states. ${ }^{4-7}$ This is due to differences in health state descriptions, the sample used to value health states (e.g. patients or general population), and the elicitation method used. ${ }^{13}$ The latter issue, is for example also present in our study, although not surprisingly, the coefficients measured with the 'normal' TTO differed from those measured with the 'Lead-time' TTO.

Obviously, as utilities are used to calculate QALYs necessary for economic evaluations, which compare costs and benefits of alternative interventions to see whether the new intervention offers good 'value for money', they need to be measured in a methodologically sound and preferably uniform manner. The recommendation outlined by the ISPOR RCT-CEA Task force regarding the measurement of utilities, called for the use of generic preference-based outcome instruments, such as the EuroQol (EQ-5D), or for direct utility elicitation methods, such as the standard gamble or time trade-off exercises. ${ }^{14}$ Despite the growing use of generic preference-based outcome instruments such as the EQ-5D, there are many clinical studies, which have recorded outcome information only in the form of non-preference-based instruments. Likewise, researchers working in the field of epilepsy are primarily concerned with measuring symptoms, disease progression and disease-specific QoL and the instruments to do this are generally referred to by economists as non-preferencebased measures. On the one hand, using clinically relevant epilepsy outcome measures to calculate utilities, as is done in this study, may have the merit of sensitivity to disease-specific effects of interventions. On the other hand, the loss of comparability of utilities will fully overrule this gain.

As long as there is no overall consensus on the use of utilities and how to measure them, it will remain a real challenge to deal with this issue properly. Therefore, the use of utilities derived from clinically relevant outcome measures for cost-utility 
analyses is warranted only under strict conditions. Because of the difference in scope of both methods, utility values derived from clinical outcome measures are not comparable with those derived from a generic preference-based QoL instrument, even though they appear to lie on the same scale (0 to 1 ). The development of a utility-function bridging the gap between clinically relevant outcome measures and utilities is valuable for research purposes, although there is concern about the application of our results in economic evaluations. First of all, when health states in a preference elicitation study solely consist of clinical outcome measures, rather than general items of QoL, focusing effects may occur. ${ }^{15,16}$ The context of the valuation exercise is namely more specific, and therefore an exaggeration of health problems may occur, which will be reflected by lower utilities. Secondly, and in contrast to the previous statement, an upward bias of utility scores may occur, as the focus of clinical measures is solely on the physiological aspects of the disease itself, therefore, capturing co-morbidities and/or psychological aspects of the disease might be difficult. ${ }^{15,16}$ Thirdly, developing a utility-function from clinical outcome measures to utilities is not a clear-cut exercise. Decisions made may vary per study and will, therefore, hamper comparability between studies. ${ }^{17,18}$

To conclude, this study provides a utility-function for epilepsy health states and gives insight into the relative impact of clinical outcomes in epilepsy on QoL. The possible increase in sensitivity of outcome measures by using our utility-function comes at the price of a loss of comparability between utilities. We, therefore, advise only using these utility values (or others) in economic evaluations when quality of life is not directly measured in a study and hence, no health state utilities are available, or when there is convincing empirical evidence of the insensitivity of a generic QoL-instrument within the field of epilepsy. Furthermore, when doing so, explicitly state the imposed bias, as using these disease-specific utilities may come at the cost of comparability of utility values within and across conditions. Finally, it is worth stating that all forms of translation-methods are always 'second best', and the existence of these techniques should not be taken as an argument to rely on them instead of obtaining utilities via generic preference-based QoL measurements in prospectively designed studies. 


\section{References}

1. Fisher RS, van Emde Boas W, Blume W, Elger C, Genton P, Lee P, Engel J Jr. Epileptic seizures and epilepsy: definitions proposed by the International League Against Epilepsy (ILAE) and the International Bureau for Epilepsy (IBE). Epilepsia. 2005;46:470-2.

2. O'Donoghue MF, Duncan JS, Sander JW. The National Hospital Seizure Severity Scale: a further development of the Chalfont Seizure Severity Scale. Epilepsia. 1996;37:563-71.

3. Drummond MF, Sculpher MJ, Torrance GW, et al. Methods for the economic evaluation of health care programmes (3rd ed.). Oxford: Oxford University Press, 2005.

4. Messori A, Trippoli S, Becagli P, Cincotta M, Labbate MG, Zaccara G. Adjunctive lamotrigine therapy in patients with refractory seizures: a lifetime cost-utility analysis. Eur J Clin Pharmacol. 1998;53:421-7.

5. Forbes RB, Macdonald S, Eljamel S, Roberts RC. Cost-utility analysis of vagus nerve stimulators for adults with medically refractory epilepsy. Seizure. 2003;12:249-56.

6. Carroll AE, Downs SM. Improving decision analyses: parent preferences (utility values) for pediatric health outcomes. J Pediatr. 2009;155:21-5,25 e1-5.

7. Kang HJ, Kang E, Jo MW, Park EJ, Yoon S, Lee EK. The utility score of epilepsy with partial seizure measured by TTO, VAS, and EQ-5D in the general Korean population. Epilepsy Res. 2014;108:963-71.

8. Lamers LM, McDonnell J, Stalmeier PF, Krabbe PF, Busschbach JJ. The Dutch tariff: results and arguments for an effective design for national EQ-5D valuation studies. Health Econ. 2006;15: 1121-32.

9. EuroQol G. EuroQol--a new facility for the measurement of health-related quality of life. Health Policy. 1990;16:199-208.

10. Verbeke G, Molenberghs G. Linear mixed models for longitudinal data. New York: Springer, 2000.

11. Robinson A, Spencer A. Exploring challenges to TTO utilities: valuing states worse than dead. Health Econ. 2006;15:393-402.

12. Dolan $P$, Gudex $C$, Kind $P$, Williams A. The time trade-off method: results from a general population study. Health Econ. 1996;5:141-54.

13. Dolan P. The measurement of health-related quality of life. In: Culyer AJ, Newhouse JP, eds., Handbook of Health Economics. The Netherlands: North-Holland, 2000.

14. Ramsey S, Willke R, Briggs A, Brown R, Buxton M, Chawla A, Cook J, Glick H, Liljas B, Petitti D, Reed S. Good research practices for cost-effectiveness analysis alongside clinical trials: the ISPOR RCT-CEA Task Force report. Value Health. 2005;8:521-33.

15. Brazier J, Tsuchiya A. Preference-based condition-specific measures of health: what happens to cross programme comparability? Health Econ. 2010;19:125-9.

16. Fryback DG, Lawrence WF, Jr. Dollars may not buy as many QALYs as we think: a problem with defining quality-of-life adjustments. Med Decis Making. 1997;17:276-84.

17. Arnesen T, Trommald M. Are QALYs based on time trade-off comparable?-A systematic review of TTO methodologies. Health Econ. 2005;14:39-53.

18. Lenert LA, Cher DJ, Goldstein MK, Bergen MR, Garber A. The effect of search procedures on utility elicitations. Med Decis Making . 1998;18:76-83.

19. Bernert S, Fernández A, Haro JM, König HH, Alonso J, Vilagut G, Sevilla-Dedieu C, de Graaf R, Matschinger $\mathrm{H}$, Heider D, Angermeyer MC; ESEMeD/MHEDEA 2000 Investigators. Comparison of different valuation methods for population health status measured by the EQ-5D in three European countries. Value Health. 2009;12:750-8.

20. CBS. Population numbers. The Hague/Heerlen: Statistics Netherlands, 2013. 


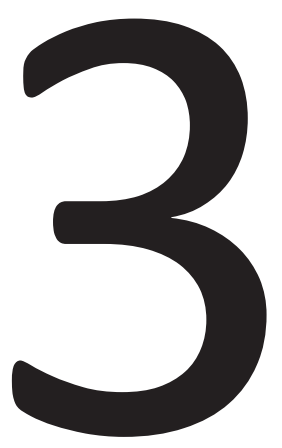

\section{Eliciting patients' preferences for epilepsy diagnostics: A discrete choice experiment}

BFM Wijnen, RJA de Kinderen, AJ Colon, CD Dirksen, BAB Essers, M Hiligsmann, FSS Leijten, PPW Ossenblok, SMAA Evers

Epilepsy and Behavior 2014;31:102-109 


\section{Abstract}

\section{Background}

Diagnosing epilepsy is a lengthy and burdensome process for patients and their family. Although the need for a more patient-centered approach in clinical practice is widely acknowledged, empirical evidence regarding patient preferences for diagnostic modalities in epilepsy is missing. The objectives of this study were 1) to identify to what extent important attributes of diagnostic procedures in epilepsy affect preferences for a procedure, 2) to determine the relative importance of these attributes, and 3) to calculate overall utility scores for routine electroencephalography (EEG) and magnetoencephalography (MEG) recordings.

\section{Methods}

A discrete choice experiment was performed to determine patients' preferences, which involved presentation of pairwise choice tasks regarding hypothetical scenarios. Scenarios varied along six attributes: "way of measuring brain activity", "duration", "freedom of movement", "travel time", "type of additional examination", and "chance of additional examination". Choice tasks were constructed using a statistically efficient design, and the questionnaire contained 15 unique unlabeled choice tasks. Mixed multinomial logistic regression was used to estimate patients' preferences.

\section{Results}

A total of 289 questionnaires were included in the analysis. McFadden's pseudo $R^{2}$ showed a model fit of 0.28 , and all attributes were statistically significant. Heterogeneity in preferences was present for all attributes. "Freedom of movement" and "Chance of additional examination" were perceived as the most important attributes. Overall utility scores did not substantially differ between routine EEG and MEG.

\section{Conclusion}

This study suggests that the identified attributes are important in determining patients' preference for epilepsy diagnostics. It can be concluded that MEG is not necessarily more patient-friendly than a routine EEG in primary diagnostics and, regarding additional diagnostics, patients have a strong preference for long-term 24-h EEG over EEG after sleep deprivation. Furthermore, barring substantial heterogeneity within the parameters in mind, our study suggests that it is important to take individual preferences into account in medical decisionmaking. 


\section{Introduction}

Epilepsy is a severe brain disease with a large impact on patients' quality of life ${ }^{1,2}$, their family and surroundings, and society as a whole. ${ }^{3,4}$ It is one of the most prevalent noncommunicable neurologic diseases and estimated to affect almost 70 million people around the world. ${ }^{5,6}$ Internationally, the incidence of epilepsy is estimated at 50 per 100,000 people, with large differences between high-income countries $(45 / 100,000)$ and low- and middle-income countries $(82 / 100,000) .{ }^{5-7}$ Diagnosing epilepsy is a lengthy process that can be a burden for the patient and has a large impact on the health system, while misdiagnoses are quite common. Of patients diagnosed with epilepsy who then are evaluated at epilepsy centers, $20 \%$ to $30 \%$ are found to have been misdiagnosed..$^{8-10}$

According to several European guidelines, ${ }^{11-13}$ it is recommended that an electroencephalography (EEG) should be performed to support a diagnosis of epilepsy in patients in whom the clinical history suggests that the symptoms are likely to be epileptic in origin. Consequently, EEG is the most used diagnostic procedure for epilepsy. ${ }^{14}$ However, its sensitivity and specificity depend on several factors such as age and recording procedures (e.g., activation procedures such as provoked hyperventilation or photic stimulation). ${ }^{14} \mathrm{~A}$ routine EEG recording in a patient with epilepsy will show no epileptiform activity in about $50 \%$ of cases. ${ }^{15}$ Hence, additional EEG examinations are needed to increase diagnostic yield by increasing epileptiform activity without inducing seizures. To this extent, EEG after sleep deprivation (EEGsd) and long-term 24-h EEG are generally recommended. ${ }^{11-13,15}$ The latter is often used in children or patients with mental illness, since an EEGsd is considered to be too burdensome for these patients.

Nonetheless, both 24-h EEG and EEGsd have considerable disadvantages. Sleep deprivation is shown to elicit seizures, ${ }^{15-18}$ which is supported by the fact that it is still one of the three most important seizure-inducing factors mentioned by patients in questionnaires. $^{19-21}$ The 24-h EEG impairs patients in their daily routine; wires attached to the portable EEG recorder constrain them from physical exercises and limit their freedom of movement.

An alternative to these diagnostic procedures is magnetoencephalography (MEG), which seems to be a more patient-friendly way to detect epileptiform discharges, ${ }^{22}$ potentially with great diagnostic value in the early stage of the diagnostic process. ${ }^{22-24}$ Magnetoencephalography is a noninvasive technique that enables recording of epileptiform discharges over the whole head, with minimal preparations. Nevertheless, MEG also has some considerable disadvantages; most importantly, a MEG recording requires that patients move as little as possible, which could be very limiting for some patients.

It is important to include patients' preferences in evaluation of health technologies as patients differ from each other in their biologic, social, or cultural characteristics as well as in their preferences. Although the need for a more patient-centered approach 
in clinical practice and evaluation studies is widely acknowledged ${ }^{25-27}$ and patients' preferences have been shown to significantly impact their willingness to use health care services, ${ }^{28-30}$ empirical evidence is missing regarding patients' preferences for routine EEG, EEGsd, 24-h EEG, and MEG as diagnostic tools in epilepsy. Using a discrete choice experiment (DCE), the present study attempted to determine patients' preferences for different elements of the diagnostic trajectory of epilepsy. The DCE involved presentation of a series of pairwise choice tasks regarding hypothetical scenarios. ${ }^{31,32}$ Discrete choice experiments have increasingly been used in health care as an approach to elicit patients' preferences for medication attributes and have been applied in similar cases (e.g. patients' preferences regarding several screening tests). ${ }^{33-37}$

Therefore, the objectives of this study were 1) to identify to what extent important attributes of diagnostic procedures in epilepsy affect patients' preferences for a procedure, 2) to determine the relative importance of these attributes, and 3) to calculate overall utility scores for routine EEG and MEG recordings.

\section{Methods}

\section{Discrete choice experiment}

A DCE is an attribute-based stated preference valuation technique. ${ }^{32}$ In DCEs, individuals are presented with a series of hypothetical choice tasks and are asked to express a preference. The technique is based on the premises that health care interventions can be described by a number of attributes and that preferences for these interventions are influenced by the variations (i.e., levels) within these attributes (see Table 3.1). ${ }^{32}$

Using these attributes and levels, one can construct a "scenario" by choosing a level for each attribute. Subsequently, respondents are asked to indicate, by pairwise comparison of alternative scenarios, which would be their preferred option (see Figure 3.1). In this way, respondents are encouraged to make a trade-off between attributes and their corresponding levels. Afterwards, the relative importance of each attribute (and corresponding levels) can be identified using a regression model.

In this study, an unlabeled DCE is used (i.e. the alternatives are named "Method A" and "Method B", instead of, e.g. EEG and MEG) mainly because the labeled alternatives themselves convey information to respondents. Thus, in a labeled design, respondents may use labeled alternatives to deduce information which they perceive as missing, particularly when patients are familiar with some of the alternatives. ${ }^{38}$ Hence, an unlabeled DCE might encourage respondents to choose an alternative by only trading-off attribute levels. ${ }^{38}$ 
Discrete choice experiment methodology is well described in the literature. ${ }^{31,32}$ This study was conducted according to the stepped approach proposed by Ryan and Farrar. $^{39}$

Table 3.1 Attributes and levels for epilepsy diagnostics.

\begin{tabular}{ll}
\hline Attributes & Levels \\
\hline Way of measuring brain activity & Sensor net (Base case*) \\
& Helmet \\
& Electro cap \\
& 30 minutes \\
Duration of recording** & 60 minutes \\
& 90 minutes \\
Freedom of movement & Minor movements possible (Base case*) \\
& No movements possible \\
Travel time & 30 minutes \\
& 60 minutes \\
& 90 minutes \\
Type of additional examination & $24 \mathrm{~h}$-EEG (Base level*) \\
& EEG after sleep deprivation \\
Chance of additional examination** & 20 out of 100 patients \\
& 35 out of 100 patients \\
& 50 out of 100 patients \\
\hline
\end{tabular}

* Level of the attribute which was used as the reference level. ** Estimated as continuous variable within the MMNL model.

\begin{tabular}{lll}
\hline Characteristics & Method A & Method B \\
\hline Way of measuring brain activity & By use of a helmet & By use of an electro cap \\
Duration of registration & 30 minutes & 90 minutes \\
Freedom of movement & No movements possible & Minor movements possible \\
Travel time & 30 minutes & 90 minutes \\
Type of additional examination & EEG after sleep deprivation & 24-hour EEG \\
Chance of additional examination & $50 \%$ (50 out of 100 patients) & 20\% (20 out of 100 patients) \\
\hline Which option do you prefer? & $\square$ & $\square$ \\
\hline
\end{tabular}

Figure 3.1 Example of unlabeled choice set as presented to patients.

\section{Identifying attributes and levels}

Attributes were identified based on literature review, ${ }^{11,12,14,15,22}$ expert and patient interviews ( $\mathrm{N}=7$; three neurologists, two patients, and two laboratory technicians), and observations during clinical practice. Expert and patient interviews were semistructured to give participants the opportunity to explain their thoughts rather than forcing them to choose from fixed responses. In our case, because of the impact on the random component variability ${ }^{40}$ and to minimize cognitive burden ${ }^{41}$, the number of attributes was limited to six. Other attributes were considered to be less relevant or were left out because of commonality between attributes. The final attributes, based on a consensus meeting with clinicians and experts, were 1 ) way of 
measuring brain activity, 2) duration of the recording (DURATION), 3) freedom of movement (FOM), 4) travel time (TT), 5) type of additional examination (TAE), and 6) chance of additional examination (CAE) (see Table 3.1). Levels for the first attribute were chosen to represent a routine EEG recording (electro cap), MEG recording (helmet), and routine EEG recording with a HydroCel Geodesic Sensor Net. ${ }^{42}$ The addition of the sensor net, which is said to not require any scalp preparation or abrasion, was merely to see what the impact of future improvements in EEG recording might offer to patients.

Levels for the attribute "chance of additional examination" were based on previous studies regarding the sensitivity of MEG and EEG. ${ }^{14,22,23,43,44}$ Other levels were determined based on expert opinion (i.e. travel time and type of additional examination) and clinical characteristics of each recording (i.e. duration of recording and freedom of movement). Travel time was taken into account as up to now the availability of MEG in the Netherlands is limited.

\section{Designing choice sets and questionnaire}

The choice sets are constructed using Ngene (version 1.1.1, http:// www.choicemetrics.com/). Four attributes with three levels and two attributes with two levels result in $3^{4} * 2^{2}=324$ hypothetical scenarios. Hence, for practical reasons, a fractional factorial design (subset of hypothetical scenarios) was used.

The fractional factorial design was constructed using Bayesian principles, a so-called Bayesian optimal or statistically efficient design. Using an efficient design, it is possible to maximize the precision of estimated parameters for a given number of choice tasks (by maximizing D-efficiency). ${ }^{45,46}$ The statistically efficient design approach assumes a prior distribution of likely parameter values (e.g. the beta coefficients in the regression analysis) for some or all parameters. ${ }^{47}$ For example, it is reasonable to assume that patients prefer a shorter duration of recording and travel time. Therefore, these attributes were assumed to have a negative sign (i.e. longer duration decreases patients' preferences).

Within each choice task, respondents were asked to choose between two unlabeled scenarios (see Figure 3.1). We did not include an opt-out option representing the alternative 'no diagnostic testing'. In this way, respondents were forced to make trade-offs between attributes, and it ensured that respondents could not avoid difficult decisions. ${ }^{48,49}$ Moreover, little information is captured in terms of attribute level trade-offs when such an option is selected.

After a short introduction to the study, respondents were given a detailed description about the DCE task. Next, attributes and levels were explained, and an example of a choice set was shown. Subsequently, the choice tasks were presented and, lastly, respondent characteristics were collected.

The final version of the questionnaire contained 15 unique choice tasks. To evaluate the test-retest reliability of the questionnaire, one choice task was repeated at the 
end of the questionnaire. This makes a total of 16 choice tasks per respondents, which is considered good practice and cognitively acceptable. ${ }^{50,51}$ Afterwards, respondents were asked to evaluate the difficulty of the tasks on a seven-point scale ranging from "very easy" to "very difficult". The questionnaire was pilot-tested among experts to ensure that the levels of each choice were relevant and comprehensible. The full questionnaire in Dutch is available from the authors on request.

\section{Data collection and participants}

The study was conducted in the outpatient clinic of Kempenhaeghe, a center noted for its expertise in the field of epilepsy, and at the University Medical Centre Utrecht (UMCU). Both involved parties have a leading role in the application of MEG in clinical epilepsy research.

Data were collected between February and April 2013. The DCE questionnaire was sent by mail to 1009 patients, completed by the patients at home, and returned in a postage-paid envelope. All patients had to be at least 18 years of age, diagnosed as having epilepsy, visited the outpatient clinic of Kempenhaeghe in the past year, and home dwelling. Patients living in specialized health care institutions (i.e. for patients with mental handicaps) were excluded from the study. Because of the limited application of MEG in primary diagnostics of epilepsy, we deliberately contacted patients from UMCU who are experienced with MEG to ensure that these patients were represented in the sample.

Only patients who agreed to be contacted for participation in (clinical) studies were approached. An information letter attached to the questionnaire was sent to these patients, which explained that by returning the questionnaire, respondents consented to participate in the study. Approval for this consent procedure and study was obtained from the ethics committee of Kempenhaeghe and UMCU.

\section{Statistics and data analysis}

Discrete choice data were analyzed using Nlogit version 5 (Econometric Software, Inc.). A mixed multinomial logit (MMNL) model was used to determine the effect of the attribute levels on patients' preferences. Respondents who completed less than five choice sets were excluded from the analysis.

Mixed multinomial logit was chosen to allow for possible preference heterogeneity across respondents and to account for the panel nature of the data. ${ }^{38,52} \mathrm{~A} \mathrm{MMNL}$ model is constructed regarding the assumption that parameters are randomly distributed in the population. Heterogeneity within these parameters can be assessed by estimating the standard deviation of the parameters. ${ }^{46}$ Therefore, MMNL is able to relax some or all of the assumptions of the standard multinomial logit model, most importantly homogeneity of patients' preferences. ${ }^{52}$ 
The six attributes in the DCE were modeled as determinants of whether respondents chose "Method A" or "Method B", hence the difference in utility between both scenarios. Therefore, the regression function to be estimated had the following form:

$$
\begin{aligned}
V_{\mathrm{ij}}= & \left(\beta_{1}+\eta_{1 \mathrm{i}}\right) * \text { HELMET }+\left(\beta_{2}+\eta_{2 \mathrm{i}}\right) * \text { ELECTROCAP } \\
& +\left(\beta_{3}+\eta_{3 \mathrm{i}}\right) * \text { DURATION }+\left(\beta_{4}+\eta_{4 \mathrm{i}}\right) * \text { FOM }+\left(\beta_{5}+\eta_{5 \mathrm{i}}\right) * \text { TT } \\
& +\left(\beta_{6}+\eta_{6 \mathrm{i}}\right) * \text { TAE }+\left(\beta_{7}+\eta_{7 \mathrm{i}}\right) * \text { CAE }+\varepsilon_{\mathrm{ij}}
\end{aligned}
$$

in which the value of the regression coefficients indicate the relative importance of the attribute, $V$ represents the observable relative utility, and $\eta_{i}$ represents the standard deviation of the random parameter. Lastly, $\eta_{i j}+\varepsilon_{i j}$ captures the individualspecific unexplained variance around the mean. ${ }^{46}$ No constant term was included in the final model as the constant term represents the mean of the unobserved effects for each of the alternatives, after accounting for the role each attribute plays in the decision-making process. ${ }^{52}$ Hence, in an unlabeled design, a significant constant term would be an indication of left-to-right bias (i.e., tendency to consistently choose either the first or the second alternative in the choice task). ${ }^{48}$ To describe categorical variables, dummy coding was used for ease of interpretation (base-case levels can be found in Table 3.1).

We tested interactions between attributes and between attributes and respondent characteristics. All parameters included in the MMNL model were treated as random parameters and estimated using 2000 Halton draws. Parameters were tested for significance at $\alpha=0.05$ and assumed to be normally distributed. Next, model fit was assessed using log likelihood and McFadden's pseudo $\mathrm{R}^{2}$. To test for left-to-right bias, the constant term was included in the initial analyses. To account for scale differences and ease of interpretation, relative importance weights were calculated using the method described by Malhotra and Birks. ${ }^{53}$ For continuous variables, we based this calculation on the total range of the attribute.

Reliability analyses were conducted to explore the impact of respondents who failed the test-retest and to explore the impact of differences in perceived difficulty. Additional analyses were performed on age (two groups, split by mean value), educational level (two groups, split by mean value), and gender by means of creating interaction terms within the final model. These analyses were done to assess the potential impact of covariates and to explain some heterogeneity within the data.

Results of the MMNL model were translated to clinical practice. As mentioned above, levels for each attribute were chosen based on expert opinion and the clinical characteristics of each recording. Using this information, one can (hypothetically) compare routine EEG and MEG by selecting levels which closely resemble the clinical features of each recording and calculate overall utility scores using the utility function derived by the MMNL model. The lower the overall utility score, the less preferred that scenario is. 
Data file and additional analyses can be retrieved by contacting the corresponding author.

\section{Results}

\section{Respondent characteristics}

A total of 304 respondents (30.1\%) completed the DCE questionnaire. Excluding respondents who completed five choice tasks or less, a total of 289 questionnaires were included in the analysis. Mean age of the respondents was 45.5 years. Nearly all respondents reported to have experience with epilepsy diagnosis using EEG techniques; only $5.9 \%$ reported not to have experience with any of the techniques. Other patient characteristics are presented in Table 3.2. No significant differences were found between responders and nonresponders for all respondent characteristics.

Table 3.2 Respondent characteristics.

\begin{tabular}{lll}
\hline Characteristic & & Total cohort (N=289) \\
\hline Gender & Male & $130(45 \%)$ \\
& Female & $158(55 \%)$ \\
Age(y) & Missing & $1(0.3 \%)$ \\
& Mean & 45.5 years \\
& $20-34$ & $80(27.7 \%)$ \\
& $35-49$ & $91(31.5 \%)$ \\
Educational level & $50-64$ & $82(28.4 \%)$ \\
& $65-85$ & $33(11.4 \%)$ \\
& Missing & $3(1.0 \%)$ \\
Intervention experience* & Primary & $83(28.7 \%)$ \\
& Secondary education & $123(42.6 \%)$ \\
& Secondary vocational education & $55(19.0 \%)$ \\
& Higher education & $22(7.6 \%)$ \\
& Missing & $6(2.1 \%)$ \\
& Electro cap & $219(75.8 \%)$ \\
& Helmet & $63(21.8 \%)$ \\
& EEGsd & $152(52.6 \%)$ \\
& 24h-EEG & $164(56.7 \%)$ \\
& No experience & $17(5.9 \%)$ \\
& Missing & $2(0.7 \%)$ \\
& Relatively easy (range: 1 - 3) & $114(39.4 \%)$ \\
& Neutral (range: 4) & $61(21.1 \%)$ \\
& Relatively difficult (range: $5-7)$ & $102(35.3 \%)$ \\
& Missing & $12(4.2 \%)$ \\
\hline
\end{tabular}

* The "sensor net" is not in this table because it is not used in routine practice; thus no respondent could have experience with it. 


\section{DCE choice task}

McFadden's pseudo $\mathrm{R}^{2}$ shows a model fit of 0.28 . All attributes are statistically significant, which provides evidence of the theoretical validity of the model and indicates that the attributes are considered as being important in determining patients' preferences. ${ }^{28}$ The regression coefficient for each attribute level represents the mean part-worth utility of that attribute level in the respondent sample. A negative $\beta$-coefficient indicates that as the level of the attribute increases, utility is decreasing. Results of the MMNL model are presented in Table 3.3.

The estimated coefficients all have the anticipated negative sign, apart from "way of measuring brain activity" and "type of additional examination", of which no specific effect was anticipated.

Table 3.3 Results from mixed multinomial logit model illustrating influence of attributes on utility.

\begin{tabular}{lll}
\hline Attributes & Coefficient $(95 \% \mathrm{Cl})$ & Standard deviation $(95 \% \mathrm{Cl})$ \\
\hline Way of measuring brain activity & & \\
Helmet & $-0.220(-0.445,-0.005)^{* *}$ & $1.243(0.993,1.492)^{* * *}$ \\
Electro cap & $-0.017(-0.203,0.169)$ & $0.896(0.651,1.142)^{* * *}$ \\
Duration (per minute) & $-0.018(-0.022,-0.015)^{* * *}$ & $0.016(0.013,0.020)^{* * *}$ \\
Freedom of movement & $-1.840(-2.117,-1.563)^{* * *}$ & $1.783(1.509,2.056)^{* * *}$ \\
Travel time (per minute) & $-0.015(-0.019,-0.012)^{* * *}$ & $0.020(0.016,0.024)^{* * *}$ \\
Type of additional examination & $-0.939(-1.206,-0.671)^{* * *}$ & $2.001(1.711,2.291)^{* * *}$ \\
Chance of additional examination (\%) & $-0.057(-0.070,-0.045)^{* * *}$ & $0.079(0.067,0.092)^{* * *}$ \\
Log likelihood & -2164.86 & \\
Pseudo R-squared & 0.28 & \\
Number of observations & 4335 & \\
Number of individuals & 289 & \\
\hline
\end{tabular}

$* * *, * * *$ Significance at $1 \%, 5 \%, 10 \%$ level. Table represents $\beta$-coefficients from MMNL model. The regression coefficients represent the mean part-worth utility of that attribute in the respondent sample. $\mathrm{Cl}$ : Confidence interval.

To determine which attribute has the largest impact on patients' preferences, it is convenient to look at the relative importance weights as they represent the importance of each attribute in percentages (see Table 3.4). As shown, "freedom of movement" has the largest impact on patients' preference (27\%). Respondents had a significant preference for scenarios in which minor movements were possible. The attribute with the second largest impact on patients' preference was "chance of additional examination" (26\%), indicating that respondents preferred a lower chance of additional examination. Furthermore, respondents had a significant preference for shorter recording (16\%), shorter travel times (13\%), and significantly preferred $24-\mathrm{h}$ EEG over EEGsd (14\%). As for the way of measuring brain activity (4\%), respondents significantly preferred the sensor net over the helmet but had no significant preference between the sensor net and the electro cap.

Furthermore, standard deviations of the random parameters were significant for all attributes, which is an indication of preference heterogeneity within the sample. 
Hence, this shows the presence of variations in the importance of attributes between respondents. $^{46,52}$

Table 3.4 Relative importance of attributes (in percentage) for the total sample, males and females.

\begin{tabular}{llll}
\hline Attribute & Total sample (N=289) & Males (N=130) & Females (N=159) \\
\hline Way of measuring brain activity & $4 \%$ & $9 \%$ & $2 \%$ \\
Duration & $16 \%$ & $10 \%$ & $17 \%$ \\
Freedom of movement & $27 \%$ & $23 \%$ & $31 \%$ \\
Travel time & $13 \%$ & $21 \%$ & $17 \%$ \\
Type of additional examination & $14 \%$ & $11 \%$ & $16 \%$ \\
Chance of additional examination & $26 \%$ & $26 \%$ & $17 \%$ \\
\hline
\end{tabular}

\section{Differences in preferences between subgroups}

Different subgroup analyses, based on interaction terms within the final model, were examined. Models with interactions between attributes and covariates (educational level, age, experience with MEG/EEG (sd/24 h), difficulty) were tested, but all covariates turned out to be nonsignificant. However, a significant difference was found between males and females. Results of the model for male and female respondents are presented in Table 3.5. Female respondents had a significantly stronger preference for recordings in which minor movements were possible, as can also be seen when looking at the difference in relative importance weights (see Table 3.4). Stratified analysis based on gender had large implications on the relative importance weights. In males, "chance of additional examination" turns out to be the most important attribute (26\%), whereas in females "freedom of movement" is still the most important attribute (31\%). Preference for other attributes did not significantly differ between males and females. Differences between respondents who passed and failed the test-retest are presented in Table 3.6.

\section{Translation to clinical characteristics of diagnostic modalities}

Overall utility scores for routine EEG and MEG were -5.97 and -6.12 (see Figure 3.2). Hence, in general, albeit the difference is marginal, routine EEG is preferred over MEG. However, when stratifying for gender, it can be seen that males prefer MEG over routine EEG, whereas females prefer routine EEG over MEG. As this study mainly focuses on discomforts of each modality, overall utility scores are negative. However, this does not imply that patients would prefer to not be diagnosed with EEG or MEG at all as an opt-out option was not included into the choice tasks. 
Table 3.5 Differences between male and female respondents in their preferences for epilepsy diagnostics.

\begin{tabular}{|c|c|c|c|}
\hline Attributes & Males $(95 \% \mathrm{Cl})$ & Females $(95 \% \mathrm{Cl})$ & P Valuet \\
\hline \multicolumn{4}{|l|}{ Way of measuring brain activity } \\
\hline \multirow[t]{2}{*}{ Helmet } & $-0.36(-0.66,-0.06)^{* *}$ & $-0.09(-0.38,0.20)$ & 0.25 \\
\hline & SD: $1.16^{* * *}$ & SD: $1.30 * * *$ & \\
\hline \multirow[t]{2}{*}{ Electro cap } & $-0.16(-0.43,0.11)$ & $0.05(-0.19,0.29)$ & 0.64 \\
\hline & SD: $0.84^{* * *}$ & SD: $0.74 * * *$ & \\
\hline \multirow[t]{2}{*}{ Duration (per minute) } & $-0.01(-0.02,-0.01) * * *$ & $-0.02(-0.03,-0.02)^{* * *}$ & 0.28 \\
\hline & SD: $0.02 * * *$ & SD: $0.01 * * *$ & \\
\hline \multirow[t]{2}{*}{ Freedom of movement } & $-1.36(-1.74,-0.99) * * *$ & $-2.14(-2.55,-1.73) * * *$ & $0.01 * *$ \\
\hline & SD: $1.65^{* * *}$ & SD: $1.97^{* * *}$ & \\
\hline \multirow[t]{2}{*}{ Travel time (per minute) } & $-0.02(-0.02,-0.01) * * *$ & $-0.02(-0.02,-0.01)^{* * *}$ & 0.71 \\
\hline & SD: $0.02 * * *$ & SD: $0.02 * * *$ & \\
\hline \multirow[t]{2}{*}{ Type of additional examination } & $-0.64(-0.10,-0.27)^{* * *}$ & $-1.12(-1.47,-0.76)^{* * *}$ & 0.25 \\
\hline & SD: $1.96 * * *$ & SD: $1.91 * * *$ & \\
\hline \multirow[t]{2}{*}{ Chance of additional examination (per \%) } & $-0.05(-0.07,-0.04) * * *$ & $-0.04(-0.06,-0.03) * * *$ & $0.08 *$ \\
\hline & SD: $0.09 * * *$ & SD: $0.07^{* * *}$ & \\
\hline Log likelihood & -1029.49 & -1134.19 & \\
\hline Pseudo R-squared & 0.24 & 0.31 & \\
\hline Number of observations & 1965 & 2385 & \\
\hline Number of individuals & 130 & 158 & \\
\hline
\end{tabular}

$* * *, * * *$ Significance at $1 \%, 5 \%, 10 \%$ level; SD: standard deviation; $+p$ Value was estimated in a joint model with interaction terms.

Table 3.6 Differences between respondents who passed the test-retest exercise and respondents who failed the test-retest exercise in their preferences for epilepsy diagnostics.

\begin{tabular}{|c|c|c|c|}
\hline Attributes & $\begin{array}{l}\text { Passed test-retest } \\
\text { exercise }(95 \% \mathrm{Cl})\end{array}$ & $\begin{array}{l}\text { Failed test-retest } \\
\text { exercise }(95 \% \mathrm{Cl})\end{array}$ & P Value ${ }^{\dagger}$ \\
\hline \multicolumn{4}{|l|}{ Way of measuring brain activity } \\
\hline \multirow[t]{2}{*}{ Helmet } & $-0.22(-0.47,0.02)^{*}$ & $-0.25(-0.67,0.18)$ & 0.46 \\
\hline & SD: $1.29 * * *$ & SD: $0.93 * * *$ & \\
\hline \multirow[t]{2}{*}{ Electro cap } & $-0.16(-0.38,0.04)$ & $0.36(0.04,0.67)^{* *}$ & $0.04^{* *}$ \\
\hline & SD: $0.91 * * *$ & SD: 0.04 & \\
\hline \multirow[t]{2}{*}{ Duration (per minute) } & $-0.02(-0.02,-0.02)^{* * *}$ & $-0.01(-0.01,0.00)^{* * *}$ & 0.67 \\
\hline & SD: $0.02 * * *$ & SD: $0.01 * * *$ & \\
\hline \multirow[t]{2}{*}{ Freedom of movement } & $-2.01(-2.35,-1.67)^{* * *}$ & $-1.07(-1.44,-0.7)^{* * *}$ & 0.85 \\
\hline & SD: $1.92 * * *$ & SD: $0.71 * * *$ & \\
\hline \multirow[t]{2}{*}{ Travel time (per minute) } & $-0.02(-0.02,-0.01)^{* * *}$ & $-0.01(-0.01,0.0)^{* * *}$ & 0.89 \\
\hline & SD: $0.02 * * *$ & SD: $0.01 * * *$ & \\
\hline \multirow[t]{2}{*}{ Type of additional examination } & $-0.87(-1.19,-0.55)^{* * *}$ & $-0.18(-0.45,0.10)$ & 0.12 \\
\hline & SD: $2.40 * * *$ & SD: $0.50 * * *$ & \\
\hline \multirow[t]{2}{*}{ Chance of additional examination (per \%) } & $-0.05(-0.06,-0.04)^{* * *}$ & $-0.03(-0.05,-0.01)^{* * *}$ & 0.12 \\
\hline & SD: $0.08 * * *$ & SD: $0.05^{* * *}$ & \\
\hline Log likelihood & -1824.52 & -335.42 & \\
\hline Pseudo R-squared & 0.30 & 0.16 & \\
\hline Number of observations & 3795 & 540 & \\
\hline Number of individuals & 248 & 36 & \\
\hline
\end{tabular}

$* * *, * *, *$ Significance at $1 \%, 5 \%, 10 \%$ level; SD: standard deviation; $+p$ Value was estimated in a joint model with interaction terms. 


\begin{tabular}{|c|c|c|c|c|c|c|c|c|}
\hline \multirow[t]{3}{*}{ Attribute } & \multicolumn{4}{|c|}{ Routine EEG Registration } & \multicolumn{4}{|c|}{ Routine MEG Registration } \\
\hline & \multirow{2}{*}{$\begin{array}{l}\text { Presumed level } \\
\text { for EEG }\end{array}$} & \multicolumn{3}{|c|}{ (Part-worth) Utilities } & \multirow{2}{*}{$\begin{array}{l}\text { Presumed } \\
\text { level for MEG }\end{array}$} & \multicolumn{3}{|c|}{ (Part-worth) Utilities } \\
\hline & & $\begin{array}{l}\text { Total } \\
(\mathrm{N}=289) \\
\end{array}$ & $\begin{array}{l}\text { Males } \\
(\mathrm{N}=130)\end{array}$ & $\begin{array}{l}\text { Females } \\
(\mathrm{N}=159)\end{array}$ & & $\begin{array}{l}\text { Total } \\
(\mathrm{N}=289)\end{array}$ & $\begin{array}{l}\text { Males } \\
(\mathrm{N}=130)\end{array}$ & $\begin{array}{l}\text { Females } \\
(\mathrm{N}=159)\end{array}$ \\
\hline $\begin{array}{l}\text { Way of measuring } \\
\text { brain activity }\end{array}$ & Electro cap & -0.017 & -0.16 & 0.05 & Helmet & 0.22 & -0.36 & -0.09 \\
\hline Duration & $70 \min$ & -1.26 & -0.7 & -1.4 & $60 \mathrm{~min}$ & -1.08 & -0.6 & -1.2 \\
\hline $\begin{array}{l}\text { Freedom of } \\
\text { movement }\end{array}$ & $\begin{array}{l}\text { Minor } \\
\text { movements } \\
\text { possible }\end{array}$ & 0 & 0 & 0 & $\begin{array}{l}\text { No } \\
\text { movements } \\
\text { possible }\end{array}$ & -1.84 & -1.36 & -2.14 \\
\hline Travel time * & 60 minutes & -0.90 & -1.2 & -1.2 & $60 \min$ & -0.9 & -1.2 & -1.2 \\
\hline $\begin{array}{l}\text { Type of additional } \\
\text { examination * }\end{array}$ & $\begin{array}{l}\text { EEG after sleep } \\
\text { deprivation }\end{array}$ & -0.94 & -0.64 & -1.12 & $\begin{array}{l}\text { EEG after } \\
\text { sleep } \\
\text { deprivation }\end{array}$ & -0.94 & -0.64 & -1.12 \\
\hline $\begin{array}{l}\text { Chance of } \\
\text { additional } \\
\text { examination }\end{array}$ & $\begin{array}{l}50 \text { out of } 100 \\
\text { patients }\end{array}$ & -2.85 & -2.50 & -2.00 & $\begin{array}{l}20 \text { out of } 100 \\
\text { patients }\end{array}$ & -1.14 & -1.00 & -0.80 \\
\hline $\begin{array}{l}\text { Overall utility } \\
\text { score }\end{array}$ & & -5.97 & -5.20 & -5.67 & & -6.12 & -5.16 & -6.55 \\
\hline
\end{tabular}

Figure 3.2 Example of (hypothesized) comparison of overall utility s cores between routine EEG and MEG *Level is constant for both recording methods

\section{Discussion}

To the best of our knowledge, this is the first study to investigate patients' preferences for EEG techniques in the primary diagnosis of epilepsy. This study was designed to identify to what extent important attributes of diagnostic procedures in epilepsy affected preferences for a procedure and to determine the relative importance of these attributes.

The overall fit of the model, as assessed with McFadden's pseudo $R^{2}$, was 0.28 and, when excluding respondents who failed the test-retest exercise, it increased to 0.30 . Both represent a decent model fit for a dis discrete choice model, as a pseudo $R^{2}$ of 0.30 represents $a R^{2}$ of approximately 0.6 for the equivalent $R^{2}$ of a linear regression model. ${ }^{52}$ Our results indicate that respondents would prefer a test with a short duration, a short travel time, in which minor movements are possible, the electric activity of the brain should be recorded by either a sensor net or an electro cap, a low chance of additional examination and, when additional examinations are necessary, it should preferably be 24-h EEG.

When looking at the relative importance weights, two attributes stand out: "chance of additional examinations" and "freedom of movement". The attribute "chance of additional examination" was expected to have a large influence as it relates to the sensitivity of a recording, which is often shown to be of great impact in preference studies. $^{29,54,55}$ Regarding "freedom of movement", we anticipated the fact that 
patients would prefer recordings in which minor movements are possible. However, beforehand, it was not entirely evident to assume that it would be the most influential factor in determining patients' preferences. Given these importance weights, one could argue that, albeit the presumed higher sensitivity and shorter duration of MEG, it is not straightforward to assume that, in general, patients would prefer MEG over routine EEG as shown in Figure 3.2. The comparison between both types of additional examinations, EEGsd and 24-h EEG, is more clear-cut. For additional diagnostics, this study suggests that patients have a strong preference for 24-h EEG over EEGsd, which could be explained by the fact that sleep deprivation may induce seizures and, therefore, patients might try to avoid it. In current practice, 24-h EEG is often used in patients with mental illness or children. However, this study suggests that 24-h EEG could be a more patient-friendly alternative for other patients as well. All parameters contained substantial heterogeneity, which indicates differences in the relative importance of the attributes between respondents. A part of the heterogeneity could be explained by the interaction between males and females regarding the attribute "freedom of movement" and by the interaction regarding the level "electro cap" between respondents who successfully completed the test-retest exercise and those who failed the test-retest exercise. These interactions highlight the fact that preferences could be substantially different between subgroups and even between individuals within a subgroup. For example, differences between males and females regarding "freedom of movement" could have an impact on their preferred diagnostic modality. Other factors explaining the heterogeneity in preferences could not be identified from the available data.

This study has several limitations. First, as Kempenhaeghe and UMCU are centers noted for expertise in the field of epilepsy, often the most complex or severe patients are being referred to these institutes. This is also supported by the fact that a relatively large percentage of the respondents (56.7\%) had experience with 24-h EEG, which is regularly only used in a specific subset of the patients. Therefore, selection of the sample could potentially be a problem, and our findings may only be limited and generalizable to the overall epilepsy care within The Netherlands.

Second, we encountered a response rate of $30.1 \%$ which is moderately low. Response rates to postal questionnaires are approximately $62 \%{ }^{56}$; however, regarding DCEs, large variations are reported ranging from $10 \%$ to $98 \% .{ }^{57,58}$ Some respondents reported difficulties when filling in the questionnaire (i.e. cognitive burden or too much attributes to take into account) which could be an explanation for the low response rate. The DCE method can be cognitively demanding which could lead to biased results by reducing respondents' ability to make a well-considered choice between different scenarios, especially because epilepsy is often associated with cognitive impairments. ${ }^{41,59}$ However, stratified analyses between respondents who perceived the task as relatively difficult and respondents who perceived the task as relatively easy did not show any significant differences. Furthermore, no differences were found between patients with different educational levels. Lastly, in our sample, a 
reasonable number of respondents (21.8\%) reported experience with MEG recording, which is relatively high as MEG is currently only used as presurgical evaluation instrument or in scientific research. In addition, a reasonable amount of respondents (24.2\%) reported to have not been diagnosed with routine EEG. Hence, given that in 2012, according to the Dutch Taskforce for Epilepsy Surgery, approximately 180 patients were referred for presurgical evaluation and the fact that a routine EEG is recommended as a first step of the diagnostic process in all European guidelines, ${ }^{11-13}$ recall bias is likely to be present within our sample. However, we have to rely on data as reported by respondents.

In conclusion, our study suggests that the way brain activity is measured, duration of the recording, freedom of movement, travel time, type of additional examination, and chance of additional examination are all important attributes in determining patients' preference for epilepsy diagnostics. The attributes with the largest impact on patients' preferences are freedom of movement and type of additional examination. Translated to clinical practice, this means that MEG is not necessarily more patient-friendly than a routine EEG, although an external validation study, comparing revealed preferences in patients experiencing both routine EEG and MEG, would be required to confirm this finding. Furthermore, regarding additional diagnostics, it can be concluded that patients have a strong preference for 24-h EEG over EEGsd, which could help physicians in medical decision-making. Additionally, bearing the substantial heterogeneity within the parameters in mind, it is important to take individual preferences into account in medical decision-making to improve epilepsy diagnostics. We believe that this study can be a valuable contribution to patient centered care within epilepsy diagnostics. Understanding and incorporating patients' preferences in the diagnostic trajectory of epilepsy (i.e. preferred type of additional examination) could improve the lengthy and burdensome process which is often associated with the diagnosis of epilepsy. ${ }^{10}$ In addition, incorporating patients' preferences into medical decision-making has been shown to significantly impact their willingness to use health care services. ${ }^{28-30}$

Recent studies, however, have revealed a disparity between patients' preferences and those of physicians. ${ }^{60,61}$ For most conditions, physicians underestimated the impact of intervention characteristics on patients' decision-making. ${ }^{61}$ Moreover, although it has been shown that a majority of physicians identified patient preferences as the most important general ethical guideline for surrogate decision-making, their medical decision-making relied on a variety of factors including prognosis, costs, patients' pain and suffering, and what was best for the patient overall (which closely resembles their preferences). ${ }^{62}$

In the Netherlands, the unit price of a MEG recording is about 200 Euros, whereas the unit price of a 24-h EEG or an EEGsd recording is about 160 Euros (price index year 2011). ${ }^{63}$ While the MEG recording is more expensive at first sight, the costeffectiveness (i.e., value for money) of this modality in epilepsy diagnostics will highly depend on the quantity and quality of the data delivered by the MEG recording 
compared to the data of a $24 \mathrm{~h}$ EEG and EEGsd recording. Although Colon et al. ${ }^{22}$ examined the additional value of MEG compared to EEGsd, future research should try to identify the informational value of the various diagnostic tests in relation to their financial implications as no studies have yet been performed on routine EEG, EEGsd, 24-h EEG, and MEG in a head-to-head comparison. Furthermore, future research should try to identify sources of heterogeneity between patients with epilepsy and the importance of incorporating these individual preferences in medical decisionmaking. 


\section{References}

1. Jacoby A, Baker GA. Quality-of-life trajectories in epilepsy: a review of the literature. Epilepsy Behav 2008;12:557-71.

2. Taylor RS, Sander JW, Taylor RJ, Baker GA. Predictors of health-related quality of life and costs in adults with epilepsy: a systematic review. Epilepsia 2011;52:2168-80.

3. Kotsopoulos IA, Evers SM, Ament AJ, de Krom MC. Estimating the costs of epilepsy: an international comparison of epilepsy cost studies. Epilepsia 2001;42:634-40.

4. Kotsopoulos IA, Evers SM, Ament AJ, Kessels FG, de Krom MC, Twellaar M, Metsemakers JF, Knottnerus AJ. The costs of epilepsy in three different populations of patients with epilepsy. Epilepsy Res 2003;54:131-40.

5. Ngugi AK, Kariuki SM, Bottomley C, Kleinschmidt I, Sander JW, Newton CR. Incidence of epilepsy: a systematic review and meta-analysis. Neurology 2011;77:1005-12.

6. Ngugi AK, Bottomley C, Kleinschmidt I, Sander JW, Newton CR. Estimation of the burden of active and lifetime epilepsy: a meta-analytic approach. Epilepsia 2010;51:883-90.

7. Neligan A, Hauser WA, Sander JW. The epidemiology of the epilepsies. In: Vinken PJ, Bruyn GW, editors. Handbook of Clinical Neurology,.2012;107:113-33.

8. Smith D, Defalla BA, Chadwick DW. The misdiagnosis of epilepsy and the management of refractory epilepsy in a specialist clinic. QJM 1999;92:15-23.

9. Chadwick D, Smith D. The misdiagnosis of epilepsy. BMJ 2002;324:495-6.

10. Benbadis S. The differential diagnosis of epilepsy: a critical review. Epilepsy Behav 2009;15:15-21.

11. Evidence NIfC. NICE guidelines: the diagnosis and management of the epilepsies in adults and children in primary and secondary care In: NICE, editor. ; 2012 (London).

12. Network SIG. Diagnosis and management of epilepsy in adults. Edinburgh: SIGN; 2003.

13. Epilepsie NvvnNLt. Epilepsie; Richtlijnen voor diagnostiek en behandeling. Werkgroep Richtlijnen Epilepsie; 2006.

14. Noachtar S, Remi J. The role of EEG in epilepsy: a critical review. Epilepsy Behav 2009;15:22-33.

15. Flink R, Pedersen B, Guekht AB, Malmgren K, Michelucci R, Neville B, Pinto F, Stephani U, Ozkara C; Commission of European Affairs of the International League Against Epilepsy: Subcommission on European Guidelines. Guidelines for the use of EEG methodology in the diagnosis of epilepsy. International League Against Epilepsy: commission report. Commission on European Affairs: Subcommission on European Guidelines. Acta Neurol Scand 2002;106: 1-7.

16. Rajna P, Veres J. Correlations between night sleep duration and seizure frequency in temporal lobe epilepsy. Epilepsia 1993;34:574-9.

17. Scalise A, Desiato MT, Gigli GL, Romigi A, Tombini M, Marciani MG, Izzi F, Placidi F. Increasing cortical excitability: a possible explanation for the proconvulsant role of sleep deprivation. Sleep 2006;29:1595-8.

18. Matos G, Andersen ML, do Valle AC, Tufik S. The relationship between sleep and epilepsy: evidence from clinical trials and animal models. J Neurol Sci 2010;295:1-7.

19. Frucht MM, Quigg M, Schwaner C, Fountain NB. Distribution of seizure precipitants among epilepsy syndromes. Epilepsia 2000;41:1534-9.

20. Malow BA. Sleep deprivation and epilepsy. Epilepsy currents, 4. American Epilepsy Society; 2004 193-5.

21. Nakken KO, Refsland G, Lillestolen KM, Solaas MH. Seizure-precipitating factors in epilepsy-what do patients report? Tidsskr Nor Laegeforen 2005;125:2172-4.

22. Colon AJ, Ossenblok P, Nieuwenhuis L, Stam KJ, Boon P. Use of routine MEG in the primary diagnostic process of epilepsy. J Clin Neurophysiol 2009;26:326-32.

23. Heers M, Rampp S, Kaltenhäuser M, Pauli E, Rauch C, Dölken MT, Stefan H. Detection of epileptic spikes by magnetoencephalography and electroencephalography after sleep deprivation. Seizure 2010;19:397-403.

24. Ossenblok P, de Munck JC, Colon A, Drolsbach W, Boon P. Magnetoencephalography is more successful for screening and localizing frontal lobe epilepsy than electroencephalography. Epilepsia 2007;48:2139-49.

25. Bridges JF, Jones C. Patient-based health technology assessment: a vision of the future. Int J Technol Assess Health Care 2007;23:30-5. 
26. Facey K, Boivin A, Gracia J, Hansen HP, Lo Scalzo A, Mossman J, Single A. Patients' perspectives in health technology assessment: a route to robust evidence and fair deliberation. Int J Technol Assess Health Care 2010;26:334-40.

27. Ryan M. Discrete choice experiments in health care. BMJ 2004;328:360-1.

28. Dominitz JA, Provenzale D. Patient preferences and quality of life associated with colorectal cancer screening. Am J Gastroenterol 1997;92:2171-8.

29. Marshall DA, Johnson FR, Kulin NA, Ozdemir S, Walsh JM, Marshall JK, Van Bebber S, Phillips KA. How do physician assessments of patient preferences for colorectal cancer screening tests differ from actual preferences? A comparison in Canada and the United States using a stated-choice survey. Health Econ 2009;18:1420-39.

30. Woolf SH. The best screening test for colorectal cancer-a personal choice. N Engl J Med 2000;343: 1641-3.

31. Lancsar E, Louviere J. Conducting discrete choice experiments to inform healthcare decision making: a user's guide. Pharmacoeconomics 2008;26:661-77.

32. Ryan M, Gerard K. Using discrete choice experiments to value health care programmes: current practice and future research reflections. Appl Health Econ Health Policy 2003;2:55-64.

33. Essers BA, Dirksen CD, Prins MH, Neumann HA. Assessing the public's preference for surgical treatment of primary basal cell carcinoma: a discrete-choice experiment in the south of the Netherlands. Dermatol Surg 2010;36:1950-5.

34. Hol L, de Bekker-Grob EW, van Dam L, Donkers B, Kuipers EJ, Habbema JD, Steyerberg EW, van Leerdam ME, Essink-Bot ML. Preferences for colorectal cancer screening strategies: a discrete choice experiment. Br J Cancer 2010;102:972-80.

35. Paczkowski MM, Kruk ME, Tessema F, Tegegn A, Galea S. Depressive symptoms and posttraumatic stress disorder as determinants of preference weights for attributes of obstetric care among Ethiopian women. PLoS One 2012;7:e46788.

36. Regier DA, Diorio C, Ethier MC, Alli A, Alexander S, Boydell KM, Gassas A, Taylor J, Kellow C, Mills D, Sung L. Discrete choice experiment to evaluate factors that influence preferences for antibiotic prophylaxis in pediatric oncology. PLoS One 2012;7:e47470.

37. Manjunath R, Yang J-C, Ettinger AB. Patients' preferences for treatment outcomes of add-on antiepileptic drugs: a conjoint analysis. Epilepsy Behav 2012;24:474-9.

38. de Bekker-Grob EW, Hol L, Donkers B, van Dam L, Habbema JD, van Leerdam ME, Kuipers EJ, EssinkBot ML, Steyerberg EW. Labeled versus unlabeled discrete choice experiments in health economics: an application to colorectal cancer screening. Value Health 2010;13:315-23.

39. Ryan M, Farrar S. Using conjoint analysis to elicit preferences for health care. BMJ 2000;320:1530-3.

40. Louviere JJ, Hensher DA, Swait JD. Stated choice methods: analysis and applications. Cambridge University Press; 2000.

41. Bryan S, Dolan P. Discrete choice experiments in health economics. For better or for worse? Eur J Health Econ 2004;5:199-202.

42. Electrical geodesics I. HydroCel geodesic sensor nets. http://www.egi.com/research- divisionresearch-products/sensor-nets.

43. Binnie CD, Stefan H. Modern electroencephalography: its role in epilepsy management. Clin Neurophysiol 1999;110:1671-97.

44. Walter WG, Hodge R, Hutchinson J. Discussion on recent advances in the EEG diagnosis of epilepsy. Proc R Soc Med 1951;44:315.

45. Reed Johnson F, Lancsar E, Marshall D, Kilambi V, Mühlbacher A, Regier DA, Bresnahan BW, Kanninen $B$, Bridges JF. Constructing experimental designs for discrete-choice experiments: report of the ISPOR Conjoint Analysis Experimental Design Good Research Practices Task Force. Value Health 2013;16: 3-13.

46. Hiligsmann M, Dellaert BG, Dirksen CD, van der Weijden T, S. G., Reginster J, et al. Patients' preferences for osteoporosis drug treatment: a discrete choice experiment. Manuscript presented at the 5e LolaHESG Conference, 23-24 May 2013, Landgoed Vennendal (the Netherlands); 2013.

47. Kessels R, Jones B, Goos P, Vandebroek M. The usefulness of Bayesian optimal designs for discrete choice experiments. Appl Stoch Model Bus Ind 2011;27:173-88. 
48. Alayli-Goebbels AF, Dellaert BG, Knox SA, Ament AJ, Lakerveld J, Bot SD, Nijpels G, Severens JL. Consumer preferences for health and nonhealth outcomes of health promotion: results from a discrete choice experiment. Value Health 2013;16:114-23.

49. Ryan M, Skatun D. Modelling non-demanders in choice experiments. Health Econ 2004;13:397-402.

50. Bech M, Kjaer T, Lauridsen J. Does the number of choice sets matter? Results from a web survey applying a discrete choice experiment. Health Econ 2011;20:273-86.

51. Bridges JF, Hauber AB, Marshall D, Lloyd A, Prosser LA, Regier DA, Johnson FR, Mauskopf J. Conjoint analysis applications in health-a checklist: a report of the ISPOR Good Research Practices for Conjoint Analysis Task Force. Value Health 2011;14:403-13.

52. Hensher DA, Rose JM, Greene WH. Applied choice analysis: a primer. Cambridge University Press; 2005.

53. Malhotra NK, Birks DF. Marketing research: an applied approach. Pearson Education Limited; 2007.

54. Marshall DA, Johnson FR, Phillips KA, Marshall JK, Thabane L, Kulin NA. Measuring patient preferences for colorectal cancer screening using a choice-format survey. Value Health 2007;10:415-30.

55. Nayaradou M, Berchi C, Dejardin O, Launoy G. Eliciting population preferences for mass colorectal cancer screening organization. Med Decis Making 2010;30:224-33.

56. Asch DA, Jedrziewski MK, Christakis NA. Response rates to mail surveys published in medical journals. J Clin Epidemiol 1997;50:1129-36.

57. van Helvoort-Postulart D, van der Weijden T, Dellaert BG, de Kok M, von Meyenfeldt MF, Dirksen CD. Investigating the complementary value of discrete choice experiments for the evaluation of barriers and facilitators in implementation research: a questionnaire survey. Implement Sci 2009;4:10.

58. Ryan M. Using conjoint analysis to take account of patient preferences and go beyond health outcomes: an application to in vitro fertilisation. Soc Sci Med 1999;48:535-46.

59. Helmstaedter C, Kurthen M, Lux S, Reuber M, Elger CE. Chronic epilepsy and cognition: a longitudinal study in temporal lobe epilepsy. Ann Neurol 2003;54:425-32.

60. Montgomery A, Fahey T. How do patients' treatment preferences compare with those of clinicians? Qual Health Care 2001;10:i39-43.

61. Mühlbacher AC, Juhnke C. Patient preferences versus physicians' judgement: does it make a difference in healthcare decision making? Appl Health Econ Health Policy 2013:1-18.

62. Torke AM, Moloney R, Siegler M, Abalos A, Alexander GC. Physicians' views on the importance of patient preferences in surrogate decision-making. J Am Geriatr Soc 2010;58:533-8.

63. The Dutch Healthcare Authority. DBC zorgproducten tariefapplicatie. http://dbc- zorgproductentarieven.nza.nl/nzaZpTarief/Welkom.aspx; 2013. 


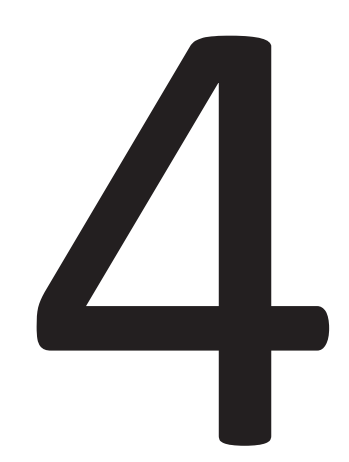

Side-effects of antiepileptic drugs:

The economic burden

RJA de Kinderen, SMAA Evers, R Rinkens, D Postulart, CI Vader, HJM Majoie, AP Aldenkamp

Seizure 2014;23:184-190 


\section{Abstract}

\section{Purpose}

Antiepileptic drugs are potentially an effective treatment for patients with epilepsy. However, side-effects are common. The negative consequences of side-effects can lead to treatment ranging from minor care to very expensive hospitalization. This cost analysis has been conducted to provide insight into the costs of side-effects due to antiepileptic drugs in the Netherlands from a societal perspective.

\section{Methods}

Health care, patient and family and other use of resources for five different categories of sideeffects were measured by means of a questionnaire.

\section{Results}

Based on data from 203 chronic epilepsy patients, the overall societal costs of common sideeffects in 2012 are estimated to be $€ 20,751(\mathrm{Cl}: 15,049-27,196)$ per patient per year in the base case analysis. These societal costs exist of: mean health care costs $(€ 4,475)$, mean patient and family costs (i.e. informal care) $(€ 10,472)$ and mean other costs (i.e. productivity losses) $(€ 5,747)$. Examining the different categories of side-effects separately, ranging from the most to the least expensive category, the cost estimates were as follows: other side-effects $(€ 13,228)$, behavioral side-effects $(€ 9,689)$, general health side-effects $(€ 7,454)$, cognitive side-effects $(€ 7,285)$ and cosmetic side-effects $(€ 2,845)$ per patient per year. Subgroup analyses showed significant differences in costs between patients using monotherapy and those using polytherapy when looking at cognitive and cosmetic side-effects.

\section{Conclusion}

These estimates should be considered in the overall assessment of the economic impact of a pharmacotherapy. 


\section{Introduction}

Antiepileptic drugs (AEDs) are potentially an effective treatment for patients with epilepsy. Treatment failure and poor adherence are, however, very common in patients experiencing side-effects due to AEDs. In approximately $25 \%$ of the patients, side-effects lead to treatment discontinuation ${ }^{1-3}$ and have a substantial negative impact on the quality of life of patients with epilepsy. ${ }^{3,4}$ Furthermore, the negative consequences of side-effects can have a significant impact on the lives of relatives and friends of the patient, but also on society as a whole.

Side-effects of AEDs that commonly occur are memory problems, fatigue, tremors, gastrointestinal complaints, osteoporosis, depression, drowsiness, dizziness, weight change, nausea etc. ${ }^{5}$ Common side-effects due to AEDs will lead to medical interventions ranging from minor care to very expensive specialist care and hospital admissions. In addition to these health care costs, patient and family costs (i.e. informal care) and costs in other sectors (e.g. loss of employment) can be substantial.

Numerous studies have calculated the economic burden of epilepsy in many different countries. ${ }^{6-12}$ Only one study assessed the direct costs of severe idiosyncratic reactions due to antiepileptic drugs of hospitalized patients from an institutional perspective. ${ }^{13}$ None of these studies focused on the economic burden of the commonly occurring side-effects due to AEDs in their analyses. In order to accurately reflect the total societal economic burden of epilepsy, costs related to side-effects should be included in the analysis.

The overall objective of this study is, therefore, to estimate the annual health care costs, the patient and family costs and costs in other sectors of commonly occurring side-effects due to AEDs in the Netherlands.

\section{Methods}

All patients with epilepsy using antiepileptic drugs, who visited the tertiary epilepsy center Kempenhaeghe, Heeze, the Netherlands, between September 2011 and November 2011, received a patient information letter by mail including an invitation to complete a questionnaire $(\mathrm{N}=1386)$. The letter contained information about the content of the questionnaire and the purpose of the study. Furthermore, the letter stated that participation was completely voluntarily and that participant's data would be anonymously analyzed and reported. Participants were invited to complete the questionnaire only when they had experienced side-effects during the previous 12 months. For young children and patients with severe mental retardation, proxy measures were taken. The questionnaire could either be completed digitally via the internet or on paper. All participants (patients, parents or caregivers) gave their informed consent. 


\section{Questionnaire}

The SIDe effects of AED treatment (SIDAED) ${ }^{14}$ was used as the basis for the questionnaire. The original 10 side-effect categories of the SIDAED were compressed into four categories, in order to focus on the most common side-effects and to condense the questionnaire. The categories used in this study were: cognition (e.g. memory problems, more slow thinking, feeling drowsy or sleepy etc.), cosmetic (e.g. weight problems, skin rash, surplus saliva etc.), behavioral (e.g. depressed, irritated, pressurized or excitable etc.) and general health (i.e. general CNS, vision, headache, gastrointestinal, sexuality/menses complaints). A fifth category was added ('other' complaints) to allow patients to report side-effects that they could not classify within one of the aforementioned categories.

The questionnaire starts with some basic demographic questions (age, sex, education, employment, and AED usage). Then the first subdivision of the questionnaire starts with the question 'Have you experienced any cognitive side-effects, such as slow reaction or memory and concentration problems, during the last 12 months?' If not, the questions about cognitive side-effects can be skipped and the patient can go on to the subdivision of the questionnaire about cosmetic-related side-effects. Then the patients will answer the question whether or not they have encountered cosmetic side-effects, such as skin rash, hair loss or weight gain, during the last 12 months, etc. If a patient has experienced cognitive side-effects, he or she is asked to describe their complaints and to fill out all the questions about resource use belonging to this specific category. All categories of the questionnaire are dealt with in this way. The questions about resource use are exactly the same for all five categories. As the cost analysis is performed from a societal perspective, this implies that the measurement of resources has to be broad, i.e. all costs related to use of resources have to be included, irrespective of who pays. As a result, resource use in health care, patients and family and other sectors due to side-effects are measured. Health care usage includes visits to the general practitioner, specialists, psychologists, alternative health care, paramedics (i.e. dietician, speech therapist or physiotherapist), admission to a general, academic or psychiatric hospital or to an epilepsy center, care received, including day care, occupational care, social services, home care, prescribed and overthe-counter (OTC) medication for side-effects. Patient and family resource use includes informal care and out of pocket expenses. The sector 'other resource use' includes loss of productivity and absenteeism from activities of daily life.

\section{Analyzing costs}

The total costs were estimated using a bottom-up approach, where information on each element of service used was multiplied by an appropriate standardized unit cost and summed to provide an overall total cost. ${ }^{15}$ The index year for the study was 2012 (consumer price index (inflation) number: 111.39$)^{16}$ and standard cost prices were derived from the Dutch Manual for Costing ${ }^{17}$ or (if not available) calculated mean cost 
prices according to providers were used. In accordance with these guidelines, medication costs were calculated based on daily defined dosage taken from the Dutch pharmaceutical therapeutic compass combined with the Dutch consumer reimbursement price of medication. ${ }^{18}$ When data on medication was diverse, lowest cost prices for the specific medication were used.

Costs of informal care and absenteeism from daily activity were calculated using standardized cost prices based on shadow prices. 'Shadow pricing' is a method used to impute values on cost items for which no market prices are available. In this case, the minimum wage rate of the Netherlands was used to estimate the cost of informal care provided by relatives or friends of the patient and losses of daily activity. For out of pocket payments, costs declared by the patient were used.

Productivity losses from paid work were quantified in terms of net cumulative number of days of sick leave over a period of 12 months. In the case of partial sick leave, we assumed that subjects were $100 \%$ productive during the hours of partial work resumption. Productivity losses were calculated based on the Human Capital Approach (HCA). The cumulative number of calendar days of sick leave was converted into work-hour equivalents based on the mean number of work-hours per week registered by the patients. The costs of production losses were calculated by multiplying the number of sick leave hours by the estimated reference cost of production loss for an employee per hour of sick leave. ${ }^{17}$

Despite the usual skewness in the distribution of costs, arithmetic means are generally considered to be the most appropriate measures for describing cost data. ${ }^{19,20}$ Therefore, arithmetic means are presented. However, to check for sample uncertainty, non-parametric bootstrapping was used. This method is based on random sampling, with replacement based on the participants' individual data. ${ }^{21}$ Nonparametric bootstrapping avoids the need to make assumptions about the shape of the distribution, such as normality, and instead uses the observed distributions of the cost data in the study being analyzed. The non-parametric bootstrap resample method was applied with 1000 replications in this study. The bootstrap replications were used to calculate $95 \%$ confidence intervals around the costs, based on the 2.5 th and 97.5 th percentiles. The data on costs were analyzed using the statistical package SPSS 20.0 (SPSS, Chicago, IL, USA) and MS-Excel 2010.

\section{Subgroup and sensitivity analyses}

Subgroup analyses were performed to check for differences between groups. Firstly, a combination of AEDs can produce negative interaction effects which can lead to sideeffects. A separate subgroup analysis was, therefore, performed to check for differences between patients on monotherapy and patients on polytherapy. Secondly, as there might be differences between AED and side-effects over time, another subgroup analysis was performed to check for differences between children and adults. 
Additionally, a sensitivity analysis was performed to check for uncertainty. As different methods can be used to put a value on productivity losses, we corrected for the methodological uncertainty by calculating productivity losses on the basis of the friction cost method (FCM) instead of the HCA. The FCM is based on the assumption that an organization needs a certain time span to replace the absent worker by another worker. The definitive number of days absent from work is limited to the duration of the friction period, determined in the Netherlands to be 23 weeks. ${ }^{17}$

\section{Results}

In total, 210 patients from the tertiary epilepsy center completed the questionnaire. Although we asked to fill out the questionnaire only when patients had experienced any side effects during the last 12 months, in seven, neither side-effects nor costs were described and so they were excluded from the analysis. The characteristics of all included participants are shown in Table 4.1.

Table 4.1 Demographic characteristics ( $N=203)$.

\begin{tabular}{lll}
\hline & $N$ & $\%$ \\
\hline Age mean (range) & $38(2-81)$ & 50.2 \\
Female & 102 & 9.9 \\
Education & & 6.4 \\
No education & 20 & 0.5 \\
Too young for school & 13 & 8.4 \\
At primary school & 1 & 8.9 \\
Special education & 17 & 15.3 \\
Primary education & 18 & 5.4 \\
Lower level secondary education & 31 & 25.6 \\
Higher level secondary education & 11 & 19.7 \\
Secondary vocational education & 52 & 27.6 \\
Higher education & 40 & 30 \\
Paid work & 56 & 33 \\
Number of different AED per patient & & 23.6 \\
One & 61 & 10.8 \\
Two & 67 & 3.4 \\
Three & & 1.5 \\
Four & & 0 \\
Five & 48 & 0.5 \\
Six & 22 & 7 \\
Seven & 3 & 0 \\
\hline
\end{tabular}

Most of the participants (85\%) had experienced some kind of general health sideeffect due to AEDs during the last 12 months. Cognitive side-effects are the second most commonly reported problems among participants (77\%). Behavioral side-effects 
appear in $68 \%$ of the participants. Cosmetic side-effects occur in $42 \%$ of the participants and $7 \%$ report other side-effects.

Table 4.2 lists the reference prices per unit and the arithmetic mean total costs per category. Table 4.3 shows the bootstrapped mean total costs of all categories and their 95\% confidence intervals. The arithmetic mean costs are comparable to the bootstrapped mean costs. On the whole, most costs are generated in the patient and family sector compared to costs in health care and other sectors. Overall, informal care is the main cost driver accounting for $51 \%$ of the total annual costs per patient. Production losses account for $43 \%$ of the other sector costs, but only for $12 \%$ of the overall total costs. However, only $28 \%$ of the patients had a paid job and were, therefore, responsible for the costs of production losses. Patients without a paid job generated the costs of daily routine losses.

Table 4.3 shows the differences in costs between the side-effect categories. As can be seen, admissions to a general or academic hospital often occur in the general health side-effects category and behavioral problems lead to numerous admissions to the epilepsy center. Resource use related to daycare is mostly present in the general health category; homecare is an expensive factor in the cognitive, behavioral and general health side-effects category. Prescribed medication only leads to high costs in the category 'other'. This is in particular due to one person who underwent treatment with Teriparatide injections for osteoporosis during the last 12 months (total costs > 5000 Euros). ${ }^{18}$

Overall, total costs per patient in the category 'other side-effects' are the highest. These are based on a small group $(n=15)$ of which a few patients experienced side effects due to AEDs which specifically led to high costs of informal care and many losses in daily routine.

\section{Subgroup and sensitivity analyses}

Table 4.3 shows the bootstrapped means of the subgroup analyses performed. Firstly, mean costs are calculated separately for patients on monotherapy and those on polytherapy. Of the 203 participants, 61 were on monotherapy. Overall, mean costs due to side-effects per patient are considerably higher in the polytherapy group. For the categories cognitive and cosmetic side-effects, the mean costs for a polytherapy patient is three times more than for a patient on monotherapy (€2,679 vs. $€ 9,012$ resp. $€ 1,002$ vs. $€ 3,389$ ). The difference between mean costs for monotherapy and polytherapy in the categories behavioral side-effects and general health side-effects are less prominent and statistically not significant ( $€ 7,154$ vs. $€ 10,498$ and $€ 6,135$ vs. $€ 8,029)$. As there are only two patients on monotherapy which have experienced any other side-effects, bootstrapping is impossible and presenting the arithmetic mean would be incorrect. 


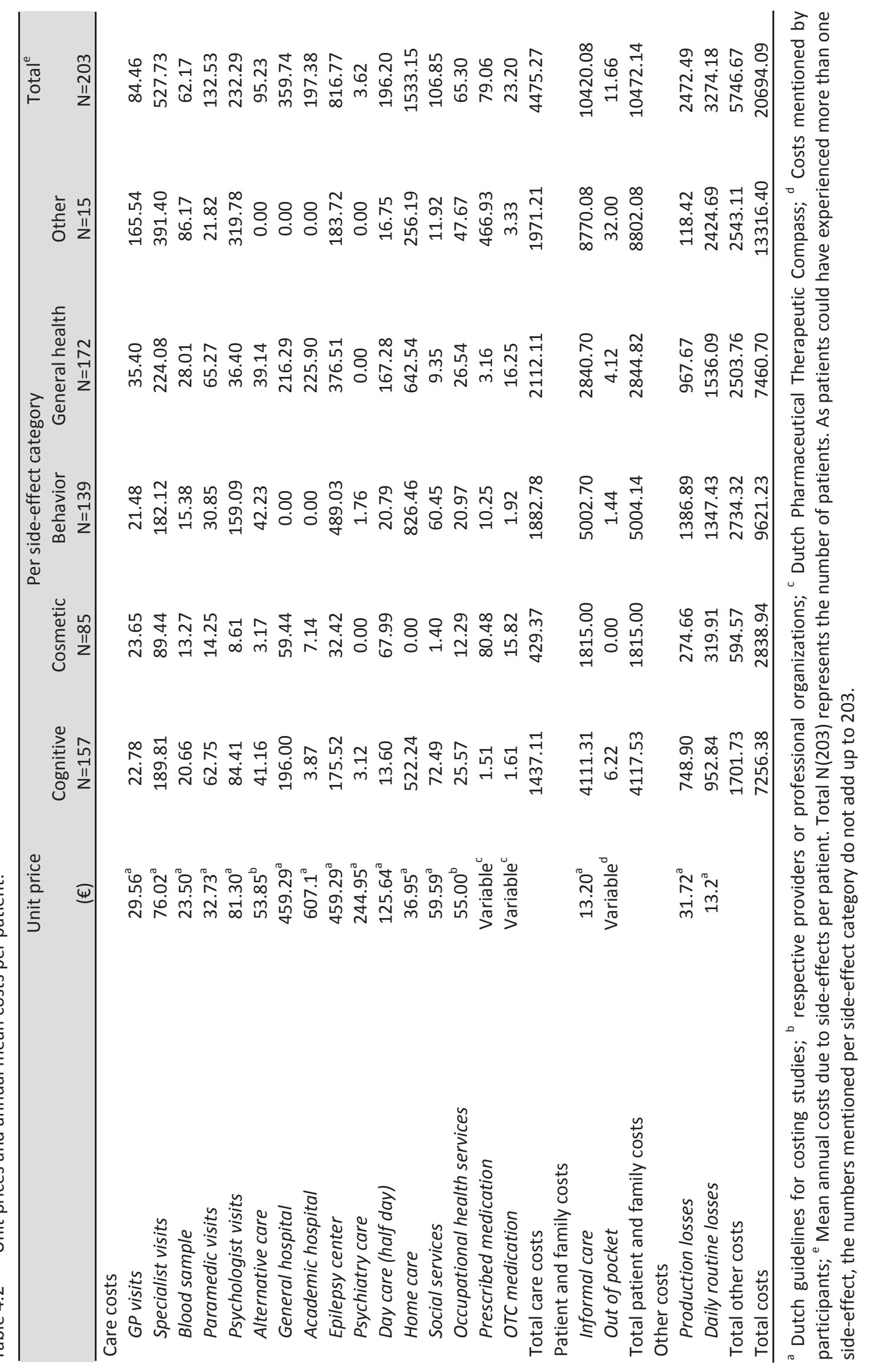




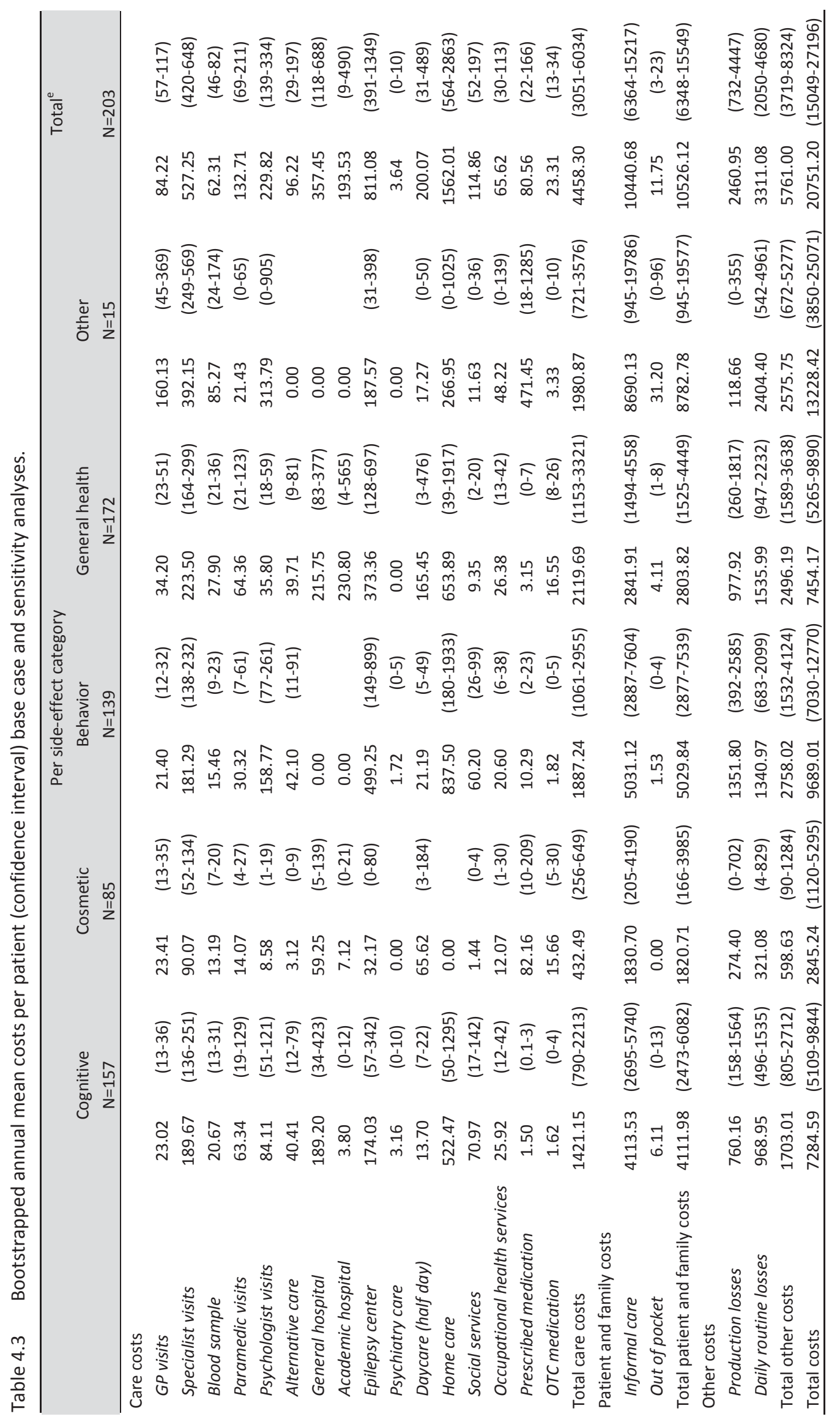




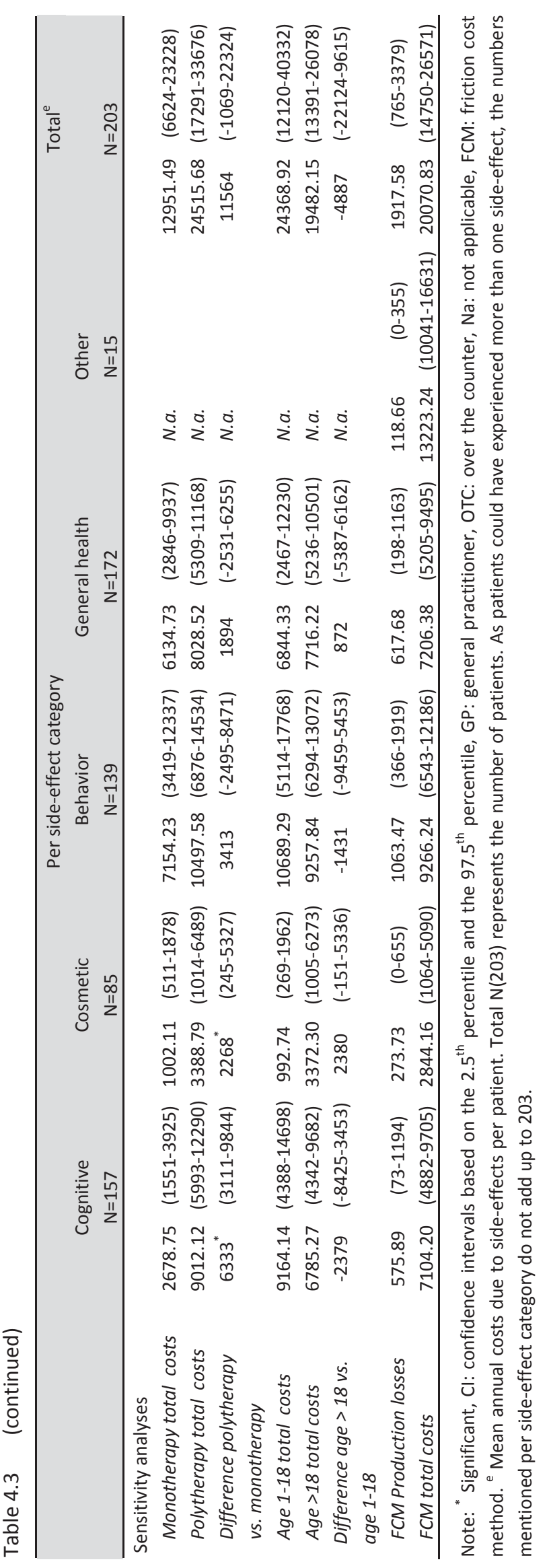


Secondly, total mean costs were calculated separately for children and adolescent patients (aged 1-18) and for adult patients older than 18 years. Of all 203 participants, 50 patients were between 1-18 years of age. Overall, total mean costs per patient due to side-effects seem to be higher in children and adolescents. However, in the categories cosmetic and general health, side-effect costs were higher in the adult group. No statistically significant differences in costs were found in the different sideeffect categories. Again, showing the bootstrapped or the arithmetic mean of this subgroup analysis in the other side-effects category would be incorrect as this group is small (4 kids vs. 11 adults).

Furthermore, Table 4.3 shows the result of the bootstrapped means of the sensitivity analysis. When using the FCM instead of the HCA to calculate productivity losses in a sensitivity analysis, total costs of all side-effects decreased to $€ 20,071$. The FCM was applied in four patients who exceeded the maximum length of the friction period (23 weeks). More specifically, costs of cognitive side-effects decreased from $€ 7,285$ to $€ 7,104$, of behavioral side-effects from $€ 9,689$ to $€ 9,266$ and of general side-effects from $€ 7,454$ to $€ 7,206$. As the patients who exceeded the maximum length of the friction period did not report any cosmetic or other side-effects, these costs remained the same.

\section{Discussion}

Based on the health care, patient and family, and other resource use reported by 203 epilepsy patients with side effects, the general societal costs of common side-effects in 2012 due to antiepileptic drugs is estimated to be $€ 20,751$ per patient. Patient and family costs were on the whole higher than the cost of health care or the costs in the other sectors. Examining the different categories of common side-effects separately, other side-effects generated the most $(€ 13,228)$ and cosmetic side-effects the least costs $(€ 2,845)$ per patient per year. Behavioral effects generate the second highest costs per patient per year $(€ 9,689)$ closely followed by costs of general health sideeffects $(€ 7,454)$ and cognitive side-effects $(€ 7,285)$. Furthermore, several subgroup analyses showed only significant differences in costs between patients on monotherapy and patients on polytherapy within the cognitive and the cosmetic sideeffects category.

Although we did not find any studies focusing on the cost of common side-effects due to AEDs, it can be stated that our results are in line with other cost of illness literature. For example, Pugliatti et al. ${ }^{22}$ estimated the cost of epilepsy in Europe based on a review with economic modeling and also concluded that costs outside formal health care expenditure were the single most costly resource item for epilepsy in Europe, productivity losses being the most dominant cost category. Strzelczyk et al. ${ }^{23}$ reviewed twelve studies on indirect costs and these ranged between $12 \%$ and $85 \%$ of the total costs. These results are highly depending on the way of measuring this cost category. 
Although in our study most costs were again generated outside the health care sector, the most dominant category was informal care. The euro value of the burden associated with informal care may have been even higher than estimated, as we assumed a minimum wage for all informal care providers.

It can be stated that the economic burden of common side-effects is considerable compared with the costs of the disease itself. For example, estimates show total costs of epilepsy per patient per year of only $€ 4,292$ in the Netherlands ${ }^{24}, € 8,275$ in Sweden $^{25}, € 13,412$ in Denmark ${ }^{26}, € 7,738$ in Germany. ${ }^{27}$

Our results confirm that from an economic perspective, it is very important that the treatment of epilepsy patients is primarily concerned with balancing seizure control and adverse effects. Side-effects exert a high burden, not only for society, but also for the individual patient. One study showed that patients are willing to pay €879 (£709) ( $€ 1=€ 1.24,2012$ exchange rate) per month to achieve $100 \%$ seizure reduction and no adverse effects. The same study showed that patients are willing to pay only €216 (f174) per month for a drug that provided seizure freedom but also caused hair loss. ${ }^{28}$ We have to consider certain limitations of our study. Firstly, only subjective complaints and related health care resource use reported by patients were used. It has been proven that the frequency at which side-effects of antiepileptic drugs are reported in a given population is dependent on the method of assessment. Reliance on unstructured interviews or spontaneous reporting underestimates the burden of toxic effects of antiepileptic drugs, whereas use of screening measures, such as questionnaires or checklists, can result in overestimation. ${ }^{29}$ Furthermore, caution should be exercised when relying on patient-recorded cost data ${ }^{30}$, recall bias is to be expected especially with a long time horizon (12 months). However, using a cost questionnaire enabled us to obtain actual data on patient and family costs, including informal care, which cannot be captured through other sources such as hospital databases and patient records.

Secondly, since we did not include a control group in our analyses, we were not able to compare health care costs in people with epilepsy with the health care costs of those without epilepsy. It has, however, already been proven that patients suffering from epilepsy are associated with higher expenditure, both because of being more likely to have expenses and because the average expense is higher. ${ }^{26,31}$ Yoon et al. (2009) calculated the health care expenditure of people with epilepsy and compared it to that of non-epileptics. They found that the average excessive direct health care expenditure due to epilepsy was USD $\$ 4,523$. Furthermore, they found that adults with epilepsy received significantly more informal care than people without epilepsy. Jennum et al. (2011) showed that the direct net annual health care and indirect costs were $€ 14,575$ for patients and $€ 1,163$ for controls, i.e. a consequent excess cost of $€ 13,412$.

Thirdly, our study has a potential bias towards severely affected patients at an epilepsy center as only $30 \%$ of the patients were on monotherapy. 
In conclusion, this study, despite its limitations, demonstrates the economic burden induced by patients with antiepileptic side-effects from a societal perspective in the Netherlands. Assuming that more than 80,000 people have epilepsy in the Netherlands $s^{32}$ and that the demonstrated costs can be as high as $€ 20,751$ per patient per year, side-effects due to antiepileptic drugs will have a major impact on health care costs in the Netherlands. These figures should be considered along with the costs associated with drug acquisition, delivery, and treatment of all clinical successes and failures, in the overall assessment of the economic impact of pharmacotherapy 


\section{References}

1. Kwan P, Brodie MJ. Early identification of refractory epilepsy. N Engl J Med. 2000 Feb 3;342(5):314-9.

2. Perucca P, Carter J, Vahle V, Gilliam FG. Adverse antiepileptic drug effects: toward a clinically and neurobiologically relevant taxonomy. Neurology. 2009;72:1223-9.

3. Uijl SG, Uiterwaal CS, Aldenkamp AP, Carpay JA, Doelman JC, Keizer K, et al. Adjustment of treatment increases quality of life in patients with epilepsy: a randomized controlled pragmatic trial. Eur J Neurol. 2009;16:1173-7.

4. Luoni C, Bisulli F, Canevini MP, De Sarro G, Fattore C, Galimberti CA, et al. Determinants of healthrelated quality of life in pharmacoresistant epilepsy: results from a large multicenter study of consecutively enrolled patients using validated quantitative assessments. Epilepsia. 2011;52:2181-91.

5. Carpay JA, Aldenkamp AP, van Donselaar CA. Complaints associated with the use of antiepileptic drugs: results from a community-based study. Seizure. 2005;14:198-206.

6. Begley CE, Annegers JF, Lairson DR, Reynolds TF, Hauser WA. Cost of epilepsy in the United States: a model based on incidence and prognosis. Epilepsia. 1994;35:1230-43.

7. Beran RG. The burden of epilepsy for the patient: the intangible costs. Epilepsia. 1999;40 Suppl 8: 40-3.

8. Griffiths RI, Schrammel PN, Morris GL, Wills SH, Labiner DM, Strauss MJ. Payer costs of patients diagnosed with epilepsy. Epilepsia. 1999;40:351-8.

9. Heaney DC, Sander JW, Shorvon SD. Comparing the cost of epilepsy across eight European countries. Epilepsy Res. 2001;43:89-95.

10. Kotsopoulos IA, Evers SM, Ament AJ, de Krom MC. Estimating the costs of epilepsy: an international comparison of epilepsy cost studies. Epilepsia. 2001;42:634-40.

11. Nsengiyumva G, Druet-Cabanac M, Nzisabira L, Preux PM, Vergnenegre A. Economic evaluation of epilepsy in Kiremba (Burundi): a case-control study. Epilepsia. 2004;45:673-7.

12. Thomas SV, Sarma PS, Alexander M, Pandit L, Shekhar L, Trivedi C, et al. Economic burden of epilepsy in India. Epilepsia. 2001;42:1052-60.

13. Schlienger RG, Oh PI, Knowles SR, Shear NH. Quantifying the costs of serious adverse drug reactions to antiepileptic drugs. Epilepsia. 1998;39 Suppl 7:S27-32.

14. Uij SG, Uiterwaal CS, Aldenkamp AP, Carpay JA, Doelman JC, Keizer K, et al. A cross-sectional study of subjective complaints in patients with epilepsy who seem to be well-controlled with anti-epileptic drugs. Seizure. 2006;15:242-8.

15. Drummond MF, Sculpher MJ, Torrance GW, O'Brien BJ, Stoddart GL. Methods for the economic evaluation of health care programs. Oxford: Oxford University Press; 2005.

16. CBS. STATLINE derived at September $2012 \mathrm{http}: / /$ statline.cbs.nl/StatWeb/publication/?VW=T\&DM= SLNL\&PA=71311NED\&D1=0,2,4,6\&D2=0-1,61,70,87,108,137,145,172,176,221-222,230,255, I\&D3=(I34) - \&HD=081020-1310\&HDR=T\&STB=G1,G2: Centraal Bureau voor de Statistiek; 2012 [cited September, 2012].

17. Tan SS, Bouwmans CAM, Rutten FFH, Hakkaart-van Roijen L. Update of the Dutch Manual for Costing in Economic Evaluations. Int J Technol Assess. 2012;28:152-8.

18. College van Zorgverzekeringen. Farmacotherapeutisch Kompas: Medisch farmaceutische voorlichting Amstelveen: CVZ; 2012.

19. Ramsey S, Willke R, Briggs A, Brown R, Buxton M, Chawla A, et al. Good research practices for costeffectiveness analysis alongside clinical trials: the ISPOR RCT-CEA Task Force report. Value in health : the journal of the International Society for Pharmacoeconomics and Outcomes Research. 2005;8: 521-33.

20. Thompson SG, Barber JA. How should cost data in pragmatic randomised trials be analysed? Bmj. 2000;320:1197-200.

21. Briggs AH, Wonderling DE, Mooney CZ. Pulling cost-effectiveness analysis up by its bootstraps: a nonparametric approach to confidence interval estimation. Health Econ. 1997;6:327-40.

22. Pugliatti M, Beghi E, Forsgren L, Ekman M, Sobocki P. Estimating the cost of epilepsy in Europe: a review with economic modeling. Epilepsia. 2007;48:2224-33.

23. Strzelczyk A, Reese JP, Dodel R, Hamer HM. Cost of epilepsy: a systematic review. Pharmacoeconomics. 2008;26:463-76. 
24. Kotsopoulos IA, Evers SM, Ament AJ, Kessels FG, de Krom MC, Twellaar M, et al. The costs of epilepsy in three different populations of patients with epilepsy. Epilepsy Res. 2003;54:131-40.

25. Bolin K, Lundgren A, Berggren F, Kallen K. Epilepsy in Sweden: health care costs and loss of productivity-a register-based approach. The European journal of health economics : HEPAC : health economics in prevention and care. 2012;13:819-26.

26. Jennum P, Gyllenborg J, Kjellberg J. The social and economic consequences of epilepsy: a controlled national study. Epilepsia. 2011;52:949-56.

27. Strzelczyk A, Nickolay T, Bauer S, Haag A, Knake S, Oertel WH, et al. Evaluation of health-care utilization among adult patients with epilepsy in Germany. Epilepsy Behav. 2012;23:451-7.

28. Lloyd A, Mclntosh E, Price M. The importance of drug adverse effects compared with seizure control for people with epilepsy: a discrete choice experiment. Pharmacoeconomics. 2005;23:1167-81.

29. Baker GA, Camfield C, Camfield P, Cramer JA, Elger CE, Johnson AL, et al. Commission on Outcome Measurement in Epilepsy, 1994-1997: final report. Epilepsia. 1998;39:213-31.

30. Goossens ME, Rutten-van Molken MP, Vlaeyen JW, van der Linden SM. The cost diary: a method to measure direct and indirect costs in cost-effectiveness research. J Clin Epidemiol. 2000;53:688-95.

31. Yoon D, Frick KD, Carr DA, Austin JK. Economic impact of epilepsy in the United States. Epilepsia. 2009;50:2186-91.

32. Wallace H, Shorvon S, Tallis R. Age-specific incidence and prevalence rates of treated epilepsy in an unselected population of 2,052,922 and age-specific fertility rates of women with epilepsy. Lancet. 1998;352:1970-3. 
Part

Economic evaluations 


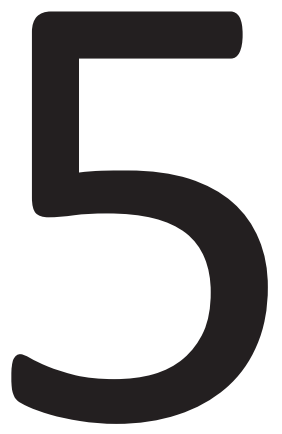

\section{Methods in Economic Evaluations of Treatments for Intractable Epilepsy: A Systematic Review}

RJA de Kinderen, D Postulart, SMAA Evers, DAJE Lambrechts, AP Aldenkamp, HJM Majoie 


\section{Abstract}

\section{Background}

Intractable epilepsy is associated with significant morbidity, impaired quality of life, mortality and substantial costs. The increasing number of treatment options and the inherent high costs have fostered the development of economic evaluations in the area of refractory epilepsy. As economic evaluations are used as a basis for decision-making by governments and other influential bodies, it is important that the quality is beyond question.

\section{Objective}

This study aimed to provide an overview and a quality check of published full economic evaluations of interventions for patients with intractable epilepsy in order to inform those seeking to assess the economic impact of epilepsy treatment.

\section{Data sources}

A systematic review of English articles using PubMed and the Centre for Reviews and Dissemination Database (2000-2011). Search terms included cost-effectiveness, cost-utility, cost-benefit, therapy resistant epilepsy, drug resistant epilepsy, intractable epilepsy, and refractory epilepsy.

\section{Study selection}

Only full economic evaluations of treatments in refractory epilepsy were included. Independent selection of studies was performed by three authors.

\section{Data extraction methods}

Independent extraction of articles by four authors using predefined epidemiological and health economic data fields, including general quality indicators.

\section{Results}

Eventually, 12 articles were selected for inclusion. The methodological quality of these articles was assessed on five issues: (1) the perspective from which the analysis was performed, (2) the type of economic appraisal and the study design, (3) the outcome measures of costs and consequences and the associated time horizon in which these outcomes can be expected, (4) the discounting of costs and outcomes, and (5) the performance of comprehensive sensitivity analyses.

\section{Conclusions}

We systematically checked and discussed the included articles with regard to these five issues and found that certain items were often poorly described, overlooked, or not taken into account at all. We agree with previous reviews about the difficulty of comparing results of economic evaluations in epilepsy due to heterogeneity in methods and concepts used. 


\section{Background}

Although in the majority of cases epilepsy is treatable with anti-epileptic drugs (AEDs), about $30 \%$ of patients suffer from intractable epilepsy. ${ }^{1}$ These patients depend heavily on informal care (family and friends) and on health care professionals (neurologists, social workers, psychologists etc.). Complications due to intractable epilepsy result in frequent hospitalizations and many of these patients will eventually live in an institution. When looking at different groups of epilepsy patients, intractable epilepsy is associated with the second highest costs per patient per year, after surgical candidates. $^{2-3}$ It has been estimated that countries are spending up to $1 \%$ of their total national health care budget on epilepsy ${ }^{4}$ and that the total costs of epilepsy in Europe were $€ 15.5$ billion in $2004 .^{5}$ Besides the economic burden, epilepsy is also associated with a reduced Quality Of Life (QOL). ${ }^{6}$

The increasing number of treatment options and the high costs associated with intractable epilepsy fostered the development of economic evaluations in this field. Economic evaluations compare costs and consequences of health care interventions. For patients with refractory epilepsy, add-on AEDs or non-pharmacological treatments are available (e.g. Ketogenic Diet, Vagus Nerve Stimulator). The goal of good health policy making is to achieve health benefits and therefore to 'produce' health. In order to make good choices in health care, policymakers try to find a balance between costs and effects of interventions. Economic evaluations provide a solution for this assessment, as they provide information about the relative efficiency of choosing between alternative interventions. In a "full" economic evaluation, the additional benefits of a health care program are weighed against the additional costs compared to a reference program. For example, an economic evaluation will reveal whether a new AED is 'good value for money' compared to existing alternatives.

Economic evaluations are important at different levels of decision-making. The Ministry of Health can use economic evaluations, for example, to determine which interventions are eligible for insurance packages. In some countries, economic assessments are now of such high importance that a drug will not be refunded if no economic evaluation is conducted. But also at the level of national healthcare services or at the level of the individual patient, economic evaluations can help in decisionmaking. The principle of economic evaluation is simple: determine the cost/benefit ratio and select the intervention for which this ratio is the lowest. Although the principle seems simple, performing a good economic evaluation study is no sinecure. As influential decisions depend on the results of economic evaluations, the methodological quality of the assessment should be high. Unfortunately this is not always the case. A decade ago, a review was published on the methodological quality of economic evaluations of anticonvulsants. ${ }^{7}$ Due to the large heterogeneity of methodologies, the results of different economic evaluations were not comparable, making an assessment of costs and effects between interventions impossible. This review has been conducted in order to check whether the quality of economic 
evaluations in the field of epilepsy has improved and to inform those seeking to assess the economic impact of epilepsy treatment. The aim of this review will be to present an overview and a methodological quality check of published full economic evaluations of all healthcare interventions for patients with intractable epilepsy.

\section{Essentials of economic evaluations}

Simply measuring the costs of an intervention will not tell us whether this intervention is cost-effective. A cheap intervention may represent poor value for money if it has little effect on outcome. The general approach of an economic evaluation is to compare the consequences of health care programmes with their costs. A costeffectiveness ratio (ICER) is then calculated for each treatment being assessed, where the denominator reflects the incremental gain in effects, benefits or utilities, and the numerator reflects the additional cost of achieving that health gain.

\section{Types of economic evaluation}

There are three types of full economic evaluation, namely: Cost-Benefit Analysis (CBA), Cost-Effectiveness Analysis (CEA) and a Cost-Utility Analysis (CUA). In a CBA, attempts are made to value the consequences of programmes in monetary terms, so as to make them commensurate with the costs. Potentially, this is the broadest programme for justifying the costs. However, expressing benefits in monetary terms is often hampered by measurement problems. In a CEA, the consequences of programmes are measured in the most appropriate natural effects of physical units, such as 'seizure-free days' or 'seizure-free patients'. The main argument for these clinical measures is the relative ease of measurement and interpretation. One of the major limitations of the CEA and its disease-specific outcome is that it does not permit comparisons with interventions evaluated in other studies. In a CUA, the consequences of programmes are adjusted by health state preference scores or utility weights; that is, states of health associated with the outcomes are valued relative to one another. The most common measure of consequences in a CUA is the qualityadjusted life-year (QALY). ${ }^{8}$ A QALY incorporates effects in terms of both quality of life (utilities) and survival (life-years gained). In contrast to the outcome measure of the CEA, it is a generic outcome measure. To calculate QALYs, it is necessary to represent health on a scale where death and full health are assigned values of 0 and 1 , respectively. As there are many ways of obtaining utilities to calculate QALYs, it is important to look at the methods used or the questionnaire chosen. A disease-specific quality of life measure, for example, is specific to an individual disease or condition and includes dimensions relevant to that disease (e.g. epilepsy-specific QOL measure: QOLIE-89). A generic quality of life measure is a comprehensive measure of health 
that has a broad range of components and is applicable across different patient groups (e.g. EuroQoL). ${ }^{9}$

\section{Study design}

There are two study designs to conduct an economic evaluation, namely trial-based or model-based design. In trial-based economic evaluations, data on resource use and effectiveness are collected simultaneously, for example, alongside a randomized trial. The benefits of this approach are that results reflect actual data from real patients, and the internal validity of the data could be assessed through controlled clinical conditions. The disadvantage of this approach is that it may not be possible to generalize the treatment pathways and patient samples to the larger population. To overcome this disadvantage, it is possible to model the costs and outcomes of different treatments. Model-based economic evaluations provide a means of bringing all clinical, costs and quality of life data together in one model by using techniques of epidemiology or decision analysis. Therefore, its results can be generalized in terms of the larger population. Lifetime costs and outcomes can then be estimated.

Decision-tree models present a sequence of decisions and chance events over time. Each chance event is assigned a probability. Alternative decision strategies are evaluated by calculating their average consequences. A limitation of decision-tree models is that the probability of each chance event is static. In chronic diseases, the probability of chance events changes with age, health status, and time. Therefore, state-transition models are often used; these allocate and reallocate subjects into previously defined health states. ${ }^{10}$ Transitions occur from one health state to another at defined time intervals according to the transition probabilities. Through simulation, the number of subjects in the population passing through each state at each point of time can be estimated, as well as the lifetime costs and effects.

\section{Perspective of analysis}

The perspective assumed in an economic analysis is important. The perspective indicates from which point of view an economic evaluation is being considered and determines which costs will be examined, and thus which conclusions can be drawn from any particular economic evaluation. A programme that looks unattractive from one viewpoint may look significantly better when other viewpoints are considered. Analytical viewpoints may include any or all of the following: the individual patient, the specific institution, the target group for specific services, the Ministry of Health budget, and the community or societal viewpoint. According to guidelines, an economic evaluation study has to be performed from the societal perspective. ${ }^{8}$ In this case, all costs including health care costs, costs of patient and family and costs in other sectors are to be measured, despite who benefits or pays for them. 


\section{Measurement and valuation of costs and consequences}

Health care costs of epilepsy are, for example, costs for AEDs, outpatient visits to physicians, pharmaceuticals, hospital admissions, laboratory investigations, etc. Costs of patient and family are include transportation expense to access care, cost for respite care, cost of safety equipment, special furniture, etc. Examples of costs in other sectors are costs of home help, costs of social worker visits, costs of productivity losses etc.

Once the important and relevant costs and consequences have been identified, they must be measured in appropriate physical and natural units. The sources and methods of valuation of costs, effects, benefits, and utilities should be clearly stated in an economic evaluation. All current and future programme costs are normally valued in constant currency of some base year, in order to remove the effects of inflation from the analysis. Additionally, as an individual will usually prefer benefits today to benefits tomorrow and costs tomorrow rather than costs today, time preference has to be built into estimates of both costs and effects to reflect their current values. This process of calculating the present value of future costs and effects, benefits and utilities is called discounting and is necessary when the time horizon is longer than 12 months. ${ }^{11}$

\section{Time horizon}

The choice of the time horizon is an important methodological consideration in economic evaluations. It should be long enough to capture the major health and economic consequences, both intended effects and unintended side-effects. Ideally it should have a lifetime horizon in order to capture the relevant outcomes in terms of the mortality and morbidity associated with epilepsy.

\section{Allowance for uncertainty}

Every economic evaluation will contain some degree of uncertainty, imprecision, or methodological controversy. Sensitivity analyses should, therefore, ideally be performed on both the effects and the costs in order to check the robustness of the results. Univariate sensitivity analysis involves sequentially testing the impact of changes in single variables on the study findings. Individual parameters are varied across a range that is either based on available evidence or according to a formally derived estimate. Multivariate sensitivity analysis involves testing more than one assumption at a time. Probability distributions for each variable to be tested may be used (i.e. probabilistic sensitivity analysis). Furthermore, non-parametric bootstrapping can be used to check sample uncertainty. Bootstrapping is a computerbased method for assigning measures of accuracy to sample estimates by constructing a number of random re-samples of the observed dataset. This provides an estimate of the shape of the distribution of those sample estimates. 


\section{Methods}

\section{Literature search}

A literature search was performed in PubMed and the Centre for Reviews and Dissemination databases (the Database of Abstracts of Review of Effects (DARE), the National Health Service Economic Evaluation Database (NHS EED) and the Health Technology Assessments (HTA) Database. A search in these databases is generally a reliable and valid strategy for finding economic evaluations. ${ }^{12-13}$ The search terms 'economic evaluation' or, 'cost-effectiveness' or, 'cost-utility' or, 'cost-benefit' and 'therapy resistant epilepsy' or, 'drug resistant epilepsy' or, 'intractable epilepsy' or, 'refractory epilepsy' were used in combination with each other. Citation tracking was conducted to complete the search.

\section{Eligibility criteria}

The review focused on full economic evaluations comparing different treatment options for patients with refractory epilepsy. We nonetheless excluded studies devoted to surgical treatment of epilepsy, for it is an option restricted to certain specific forms of epilepsy. In addition to the other reviews, only articles published in English during the period January 2000 - October 2011 were considered. Eligibility assessment was performed independently in a standardized manner by three reviewers. Disagreements between reviewers were resolved by consensus.

\section{Data collection process and quality check}

A 3-part checklist has been developed to enable a systematic quality check of the economic evaluation studies. This consists of a general part, an epidemiological part and an economic part. Furthermore, different checklists were used for model-based articles $^{14}$ and for trial-based articles ${ }^{15}$ as the two methods are not comparable in their methodology. The checklist was systematically used as a quality assessment of the included economic evaluations. Two neurologists ( $M M$ and $\mathrm{DL}$ ) scored the general part and the epidemiological part of the included articles and two Health Technology Assessment (HTA) experts (SE and DP) scored the general part and the economic part of the included articles. The first author (RK) scored the general, epidemiological and economic part of the included articles. As a consequence, all the articles were doublescored both on the epidemiological as well as on the economical part. Possible differences in scoring were discussed until consensus was reached.

This qualitative appraisal was conducted by considering the perspective, type of economic evaluation, study design, time horizon, credibility of study (sponsorship), measurement and valuation of costs and consequences, allowing for uncertainty and incremental analysis of costs and consequences. 


\section{Results}

\section{Search results}

A total of 130 publications were identified: 95 with PubMed, 35 with the Centre for Reviews and Dissemination databases (five with DARE, 20 with the NHS EED and 10 within the HTA Database). The flow chart of the literature search is presented in Figure 5.1.

After screening the titles and abstracts on inclusion criteria, 113 articles were excluded; 31 because no treatment was given, 16 were non-English articles, 15 were duplicates, ten were reviews, two were animal studies, 37 lacked an economic evaluation, and two articles because the form of epilepsy under research was not refractory epilepsy. Another five articles were eliminated from the analysis after reading. Despite what was suggested in the title or abstract, in all five cases, the article did not refer to a full economic evaluation. A total of 12 publications were finally available for this review. ${ }^{16-27}$

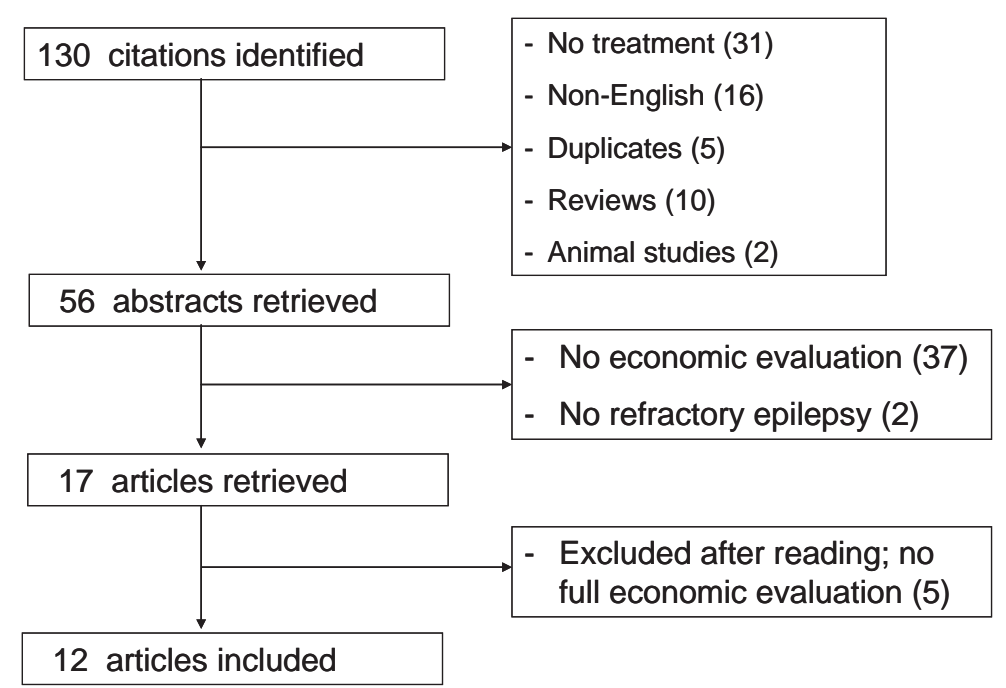

Figure 5.1 Flowchart of literature search.

\section{General characteristics}

The most recent study was from 2010, and the oldest study was published in 2003, using 2001 data. All economic evaluations were performed for five different countries. The countries of origin of the articles were: United Kingdom (eight articles), Canada (one article), the Netherlands (one article), Korea (one article) and United States of America (one article). 


\section{Perspective of Analysis}

Since the perspective of a study determines the categories of costs and consequences that will be examined and thus the conclusions drawn, economic analyses should explicitly mention the perspective of the study. Only eight of the studies in our sample did so (Table 5.1). It is, however, likely that the external source financing the study will give some indication of the perspective of the study. From the articles that did not mention the perspective of the study, most studies received funds from pharmaceutical companies. Overall, two studies ${ }^{20-21}$ were not funded by pharmaceutical companies at all. The authors of only one article claimed to have conducted their research based on a societal perspective, ${ }^{16}$ Their study results cannot, however, be used for resource allocation on the societal level as they have only included health care costs. Four articles mentioned that the analyses were performed from a United Kingdom National Healthcare Service (NHS) perspective ${ }^{17,20,25,27}$ and one from the Scottish NHS perspective. ${ }^{22}$ In one article it was mentioned that the analysis was based on the Canadian ministry of health perspective ${ }^{19}$ and in another that it was based on the third-party payer perspective. ${ }^{24}$

\section{Nature of economic appraisal}

This review includes four articles in which a CEA was performed, six articles in which a CUA was performed and another two articles that include both types of analyses (Table 5.1). The outcome measures used in the CEAs were number of seizure-free days, number of seizure-free patients, and a $50 \%$ or more seizure reduction. The outcome measures used in the CUA are QALYs gained.

\section{Study design}

In total, 10 articles used model-based economic evaluation to conduct their research (Table 5.1). Only two studies conducted a trial-based economic evaluation, of which one used a pre-experimental mirror-image design, the patients serving as their own controls, ${ }^{21}$ and the other one used a prospective observational design. ${ }^{23}$ Of the modelbased economic evaluations, five state-transition models, ${ }^{20,22,23,25,27}$ four decisiontrees, ${ }^{17,19,24,26}$ and one unspecified model were used. ${ }^{16}$

\section{Time horizon}

The time horizon of all included studies ranged from six months ${ }^{18}$ to lifetime ${ }^{16}$ (Table 5.1). Of the two trial-based articles, one had a time horizon of six months ${ }^{18}$ and the other 12 months. ${ }^{21}$. Among the model-based articles, four articles had a time horizon of only one year, ${ }^{17,19,23,24}$ another of two years ${ }^{26}$ and two articles had a time horizon of three years. ${ }^{25,27}$ Only two articles had a time horizon of 15 years ${ }^{20,22}$ and only one a lifetime time horizon. ${ }^{16}$ 


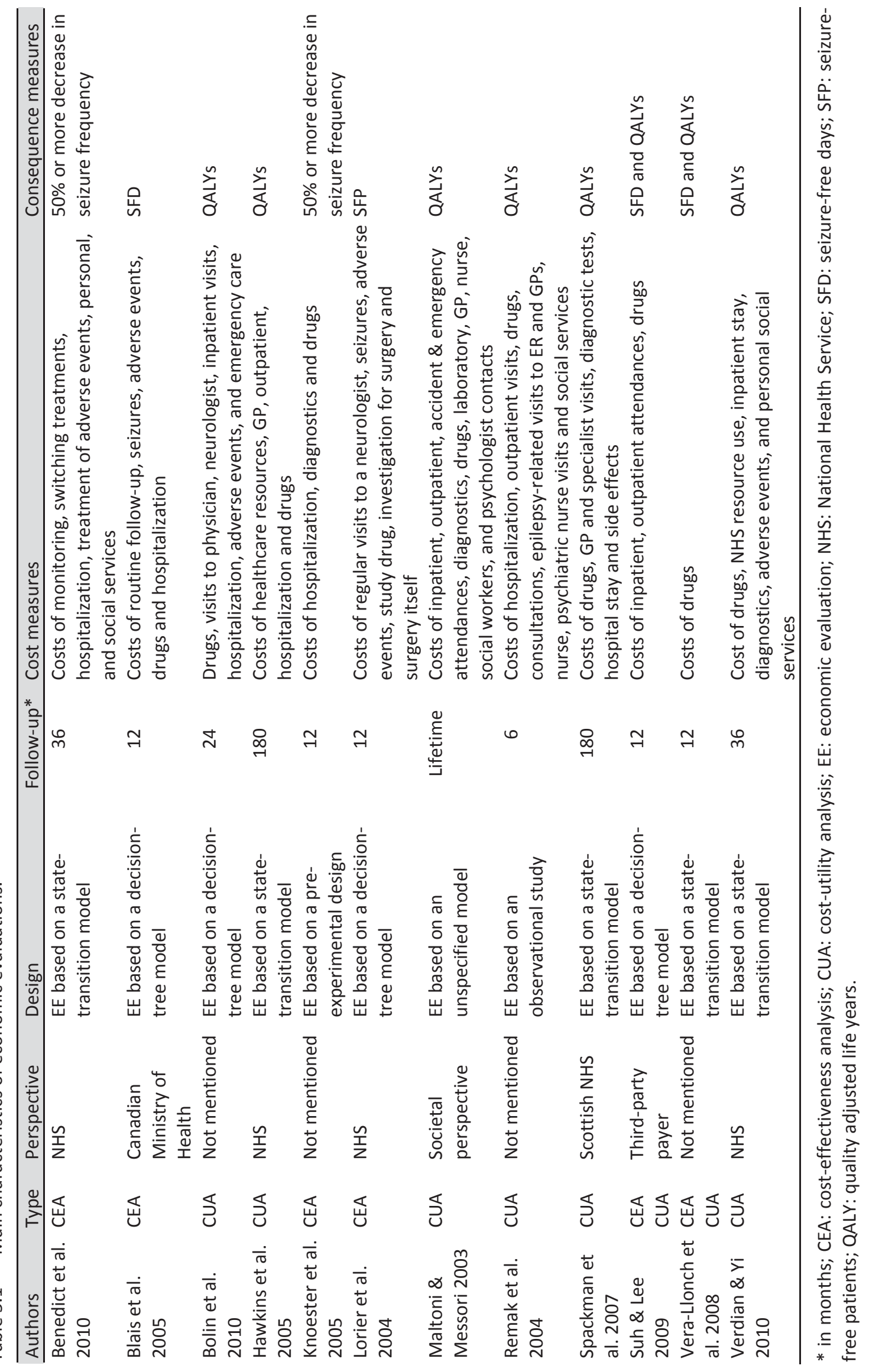




\section{Measurement and valuation of costs and consequences}

All studies included (some) healthcare costs. Neither patient and family costs nor costs in other sectors were included in articles. The range of costs included within the health care category differed widely between studies: from including only the cost of AEDs in the primary analysis ${ }^{23}$ to including costs of inpatient visits, outpatient visits, laboratory tests, diagnostics, GP visits, nurse visits, and psychologist contacts etc. ${ }^{16}$ (Table 5.1).

As mentioned above, different outcome measures were used in the articles. In two articles, consequences were expressed in $50 \%$ or more seizure reduction, ${ }^{21,25}$ in three articles 'seizure-free days' was the outcome measure ${ }^{19,23,24}$ and in one article the outcome measure was 'seizure-free patient'. ${ }^{17}$ Almost $70 \%$ of the articles (also) included QALYs as a measurement of consequences. ${ }^{16,18,20,22-24,26,27}$ In one article, ${ }^{24}$ the QOLIE-31 was used, which is an epilepsy-specific QOL measure; the results are, therefore, not comparable with the other included articles. Furthermore, it is important to look at whose preferences are taken into account when valuing health states, as patient and population values can differ significantly. ${ }^{9}$ Three studies ${ }^{18,20,22}$ used the utilities derived from the Euroqol-5D questionnaire (EQ-5D) which has been valued by the general population to calculate QALYs. Others ${ }^{16,26,27}$ used utilities from published literature which were obtained by the time trade off (TTO) and valued by patients. And in another article, ${ }^{23}$ utilities derived from a survey in which epilepsy patients value their own health on a scale from 0-100 were used.

\section{Allowance for uncertainty}

Discounting was not relevant for six studies included in the review, because the time horizon in each case was 12 months or less (Table 5.1). One study had a time horizon of 24 months and explicitly mentioned that no discounting was performed but gave no explanatory reason for this decision. ${ }^{26}$ Costs and benefits were discounted by four studies. ${ }^{16,20,22,27}$ Only costs were discounted in one article, ${ }^{25}$ as benefits in their study were measured at one moment in time. The discount rates for costs differed between $3 \%$ and $6 \%$ percent and for benefits ranged from $1.5 \%$ to $3.5 \%$.

All studies mentioned that a sensitivity analysis was performed to check the influence of the assumptions made and the robustness of the conclusions. Eight studies tested the differences in both costs and effects statistically. Of these articles, four used probabilistic sensitivity analysis, ${ }^{20,27}$ sometimes in combination with one-way sensitivity analysis. ${ }^{23,26}$ The other four articles used a one-way sensitivity analysis, ${ }^{19}$ sometimes in combination with a two-way analysis ${ }^{22}$ and at other times with a multiway analysis, ${ }^{16}$ or with the bootstrap method. ${ }^{25}$ One article did not mention the methods used for performing the sensitivity analysis, only that the analysis was 'extensive'. ${ }^{24}$ Some studies performed only a sensitivity analysis on the sampling variation by means of the bootstrap method. ${ }^{18,21}$ Finally, in one article, ${ }^{17}$ performing a 
sensitivity analysis was mentioned in the discussion, although the results of this analysis were not found by our team in tables or the written text.

\section{Main results of the studies}

The studies in this review evaluated a wide range of interventions (Table 5.2). One study compared three interventions, ${ }^{27}$ another four, ${ }^{25}$ another five ${ }^{18}$ and one study compared eight interventions. ${ }^{20}$ All calculated an ICER or an ICUR. In some studies, the ICER was expressed in costs per seizure-free day. ${ }^{19,23,24}$ Others expressed the ICER in costs per $50 \%$ or more seizure reduction ${ }^{21,25}$ or in cost per seizure-free patient. ${ }^{17}$ Almost $70 \%$ of the studies, however, expressed the ICUR in costs per QALY gained. ${ }^{16,18,20,22-24,26,27}$ Costs were expressed in US dollars, ${ }^{23,24}$ in Canadian dollars, ${ }^{19}$ in Euros, ${ }^{21,26}$ or in UK Pounds. ${ }^{16-18,20,22,25,27}$

The main findings of these studies are shown in Table 5.2. All included articles studied merely add-on AEDs compared to standard therapy, other add-on AEDs or placebo. Two articles compared rufinamide add-on with lamotrigine or with topiramate addon. ${ }^{25,27}$ Benedict calculated an ICER for rufinamide add-on of $£ 2,151$ per $1 \%$ increase in successfully treated patients with a seizure reduction of $50 \%$ or more, compared with lamotrigine add-on (topiramate was dominated). Verdian \& Yi calculated an ICUR for rufinamide add-on of $£ 20,538$ per QALY gained, compared with topiramate add-on (lamotrigine was dominated). ${ }^{27}$

Three articles compared all levetiracetam add-on with standard therapy. ${ }^{17,19,24}$ Blais et al. calculated an ICER for levetiracetam of $C \$ 80.70$ per seizure free day gained compared with standard therapy. ${ }^{19}$ Lorier et al. calculated an ICER for levetiracetam of $£ 5,301$ per seizure-free patient gained compared with standard therapy. ${ }^{17}$ Suh \& Lee calculated for levetiracetam an ICUR of $\$ 11,084$ per QALY gained compared with standard therapy. ${ }^{18}$

One article compared lacosamide add-on with standard therapy and calculated an ICUR of $€ 27,641$ per QALY gained. ${ }^{26}$ Another article compared Lamotrigine add-on with standard therapy and calculated an ICER of €954 per patient with $50 \%$ or more seizure reduction. ${ }^{21}$ There was one article comparing pregabalin add-on with standard therapy and calculated an ICUR of $\$ 52,893$ per QALY and an ICER of $\$ 28.45$ per seizure free day. ${ }^{23}$

Maltoni \& Messori ${ }^{16}$ compared topiramate add-on with standard therapy and Remak et al. ${ }^{18}$ compared topiramate add-on with clobazam, gabapentin, lamotrigine and with vigabatrin. Maltoni \& Messori calculated for topiramate an ICUR of $£ 21,353$ per QALY gained compared with standard therapy. ${ }^{16}$ Remak et al. calculated for topiramate an ICUR of $£ 7,869$ per QALY gained compared with vigabatrin (other AEDs were dominated). ${ }^{18}$ 


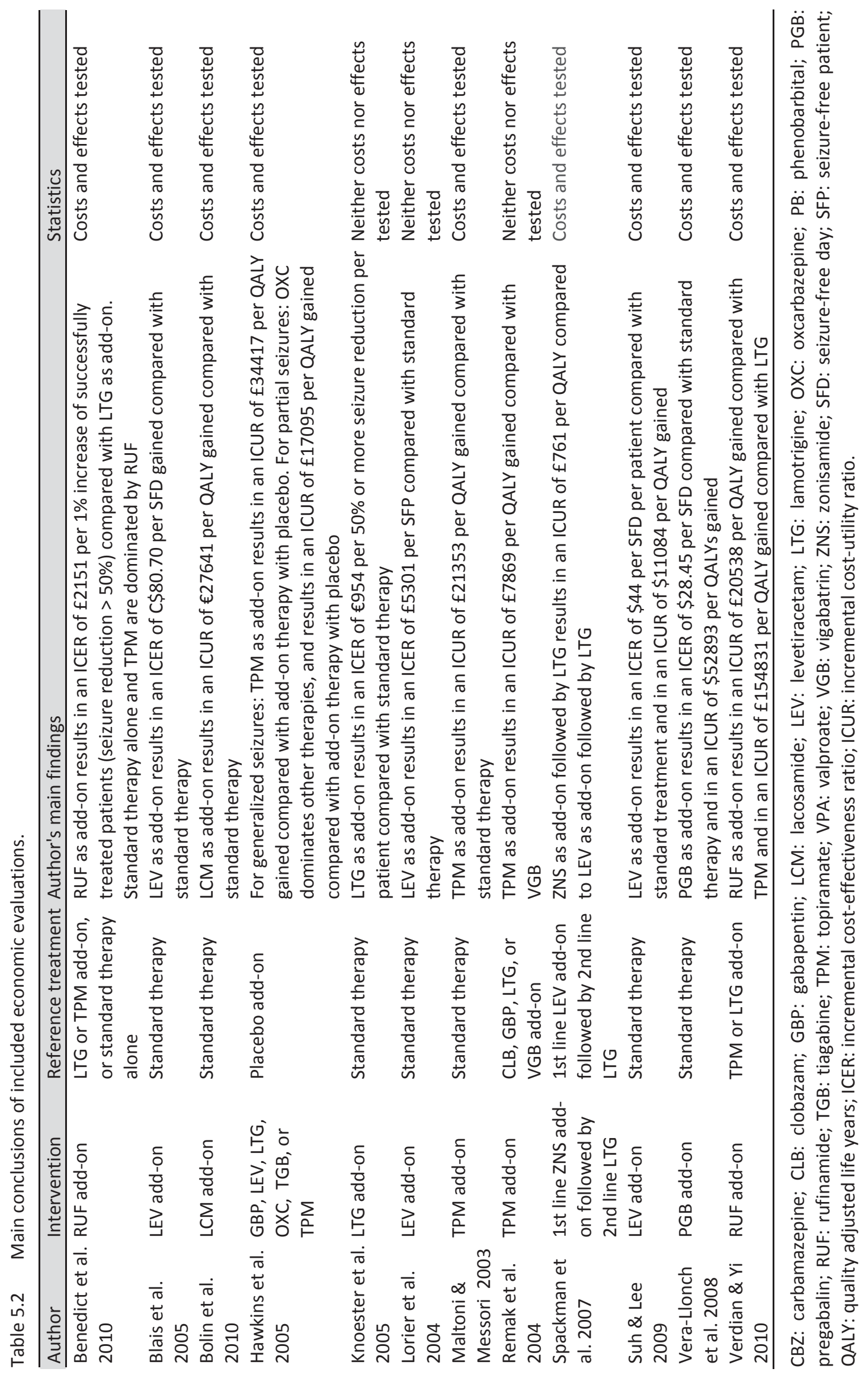


Hawkins et al. compared topiramate for generalized seizures with placebo add-on and calculated an ICUR of $£ 34,417$ per QALY gained. ${ }^{20}$ Furthermore, they compared different AEDs with placebo add-on for partial seizures and calculated an ICUR for oxcarbazepine of $£ 17,095$ per QALY gained (other AEDs were dominated).

Spackman et al. include switching among alternative AEDs and calculated an ICUR of f761 per QALY when first line add-on therapy with zonisamide is followed by second line lamotrigine compared with first line add-on therapy with levetiracetam is followed by second line lamotrigine. ${ }^{22}$ Therefore, the mean cost per QALY is a weighted average of different treatments.

\section{Discussion}

The increasing number of treatment options and the associated high costs have fostered the development of economic evaluations in the area of intractable epilepsy. As influential decisions depend on these evaluations, it is of utmost importance that the evaluations are of high quality. The aim of this review was to look at full economic evaluations of intractable epilepsy in order to check the quality and to inform those dealing with epilepsy treatment decision-making at the clinical and policy level. In total, 12 full economic evaluations were included in this review.

The fact that all included articles differed substantially in methodology hampered the attempt to synthesize the information regarding several interventions. The most common design for economic evaluations in epilepsy studies is modelling of patient outcomes and costs. This means that different sources are used in order to gain information, such as data from clinical trials, expert panels, databases etcetera. The advantage is that costs and outcomes can be estimated over a long period of time (often lifetime); the disadvantage is that this estimation holds many assumptions. Strikingly, the advantage of modelling is often not optimized in the studies included in the current review, as the time horizon of $70 \%$ of the articles was only three years or less. The design used less frequently by the studies included in our review, is trialbased economic evaluation. The advantage of this design is the head-to-head comparison of costs and outcomes; the disadvantage being the usually short time horizon and the fact that results cannot be generalized. Only two trial-based economic evaluations are included in our review, unfortunately both with poor methodological quality (e.g. one observational design and one pre-experimental design). Besides the difference between model-based and trial-based economic evaluations, there are many parameters of an economic evaluation which can vary widely from one study to the other. Part of this heterogeneity is unavoidable, because of the multiple choices available when defining parameters of economic evaluations (e.g. designs, outcome measures, patient samples, etcetera). On the other hand, the other part is avoidable, simply by following standard economic research methods. ${ }^{8,10}$ Similar guidelines for many different countries can be found on the website of the International Society for 


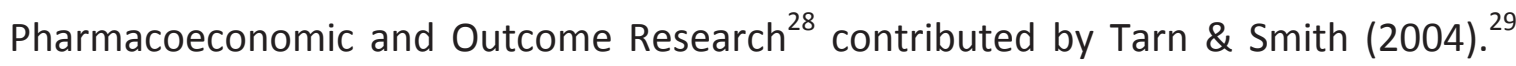
Based on the previously mentioned literature, we distinguish a number of issues that have to be taken into account when conducting an economic evaluation. These are (1) the perspective of the analysis, (2) the nature of economic appraisal and the study design, (3) the outcome measures of costs and consequences and the associated time horizon in which these outcomes can be expected, (4) the discounting of costs and outcomes, (5) and the performance of comprehensive sensitivity analyses. In the present review, we systematically checked the included articles with regard to these five issues and found that they were often poorly described, overlooked, or not taken into account at all. Our findings were consistent with previously published reviews on economic evaluations of epilepsy treatment. A decade ago, Heaney \& Begley (2002) also described the assessment of the economic impact of treatment of epilepsy as challenging. ${ }^{30}$ They describe that the economic assessment is in an early developmental stage and that there is work to be done in the development of generic and specific measures of health states in epilepsy that are appropriate for general use in cost-effectiveness studies. In that same year, Levy (2002) published a methodological review which recommends that epileptologists and health economists should collaborate to achieve much more robust results by applying better methodological guidelines. ${ }^{7}$ A more recently published review by Beghi et al. (2008) describes methodological drawbacks such as virtually absent information, short time horizons, sophisticated models which do not represent clinical practice, use of data from indirect comparisons, and the rare use of QOL measures. ${ }^{31}$ The latter is a huge drawback as this is essential input for economic evaluations. These preferences for health reflect the relative value of time spent in different health states and form the link between information on mortality and on non-fatal health outcomes in summary metrics such as QALYs. Remarkably, in our review almost $70 \%$ of the included articles did use QALYs as an outcome measure. Unfortunately, this also leads to adding another source of heterogeneity because of the many ways of obtaining preferences as described earlier. The most recently published review on economic evaluations in epilepsy treatment concluded that although cost-effectiveness estimates vary between studies, add-on therapy for refractory epilepsy with AEDs appears to be costeffective. $^{32}$ The cost-effectiveness of the drugs does, however, depend on the willingness-to-pay-threshold used.

The inferior quality of the studies was not unique to epilepsy. Previous research in other fields has also shown that sound economic evaluation generally receives little attention in current evaluation studies, in contrast to medical outcome research. ${ }^{33,34}$

\section{Conclusions}

To summarize, this review addresses several important comments to be taken into account when conducting an economic evaluation in the field of epilepsy, in order to improve the methodological quality. In comparison with the review of Levy, ${ }^{7}$ the 
quality of economic evaluations remains suboptimal. A few of the included articles were, however, rather good examples of a sound economic evaluation. ${ }^{20,22}$ We are, therefore, convinced that, when following existing guidelines, it is possible to perform a decent economic evaluation within the field of intractable epilepsy. 


\section{References}

1. Kossoff EH, Zupec-Kania BA, Amark PE, Ballaban-Gil KR, Christina Bergqvist AG, Blackford R, Buchhalter JR, Caraballo RH, Helen Cross J, Dahlin MG, Donner EJ, Klepper J, Jehle RS, Kim HD, Christiana Liu YM, Nation J, Nordli DR, Jr., Pfeifer HH, Rho JM, Stafstrom CE, Thiele EA, Turner Z, Wirrell EC, Wheless JW, Veggiotti P, Vining EP. Optimal clinical management of children receiving the ketogenic diet: recommendations of the International Ketogenic Diet Study Group. Epilepsia 2009; 50:304-17.

2. Tetto A, Manzoni P, Millul A, Beghi E, Garattini L, Tartara A, Avanzini G. The costs of epilepsy in Italy: a prospective cost-of-illness study in referral patients with disease of different severity. Epilepsy Res 2002; 48:207-16.

3. Beghi E, Garattini L, Ricci E, Cornago D, Parazzini F. Direct cost of medical management of epilepsy among adults in Italy: a prospective cost-of-illness study (EPICOS). Epilepsia 2004;45:171-8.

4. Kotsopoulos IA, Evers SM, Ament AJ, Kessels FG, de Krom MC, Twellaar M, Metsemakers JF, Knottnerus AJ. The costs of epilepsy in three different populations of patients with epilepsy. Epilepsy Res 2003;54:131-40.

5. Pugliatti M, Beghi E, Forsgren L, Ekman M, Sobocki P. Estimating the cost of epilepsy in Europe: a review with economic modeling. Epilepsia 2007;48:2224-33.

6. Taylor RS, Sander JW, Taylor RJ, Baker GA. Predictors of health-related quality of life and costs in adults with epilepsy: A systematic review. Epilepsia 2011;52:2168-80

7. Levy P. Economic evaluation of antiepileptic drug therapy: a methodologic review. Epilepsia 2002; 43:550-8.

8. Drummond MF, O'Brien BJ, Sculpher MJ. Methods for the Economic Evaluation of Health Care Programmes. New York: Oxford University Press; 2005.

9. Brazier J, Ratcliffe J, Salomon JA, Tsuchiya A. Measuring and Valuing Health Benefits for Economic Evaluation. New York: Oxford University Press; 2007.

10. Glick HA, Doshi JA, Sonnad SS, Polsky D. Economic Evaluation in Clinical Trials. New York: Oxford University Press; 2007.

11. Maynard A. The design of future cost-benefit studies. Am Heart J 1990;119:761-5.

12. Sassi F, Archard L, McDaid D. Searching literature databases for health care economic evaluations: how systematic can we afford to be? Med Care 2002;40:387-94.

13. Alton V, Eckerlund I, Norlund A. Health economic evaluations: how to find them. Int J Technol Assess Health Care 2006;22:512-7.

14. Soto J. Health economic evaluations using decision analytic modeling. Principles and practicesutilization of a checklist to their development and appraisal. Int J Technol Assess Health Care 2002; 18:94-111.

15. Evers S, Goossens M, de Vet H, van Tulder M, Ament A. Criteria list for assessment of methodological quality of economic evaluations: Consensus on Health Economic Criteria. Int J Technol Assess Health Care 2005;21:240-5.

16. Maltoni S, Messori A. Lifetime cost-utility analysis of patients with refractory epilepsy treated with adjunctive topiramate therapy: cost-effectiveness in refractory epilepsy. Clin Drug Investig 2003; 23:225-32.

17. Lorier JL, Godfroid P, Hart Y, Roberts R. Levetiracetam: an innovative and cost-effective add-on drug for refractory partial epilepsy. Expert Rev Pharmacoecon Outcomes Res 2004;4:143-51.

18. Remak E, Hutton J, Selai CE, Trimble MR, Price MJ. A cost-utility analysis of adjunctive treatment with newer antiepileptic drugs in the UK. Journal of Medical Economics 2004;7:29-40.

19. Blais L, Sheehy O, St-Hilaire JM, Bernier G, Godfroid P, LeLorier JJ. Economic evaluation of levetiracetam as an add-on therapy in patients with refractory epilepsy. Pharmacoeconomics 2005; 23:493-503.

20. Hawkins N, Epstein D, Drummond M, Wilby J, Kainth A, Chadwick D, Sculpher M. Assessing the costeffectiveness of new pharmaceuticals in epilepsy in adults: the results of a probabilistic decision model. Med Decis Making 2005;25:493-510.

21. Knoester PD, Boendermaker AJ, Egberts AC, Hekster YA, Keyser A, Severens JL, Renier WO, Deckers CL. Cost-effectiveness of add-on lamotrigine therapy in clinical practice. Epilepsy Res 2005;67:143-51. 
22. Spackman DE, Yeates A, Rentz AM, Hutton J. The cost effectiveness of zonisamide as adjunctive therapy in adult partial seizure epilepsy. Journal of Medical Economics 2007;10:455-73.

23. Vera-Llonch M, Brandenburg NA, Oster G. Cost-effectiveness of add-on therapy with pregabalin in patients with refractory partial epilepsy. Epilepsia 2008;49:431-7.

24. Suh GH, Lee SK. Economic Evaluation of Add-on Levetiracetam for the Treatment of Refractory Partial Epilepsy in Korea. Psychiatry Investig 2009;6:185-93.

25. Benedict A, Verdian L, Maclaine G. The cost effectiveness of rufinamide in the treatment of LennoxGastaut syndrome in the UK. Pharmacoeconomics 2010;28:185-99.

26. Bolin K, Berggren F, Forsgren L. Lacosamide as treatment of epileptic seizures - cost utility results for Sweden. Acta Neurol Scand 2010;121:406-12.

27. Verdian L, Yi Y. Cost-utility analysis of rufinamide versus topiramate and lamotrigine for the treatment of children with Lennox-Gastaut Syndrome in the United Kingdom. Seizure 2010;19:1-11.

28. Pharmacoeconomic Guidelines Around the World [http://www.ispor.org/peguidelines/index.asp]

29. Tarn TYH, Smith MD. Pharmacoeconomic Guidelines Around the World. Ispor connections 2004;10: 5-15

30. Heaney DC, Begley CE. Economic evaluation of epilepsy treatment: a review of the literature. Epilepsia 43 Suppl 2002;4:10-6.

31. Beghi E, Atzeni L, Garattini L. Economic analysis of newer antiepileptic drugs. CNS Drugs 2008;22:86175.

32. Simoens S. Pharmacoeconomics of anti-epileptic drugs as adjunctive therapy for refractory epilepsy. Expert Rev Pharmacoecon Outcomes Res 2010;10:309-15.

33. Buck AK, Herrmann K, Stargardt T, Dechow T, Krause BJ, Schreyogg J. Economic evaluation of PET and PET/CT in oncology: evidence and methodologic approaches. J Nucl Med Technol 2010;38:6-17.

34. Mistry H. Systematic review of studies of the cost-effectiveness of telemedicine and telecare. Changes in the economic evidence over twenty years. J Telemed Telecare 2012;18:1-6. 


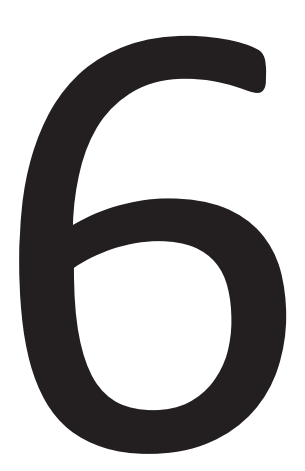

Research into the (Cost-)effectiveness of the ketogenic diet among children and adolescents with intractable epilepsy: design of a randomized controlled trial

RJA de Kinderen, DAJE Lambrechts, D Postulart, AGH Kessels, JGM Hendriksen, AP Aldenkamp, SMAA Evers, HJM Majoie BMC Neurology 2011;11:10 


\section{Abstract}

\section{Background}

Epilepsy is a neurological disorder, characterized by recurrent unprovoked seizures which have a high impact on the individual as well as on society as a whole. In addition to the economic burden, epilepsy imposes a substantial burden on the patients and their surroundings. Patients with uncontrolled epilepsy depend heavily on informal care and on health care professionals. About $30 \%$ of patients suffer from drug-resistant epilepsy. The ketogenic diet can be a treatment of last resort, especially for children. The beneficial effect of the ketogenic diet has been proven, but information is lacking about its cost-effectiveness. In the current study we will evaluate the (cost-)effectiveness of the ketogenic diet in children and adolescents with intractable epilepsy.

\section{Methods/Design}

In a RCT we will compare the ketogenic diet with usual care. Embedded in this RCT will be a trial-based and model-based economic evaluation, looking from a societal perspective at the cost-effectiveness and cost-utility of the ketogenic diet versus usual care. Fifty children and adolescents (aged 1-18) with intractable epilepsy will be screened for eligibility before randomization into the intervention or the usual care group. The primary outcome measure is the proportion of children with a $50 \%$ or more reduction in seizure frequency. Secondary outcomes include seizure severity, side-effects/complaints, neurocognitive, socio-emotional functioning, and quality of life. Costs and productivity losses will be assessed continuously by a prospective diary and a retrospective questionnaire. Measurements will take place during consults at baseline, at 6 weeks and at 4 months after the baseline period, and 3, 6, 9 and 12 months follow-up after the 4 months consult.

\section{Discussion}

The proposed research project will be the first study to provide data about the costeffectiveness of the ketogenic diet for children and adolescents with intractable epilepsy, in comparison with usual care. It is anticipated that positive results in (cost-)effectiveness of the proposed intervention will contribute to the improvement of treatment for epilepsy in children and adolescents and will lead to a smaller burden to society. 


\section{Introduction}

Epilepsy is a brain disorder characterized predominantly by recurrent and unpredictable interruptions of normal brain function. Seizures are transient occurrences of signs and/or symptoms due to such abnormal excessive or synchronous neuronal activities in the brain. ${ }^{1}$ Different parts of the brain can be the site of such discharges. The clinical manifestations of seizures will therefore vary and depend on where in the brain the disturbance first starts and how far it spreads. Transient symptoms can occur, such as loss of awareness or consciousness and disturbances of movement, sensation, mood or mental function. ${ }^{1}$ Furthermore, epilepsy is a major cost-intensive and worldwide health problem. ${ }^{2}$

In 2000, the aggregate burden due to epilepsy was $0.5 \%$ of the total burden of diseases in the world. ${ }^{3}$ In Europe, about 3.4 million people suffer from epilepsy. ${ }^{4}$ The annual total costs of epilepsy in Europe are $€ 15.5$ billion. ${ }^{5}$ In the Netherlands, direct medical costs were $€ 221$ million in $2005 .^{6}$ In addition to the economic burden, epilepsy imposes a substantial burden on the patients themselves and their surroundings.

In the Netherlands, the point prevalence of epilepsy in children and adolescents between $0-18$ years is 4.77 boys, and 4.55 girls per 1,000 persons. The yearly incidence of epilepsy in the Netherlands for children and adolescents between $0-18$ years is 0.58 per 1,000 boys and 0.53 per 1,000 girls. $^{7}$ Although epilepsy is treatable with antiepileptic drugs in the majority of cases, about $30 \%$ of patients suffer from drug-resistant epilepsy. ${ }^{8}$ Patients with uncontrolled epilepsy heavily depend on informal care (family and friends) and on health care professionals (neurologists, social workers, psychologists etc.). Complications due to intractable epilepsy result in frequent hospitalizations and many of these patients are institutionalized. Patients with drug-resistant epilepsy can potentially benefit from a ketogenic diet. The ketogenic diet is a high-fat, low carbohydrate, normocaloric diet that mimics the metabolic state of fasting. During a prolonged fast, body energy requirements are met by lipolysis and ß-oxidation of fatty acids rather than by the breakdown of carbohydrates. The ketogenic diet maintains an anabolic nutritional state in a metabolic situation of fasting. Ketone bodies may produce an anticonvulsant effect, presumably due to changes in cerebral energy metabolism, in cell properties decreasing excitability, in neurotransmitter function, in circulating factors acting as neuromodulators and in the brain's extracellular milieu. ${ }^{9}$ The ketogenic diet is generally used for a period of up to 3 years. Seizure control benefits are typically seen within 1-3 months of starting the diet. The international study group reports that the diet should be utilized for at least $31 / 2$ months before deciding to discontinue it. ${ }^{10}$ The diet can be discontinued earlier if seizures worsen beyond expectations or if adverse effects cannot be corrected. ${ }^{11}$ Medications are tapered once the efficacy of the diet has been established (usually within 3-6 months of diet initiation). The diet is gradually tapered off by lowering its fat content and increasing the carbohydrate and 
protein portion of the diet until ketosis is eliminated. Tapering starts after 2 years of treatment, or earlier in case of intolerable side-effects, or in case of ineffectiveness. Currently the scientific and clinical attention paid to the role of the ketogenic diet is negligible. This means that a ketogenic diet is often overlooked and underutilized as a treatment option for children with intractable epilepsy. An important reason for this is that relatively few children and their parents can comply with the stringent diet. Therefore, physicians are often reluctant to initiate the diet. To offer more children the opportunity to benefit from a ketogenic diet, we suggest that the diet should be initiated and monitored under strictly controlled circumstances in order to maximize compliance.

The beneficial effect of a ketogenic diet has been studied in multiple observational studies, ${ }^{12-20}$ reviews ${ }^{21-24}$ and in one randomized controlled trial. ${ }^{25}$ Nevertheless, there is a lack of information about the cost-effectiveness of the ketogenic diet, and consequently health authorities have not been convinced of its usefulness.

The aims of our prospective study are to provide more evidence about the effectiveness and to investigate the cost-effectiveness of treatment with a ketogenic diet KD in comparison with usual care in children and adolescents with drug-resistant epilepsy who are not eligible for epilepsy surgery.

\section{Methods/Design}

The design and methods of a randomized controlled trial (RCT) evaluating the (cost-)effectiveness of a ketogenic diet in comparison with usual care in children and adolescents with intractable epilepsy are described in this paper. This study has been approved by the ethics committee of the Academic Medical Centre Utrecht, the Netherlands.

\section{Research question}

We defined the following research questions:

\section{Effect evaluation}

What are the effects of the ketogenic diet in comparison with usual care with respect to changes in seizure frequency and seizure severity, side-effects/complaints, neurocognitive, socio-emotional functioning, and quality of life?

\section{Economic Evaluation}

The economic evaluation will consist of a trial-based and a model-based economic evaluation study. 
Ila) Trial-based economic evaluation

From a social perspective, is the ketogenic diet, in comparison with usual care, preferable in terms of costs, effects and utilities?

What is the incremental cost-effectiveness ratio (ICER) of the ketogenic diet in comparison with usual care?

IIb) Model-based economic evaluation

In comparison with usual care, is the ketogenic diet preferable in terms of costs and utilities during the remaining life expectancy of the study population?

\section{Design}

The proposed study is a prospective RCT. The subjects will be randomized to either the ketogenic diet or to usual care. The trial flow of the proposed subject enrolment and randomization procedures are shown graphically in Figure 6.1. Embedded in this RCT will be a short-term trial-based economic evaluation and a long-term modelling study to investigate the long-term cost-effectiveness and cost-utility of the ketogenic diet versus usual care.

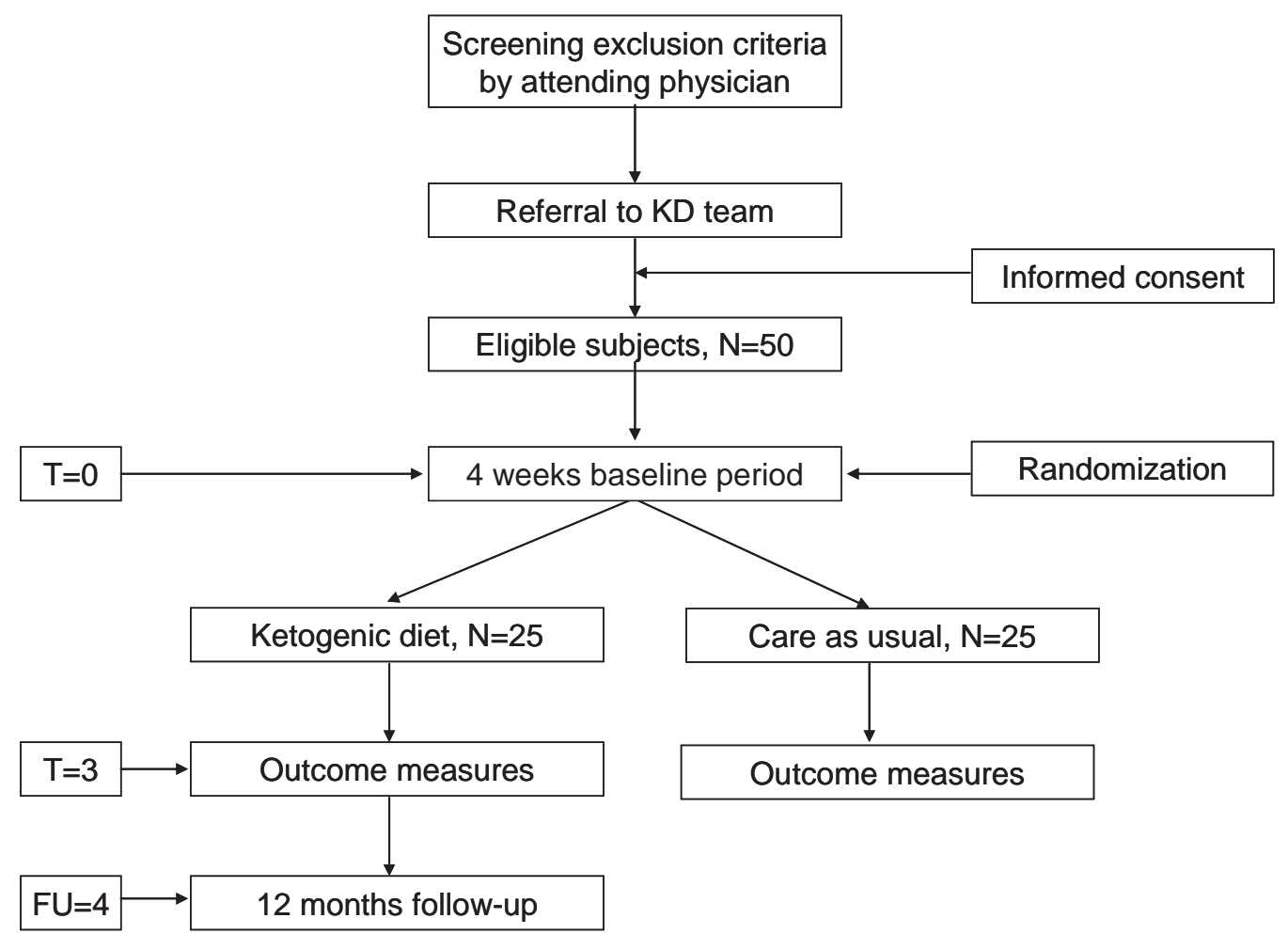

Figure 6.1 Flowchart of the study. 


\section{Participants}

The patient population consists of children and adolescents with drug-resistant epilepsy. Patients are eligible to participate if they meet the following criteria: age between 1 and 18 years old; having uncontrolled seizures; not eligible for epilepsy surgery; no fatty acid oxidation disorders and related diseases; no diabetes and hyperinsulinism; no prolonged QT-time syndrome; no hypercholesterolemia or hypertriglyceridemia; no severe liver, kidney or pancreas diseases; no renal tubular acidosis; no severe behavioral disorder; no malnutrition; no treatment with topiramate or acetazolamide and no positive family history or other risk factors for kidney stones or acidosis.

\section{Recruitment}

Potential participants are screened for exclusion criteria by their own attending pediatrician, neurologist or pediatric neurologist. Children who are eligible to be treated with a ketogenic diet are subsequently referred to the multidisciplinary ketogenic diet team at the epilepsy center Kempenhaeghe in the Netherlands. Eligible children and their parents will first receive information by telephone before getting an invitation for an information visit. During the information visit eligibility is checked by the multidisciplinary team. If they are interested, parents, adolescents and/or children can find information about the study on the website http://www.ketogeenmenu.nl. Parents and potential candidates who are considering participating in the study will have at least one week to decide, before signing informed consent.

\section{Sample size}

A $50 \%$ reduction in seizure frequency is considered clinically relevant and is defined as success. Assuming a minimum detectable difference in success rate of $35 \%$ between the ketogenic diet group and usual care, and assuming that alpha $=5 \%$ and power $=80 \%$, we need 22 children for each group. Taking dropouts into account, we need 50 children in total. A drop-out is defined as a child who drops out of the study before the first consultation with the neurologist; this is scheduled 6 weeks after initiating the KD or 6 weeks after randomization to usual care.

\section{Randomization and procedure}

Randomization will occur halfway through the 4 week baseline period. We will use a software package (ALEA) to support the online patient registration and randomization which will be based on the minimization method. Patients will be stratified according to age (1-6 years, 7-12 years, 13-18 years), having a PEG/ tube or not, and whether the child is living at a residential center or attends the epilepsy center as an outpatient 
while she/he lives at home. In view of the nature of the treatments, blinding of the patients and researchers is not possible.

\section{Intervention}

\section{Ketogenic diet}

Patients assigned to the ketogenic diet group will be hospitalized in the tertiary epilepsy center for one week. During this week the ketogenic diet is introduced by a dietician. The antiepileptic drugs the children and adolescents use at the time of inclusion in the study will be continued without changes during the study (except when medically indicated). The initial calorie prescription for the ketogenic diet is based on an average between the pre-diet intake and the recommendations for energy requirements, taking into account current and previous weight and height, recommended caloric requirements and levels of physical activity. The dietician will decide together with the parents whether the classical diet or the medium-chain triglyceride (MCT) diet is introduced. Sometimes the MCT diet is not possible and the classical ketogenic diet (3:1 or 4:1 ratio) is advised. Children with a PEG/tube feed are also treated with the ketogenic diet. The diet is then adjusted to a fluid version. It is possible to decrease the titration of antiepileptic drugs in responders from six months after the initiation of the ketogenic diet.

\section{Usual care}

Patients randomized to usual care will continue to take their antiepileptic drugs and no changes will be made to the antiepileptic drugs treatment. Since a ketogenic diet is a treatment of last resort, the children in the usual care group will also receive a ketogenic diet after a delay of four months. The controls will be treated and monitored according to good clinical practice; however, this is not part of our proposed study.

\section{Timeline of the study}

The timeline of the study is shown in Figure 6.2. Patients in the intervention group will visit the multidisciplinary team (nurse practitioner, dietician, paediatrician and neurologist) at time points T0 (at baseline), T1 (during admission), T2 (6 weeks after admission), T3 (4 months after admission), FU1 (3 months follow-up), FU2 (6 months follow-up), FU3 (9 months follow-up), and FU4 (12 months follow-up). At T0, T3, and FU4 a neurocognitive assessment will take place. An overview of measurements per time point is shown in Table 1. Patients in the intervention group will be asked to follow the diet during the complete study period and during follow-up, 16 months in total. 
Patients in the usual care group will visit the multidisciplinary team (nurse practitioner, pediatrician and neurologist) at time points T0, T2, and T3 and will have a neurocognitive assessment at T0 and T3. In case of drop-out, a visit with the multidisciplinary team and a neurocognitive assessment will take place.

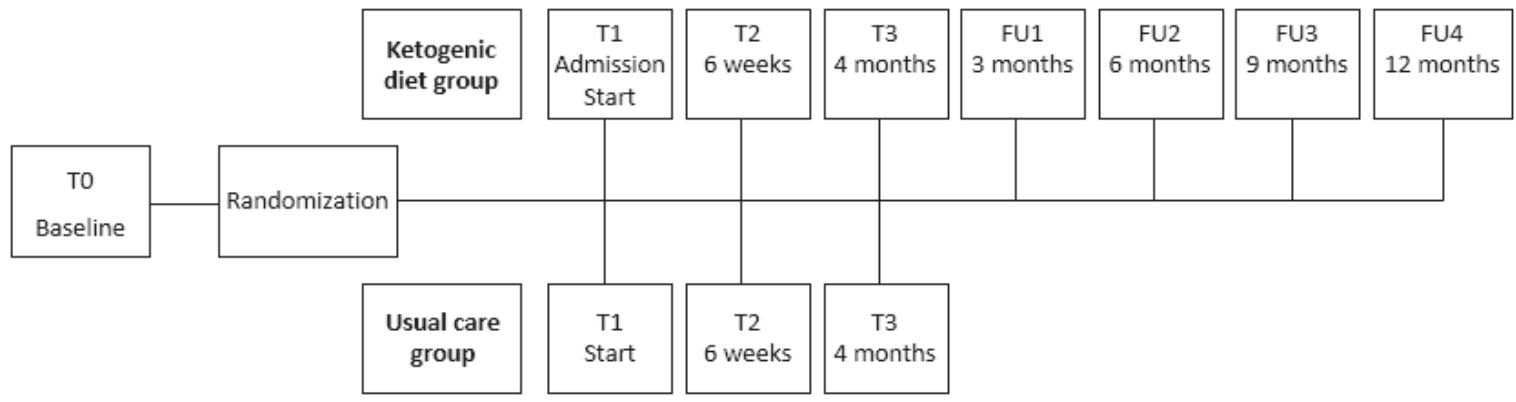

Figure 6.2 Timeline of the study.

T0: baseline; T1: admission; T2: 6 weeks after baseline period; T3: 4 months after baseline period; FU1: 3 months follow-up; FU2: 6 months follow-up; FU3: 9 months follow-up; FU12: 12 months follow-up.

\section{Effect evaluation}

Several instruments will be used to assess the effects of the ketogenic diet on the primary outcome (seizure frequency) and on secondary outcomes (seizure severity, side-effects, neurocognitive assessment and quality of life).

\section{Primary outcome}

Seizure frequency

A seizure calendar is used to record seizures. Types of seizures are described and labelled in accordance with ILAE classification. The number and type of seizures are recorded on the dates they occur.

\section{Secondary outcome}

Seizure severity

The National Hospital Seizure severity scale $(\mathrm{NHS} 3)^{26}$ is a structured interview in which the clinician rater assigns a score to seizure severity based on interference with patient function. Information is gathered from the patient and witnesses, if available. Eight questions are asked about: tonic-clonic movements, falling, injury, incontinence, altered consciousness, postictal impairment, and disruptive automatisms.

\section{Side-effects}

Subjective complaints are assessed using the SIDAED (Side-effects of Antiepileptic Drug), a list of 46 items with possible antiepileptic drug-related complaints. The items 
included in the SIDAED form 10 categories: general Central Nervous System (CNS), behavior (increased irritability), depressive symptoms, changes in cognitive function, motor problems and coordination, visual complaints, headache, cosmetic and dermatological complaints, gastrointestinal complaints, and sexuality and menses. For each item the scale (no problem, mild, moderate, or serious problem). In addition, the duration of the complaints is scored (for a few weeks, for months or for a half year or longer).

Table 6.1 Overview of measurements per time point.

\begin{tabular}{|c|c|c|c|c|c|c|c|c|}
\hline Measurement & TO & T1* & T2 & T3 & FU1* & FU2* & FU3* & FU4* \\
\hline Demographic characteristics & $x$ & & & & & & & \\
\hline Clinical measurement & $x$ & $x$ & $x$ & $x$ & $x$ & $x$ & $x$ & $x$ \\
\hline National Hospital Seizure severity scale & $x$ & $x$ & $x$ & $x$ & $x$ & $x$ & $x$ & $x$ \\
\hline Side-effects of Antiepileptic Drug & $x$ & $x$ & $x$ & $x$ & $x$ & $x$ & $x$ & $x$ \\
\hline Credibility And Expectancy Questionnaire & $x$ & & & & & & & \\
\hline Peabody Picture Vocabulary test & $x$ & & & $x$ & & & & $x$ \\
\hline Beery Developmental Visual-Motor Integration test & $x$ & & & $x$ & & & & $x$ \\
\hline FEPSY computerized test & $x$ & & & $x$ & & & & $x$ \\
\hline Kaufman Assessment Battery & $x$ & & & $x$ & & & & $x$ \\
\hline Actigraphy & $x$ & & & $x$ & & & & $x$ \\
\hline SEV Questionnaire & $x$ & & & $x$ & & & & $x$ \\
\hline SDQ Questionnaire & $x$ & & & $x$ & & & & $x$ \\
\hline POMS Questionnaire & $x$ & & & $x$ & & & & $x$ \\
\hline The Personal Adjustment and Role Skills Scale & $x$ & & & $x$ & & & & $x$ \\
\hline The Hague Restrictions in Childhood Epilepsy Scales & $x$ & & & $x$ & & & & $x$ \\
\hline EuroQol & $x$ & & & $x$ & & & & $x$ \\
\hline TAPQOL or TACQOL & $x$ & & & $x$ & & & & $x$ \\
\hline Blood & $x$ & & $x$ & $x$ & $x$ & $x$ & $x$ & $x$ \\
\hline Urine & $x$ & & $x$ & $x$ & $x$ & $x$ & $x$ & $x$ \\
\hline Keton bodies measurement & $x$ & $\mathrm{x}$ & $x$ & $x$ & $x$ & $x$ & $x$ & $x$ \\
\hline ECG & $x$ & & & $x$ & $x$ & $x$ & $x$ & $x$ \\
\hline
\end{tabular}

T0: baseline; T1: admission; T2: 6 weeks after baseline period; T3: 4 months after baseline period; FU1: 3 months follow-up; FU2: 6 months follow-up; FU3: 9 months follow-up; FU12: 12 months follow-up; * intervention group only.

\section{Neurocognitive assessment}

Peabody Picture Vocabulary test

The Peabody Picture Vocabulary test (PPVT-III-NL: Dutch version) is an individually administered measure of receptive vocabulary. ${ }^{27}$ The test can be administered beginning at the age of $21 / 2$ years. The child listens to the examiner's stimulus word and must choose the picture that best describes the word from a 4-picture multiple choice array. The score on this test can be interpreted as an achievement test of the child's vocabulary that does not require a verbal response and as a screening measure of verbal ability. There is supportive data for the validity of the PPVT-III as a measure for global cognitive intelligence. 
Beery Developmental Visual-Motor Integration test

The Beery Developmental test of Visual-Motor Integration (Beery-VMI) is a widely used assessment of psychomotor development. It measures problems with visual perception, motor coordination, and visual motor integration. The 30-item test can be administered from the age of 2 years. The Beery VMI was standardized on a national US sample of 1,737 individuals age 2 to 18 years (2010) and 1,021 adults ages 19-100 (2006), and has proven reliability and validity. ${ }^{28}$

\section{FEPSY computerized test}

The FEPSY is a neurocognitive computerized test battery consisting of different subtests. For this study we use the simple auditory and visual reaction times, in which the stimulus exposure endures until a push-button (space-bar) response is given. ${ }^{29}$ Simple reaction tests give information on alertness functions and on the speed at which the information processing system is activated. We also use the binary choice reaction task in which a combination of accuracy and speed of responses are measured. This test measures the speed of central information processing. Finally, we use the finger tapping task in which motor activation and fluency is measured. In general, these tests can be administered from the age of 6 .

\section{Kaufman Assessment Battery}

The Kaufman Assessment Battery for Children II (K-ABC II) is used to investigate the level of information processing capacities of children beginning at the age of $21 / 2$ years. ${ }^{30}$ Two subtests will be administered: (1) Number recall, which is a measure of sequential processing and short term memory. The examiner says a series of numbers and the child repeats them. Digit span forward is considered a measure of efficiency of attention or "freedom from distractibility" and (2) conceptual thinking, which is a measure of nonverbal reasoning skills. The child looks at pictures and determines the one that does not belong.

\section{Actigraphy}

During the administration of the PPVT-III and the K-ABCII test, an actigraphic device (Actiwatch ${ }^{\circledR}$ model AW4: Cambridge Neurotechnology Ltd., United Kingdom) will be used to assess motor activity as a measure of alertness. As some of the children in the design are not testable with the described cognitive tests we decided to use an assessment of motor activity in a standardized situation. A wrist watch will be used that translates activity into movement of the watch hand. Registration takes place in a standardized way during the first 10 minutes of the Peabody Picture Vocabulary test and the first 5 minutes of the K-ABC-II test. When patients are not eligible to perform the neurocognitive assessment, the wrist watch will be used for 15 minutes during the visit at the nurse practitioner's office while watching a DVD with songs and pictures. 


\section{SEV Questionnaire}

The SEV questionnaire (Sociaal Emotionele Vragenlijst: Social Emotional Questionnaire) is a DSM-IV oriented questionnaire which is used to assess four domains of behavioral and social emotional dysfunction. These domains are: Attention Deficit Hyperactivity Disorders, Oppositional Defiant behavior and Conduct Disorders, Anxiety and Depression, and Autism spectrum disorders. The questionnaire consists of 72 items describing problem behaviour. ${ }^{31}$

\section{SDQ Questionnaire}

The Strengths and Difficulties Questionnaire (SDQ) is a brief behavioral screening questionnaire that provides balanced coverage of children and young people's behaviors, emotions, and relationships. ${ }^{32}$ The SDQ is applicable to children beginning at the age of 4 and asks about 25 attributes that are divided into five relevant dimensions: namely conduct problems, emotional symptoms, hyperactivity, peer relationships, and pro-social behavior.

\section{POMS Questionnaire}

The Profile of Mood States (POMS) has been developed to identify and assess seven transient, fluctuating affective mood states: Tension-Anxiety, Depression-Dejection, Anger-Hostility, Vigor-Activity, Fatigue-Inertia, Friendliness, and ConfusionBewilderment. ${ }^{33}$ The questionnaire contains 65 items. All items describe an emotional state which can be rated on a 5-point scale ranging from "not at all" to "extremely".

The Personal Adjustment and Role Skills Scale (PARS)

The Personal Adjustment and Role Skills Scale, 3rd edition (PARS-III) was specifically developed to measure psychosocial adjustment in children with chronic physical illness. ${ }^{34}$ This instrument is a brief parent-completed index of youth psychosocial adjustment. The instrument yields six factor-derived psychosocial subscales: namely peer relations, dependency, hostility, productivity, anxiety/depression, and withdrawal. The PARS-III is a reliable and valid index of youth psychosocial adjustment and can be used for both clinical screening and research purposes. ${ }^{35}$

The Hague Restrictions in Childhood Epilepsy Scales (HARCES)

The HARCES is a questionnaire consisting of 11 items used to assess impairments in daily functioning that are related to epilepsy, e.g. how much influence does the epilepsy have on your child's freedom of functioning in the house? Items are answered on a 4-point scale ranging from "no impairment" to "very much impairment". 36

Credibility And Expectancy questionnaire (CEQ)

The Credibility and Expectancy Questionnaire (CEQ) ${ }^{37}$ is a multidimensional selfreported instrument of 6 items, rated on a 9- or 10-point Likert-scale, meant to 
measure the expectancy and credibility a person has about the therapy he or she will receive. Its sound psychometric properties have been proven. We will measure the credibility and expectancy for the treatment of both the children and their parents.

\section{Generic quality of life}

\section{EuroQol}

Living with uncontrolled seizures has a negative impact on the quality of life of the child and his/her parents. Therefore, we will measure generic quality of life of both the children and their parents. For this purpose we will use the EuroQol instrument for children of 12 years and older developed by the EuroQol group. ${ }^{38}$ For younger children, we will use a version of the EuroQol developed for children (EuroQol-Youth) or proxies. In accordance with the regular EuroQol, the EuroQol-Youth is comprised of the following 5 dimensions: mobility, self-care, usual activities, pain/discomfort and anxiety/depression. Each dimension has 3 levels: no problems, some problems and severe problems, thus defining $243\left(3^{5}\right)$ possible health states. 'Unconscious' and 'dead' have been added to these states, resulting in a total of 245 states. Furthermore, the EQ-5D consists of a visual analogue scale (VAS) ranging from zero (worst imaginable health state) to 100 (best imaginable health state).

\section{TAPQOL or TACQOL}

QoL will also be assessed using the TAPQOL (TNO-AZL Preschool Children's Quality of Life) for children aged between 1-5 years ${ }^{39}$ and the TACQOL (TNO-AZL Children's Quality of life) for children aged between 6-16 years. ${ }^{40}$ There are 12 dimensions in the TAPQOL: lungs, stomach, skin, sleep, appetite, aggressive behavior, positive emotions, fear, vitality, social behavior, motor functioning and communication. All dimensions are scored on a scale from $0-100$. The TACQOL is comprised of 7 dimensions: bodily complaints, independence, motor functioning, cognition, social functioning, positive and negative emotions. The scores of the first 5 dimensions vary from 0-32 and the scores of the last two dimensions from 0-16.

\section{Economic evaluation}

The economic evaluation will be performed from the societal perspective, which implies that all relevant costs and effects will be taken into account. The economic evaluation compares costs and effects of the ketogenic diet in comparison with usual care. A Cost-Effectiveness Analysis (CEA) and a Cost-Utility Analysis (CUA) will be performed. Outcomes of interest for the CEA and the CUA will be the reduction of seizures and improvement in the Quality Adjusted Life Years (QALYs) respectively. The QALY is a measure of disease burden, including both the quality (utilities) and the quantity of life lived. Total societal costs will be calculated based on the Dutch guidelines for cost calculations in health care. We distinguish four cost categories: 
intervention costs, health care sector costs, costs for the patient and family, and productivity costs. For this study, three instruments are used to measure the costs and utilities.

\section{Prospective cost diary}

The diary is used to identify all relevant cost aspects with respect to health care sector costs and patient and family costs. ${ }^{41}$ Each diary covers a period of four weeks and will be filled out during the 4 week baseline period and during the 4 month study period. The intervention group will also fill out the diary during the 12 months of follow-up. Total costs will be estimated using a bottom-up approach, where information on each element of service used will be multiplied by an appropriate standardized unit cost and be summed to provide an overall total cost. For the cost valuation, standardized cost prices from the Dutch manual for cost analysis in health care research will be used. $^{42}$

\section{Productivity losses}

A retrospective questionnaire and the patient modules of the PROductivity and DISease Questionnaire (PRODISQ) ${ }^{43}$ will be used to measure production losses of the patient's parents. Productivity costs will be calculated by means of the friction cost method, based on a mean added value of the Dutch working population. This method takes into account production losses confined to the period needed to replace a sick employee.

\section{Utilities (EQ-5D)}

Both generic quality of life, as well as utilities, will be derived by means of the EuroQol, as mentioned before. Utility values will be calculated for all health states of the EuroQol, using preferences from the UK general population value set which provides an algorithm as a series of decrements from 1, the value of full health. Values of the UK population for the scoring function of the health states described by the EQ5D were measured with the Time Trade Off (TTO) technique (see Torrance, 1976) on a random sample of approximately 3000 members of the adult population of the UK. ${ }^{44}$ The utility values derived from the Dolan algorithm will be used to compute Quality Adjusted Life Years (QALYs). However, to overcome the tariff's differences between countries, the Dutch tariff is also used to calculate utilities in the sensitivity analysis. Lamers, ${ }^{45}$ calculated by means of the TTO method a Dutch scoring function for the health states described by the EQ-5D, based on a Dutch population sample of 300 people. 


\section{Analyses}

\section{I) Effect evaluation}

Our primary (base-case) analyses will be performed according to the intention-totreat principle, including data from all participants regardless of whether they received the intervention or not. For the analyses we will use SPSS statistical software. Respondents for whom at least $75 \%$ of the data per measurement instrument are available will be included in the analysis. Missing data on an item level will be handled using SPSS missing value regression analysis. Completely missing measurements will be handled using multiple imputation. Between-group differences in proportions (dichotomous variables) will be tested using the Chi-square test and between-group differences in means (continuous variables) will be tested using Student's t-test for independent samples. In addition, a multivariate regression analysis will be performed with the covariates of sex, age, severity of disease, duration of disease etc. The accuracy of the findings will be expressed in terms of $95 \%$ confidence intervals.

\section{Ila) Trial-based economic evaluation}

A baseline analysis will be performed to examine the comparability of groups at baseline for both costs and outcomes. If necessary, methods will be applied to control for differences in baseline. ${ }^{46} \mathrm{~A}$ Kolmogorov-Smirnov test will be performed to investigate whether data are distributed normally. Despite the usual skewness in the distribution of costs, the arithmetic means will generally be considered the most appropriate measures for describing cost data. ${ }^{47,48}$ Therefore, arithmetic means (and standard deviations) will be presented. In case the cost data are skewed, nonparametric bootstrapping will be used to test for statistical differences in costs between the intervention and usual care. The bootstrap replications will be used to calculate $95 \%$ confidence intervals around the costs $(95 \% \mathrm{Cl})$, based on the 2.5 th and 97.5th percentiles. If cost data are distributed normally, t-tests will be used. The incremental cost effectiveness ratio (ICER) will be determined on the basis of the incremental costs and effects of the ketogenic diet in comparison with a waiting-list group. The cost-effectiveness ratio will be stated in terms of costs per outcome rate (decrease in seizure frequency and severity), and the cost-utility ratio will focus on the net cost per utility gained.

The robustness of the ICER will be checked by non-parametric bootstrapping. Bootstrap simulations will also be conducted in order to quantify the uncertainty around the ICER, yielding information about the joint distribution of cost and effect differences. The bootstrapped cost-effectiveness ratios will be plotted subsequently in a cost effectiveness plane. The choice of treatment depends on the maximum amount of money that society is prepared to pay for a gain in effectiveness, which is called the ceiling ratio. Therefore, the bootstrapped ICERs will also be depicted in a costeffectiveness acceptability curve showing the probability that the ketogenic diet is 
cost-effective using a range of ceiling ratios. In addition, to demonstrate the robustness of our base-case findings, multi-way sensitivity analyses will be performed. $^{49}$

\section{IIb) Model-based economic evaluation}

The time horizon of the Cost Utility Analysis (CUA) will be extrapolated towards the remaining life expectancy of the study population. The CUA is of major importance since the impact of a ketogenic diet on seizure reduction, costs, and QoL reaches beyond the 4-month study period of the clinical study. A Markov Monte Carlo decision analytic model will be developed to calculate lifetime incremental costs and incremental QALYs of treatment with a ketogenic diet in comparison with treatment with antiepileptic drugs only. Markov models assume that a patient is always in one of a finite number of health states. All events are represented as transitions from one state to another. A first-order Monte Carlo evaluation of a Markov model determines the prognoses of a large number of individual patients. The time horizon of the analysis is divided into cycles. Each patient begins in an initial state. During each cycle, the patient may make a transition from one state to another according to the laws of chance, as dictated by the transition probabilities. After the first patient has completed the simulation, another patient begins in the initial state and a new simulation is performed. This process is repeated a very large number of times, and each simulation generates a quality adjusted survival time and costs. Monte Carlo analysis, as opposed to a Markov cohort model without memory, offers the possibility to flag subjects in order to track their characteristics and disease histories; this is a very flexible approach to modelling variability within a population. The model will combine the results of the clinical study and data from the medical literature. In the modelling study we will also perform probabilistic sensitivity analysis to test parameter uncertainty and to construct cost-effectiveness acceptability curves. Future costs and effects will be discounted according to the Dutch guidelines for cost calculations in health care. ${ }^{42}$

\section{Discussion}

Our design is aimed at assessing the effectiveness and cost-effectiveness of a ketogenic diet among children and adolescents with intractable epilepsy. It is a prospective RCT comparing the ketogenic diet with usual care.

The ketogenic diet can be a treatment of last resort for patients with intractable epilepsy. This alternative therapy is used only in the minority of children who could potentially benefit from it. In order to optimize therapy for children with uncontrolled seizures, the ketogenic diet should be prescribed for more children with intractable epilepsy. 


\section{Limitations and complications}

Compliance with a ketogenic diet is difficult due to its restrictive nature. Unfortunately, non-compliance limits the intended effect and increases the costs to society, resulting in a less favorable cost-effectiveness ratio. In order to overcome this problem, the children and adolescents with uncontrolled epilepsy who are on the ketogenic diet will be monitored according to a strict standardized protocol. Therefore it is likely that the efficiency of patient care will improve. However, a formal assessment of the diet's cost-effectiveness has not yet been performed and is the focus of the present proposal.

\section{Conclusion}

Growing up with seizures affects the child's personality and cognitive development and interferes with many aspects of everyday life, including learning at school, leisure and occupational activities. Epilepsy has been shown to have a high impact on quality of life when children have intractable seizures and additional disabilities.

This study will provide information about the cost-effectiveness of the ketogenic diet, its effects on clinical outcomes, on neurocognitive functioning and on quality of life. Our study is the first study assessing the cost-effectiveness of a ketogenic diet. 


\section{References}

1. Fisher RS, van Emde Boas W, Blume W, Elger C, Genton P, Lee P, Engel J Jr. Epileptic seizures and epilepsy: definitions proposed by the International League Against Epilepsy (ILAE) and the International Bureau for Epilepsy (IBE). Epilepsia. 2005;46:470-2.

2. Strzelczyk A1, Reese JP, Dodel R, Hamer HM. Cost of epilepsy: a systematic review. Pharmacoeconomics. 2008;26:463-76.

3. Leonardi M, Ustun TB. The global burden of epilepsy. Epilepsia. 2002;43(Suppl 6):21-5.

4. Forsgren L, Beghi E, Oun A, Sillanpää M. The epidemiology of epilepsy in Europe - a systematic review. Eur J Neurol. 2005;12:245-53.

5. Pugliatti M, Beghi E, Forsgren L, Ekman M, Sobocki P. Estimating the cost of epilepsy in Europe: a review with economic modeling. Epilepsia. 2007;48:2224-33.

6. Poos MJJC, Smit JM, Groen J, Kommer GJ, SLobbe LCJ: Kosten van ziekten in Nederland 2005. RIVM 2008.

7. Hoeymans N, Melse JM, Schoenmaker CG: Gezondheid en determinanten. Deelrapport van de Volksgezondheid Toekomst Verkenning 2010 Van gezond naar beter. RIVM-rapport nr. 270061006 2010.

8. Kossoff EH, Zupec-Kania BA, Amark PE, Ballaban-Gil KR, Christina Bergqvist AG, Blackford R, Buchhalter JR, Caraballo RH, Helen Cross J, Dahlin MG, Donner EJ, Klepper J, Jehle RS, Kim HD, Christiana Liu YM, Nation J, Nordli DR Jr, Pfeifer HH, Rho JM, Stafstrom CE, Thiele EA, Turner Z, Wirrell EC, Wheless JW, Veggiotti P, Vining EP; Charlie Foundation, Practice Committee of the Child Neurology Society; Practice Committee of the Child Neurology Society; International Ketogenic Diet Study Group. Optimal clinical management of children receiving the ketogenic diet: recommendations of the International Ketogenic Diet Study Group. Epilepsia. 2009;50:304-17.

9. Freeman J, Veggiotti P, Lanzi G, Tagliabue A, Perucca E; Institute of Neurology IRCCS C. Mondino Foundation. The ketogenic diet: from molecular mechanisms to clinical effects. Epilepsy Res. 2006;68:145-80.

10. Kossoff EH, McGrogan JR, Bluml RM, Pillas DJ, Rubenstein JE, Vining EP. A modified Atkins diet is effective for the treatment of intractable pediatric epilepsy. Epilepsia. 2006;47:421-4.

11. Zupec-Kania BA, Spellman E. An overview of the ketogenic diet for pediatric epilepsy. Nutr Clin Pract. 2008;23:589-96.

12. Caraballo RH, Cersósimo RO, Sakr D, Cresta A, Escobal N, Fejerman N. Ketogenic diet in patients with Dravet syndrome. Epilepsia. 2005;46:1539-44.

13. Coppola G, Veggiotti P, Cusmai R, Bertoli S, Cardinali S, Dionisi-Vici C, Elia M, Lispi ML, Sarnelli C, Tagliabue A, Toraldo C, Pascotto A. The ketogenic diet in children, adolescents and young adults with refractory epilepsy: an Italian multicentric experience. Epilepsy Res. 2002;48:221-7.

14. Freeman JM, Vining EP, Pillas DJ, Pyzik PL, Casey JC, Kelly LM. The efficacy of the ketogenic diet-1998: a prospective evaluation of intervention in 150 children. Pediatrics. 1998;102:1358-63.

15. Kang HC, Kim YJ, Kim DW, Kim HD. Efficacy and safety of the ketogenic diet for intractable childhood epilepsy: Korean multicentric experience. Epilepsia. 2005;46:272-9.

16. Kankirawatana $\mathrm{P}$, Jirapinyo $\mathrm{P}$, Kankirawatana $\mathrm{S}$, Wongarn $\mathrm{R}$, Thamanasiri N. Ketogenic diet: an alternative treatment for refractory epilepsy in children. J Med Assoc Thai. 2001;84:1027-32.

17. Kossoff EH, Pyzik PL, McGrogan JR, Vining EP, Freeman JM. Efficacy of the ketogenic diet for infantile spasms. Pediatrics. 2002;109:780-3.

18. Kossoff EH, Thiele EA, Pfeifer HH, McGrogan JR, Freeman JM. Tuberous sclerosis complex and the ketogenic diet. Epilepsia. 2005;46:1684-6.

19. Maydell BV, Wyllie E, Akhtar N, Kotagal P, Powaski K, Cook K, Weinstock A, Rothner AD. Efficacy of the ketogenic diet in focal versus generalized seizures. Pediatr Neurol. 2001;25:208-12.

20. Vining EP, Freeman JM, Ballaban-Gil K, Camfield CS, Camfield PR, Holmes GL, Shinnar S, Shuman R, Trevathan E, Wheless JW. A multicenter study of the efficacy of the ketogenic diet. Arch Neurol. 1998;55:1433-7.

21. Keene DL. A systematic review of the use of the ketogenic diet in childhood epilepsy. Pediatr Neurol. 2006;35:1-5. 
22. Lefevre F, Aronson N. Ketogenic diet for the treatment of refractory epilepsy in children: A systematic review of efficacy. Pediatrics. 2000;105:E46.

23. Levy R, Cooper P. Ketogenic diet for epilepsy. Cochrane Database Syst Rev 2003;3: CD001903.

24. Sinha SR, Kossoff EH. The ketogenic diet. Neurologist. 2005;11:161-70.

25. Neal EG, Chaffe H, Schwartz RH, Lawson MS, Edwards N, Fitzsimmons G, Whitney A, Cross JH. The ketogenic diet for the treatment of childhood epilepsy: a randomised controlled trial. Lancet Neurol. 2008;7:500-6.

26. O'Donoghue MF, Duncan JS, Sander JW. The National Hospital Seizure Severity Scale: a further development of the Chalfont Seizure Severity Scale. Epilepsia. 1996;37:563-71.

27. Dunn LM, Dunn LM. Peabody Picture Vocabulary Test-III-NL, Nederlandse versie door Liesbeth Schlichting. Harcourt Assessment B.V., Amsterdam; 2005.

28. Beery K. The Beery-Buktenica Developmental Test of Visual-Motor Integration: VMI with Supplemental Developmental Test of Visual Perception and Motor Coordination: Administration, Scoring and Teaching Manual. Parsippany, NJ: Modern Curriculum Press; 1997.

29. Alpherts WC, Aldenkamp AP. Computerized neuropsychological assessment of cognitive functioning in children with epilepsy. Epilepsia. 1990;31(Suppl 4):S35-40.

30. Kaufman AS, Kaufman NL. Kaufman Assessment Battery for Children (K-ABC). Publishing, Circle Pines; 1983.

31. Scholte EM, van der Ploeg JD. Handleiding Sociaal-Emotionele Vragenlijst (SEV). Houten: Bohn Stafleu van Loghum; 2007.

32. Berkel A, et al: Handleiding voor het gebruik van de SDQ binnen de jeugdgezondheidszorg. Vragenlijst voor het signaleren van psychosociale problemen bij kinderen van 7-12 jaar. Zutphen: Markant congressen 2006.

33. MC Nair DM, Lorr M, Droppleman Lf. Profile of Mood States: Manual San Diego, CA: Educational and Industrial Testing Service; 1971.

34. Walker DK, Stein RE, Perrin EC, Jessop DJ. Assessing psychosocial adjustment of children with chronic illnesses: a review of the technical properties of PARS III. J Dev Behav Pediatr. 1990;11:116-21.

35. Hendriksen JG, Poysky JT, Schrans DG, Schouten EG, Aldenkamp AP, Vles JS. Psychosocial adjustment in males with Duchenne muscular dystrophy: psychometric properties and clinical utility of a parentreport questionnaire. J Pediatr Psychol. 2009;34:69-78.

36. Carpay HA, Vermeulen J, Stroink H, Brouwer OF, Peters AC, van Donselaar CA, Aldenkamp AP, Arts WF. Disability due to restrictions in childhood epilepsy. Dev Med Child Neurol. 1997;39:521-6.

37. Devilly GJ, Borkovec TD. Psychometric properties of the credibility/ expectancy questionnaire. J Behav Ther Exp Psychiatry. 2000;31:73-86.

38. EuroQol-a new facility for the measurement of health-related quality of life. The EuroQol Group. Health Policy. 1990;16:199-208.

39. Fekkes M, Theunissen NC, Brugman E, Veen S, Verrips EG, Koopman HM, Vogels T, Wit JM, VerlooveVanhorick SP. Development and psychometric evaluation of the TAPQOL: a health-related quality of life instrument for 1-5-year-old children. Qual Life Res. 2000;9:961-72.

40. Vogels T, Verrips GH, Koopman HM. TACQOL manual. Parent Form and Child Form Leiden: Leiden Center for Child Health and Pediatrics LUMC-TNO; 2000.

41. Goossens ME, Rutten-van Mölken MP, Vlaeyen JW, van der Linden SM. The cost diary: a method to measure direct and indirect costs in cost-effectiveness research. J Clin Epidemiol. 2000;53:688-95.

42. Oostenbrink JB, et al. Handleiding voor kostenonderzoek, methoden en standaard kostprijzen voor economische evaluaties in de gezondheidszorg. College voor zorgverzekeringen 2004.

43. Koopmanschap MA. PRODISQ: a modular questionnaire on productivity and disease for economic evaluation studies. Expert Rev Pharmacoecon Outcomes Res 2005, 5(1):23-8.

44. Dolan P, Gudex C, Kind P, Williams A. The time trade-off method: results from a general population study. Health Econ. 1996;5:141-54.

45. Lamers LM, Stalmeier PF, McDonnell J, Krabbe PF, van Busschbach JJ. Measuring the quality of life in economic evaluations: the Dutch EQ-5D tariff. Ned Tijdschr Geneeskd. 2005;149:1574-8.

46. Manca A, Hawkins N, Sculpher MJ. Estimating mean QALYs in trial-based cost-effectiveness analysis: the importance of controlling for baseline utility. Health Econ. 2005;14:487-96.

47. Barber JA, Thompson SG. Analysis of cost data in randomized trials: an application of the nonparametric bootstrap. Stat Med. 2000;19:3219-36. 
48. Ramsey S, Willke R, Briggs A, Brown R, Buxton M, Chawla A, Cook J, Glick H, Liljas B, Petitti D, Reed S. Good research practices for cost-effectiveness analysis alongside clinical trials: the ISPOR RCT-CEA Task Force report. Value Health. 2005;8:521-33.

49. Briggs AH, Wonderling DE, Mooney CZ. Pulling cost-effectiveness analysis up by its bootstraps: a nonparametric approach to confidence interval estimation. Health Econ. 1997;6:327-40. 


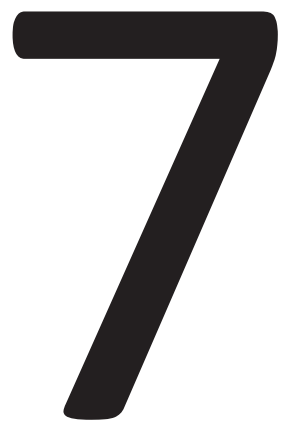

An economic evaluation of the ketogenic diet versus care as usual in children and adolescents with intractable epilepsy: an interim analysis

RJA de Kinderen, DAJE Lambrechts, BFM Wijnen, D Postulart, AP Aldenkamp, HJM Majoie, SMAA Evers

Submitted to Epilepsia 


\section{Abstract}

\section{Objectives}

To gain insight in the cost-effectiveness of the ketogenic (KD) diet compared with care as usual (CAU) in children and adolescents with intractable epilepsy, we conducted an economic evaluation from a societal perspective, alongside a randomized controlled trial.

\section{Methods}

Participants from a tertiary epilepsy center were randomized into KD (intervention) group or CAU (control) group. Seizure frequency, quality of life (QALYs), health care costs, production losses of parents and patient \& family costs were assessed at baseline and during a 4-month study period and compared between the intervention and control group. The incremental costeffectiveness (i.e. cost per responder), cost-utility (i.e. cost per QALY) and cost-effectiveness acceptability curves CEACs were calculated and presented.

\section{Results}

In total, 48 children were included in the analyses of this study (26 KD group). At 4 months, $50 \%$ of the participants in the KD group had a seizure reduction $>50 \%$ from baseline, compared with $18.5 \%$ of the participants in the CAU group. There is a $93 \%$ probability of the ketogenic diet being cost-effective when looking at the cost per responder, at a ceiling ratio of $€ 50,000$ based on our cost-effectiveness acceptability curve. We failed, however, to measure any benefits in terms of QALYs and therefore, the cost per QALY rise high above any acceptable ceiling ratio. Univariate and multivariate sensitivity analyses and non-parametric bootstrapping was performed, and demonstrated the robustness of our results.

\section{Significance}

The results show that the KD reduces seizure frequency and is a cost-effective treatment option looking at the cost per responder $(€ 18,044)$. The study did not find any improvements in quality of life and, therefore, unfavorable cost-utility ratios resulted. 


\section{Introduction}

Epilepsy is a major, cost-intensive and global health problem. ${ }^{1}$ In the Netherlands, the prevalence of epilepsy is estimated to be five per 1,000 of the population; at any one time, about 80,000 individuals have epilepsy. ${ }^{2}$ In 2004 , the total costs of epilepsy in Europe were $€ 15.5$ billion, indirect costs being the single most dominant cost category (€8.6 billion). ${ }^{3}$ In 2011, in the Netherlands, the direct medical costs were $€ 248$ million, which is $0.3 \%$ of the health care budget. ${ }^{4}$ Although in the majority of the patients epilepsy is treatable with antiepileptic drugs (AEDs), about $30 \%$ of the cases suffer from intractable epilepsy. ${ }^{5}$ Patients are diagnosed as having intractable epilepsy when their seizures are not controlled after taking two or more different AEDs. Patients with uncontrolled epilepsy depend heavily on informal care (family and friends), and on health care professionals (neurologists, social workers, psychologists etc.). Complications due to intractable epilepsy result in frequent hospitalizations and many of these patients are institutionalized. Living with uncontrolled seizures has a negative impact on the Quality of Life (QoL) of the child and his/her surrounding.

Patients with intractable epilepsy after failure of antiepileptic drugs and/or resective surgery can potentially benefit from a ketogenic diet (KD). The KD is a high-fat, lowcarbohydrate diet that imitates the metabolic state of fasting while maintaining a normal number of calories. Body energy requirements while on a KD are met by lipolysis and ß-oxidation of fatty acids rather than by the breakdown of carbohydrates. The exact anticonvulsant mechanism of action of a KD has not yet been elucidated. ${ }^{6}$ Currently, the scientific and clinical attention paid to the role of the KD is negligible. ${ }^{7}$ This means that a KD is often overlooked and underutilized as a treatment option for children with intractable epilepsy. An important reason for this is that relatively few children and their parents can comply with the stringent diet. Physicians are, therefore, often reluctant to initiate the diet. The beneficial effect of a $K D$ has been studied in multiple observational studies ${ }^{8-16}$, reviews ${ }^{17-21}$ and in two randomized controlled trials. ${ }^{22,23}$ However, none of these studies has researched the cost-effectiveness of the KD. Since resources are scarce, in current health care decision-making, we need to prove the effects of the KD are worth the extra costs, ${ }^{24}$ to allow decisions to be evidence-based. ${ }^{25}$ Economic evaluation is a method for examining this trade-off between costs and effects of comparative treatments. In order to inform the decision makers, full (or good) economic evaluations are necessary. Therefore, this trial-based economic evaluation researches the cost-utility and cost-effectiveness of the KD compared with care as usual (CAU) in children and adolescents with intractable epilepsy from a societal perspective, alongside a randomized controlled clinical trial. 


\section{Methods}

The economic evaluation was executed alongside a randomized controlled clinical trial; details of the trial design can be found elsewhere. ${ }^{26}$ In short, between July 2010 and September 2014, subjects were randomized to either the KD or to CAU after a 1-month baseline period. Patients randomized into the KD group were followed during the 4-month comparative study period and a follow-up period of 12 months. Patients randomized into the CAU group were followed only during the 4-month study period. The timeline of the study is graphically presented in Figure 7.1. This interim analysis reports the cost-effectiveness of the comparative period of the trial, i.e. the 4-month study period where direct comparison between KD and CAU is possible. This study has been approved by the ethics committee of the Academic Medical Center Utrecht, the Netherlands.

The economic evaluation was performed from a societal perspective and consisted of a cost-utility analysis with QALY as primary outcome measure and a cost-effectiveness analysis where treatment responder (i.e. $\geq 50 \%$ seizure reduction) is the primary outcome measure.

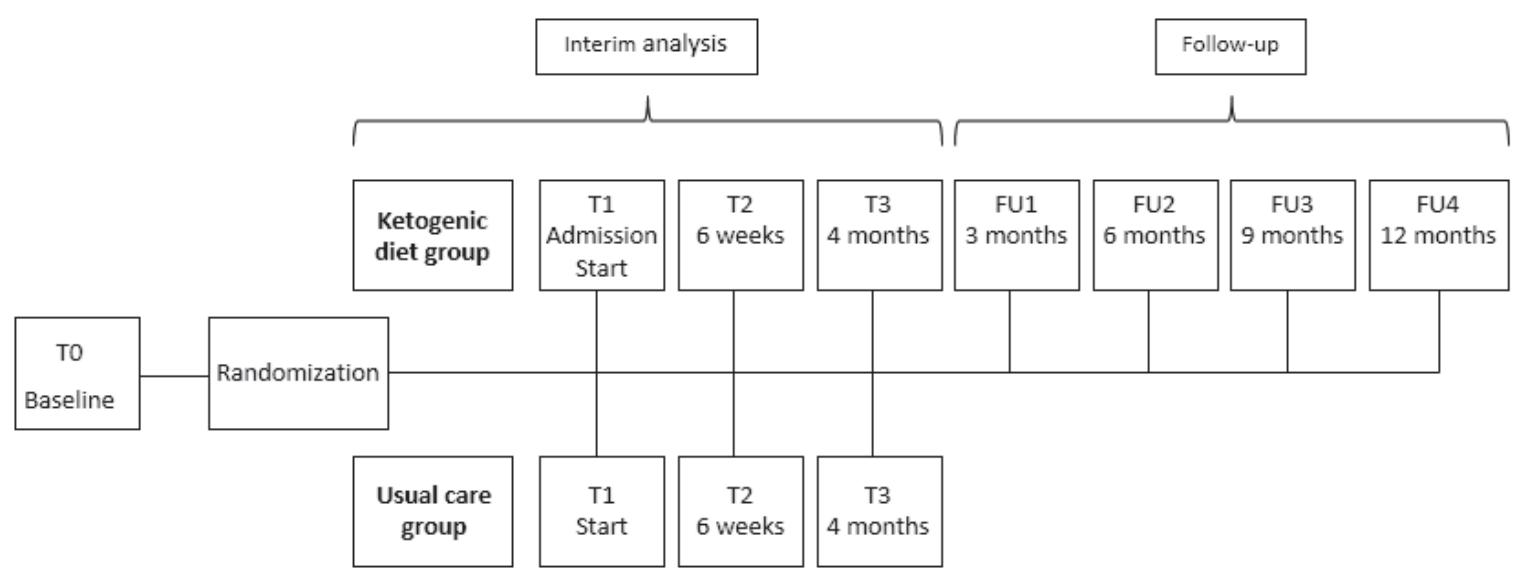

Figure 7.1 Flowchart study design.

\section{Study population and sample size}

The patient population consisted of children and adolescents with intractable epilepsy. Patients were eligible to participate if they met the following criteria: age between 1 and 18 years; having uncontrolled seizures after trying two or more appropriate AEDs (i.e. intractable epilepsy); not eligible for epilepsy surgery; no fatty acid oxidation disorders and related diseases; no diabetes and hyperinsulinism; no prolonged QT-time syndrome; no hypercholesterolemia or hypertriglyceridemia; no severe liver, kidney or pancreas diseases; no renal tubular acidosis; no severe behavioral disorder; no malnutrition; no treatment with Topiramate or Acetazolamide and no positive family history or other risk factors for kidney stones or acidosis. Eligible candidates and their parents were referred by their attending physician to the 
multidisciplinary KD team at the epilepsy center Kempenhaeghe in the Netherlands, where they received detailed information about the study before giving their consent. Based on a minimum detectable difference in success rate of $35 \%$ between the KD group and usual care, and assuming that alpha $=5 \%$ and power $=80 \%$, we needed 22 children for each group. ${ }^{26}$

\section{Interventions}

\section{Ketogenic diet}

Patients assigned to the KD group were admitted to the tertiary epilepsy center for five days, while KD was introduced by a dietician. The neurologist, pediatrician and epilepsy nurse visited the child during the 5-day admission and ketosis was checked daily in urine (if toilet-trained) and three times in blood by means of a finger puncture. The AEDs used by the children and adolescents at the time of inclusion in the study were continued without changes during the study period (except when medically indicated). The dietician decided together with the parents whether the classical diet, the medium-chain triglyceride (MCT) or a mixture of both diets was introduced. Children who were tube-fed were also treated with KD. The diet was then adjusted to a fluid version.

After the 5-day admission, weekly telephone meetings between parents and the epilepsy nurse, and between parents and the dietician, were planned. Ketosis was checked daily by the parents in urine (if possible) or three times a week in blood via a finger puncture. Furthermore, time points T2 and T3 consisted of a visit to the neurologist, pediatrician, dietician and epilepsy nurse. At T3, blood and urine samples were taken from the patients in the intervention group. No other protocol-driven care was delivered.

\section{Care as usual}

Patients randomized to CAU continued to take their AEDs. The controls were treated and monitored according to good clinical practice. Patients randomized into the control group were seen at time points T2 and T3 by a neurologist, pediatrician and epilepsy nurse. Weekly telephone meetings were scheduled between parents and the epilepsy nurse. No other protocol-driven care was delivered.

\section{Outcome measures}

\section{Seizure frequency}

A daily seizure calendar was used to record seizures continuously. Types of seizures were described and labelled in accordance with the International League Against Epilepsy (ILAE) classification. A patient was labeled as a responder when he/she had a 
mean reduction in seizures of $50 \%$ or more during the last month of the study period (month 4) compared with baseline.

\section{Utilities}

Generic QoL of both the children and their parents was measured. For this purpose the EuroQol instrument (EQ-5D) ${ }^{27}$ and the EQ-5D-Y version for children aged eight years and older was used. ${ }^{28}$ The EQ-5D health states were converted to a single summary index between 0 and 1 (utility) by applying the Dutch utility-tariff. ${ }^{29}$ In addition to this, QoL was also assessed by the TAPQOL (TNO-AZL Preschool Children's Quality of Life) for children aged between 1 and 5 years (parent proxy) ${ }^{30}$ and the TACQOL (TNO-AZL Children's Quality of Life) for children aged between 6 and 16 years (parent proxy). ${ }^{31}$ The TAPQOL has nine domains, all ranging between 0 and 100 . The TACQOL has seven domains, two of which have scores ranging between 0 and 32 and two domains between 0 and 16 . All domains were given equal weight factors; a mean score was calculated and transformed into a score between 0 and 1 in order to represent a utility. Utility scores from the EQ-5D, EQ-5D-Y, TAPQOL and TACQOL were measured at time points TO and T3.

QALYs were calculated by means of the 'under the curve method', in which the time in a certain health state was multiplied by the utility of this health state. ${ }^{24}$

\section{Costs}

Costs were calculated from a societal perspective; therefore, protocol-driven intervention costs, other health care costs, patient and family costs, and productivity losses of the parent(s) were measured. Costs were expressed in Euros and were converted (if necessary) to the price year 2013. Due to the time horizon of 4 months, no discounting was performed.

Other (epilepsy-related) health care costs and patient and family costs were measured by a monthly cost diary, ${ }^{32}$ completed by the patient's parent(s). Additional costs consisted of parents' productivity losses, based on the hours absent from work due to their child's epilepsy or their own health-related problems.

Costs were estimated using a bottom-up approach, in which information on each element of service used was multiplied by an appropriate standardized unit cost and summed to provide an overall total cost. ${ }^{24}$ Where possible, unit costs were based on standardized costs from the Dutch guidelines for cost research. ${ }^{33}$ Real tariffs or costs were used when no standardized unit costs were available. Cost of medication was calculated using guideline prices. ${ }^{33}$ An addition for prescription charges for prescribed medication was taken into account. When data on medication were diverse, lowest cost prices for the specific medication were used. The cost of informal care was calculated using shadow pricing. For this, the general hourly minimum wages were applied. ${ }^{33}$ Productivity costs were calculated on the basis of the Friction Cost Method (FCM), following the Dutch guidelines. ${ }^{33}$ The FCM is based on the assumption that an organization needs a certain time span (friction period) to replace the absent worker 
by another worker. The definitive number of days absent from work is then limited to the duration of the friction period, which was determined in the Netherlands to be 23 weeks in $2010 .^{33}$ Unit prices are presented in Table 7.1.

Table $7.1 \quad$ Unit prices.

\begin{tabular}{|c|c|c|}
\hline & & $€(2013)$ \\
\hline \multirow[t]{2}{*}{ Intervention costs (4 months) } & KD group & $4311.81^{b}$ \\
\hline & CAU group & $1548.60^{b}$ \\
\hline \multirow[t]{4}{*}{ Diet costs (4 months) } & Classical diet & 0 \\
\hline & MCT diet & $2491.20^{b}$ \\
\hline & Tube diet & $3476.40^{b}$ \\
\hline & Mixture & $1246.60^{b}$ \\
\hline \multirow[t]{3}{*}{ Ketosis check } & Finger puncture device & $21.73^{b}$ \\
\hline & In blood (finger puncture) & $2.02^{b}$ \\
\hline & In urine & $0.14^{b}$ \\
\hline \multirow[t]{21}{*}{ Health care costs } & GP visit & $30.48^{a}$ \\
\hline & Specialist visit & $140.42^{a}$ \\
\hline & Epilepsy nurse visit & $109.65^{b}$ \\
\hline & Blood sample & $24.77^{b}$ \\
\hline & Physiotherapist visit & $39.19^{a}$ \\
\hline & Speech therapist visit & $35.92^{\mathrm{a}}$ \\
\hline & Dietician visit & $29.39^{\mathrm{a}}$ \\
\hline & Other paramedic visit & $23.95^{\mathrm{a}}$ \\
\hline & Psychologist visits & $87.08^{a}$ \\
\hline & Psychiatrist visit & $112.12^{\mathrm{a}}$ \\
\hline & Routine EEG & $273.55^{b}$ \\
\hline & 24-hours EEG & $779.65^{b}$ \\
\hline & MRI & $179.84^{b}$ \\
\hline & General hospital (day) & $473.50^{\mathrm{a}}$ \\
\hline & Academic hospital (day) & $625.89^{a}$ \\
\hline & Epilepsy center (day) & $208.25^{b}$ \\
\hline & Inpatient disabled care (day) & $208.25^{b}$ \\
\hline & Day care & $273.21^{\mathrm{a}}$ \\
\hline & Home care (hour) & $70.75^{a}$ \\
\hline & Social services (hour) & $70.75^{\mathrm{a}}$ \\
\hline & Prescribed medication & Variable $^{c}$ \\
\hline \multirow[t]{6}{*}{ Patient and family costs } & Informal care (hour) & $13.61^{\mathrm{a}}$ \\
\hline & Child care & Variable $^{b}$ \\
\hline & Housekeeping (hour) & $13.61^{a}$ \\
\hline & Medication (OTC) & Variable $^{c}$ \\
\hline & Activities & Variable $^{b}$ \\
\hline & Transport (per kilometer) & $0.20^{\mathrm{a}}$ \\
\hline Production losses & Per hour & $32.68^{\mathrm{a}}$ \\
\hline
\end{tabular}

${ }^{a}$ Dutch guidelines for costing studies; ${ }^{b}$ (Calculated from) respective providers or professional organizations;

${ }^{\mathrm{c}}$ Dutch Pharmaceutical Therapeutic Compass.

\section{Data analyses}

The economic analyses were carried out on an intention to treat (ITT) protocol. Mean imputation was used to impute missing Quality Adjusted Life Years (QALYs) and cost data within the treatment group. 
Data were analyzed using IBM SPSS Statistics version 22. Baseline differences in costs and utilities between groups were checked with nonparametric bootstrapping, based on 1,000 bootstrap replications, as the data did not comply with the underlying assumptions of parametric tests.

Furthermore, the arithmetic mean and 95\% nonparametric confidence intervals were calculated (1,000 bootstrap replications) for differential costs and QALYs during the study period.

Additionally, bootstrapping with Microsoft Excel 2010 was used to quantify the uncertainty around the Incremental Cost Effectiveness Ratio (ICER) (5,000 bootstrap replications). The ICERs were calculated by dividing the incremental costs by the incremental QALYS. The ICER represents the cost of an additional QALY gained and was used to estimate the cost-utility of KD as opposed to CAU. The ICERs were presented on a cost-effectiveness (CE-)plane, which is divided into four quadrants. The south-east quadrant represents the instances where the intervention is more effective and less costly than the comparators (dominates), with the opposite situation in the north-west quadrant, representing interventions that are more costly and less effective than comparators (dominated). The north-east quadrant represents interventions that are more costly yet more effective, while the south-west quadrant represents less effective and less costly interventions. ${ }^{24} \mathrm{~A}$ treatment is deemed costeffective when it is dominant or when the costs per QALY are below a specified ceiling ratio. In the Netherlands, an informal ceiling ratio exists between approximately $€ 20,000$ and $€ 80,000$ per QALY, depending on the burden of disease. ${ }^{34}$ The disability weight of severe epilepsy is estimated to be $0.6577^{35}$ therefore, a threshold of $€ 50,000$ was taken as a ceiling ratio in this study. ${ }^{34}$ To show the probability that a KD is costeffective, given different ceiling ratios, a Cost-Effectiveness Acceptability Curve (CEAC) was constructed. ${ }^{24}$ Furthermore, the number of responding patients (i.e. $\geq 50 \%$ seizure reduction) and the incremental cost per responding patient were calculated.

A univariate and a multivariate sensitivity analysis was performed to explore how robust the results were to changes in the assumptions upon which they were based. Firstly, we conducted the economic evaluation from a health care perspective in order to comply with the guidelines of other policy bodies. ${ }^{36}$ Secondly, we explored the impact on the cost-effectiveness of the KD by varying the cost of the intervention and diet, as these costs are mainly based on the specific protocol of the epilepsy center Kempenhaeghe. Other centers initiate the KD during outpatient visits and offer only the classical diet, possibly due to reimbursement issues.

\section{Results}

Between August 2010 and September 2014, a total of 58 patients were included in the study. One patient dropped out of the study before randomization could take place and nine patients dropped out of the study: six from the control group, due to 
dissatisfaction regarding the randomization result, and three patients from the KD group, due to spontaneous seizure reduction (one patient) and protocol violation (two patients), leaving 48 patients in the ITT analysis.

There were no statistical differences between the KD and CAU group at baseline in utilities (both TACQOL/TAPQOL and EQ-5D) or in total health care, patient \& family and productivity losses. Therefore, baseline correction was assumed to be unnecessary. Demographic data and baseline utilities are presented in Table 7.2.

\section{Outcomes}

In total, 13 patients from the KD group (50\%) and 4 patients from the CAU group (18.2\%) were responders ( $\geq 50 \%$ seizure reduction). Measuring utilities with the EQ-5D-Y seemed only possible in a minority of our patient sample. We, therefore, used the utility-scores of the TAPQOL and TACQOL (age-dependent) to calculate QALYs for the patients. The QALYs of the parents were based on utilities of the EQ-5D. No significant differences were found in patients' QALYs nor in parents' QALYS between the two groups (Table 7.3). Total health care costs differed significantly between the two groups due to significantly higher intervention and diet costs in the KD group. No other significant cost differences were found.

\section{ICERs}

The CE-plane for the cost-utility analysis, representing the uncertainty surrounding the cost per QALY ratio, is shown in Figure 7.2A. As the KD is more expensive than CAU (resp. $€ 20,986$ and $€ 15,245$ ) and (almost) equally effective in terms of QALYs (resp. 0.253 and 0.250), most bootstrapped ICERs lie in the northern part of the CE-plane ( $40 \%$ north-west and $56 \%$ north-east). Based on the cost-utility analysis, the $K D$ is not a cost-effective treatment option. Figure 7.2B presents the CEAC based on the cost per QALY, showing the probability that the KD is cost-effective at different ceiling ratios. At the ceiling ratio of $€ 50,000$, there is only a $5 \%$ probability of the KD being cost-effective. 
Table 7.2 Demographic characteristics and baseline utilities.

\begin{tabular}{|c|c|c|}
\hline & $\begin{array}{c}\mathrm{KD} \mathrm{N}=26 \\
\mathrm{~N}(\%)\end{array}$ & $\begin{array}{c}\text { CAU N=22 } \\
N(\%)\end{array}$ \\
\hline Female & $8(30.8)$ & $13(59.1)$ \\
\hline Age mean (range) & $7.8(2.1-16.5)$ & $8.1(1.1-15.7)$ \\
\hline Age seizure onset (range) & $2.4(0-8)$ & $1.9(0-10)$ \\
\hline \multicolumn{3}{|l|}{ Etiology } \\
\hline Genetic & $9(34.6)$ & $1(4.5)$ \\
\hline Structural & $2(7.7)$ & $10(45.5)$ \\
\hline Unknown & $15(57.7)$ & $11(50.0)$ \\
\hline \multicolumn{3}{|l|}{ Full scale IQ } \\
\hline$<50$ & $10(38.5)$ & $11(50.0)$ \\
\hline $50-69$ & $3(11.5)$ & $4(18.2)$ \\
\hline $70-99$ & $11(42.3)$ & $5(22.7)$ \\
\hline$\geq 100$ & $2(7.7)$ & $2(9.1)$ \\
\hline \multicolumn{3}{|l|}{ Behavioral/Cognitive problems } \\
\hline ADHD & $0(0.0)$ & $1(4.5)$ \\
\hline ADD & $1(3.8)$ & $1(4.5)$ \\
\hline Autism & $3(11.5)$ & $5(22.7)$ \\
\hline VNS at start study & $1(3.8)$ & $0(0.0)$ \\
\hline \multicolumn{3}{|l|}{ Number of AEDs at start study } \\
\hline None & $0(0.0)$ & $1(4.5)$ \\
\hline One & $3(11.5)$ & $4(18.2)$ \\
\hline Two & $12(46.2)$ & $8(36.4)$ \\
\hline Three & $9(34.6)$ & $5(22.7)$ \\
\hline Four & $2(7.7)$ & $2(9.1)$ \\
\hline Five & $0(0.0)$ & $2(9.1)$ \\
\hline \multicolumn{3}{|l|}{ Diet type } \\
\hline $\mathrm{MCT}$ & $18(69.2)$ & -- \\
\hline Classical & 7 (26.9) & -- \\
\hline Mixture & $1(3.8)$ & - \\
\hline PGT & $6(23.1)$ & $5(22.7)$ \\
\hline \multicolumn{3}{|l|}{ Mean quality of life } \\
\hline TACQOL or TAPQOL & 0.77 & 0.75 \\
\hline EQ-5D-youth ${ }^{+}$ & 0.53 & 0.64 \\
\hline \multicolumn{3}{|l|}{ Number AEDs tried in the past } \\
\hline Three & $3(11.5)$ & $4(18.2)$ \\
\hline Four & $4(15.4)$ & $5(22.7)$ \\
\hline Five & $9(34.6)$ & $3(13.6)$ \\
\hline Six & $3(11.5)$ & $3(13.6)$ \\
\hline Seven & $2(7.7)$ & $1(4.5)$ \\
\hline Eight & $4(15.4)$ & $2(9.1)$ \\
\hline Nine & $1(3.8)$ & $4(18.2)$ \\
\hline VNS in the past & $1(3.8)$ & $1(4.5)$ \\
\hline Epilepsy surgery in the past & $1(3.8)$ & $0(0.0)$ \\
\hline
\end{tabular}

† Based on N=10 KD group and N=11 CAU group, KD: Ketogenic diet, CAU: Care as usual, N: Number, AEDs: Antiepileptic drugs, ADHD: Attention deficit hyperactivity disorder, ADD: Attention deficit disorder, VNS: Vagus nerve stimulator, MCT: Multi chain tryglyceride, PGT: percutaneous gastrostomy tube. 


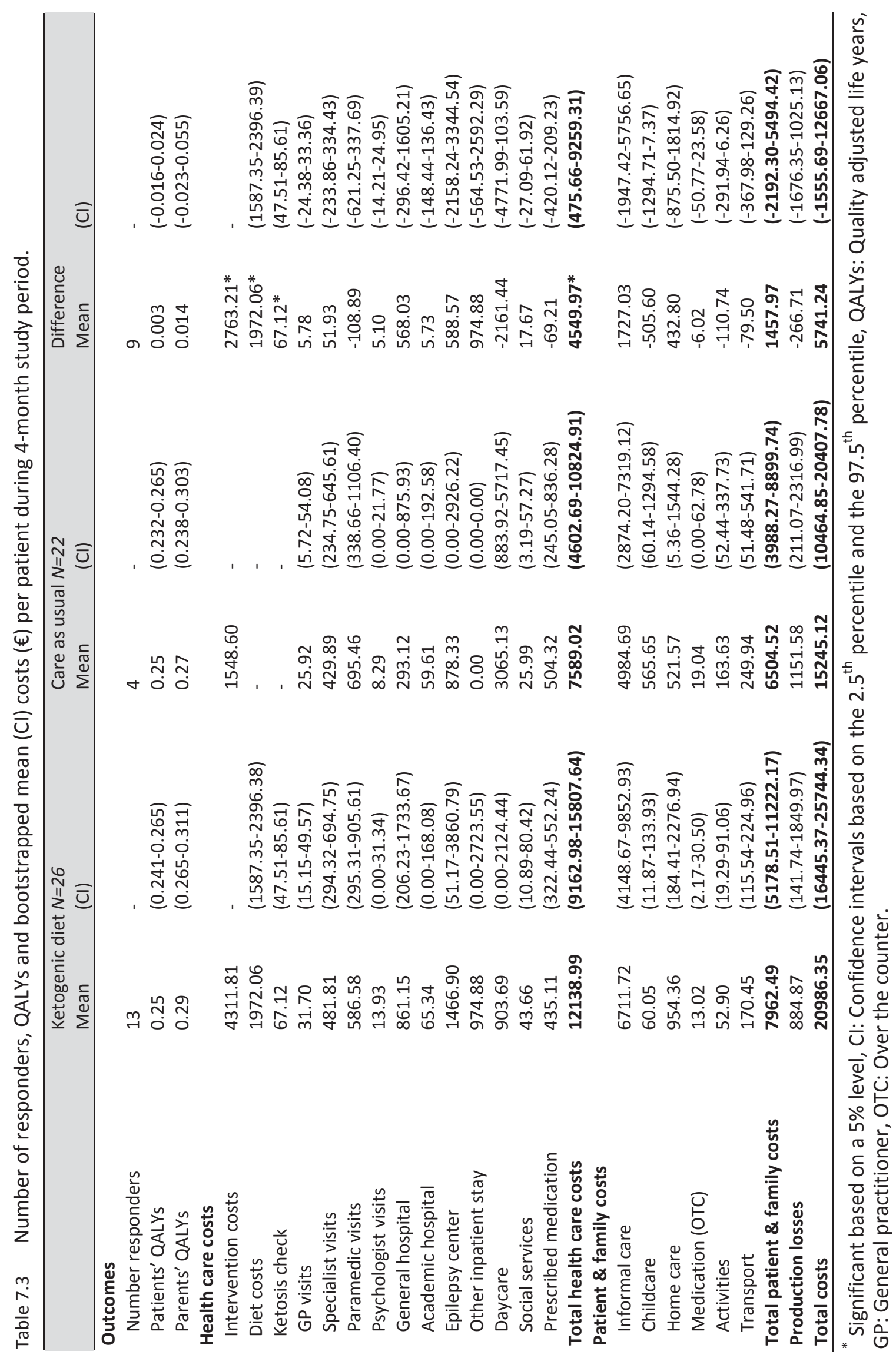


A

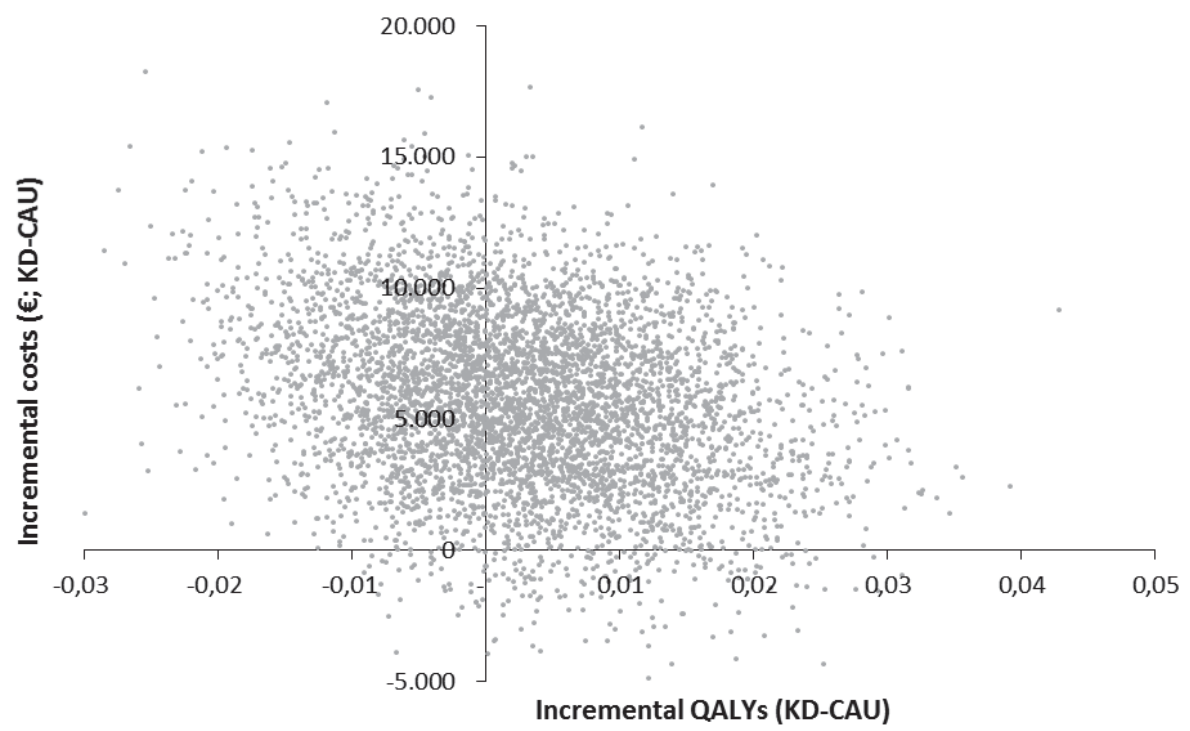

B

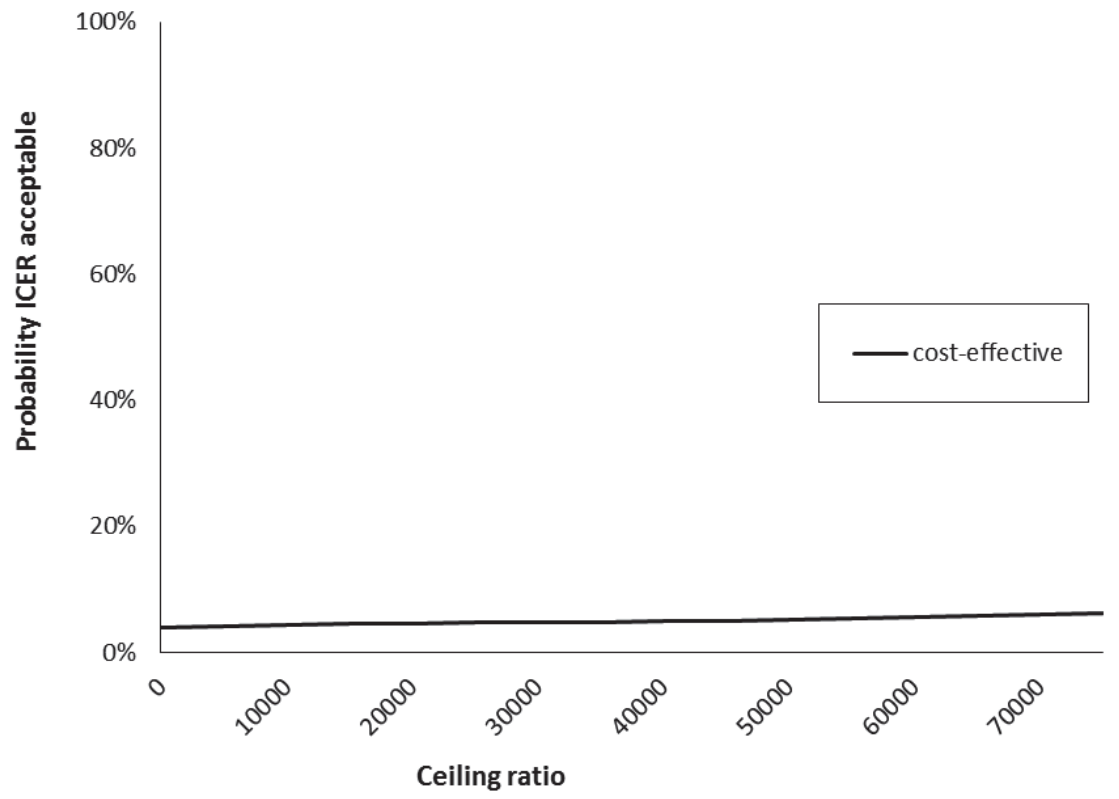

Figure 7.2 (A) Cost-effectiveness plane, cost per QALY, (B) Cost-effectiveness acceptability curve, cost per QALY.

The ICER of the cost per responder is $€ 18,044$. The uncertainty analysis of this ICER is presented in the CE-plane in Figure 7.3A. Now, most ICERs lie in the north-east quadrant, indicating that the KD is more expensive but also more effective than CAU in children and adolescents with intractable epilepsy. At a ceiling ratio of $€ 50,000$ per responder, there is a $93 \%$ probability that the $\mathrm{KD}$ is cost-effective. Figure $7.3 \mathrm{~B}$ presents the CEAC based on the number of responders, demonstrating the probability that the KD is cost-effective at different ceiling ratios. 
A

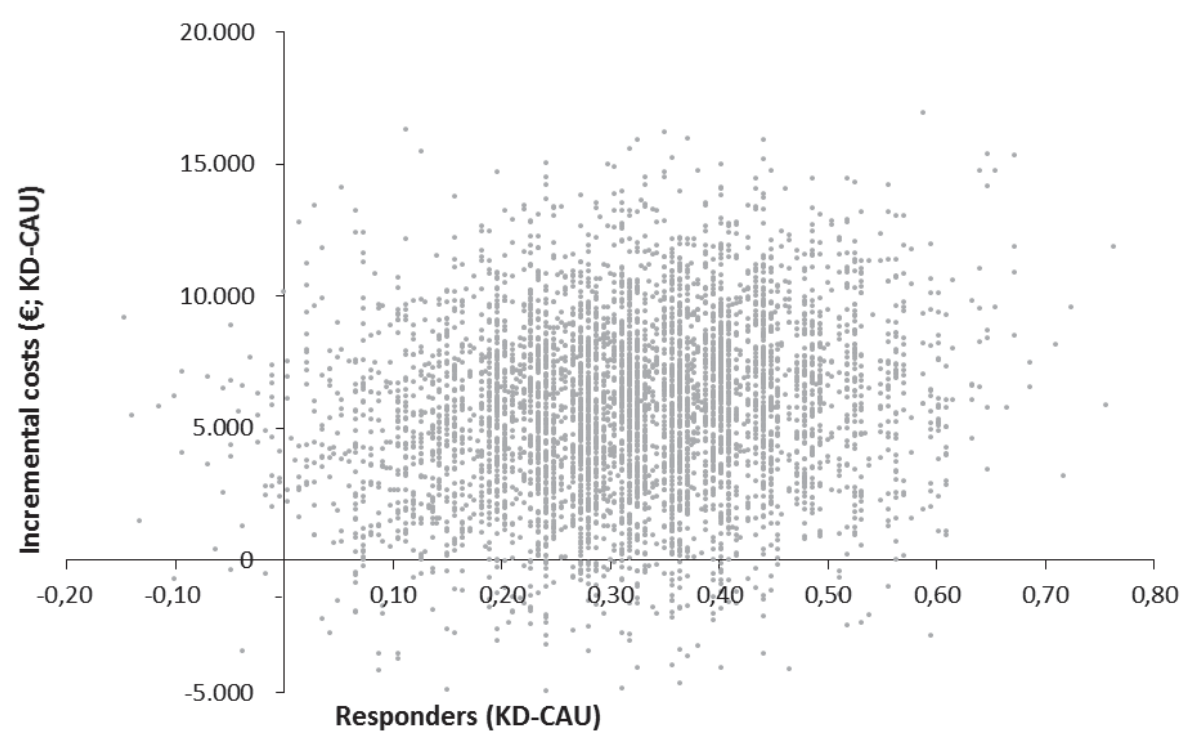

B

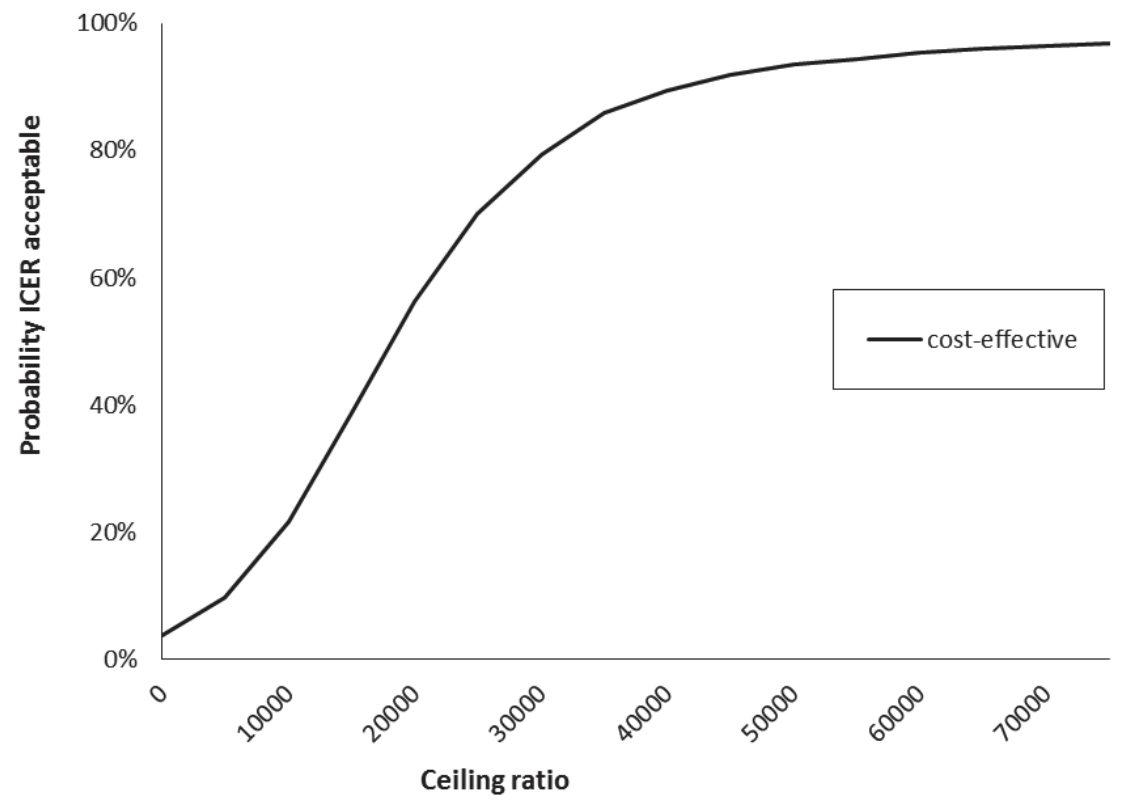

Figure 7.3 (A) Cost-effectiveness plane, cost per responder, (B) Cost-effectiveness acceptability curve, cost per responder.

\section{Sensitivity analyses}

Firstly, performing the analyses from a health care perspective and at a ceiling ratio of $€ 50,000$, results showed that KD has a $96 \%$ probability of being cost-effective when looking at the cost per responder, and only 3\% probability when looking at the cost per QALY (CE-planes and CEACs not presented).

Secondly, lowering the cost of the intervention by initiating the KD without the 5-day admission to the epilepsy center, ignoring the telephone meetings with dietician and/or nurse (in both KD and CAU groups) and simultaneously lowering the cost of 
the KD by offering solely the classical variant of the diet, results in the KD being costeffective at any ceiling ratio, when looking at the cost per responder from a societal perspective (ICER: $€ 5,544$ ) (Figure 7.4A). However, looking at the cost per QALY, the KD still only has a $32 \%$ probability of being cost-effective at a ceiling ratio of $€ 50,000$ per QALY (Figure 7.4B).

A

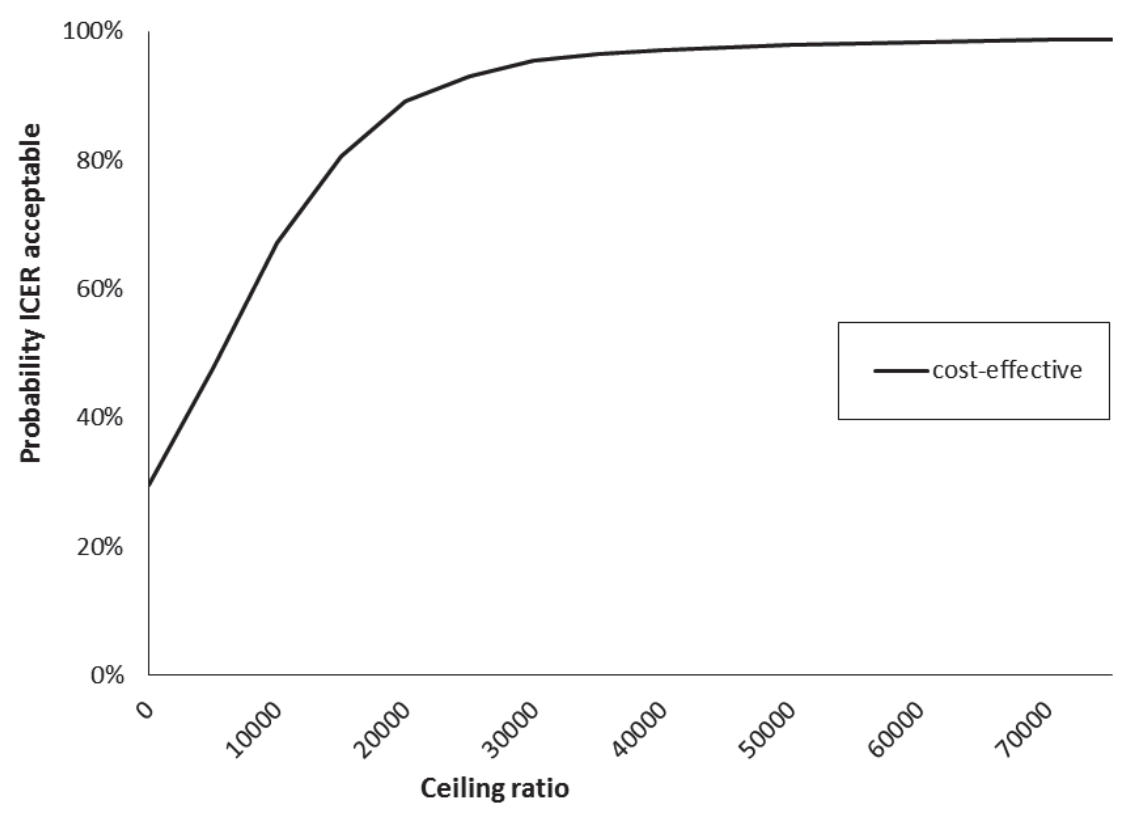

B

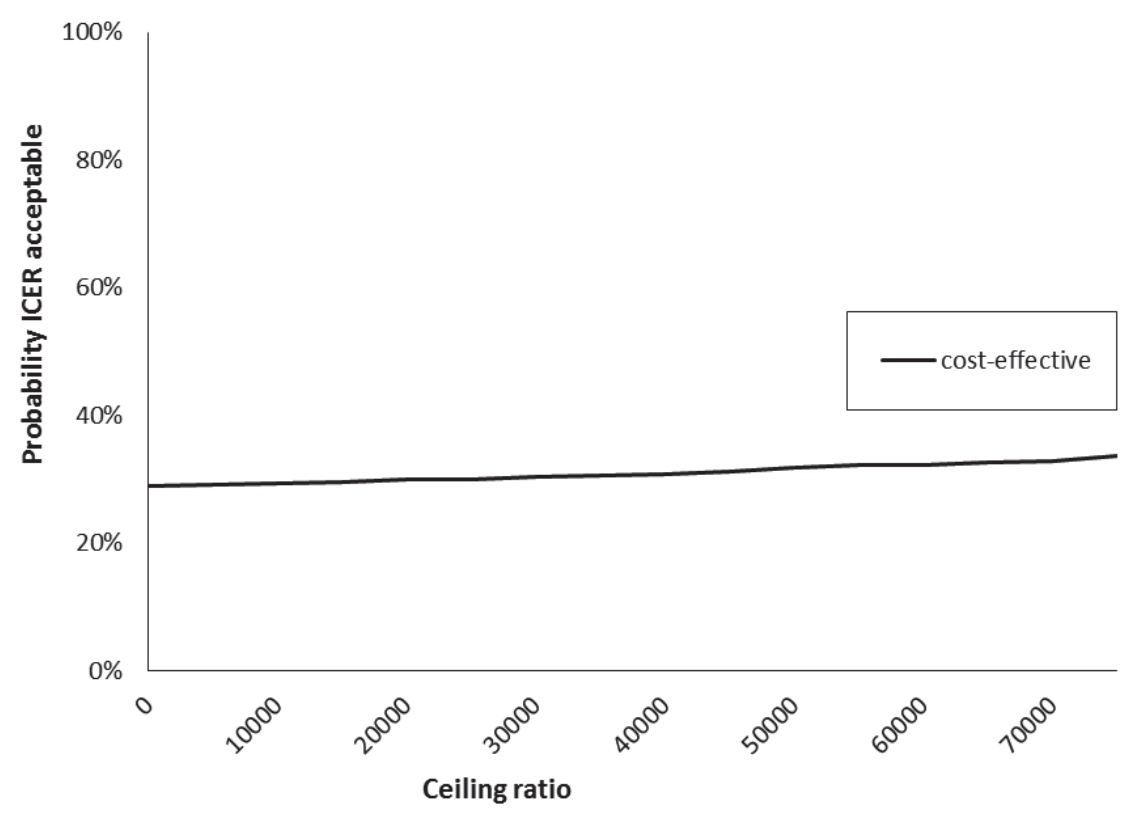

Figure 7.4 (A) Cost-effectiveness acceptability curve, cost per responder, sensitivity analysis, (B) Costeffectiveness acceptability curve, cost per QALY, sensitivity analysis. 


\section{Discussion}

To our knowledge, this is the first study reporting an economic evaluation comparing KD with CAU in children and adolescents with intractable epilepsy alongside a randomized controlled trial. The economic evaluation was conducted from a societal perspective.

The KD group is, as far as number of responders is concerned, more effective than CAU: resp. $50 \%$ and $18.2 \%$, but fails to show an increase in QALYs.

Looking at the cost per responder, there is a 93\% probability that the KD is costeffective at a ceiling ratio of $€ 50,000$. However, this 4-month analysis shows that the KD is not cost-effective when including the QALY as a primary outcome parameter, as the corresponding ICERs of the cost-utility analysis rise high above any acceptable ceiling ratio in the Netherlands.

Our results are, however, sensitive to change, as can been seen in the sensitivity analyses, where we hypothetically decreased the intervention costs, and simultaneously increased the classical diet users from $27 \%$ to $100 \%$. This resulted in a $98 \%$ probability for KD to be cost-effective at a ceiling ratio of $€ 50,000$ when looking at the cost per responder and in a 32\% probability when looking at the cost per QALY. This study has some limitations. We were aiming at calculating the cost per QALY, as this is essential information for policy makers. However, there is no preference-based generic QoL instrument available for utility measurements in children. In order to be able to calculate QALYs, a utility-proxy between 0 and 1 was used, which we adapted from the scores of the age-dependent TAPQOL and TACQOL. The 'utility'-scores we used to calculate QALYs, are, therefore, not preference-based utilities and subsequently not comparable with other 'real' utilities. Secondly, as the TAPQOL and TACQOL are age-dependent measures, our used utility-proxies consist of a combination of both measures, limiting the comparability of utility measurement among different age groups.

On the one hand, the question arises whether the QoL instrument used in our study was sensitive enough to measure clinical changes (e.g. being a responder). Perhaps we would be successful in measuring an increase in QALYs relating to the number of responders if we had a preference-based generic quality of life instrument to be used in children of all ages. Half of the participants in our study were children and adolescents with combinations of severe intellectual and physical disabilities for which measuring quality of life is an overall challenge. ${ }^{37}$ On the other hand, being a responder may not be enough to gain QoL.

The third limitation, is the short time horizon of the study which can be the reason for our inability to measure change in QALYs or costs. This analysis compares the KD group with the CAU group over a period of 4 months. Although a longer comparative period would be preferable, this was not possible due to ethical considerations. The significant differences in costs that we found were fully related to the intervention and diet costs in the KD group. We did not find any reduction in health care costs, 
patient \& family costs or in productivity losses. Finally, transferability problems of our results to other centers within and outside the Netherlands may arise, as volumes and costs reported are (partly) related to the protocol of the tertiary epilepsy center Kempenhaeghe.

Until now, two other RCTs comparing KD with CAU can be found in the published literature. ${ }^{22,23}$ Comparing the results of this study with those from the RCT reported by Neal et al. ${ }^{22}$ and Sharma et al. ${ }^{23}$, showed that we found a higher percentage of responders (50\%) in the KD group compared to Neal et al. (38\%) and a slightly lower percentage of responders than Sharma et al. (52\%). Comparing the difference, our number of 'responders' (18.5\%) in the control group was larger than those reported by Neal et al. (6\%) or by Sharma et al. (11.5\%). Our cost-utility results are in line with a recently published model-based economic evaluation in which the KD is compared to vagus nerve stimulation (VNS) and CAU. ${ }^{38}$ Based on the results of this cost-utility Markov model, neither KD nor VNS are cost-effective options compared to CAU (€346,899 and €641,068 per QALY, respectively). Furthermore, Mackay et al. estimated the costs of three and 12 months KD to be AUS\$3,879 and AUS\$7,275, respectively. ${ }^{39}$

Despite the reported limitations, this study presents evidence of an economic evaluation executed alongside a RCT on the KD. We have shown that the KD has efficacy and efficiency when taking the number of responders into account. The study failed, however, to show any improvements in QoL and, therefore, unfavorable costutility ratios resulted. 


\section{References}

1. Strzelczyk A, Reese JP, Dodel R, Hamer HM. Cost of epilepsy: a systematic review. Pharmacoeconomics. 2008;26:463-76.

2. Wallace $H$, Shorvon S, Tallis R. Age-specific incidence and prevalence rates of treated epilepsy in an unselected population of 2,052,922 and age-specific fertility rates of women with epilepsy. Lancet. 1998;352:1970-3..

3. Pugliatti M, Beghi E, Forsgren L, Ekman M, Sobocki P. Estimating the cost of epilepsy in Europe: a review with economic modeling. Epilepsia. 2007;48:2224-33.

4. Slobbe LCJ, Smit JM, Groen J, Poos MJJC, Kommer GJ. Cost of Illness in the Netherlands 2007: Trends in healthcare expenditure 1999-2010. Bilthoven: RIVM; 2011.

5. Sillanpaa M, Schmidt D. Natural history of treated childhood-onset epilepsy: prospective, long-term population-based study. Brain. 2006;129:617-24.

6. Freeman J, Veggiotti P, Lanzi G, Tagliabue A, Perucca E. The ketogenic diet: from molecular mechanisms to clinical effects. Epilepsy Res. 2006;68:145-80.

7. MatthewsFriends, editor. 4th Global symposium for Dietary Thearpies for Epilepsy and other Neurological Disorders for Health Care Professionals; 2014; Liverpool

8. Caraballo RH, Cersosimo RO, Sakr D, Cresta A, Escobal N, Fejerman N. Ketogenic diet in patients with Dravet syndrome. Epilepsia. 2005;46:1539-44.

9. Coppola G, Veggiotti P, Cusmai R, Bertoli S, Cardinali S, Dionisi-Vici C, et al. The ketogenic diet in children, adolescents and young adults with refractory epilepsy: an Italian multicentric experience. Epilepsy Res. 2002;48:221-7.

10. Freeman JM, Vining EP, Pillas DJ, Pyzik PL, Casey JC, Kelly LM. The efficacy of the ketogenic diet-1998: a prospective evaluation of intervention in 150 children. Pediatrics. 1998;102:1358-63.

11. Kang HC, Kim YJ, Kim DW, Kim HD. Efficacy and safety of the ketogenic diet for intractable childhood epilepsy: Korean multicentric experience. Epilepsia. 2005;46:272-9.

12. Kankirawatana $P$, Jirapinyo $P$, Kankirawatana $S$, Wongarn $R$, Thamanasiri N. Ketogenic diet: an alternative treatment for refractory epilepsy in children. J Med Assoc Thai. 2001;84:1027-32.

13. Kossoff EH, Pyzik PL, McGrogan JR, Vining EP, Freeman JM. Efficacy of the ketogenic diet for infantile spasms. Pediatrics. 2002;109:780-3.

14. Kossoff EH, Thiele EA, Pfeifer HH, McGrogan JR, Freeman JM. Tuberous sclerosis complex and the ketogenic diet. Epilepsia. 2005;46:1684-6.

15. Maydell BV, Wyllie E, Akhtar N, Kotagal P, Powaski K, Cook K, Weinstock A, Rothner AD. Efficacy of the ketogenic diet in focal versus generalized seizures. Pediatr Neurol. 2001;25:208-12.

16. Vining EP. Clinical efficacy of the ketogenic diet. Epilepsy Res. 1999;37:181-90.

17. Keene DL. A systematic review of the use of the ketogenic diet in childhood epilepsy. Pediatr Neurol. 2006;35:1-5.

18. Lefevre F, Aronson N. Ketogenic diet for the treatment of refractory epilepsy in children: A systematic review of efficacy. Pediatrics. 2000;105:E46.

19. Levy R, Cooper P. Ketogenic diet for epilepsy. Cochrane Database Syst Rev. 2003 (3):CD001903.

20. Sinha SR, Kossoff EH. The ketogenic diet. Neurologist. 2005;11:161-70.

21. Levy RG, Cooper PN, Giri P. Ketogenic diet and other dietary treatments for epilepsy. The Cochrane database of systematic reviews. 2012;3:CD001903..

22. Neal EG, Chaffe H, Schwartz RH, Lawson MS, Edwards N, Fitzsimmons G, Whitney A, Cross JH. The ketogenic diet for the treatment of childhood epilepsy: a randomised controlled trial. Lancet Neurol. 2008;7:500-6.

23. Sharma S, Sankhyan N, Gulati S, Agarwala A. Use of the modified Atkins diet for treatment of refractory childhood epilepsy: a randomized controlled trial. Epilepsia. 2013;54:481-6.

24. Drummond MF, Sculpher MJ, Torrance GW, O'Brien BJ, Stoddart GL. Methods for the economic evaluation of health care programs. Oxford: Oxford University Press; 2005.

25. Ligtenberg G, Staal PC, Goettsch WG, Knies S. Cost-effectiveness in health care. Diemen: National Health Care Institute; 2013. 
26. de Kinderen RJ, Lambrechts DA, Postulart D, Kessels AG, Hendriksen JG, Aldenkamp AP, Evers SM, Majoie $\mathrm{MH}$. Research into the (Cost-) effectiveness of the ketogenic diet among children and adolescents with intractable epilepsy: design of a randomized controlled trial. BMC neurology. 2011;11:10.

27. EuroQol G. EuroQol--a new facility for the measurement of health-related quality of life. The EuroQol Group. Health Policy. 1990;16:199-208.

28. Wille N, Badia X, Bonsel G, Burström K, Cavrini G, Devlin N, Egmar AC, Greiner W, Gusi N, Herdman M, Jelsma J, Kind P, Scalone L, Ravens-Sieberer U. Development of the EQ-5D-Y: a child-friendly version of the EQ-5D. Qual Life Res. 2010;19:875-86.

29. Lamers LM, McDonnell J, Stalmeier PF, Krabbe PF, Busschbach JJ. The Dutch tariff: results and arguments for an effective design for national EQ-5D valuation studies. Health Econ. 2006;15: 1121-32..

30. Fekkes M, Theunissen NC, Brugman E, Veen S, Verrips EG, Koopman HM, Vogels T, Wit JM, VerlooveVanhorick SP. Development and psychometric evaluation of the TAPQOL: a health-related quality of life instrument for 1-5-year-old children. Qual Life Res. 2000;9:961-72.

31. Vogels T, Verrips GH, Koopman HM. TACQOL manual. Parent Form and Child Form. Leiden: Leiden Center for Child Health and Pediatrics LUMC-TNO. 2000.

32. Goossens ME, Rutten-van Molken MP, Vlaeyen JW, van der Linden SM. The cost diary: a method to measure direct and indirect costs in cost-effectiveness research. J Clin Epidemiol. 2000;53:688-95.

33. Hakkaart - van Roijen L, Tan SS, Bouwmans CAM. Handleiding voor kostenonderzoek. Amstelveen: CVZ; 2010.

34. Council-for-Public-Health-and-Health-Care. Sensible and sustainable care (in Dutch). Zoetermeer: Council for Public Health and Health Care 2006.

35. Salomon JA, Vos T, Hogan DR, Gagnon M, Naghavi M, Mokdad A, Begum N, Shah R, Karyana M, Kosen S, Farje MR, Moncada G, Dutta A, Sazawal S, Dyer A, Seiler J, Aboyans V, Baker L, Baxter A, Benjamin EJ, Bhalla K, Bin Abdulhak A, Blyth F, Bourne R, Braithwaite T, Brooks P, Brugha TS, Bryan-Hancock C, Buchbinder R, Burney P, Calabria B, Chen H, Chugh SS, Cooley R, Criqui MH, Cross M, Dabhadkar KC, Dahodwala N, Davis A, Degenhardt L, Díaz-Torné C, Dorsey ER, Driscoll T, Edmond K, Elbaz A, Ezzati M, Feigin V, Ferri CP, Flaxman AD, Flood L, Fransen M, Fuse K, Gabbe BJ, Gillum RF, Haagsma J, Harrison JE, Havmoeller R, Hay RJ, Hel-Baqui A, Hoek HW, Hoffman H, Hogeland E, Hoy D, Jarvis D, Karthikeyan G, Knowlton LM, Lathlean T, Leasher JL, Lim SS, Lipshultz SE, Lopez AD, Lozano R, Lyons R, Malekzadeh R, Marcenes W, March L, Margolis DJ, McGill N, McGrath J, Mensah GA, Meyer AC, Michaud C, Moran A, Mori R, Murdoch ME, Naldi L, Newton CR, Norman R, Omer SB, Osborne R, Pearce N, Perez-Ruiz F, Perico N, Pesudovs K, Phillips D, Pourmalek F, Prince M, Rehm JT, Remuzzi G, Richardson K, Room R, Saha S, Sampson U, Sanchez-Riera L, Segui-Gomez M, Shahraz S, Shibuya K, Singh D, Sliwa K, Smith E, Soerjomataram I, Steiner T, Stolk WA, Stovner LJ, Sudfeld C, Taylor HR, Tleyjeh IM, van der Werf MJ, Watson WL, Weatherall DJ, Weintraub R, Weisskopf MG, Whiteford H, Wilkinson JD, Woolf AD, Zheng ZJ, Murray CJ, Jonas JB. Common values in assessing health outcomes from disease and injury: disability weights measurement study for the Global Burden of Disease Study 2010. Lancet. 2012;380:2129-43.

36. NICE. The guidelines manual. Process and methods gides. National Institute for Health and Care Excellence. 2012.

37. Campo SF, Sharpton WR, Thompson B, Sexton D. Measurement characteristics of the Quality of Life Index when used with adults who have severe mental retardation. American journal of mental retardation : AJMR. 1996;100:546-50.

38. de Kinderen, RJ, Postulart D, ALdenkamp AP, Evers SM, Lambrechts DA, Louw AJ, Majoie HJ, Grutters $J P$. Cost-effectiveness of the ketogenic diet and vagus nerve stimulation for the treatment of children with intractable epilepsy. Epilepsy Res. 2015;110:119-131.

39. Mackay MT, Bicknell-Royle J, Nation J, Humphrey M, Harvey AS. The ketogenic diet in refractory childhood epilepsy. Journal of paediatrics and child health. 2005;41:353-7. 


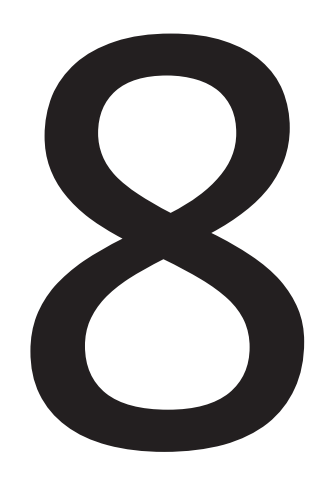

\section{Cost-effectiveness of the ketogenic diet and vagus} nerve stimulation for the treatment of children with intractable epilepsy

RJA de Kinderen, D Postulart, AP Aldenkamp, SMAA Evers, DAJE Lambrechts, AJA de Louw, HJM Majoie, JPC Grutters Epilepsy Research 2015;110:119-131 


\section{Abstract}

\section{Purpose}

The objective of this study was to estimate the expected cost-utility and cost-effectiveness of the Ketogenic Diet (KD), Vague Nerve Stimulation (VNS) and Care As Usual (CAU), using a decision analytic model with a 5-year time horizon.

\section{Methods}

A Markov decision analytical model was constructed to estimate the incremental costs, qualityadjusted life years (QALYs) and successfully treated patient (i.e. 50\% or more seizure reduction) of the treatment strategies KD, VNS and CAU, from a health care perspective. The base case considered children with intractable epilepsy (i.e. two or more antiepileptic drugs had failed) aged between 1 and 18 years. Data were derived from literature and expert meetings. Deterministic and probabilistic sensitivity analyses were performed.

\section{Results}

Our results suggest that KD is more effective and less costly, and thus cost-effective compared with VNS, after 12 months. However, compared to CAU, neither KD nor VNS are cost-effective options, they are both more effective but also more expensive (€346,899 and €641,068 per QALY, respectively). At 5 years, VNS is cost-effective compared with KD and CAU (€11,378 and $€ 68,489$ per QALY, respectively) and has a $51 \%$ probability of being cost-effective at a ceiling ratio of $€ 80,000$ per QALY.

\section{Conclusions}

Our results suggest that on average the benefits of KD and VNS fail to outweigh the costs of the therapies. However, these treatment options should not be ignored in the treatment for intractable epilepsy in individual or specific groups of patients. There is a great need for high quality comparative studies with large patient samples which allow for subgroup analyses, longterm follow-up periods and outcome measures that measure effects beyond seizure frequency (e.g. quality of life). When this new evidence becomes available, reassessment of the costeffectiveness of KD and VNS in children with intractable epilepsy should be carried out. 


\section{Introduction}

Epileptic seizures are treatable with antiepileptic drugs (AEDs) in the majority of patients. About $30 \%$ of patients suffer from intractable epilepsy, indicating that their seizures are still uncontrolled after taking two or more different AEDs. ${ }^{1}$ Some of these patients are eligible for resective surgery, where the area of the brain responsible for seizures is surgically removed. If resective surgery fails or is not an option, children with intractable epilepsy in particular can potentially benefit from a Ketogenic Diet (KD). ${ }^{2}$ The KD is a high-fat, low carbohydrate diet that imitates the metabolic state of fasting while remaining normocaloric. Body energy requirements while on the KD are met by lipolysis and B-oxidation of fatty acids rather than by the breakdown of carbohydrates. Another treatment option for children with intractable epilepsy could be vague nerve stimulation (VNS). VNS is a pacemaker-like device that is implanted subcutaneously into the upper part of the chest with a connecting wire running from the stimulator to an electrode that is attached to the vagus nerve. ${ }^{3}$ Once the stimulator is activated, electrical pulses will be generated at regular intervals depending on the anticonvulsant effect and on the patients' tolerance.

Although the exact anticonvulsant working mechanisms are still unknown ${ }^{3,4}$, the beneficial effects of both the KD and VNS are separately reported. ${ }^{4,5}$

Direct comparisons of the KD and VNS are, however, lacking, which makes it difficult to determine the most optimal treatment. In addition, due to scarcity of resources, it is increasingly important to examine whether benefits outweigh costs. The use of decision modeling in economic evaluation is a powerful tool for investigating this trade-off between costs and effects of comparative treatments. Modeling allows the synthesis of all available evidence from different sources. Modeling also makes it possible to include long-term costs and benefits in the analysis. ${ }^{7}$ This is important because the impact of epilepsy (treatments) on patients' lives, seizures, and costs extends beyond the study period of most clinical studies.

The primary aim of the present study is to use a three-arm decision analytical model to evaluate the cost-effectiveness of the KD and VNS as compared to Care As Usual $(\mathrm{CAU})$ in children aged between $1-18$ years with intractable epilepsy.

\section{Methods}

\section{Model description}

A probabilistic Markov decision-analytical model was developed to estimate the costeffectiveness and cost-utility of the KD, VNS and CAU as a treatment for a hypothetical cohort of children with intractable epilepsy, uncontrolled by two or more AEDs and ineligible for resective surgery. CAU means that the child continues to take his or her AEDs and no changes will be made to the AED treatment. 
The model was built in Microsoft Excel 2010 following established economic evaluation state-transition modeling guidelines. ${ }^{6}$ Costs and effects were modeled with a time horizon of 5 years.

Figure 8.1 is a graphical representation of the model. In the first cycle, the hypothetical cohort started on KD, VNS or stayed on CAU. After three months, patients may have switched from KD or VNS to CAU (due to e.g. severe adverse events, difficulty of treatment or other reasons) or have died. In case of withdrawal from treatment, patients on KD would normalize their diet and VNS-treated patients would have their VNS switched off or removed surgically. After the first cycle, patients entered one of the health states: 1) seizure-free, 2) improvement (50\% or more seizure reduction), and 3 ) no improvement (less than $50 \%$ seizure reduction). The absorbing state was 'death', either due to Sudden Unexpected Death in Epilepsy (SUDEP) or death from other causes. In the model, patients move between these mutually exclusive health states according to transition probabilities.

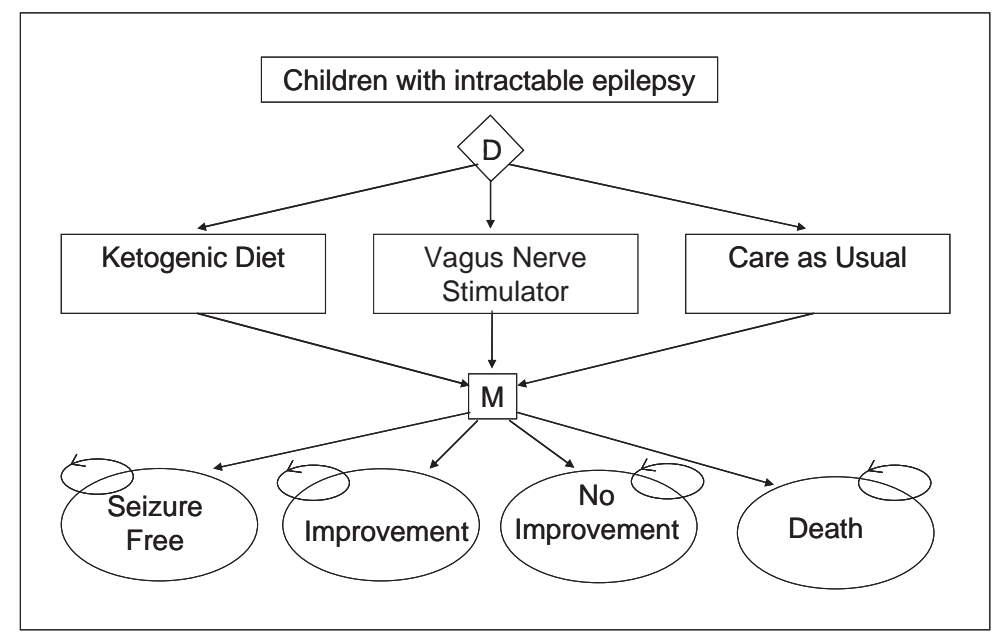

Figure 8.1 Graphical representation Markov Model.

D, Decision note; M; Markov note

\section{Validation}

In order to validate the model ${ }^{8}$ and to retrieve background information needed to further develop the model, we organized two expert meetings. In the first session, the decision problem was outlined and assumptions required to determine the structure of the model were generated. In the second session, the model and its inputs were validated. The foremost criterion for expert selection was the extent of their expertise and experience with intractable epilepsy in children and/or research experience in the field of efficacy and cost-effectiveness. ${ }^{9}$ During the first meeting three neurologists $(\mathrm{DL}, \mathrm{AL}$, and $\mathrm{MM})$, one behavioral scientist (AA) and one HTA expert (RdK) participated. During the second meeting, three neurologists ( $D L, A L$, and $M M$ ) and three HTA experts (JG, DP and RdK) took part in the discussion. 


\section{Transition probabilities}

An overview of the transition probabilities and their data sources is presented in Table 8.1. An extensive literature search identified two randomized controlled trials (RCTs) in which KD was compared with $C A U,{ }^{10,11}$ and three RCTs that compared VNS with CAU. ${ }^{12-14}$ No RCTs comparing KD with VNS were found. Since two VNS studies, ${ }^{14,15}$ partially reported on the same patients, we combined these studies to correct for double counting. These RCTs and their long-term follow-up studies ${ }^{16-19}$ were used to estimate probabilities regarding efficacy and safety. Pooled proportion analyses were used to calculate weighted average probabilities based on a random effects model. Data from the control arms of both KD RCTs ${ }^{10,11}$ were used to estimate probabilities regarding effectiveness for CAU. We decided not to include the data of control arms of the VNS RCTs because of the expected placebo effect, as the control group in these trials did receive VNS implantation surgery and stimulation although the settings were not thought to have any therapeutic value.

Table 8.1 List of input parameters for the transition probabilities.

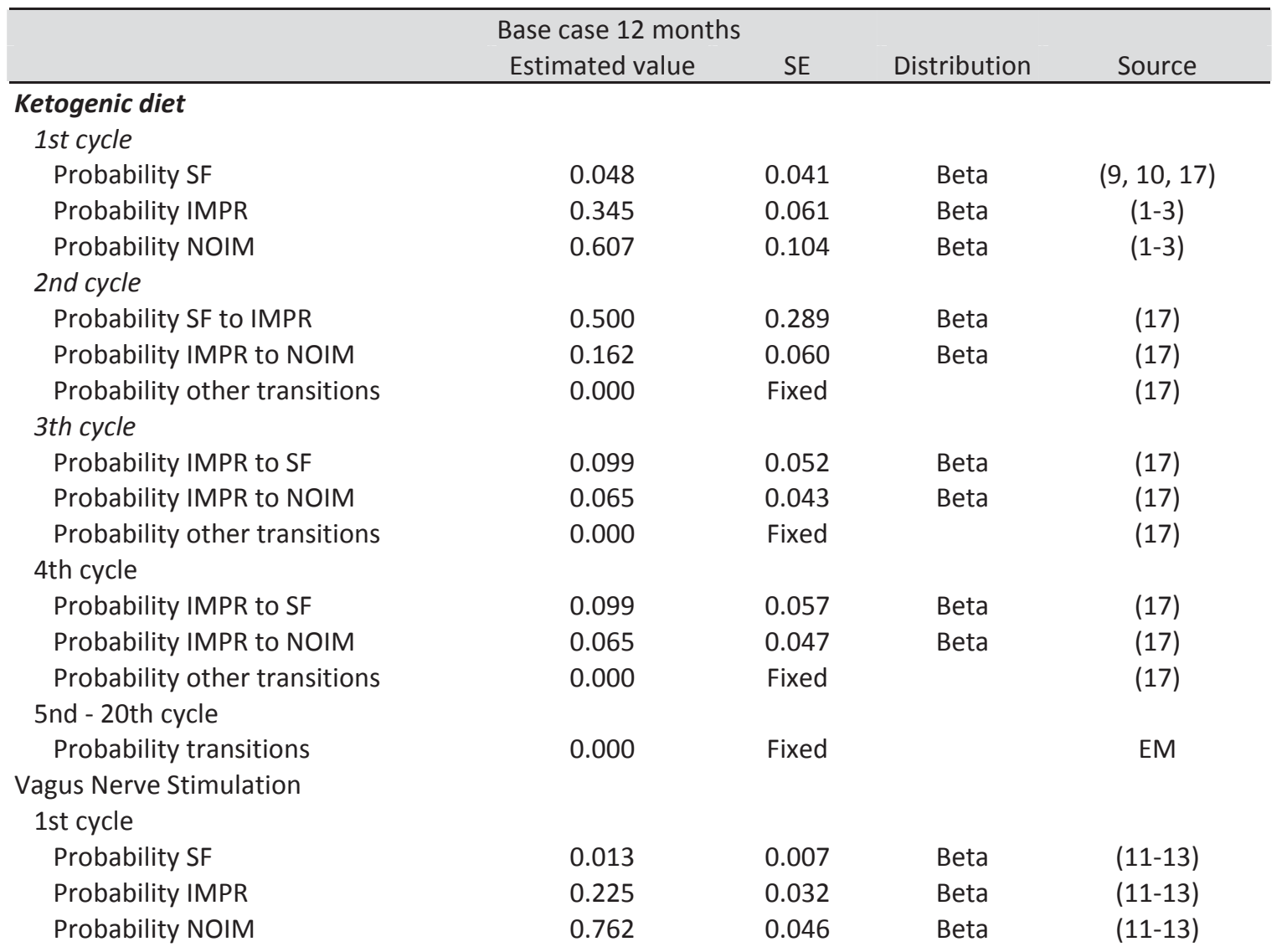


Table 8.1 (continued)

\begin{tabular}{|c|c|c|c|c|}
\hline & Base case 12 mon & & & \\
\hline & Estimated value & SE & Distribution & Source \\
\hline 2nd cycle & & & & \\
\hline Probability SF to IMPR & 0.006 & 0.005 & Beta & $(12,15,16,18)$ \\
\hline Probability SF to NOIM & 0.003 & 0.004 & Beta & $(12,15,16,18)$ \\
\hline Probability IMPR to SF & 0.003 & 0.003 & Beta & $(12,15,16,18)$ \\
\hline Probability IMPR to NOIM & 0.008 & 0.010 & Beta & $(12,15,16,18)$ \\
\hline Probability NOIM to SF & 0.015 & 0.012 & Beta & $(12,15,16,18)$ \\
\hline Probability NOIM to IMPR & 0.015 & 0.012 & Beta & $(12,15,16,18)$ \\
\hline Probability other transitions & 0.000 & Fixed & & $(12,15,16,18)$ \\
\hline 3th cycle & & & & \\
\hline Probability IMPR to SF & 0.011 & 0.010 & Beta & $(12,15,16,18)$ \\
\hline Probability IMPR to NOIM & 0.011 & 0.010 & Beta & $(12,15,16,18)$ \\
\hline Probability NOIM to SF & 0.006 & 0.005 & Beta & $(12,15,16,18)$ \\
\hline Probability NOIM to IMPR & 0.034 & 0.021 & Beta & $(12,15,16,18)$ \\
\hline Probability other transitions & 0.000 & Fixed & & $(12,15,16,18)$ \\
\hline 4th cycle & & & & \\
\hline Probability IMPR to SF & 0.011 & 0.010 & Beta & $(15,16,18)$ \\
\hline Probability IMPR to NOIM & 0.011 & 0.010 & Beta & $(15,16,18)$ \\
\hline Probability NOIM to SF & 0.006 & 0.005 & Beta & $(15,16,18)$ \\
\hline Probability NOIM to IMPR & 0.009 & 0.008 & Beta & $(15,16,18)$ \\
\hline Probability other transitions & 0.000 & Fixed & & $(15,16,18)$ \\
\hline 5th cycle & & & & \\
\hline Probability NOIM to SF & 0.005 & 0.002 & Beta & $(15,16)$ \\
\hline Probability NOIM to IMPR & 0.006 & 0.019 & Beta & $(15,16)$ \\
\hline Probability other transitions & 0.000 & Fixed & & $(15,16)$ \\
\hline 6th cycle & & & & \\
\hline Probability NOIM to IMPR & 0.009 & 0.008 & Beta & (16) \\
\hline Probability other transitions & 0.000 & Fixed & & (16) \\
\hline 7nd - 20th cycle & & & & \\
\hline Probability transitions & 0.000 & Fixed & & EM \\
\hline Care as Usual & & & & \\
\hline 1st cycle & & & & \\
\hline Probability SF & 0.008 & 0.008 & Beta & (9) \\
\hline Probability IMPR & 0.075 & 0.028 & Beta & (9) \\
\hline Probability NOIM & 0.917 & 0.028 & Beta & (9) \\
\hline 2nd - 20th cycle & & & & \\
\hline Probability transitions & 0.000 & Fixed & & EM \\
\hline Withdrawal & & & & \\
\hline Probability withdrawal KD & 0.128 & 0.025 & Beta & $(9,10,17)$ \\
\hline - due to side effects & 0.536 & 0.153 & Beta & $(9,10,17)$ \\
\hline - due to other reasons & 0.464 & 0.153 & Beta & $(9,10,17)$ \\
\hline Probability withdrawal VNS & 0.016 & 0.007 & Beta & $(11,14)$ \\
\hline - due to side effects & 0.709 & 0.152 & Beta & $(11,14)$ \\
\hline - due to other reasons & 0.291 & 0.152 & Beta & $(11,14)$ \\
\hline Probability withdrawal + removal VNS & 0.008 & Fixed & & $(11,14)$ \\
\hline Probability withdrawal CAU & 0.000 & Fixed & & (9) \\
\hline
\end{tabular}

SE: standard error; SF: seizure Free; IMPR:, improvement (e.g. 50\% or more seizure reduction); NOIM: no improvement (e.g. less than $50 \%$ seizure reduction); KD: ketogenic diet; VNS: vagus nerve stimulation; CAU: care as usual; EM: expert meeting 
Annual age-specific all-cause mortality rates were based on Dutch life tables ${ }^{20}$ and transformed into three-month mortality rates. SUDEP rates were added to the allcause mortality rates, based on an extensive literature review by Shorvon and Tomson (2011). ${ }^{21}$ Complications regarding VNS implantation were included in the model in cases when removal of the device was necessary. Side effects due to antiepileptic drugs were not included in the model, yet assumed to be similar for all patients in each treatment arm. Other complications, such as gastrointestinal complaints and hoarseness, were not incorporated in the model; there are many, with generally a limited or short-term impact on quality of life or costs. Patients on KD would follow the dietary treatment for a maximum of 24 months, after this period they were treated with CAU. We assumed that a responder remains a responder and a nonresponder remains a non-responder for the rest of the study period (i.e. patients do not switch between health states after 24 months).

\section{Effects}

Quality-adjusted life years (QALYs) were the primary measure of effect. The combination of utility scores and life expectancy allows for the calculation of QALYs, and cost per QALY ratios. Utility was considered as a single index on a scale from 0 (representing death) to 1 (representing full health). A literature search identified four studies that computed utility values for an appropriate population of subjects with epilepsy. ${ }^{22-25}$ In the second expert meeting we chose to use the utility values of Messori et al., $(1998)^{22}$, as the health states described in their paper best matched the health states in our model (Table 8.2). A secondary outcome measure was the number of successfully treated patients, defined as patients with seizure reduction of $50 \%$ or more.

Following the Dutch guidelines for pharmaco-economic research, an annual discount rate of $1.5 \%$ was applied to the effects. ${ }^{26}$

\section{Costs}

Costs were calculated in Euros, from a health care perspective and were converted (if necessary) to the price year 2013 (Table 8.2). Estimates of healthcare resource usage were obtained in the expert meetings, as most of them were not available in the literature. Consensus was reached on drug use, the frequency of contacts with the nurse practitioner, neurologists and other specialists related to patient monitoring and switching treatments, and treatment of adverse effects. Epilepsy related hospitalizations were obtained from the literature. ${ }^{27}$ The initiation costs of KD were based on the standardized protocol of the tertiary epilepsy center Kempenhaeghe in The Netherlands. These costs consisted of a 5-day admission to the epilepsy center, visits of the neurologist, pediatrician, dietician and epilepsy nurse, and laboratory costs. Costs related to the KD were vitamin and diet supplements, and keto sticks. Furthermore, $80 \%$ of the patients would receive the Multi Chain Triglyceride (MCT) 
diet, $15 \%$ the classical diet and $5 \%$ diet via tube feeding. VNS implantation costs consisted of a 2-day admission to an academic hospital, surgery (operating room, neurosurgeon, and anesthesia), device (pulse generator, wires and magnet) and visits to the epilepsy nurse. Where possible, unit costs were based on standardized costs from the Dutch guidelines for cost research. ${ }^{28}$ Cost of antiepileptic drugs and the cost of side effects due to antiepileptic drugs were not taken into account; they were assumed to be equal in all three treatment arms. Future costs were discounted to their present value by a rate of $4 \% .^{26}$

Table 8.2 List of input parameters for the costs and effects per cycle.

\begin{tabular}{|c|c|c|c|c|}
\hline & Estimated value & Range & Distribution & Source \\
\hline \multicolumn{5}{|l|}{ Resource use (volumes) } \\
\hline \multicolumn{5}{|l|}{ Seizure free } \\
\hline - number of neurologist visits & 0.25 & $0.0-0.50$ & Beta PERT & EM \\
\hline - number of seizure related hospitalizations & 0.275 & $0.0-0.50$ & Beta PERT & $(26)$ \\
\hline \multicolumn{5}{|l|}{ Improvement } \\
\hline - number of neurologist visits & 0.5 & $0.25-0.75$ & Beta PERT & EM \\
\hline - number of hospitalizations & 0.625 & $0.50-0.75$ & Beta PERT & (26) \\
\hline \multicolumn{5}{|l|}{ No Improvement } \\
\hline - number of neurologist visits & 1.0 & $0.75-1.25$ & Beta PERT & EM \\
\hline - number of hospitalizations & 1.65 & $1.0-2.0$ & Beta PERT & (26) \\
\hline \multicolumn{5}{|l|}{ Unit costs $(€)$} \\
\hline Initiation KD & $4,542.22$ & Fixed & & EM, PC, (27) \\
\hline MCT diet & $2,008.71$ & Fixed & & EM, PC, (27) \\
\hline Tube diet & $2,666.70$ & Fixed & & EM, PC, (27) \\
\hline Medical vitamin supplements & 105.55 & $95-116$ & Beta PERT & PC \\
\hline Regular vitamin supplements & 35.31 & $19.80-50.40$ & Beta PERT & PC \\
\hline Keto sticks & 20.68 & $11.88-26.98$ & Beta PERT & PC \\
\hline Outpatient visit to specialist & 78.12 & Fixed & & EM, PC, (27) \\
\hline Visit to epilepsy nurse & 109.33 & Fixed & & PC \\
\hline Hospitalization per day & 497.67 & Fixed & & EM, PC, (27) \\
\hline VNS implantation & $16,889.98$ & Fixed & & $\mathrm{EM}, \mathrm{PC},(27)$ \\
\hline Removal VNS & $2,700.00$ & Fixed & & PC \\
\hline \multicolumn{5}{|l|}{ Utilities per Health state } \\
\hline Seizure free & 0.935 & $0.754-1.00$ & Beta PERT & $(21,23)$ \\
\hline Improvement & 0.790 & $0.51-1.00$ & Beta PERT & $(21,23)$ \\
\hline No Improvement & 0.660 & $0.461-0.78$ & Beta PERT & $(21,23)$ \\
\hline Withdrawal & 0.400 & $0.32-0.50$ & Beta PERT & $(21,23)$ \\
\hline \multicolumn{5}{|l|}{ SUDEP mortality per Health state } \\
\hline Seizure free & 0.00028 & Fixed & & (20) \\
\hline Improvement & 0.00148 & Fixed & & (20) \\
\hline No Improvement & 0.00233 & Fixed & & (20) \\
\hline
\end{tabular}

EM: expert meeting; PC: personal communication; KD: ketogenic diet; $\mathrm{MCT}$ : multi chain triglyceride; VNS: vagus nerve stimulation; SUDEP: sudden unexpected death in epilepsy.

\section{Analysis}

Costs and effects were modeled with a time horizon of 12 months and at 5 years. Incremental cost-effectiveness ratios (ICERs) were calculated by dividing the incremental costs by the incremental QALYs. The ICER represents the cost of an 
additional QALY gained and was used to estimate the cost-effectiveness of KD and VNS as opposed to CAU. Subsequently, the ICER of KD opposed to VNS was calculated. The ICERs were presented on a cost-effectiveness plane, which is divided into four quadrants. The south-east quadrant represents the instances where the intervention is more effective and less costly than the comparators (dominates), with the opposite situation in the north-west quadrant, representing interventions that are more costly and less effective than comparators (dominated). The north-east quadrant represents interventions that are more costly yet more effective, while the south-west quadrant represents less effective and less costly interventions. ${ }^{29} \mathrm{~A}$ treatment is deemed costeffective when it is dominant or when the costs per QALY are below a specified ceiling ratio. In The Netherlands, an informal ceiling ratio between approximately $€ 20,000$ and $€ 80,000$ per QALY exist, depending on the burden of disease. ${ }^{30}$ Furthermore, Cost-Effectiveness Acceptability Curves (CEACs) were calculated. ${ }^{29}$ CEACs show the probability that a treatment is cost-effective, given different ceiling ratios. Finally, the number of responding patients and incremental cost per responding patient were calculated.

\section{Sensitivity analysis}

Uncertainty surrounding input parameter estimates, such as efficacy, utilities and costs, is characterized by the limitations or absence of evidence available to inform input parameters. Probabilistic sensitivity analysis was used to capture the uncertainty in the input parameters. This means that we assigned distributions to the model parameters. ${ }^{31}$ Uncertainty in efficacy transition probabilities was expressed as Betadistributions, while uncertainties in cost data and utility values were expressed as Beta PERT distributions.

A distribution of the incremental costs and benefits was determined by sampling a value from each input parameter distribution, calculating the results with the model, then repeating this process 1000 times. The results were presented with a point estimate and $95 \%$ confidence intervals, as well as a joint-distribution of incremental costs and QALYs on a cost-effectiveness plane, illustrating the uncertainty surrounding the estimates of incremental costs and QALYs.

Deterministic sensitivity analysis was used to investigate the impact of the cost difference between different variants of the KD. Although Neal et al. (2009) ${ }^{18}$ showed no difference in effects between the classical KD variant and the MCT KD variant, there is however, a cost difference between these variants. The base case analysis assumes that $80 \%$ of the patients will be treated with the MCT variant and $5 \%$ via tube feeding. As the number of patients on the MCT is highly dependent on the institute's (or country) specific protocol or guidelines, we investigated this cost difference in a sensitivity analysis in which we assumed that $100 \%$ of the patients will be treated with the classical variant of the KD. Additionally, we lowered the intervention costs of KD by assuming that no hospitalization is necessary for the initiation of the diet. 


\section{Results}

\section{Analysis at 12 months}

For children with intractable epilepsy, 34\% of the patients on KD were responders (i.e. $50 \%$ or more seizure reduction), resulting on average in 0.693 QALYs per patient. The expected health care costs were $€ 14,036$ per patient over 12 months. VNS therapy yielded less responders (27\%) and less QALYs (0.688) and was more expensive $(€ 19,922)$. Comparing KD with VNS, KD dominated VNS in terms of cost per QALY and per responder, as KD is less costly and more effective. CAU was the least effective (0.662 QALYs and 8\% responders) and also the least expensive therapy $(€ 3,306)$. Comparing KD with CAU resulted in an ICER of $€ 346,899$ per QALY gained (i.e. more effects at higher costs). The ICERS are presented in Table 8.3.

Table 8.3 Results of the cost-effectiveness analyses.

\begin{tabular}{|c|c|c|c|c|c|c|}
\hline \multirow{2}{*}{ At 12 months } & \multicolumn{2}{|c|}{ Expected costs in $€(95 \% \mathrm{Cl})$} & \multicolumn{2}{|c|}{ Expected QALYs $(95 \% \mathrm{Cl})$} & \multicolumn{2}{|c|}{ ICER (€ per QALY) } \\
\hline & & & & & & \\
\hline KD & 14,036 & $(13,310-14,735)$ & 0.693 & $(0.579-0.747)$ & KD-VNS & Dominant \\
\hline VNS & 19,922 & $(19,391-20,259)$ & 0.688 & $(0.571-0.742)$ & KD-CAU & 346,899 \\
\hline CAU & 3,306 & $(2,405-3,851)$ & 0.662 & $(0.536-0.710)$ & VNS-CAU & 641,068 \\
\hline \multicolumn{7}{|c|}{ At 5 years } \\
\hline KD & 30,935 & $(27,610-33,347)$ & 3.338 & $(2.823-3.612)$ & KD-VNS & 11,378 \\
\hline VNS & 31,581 & $(29,233-33,356)$ & 3.395 & $(2.876-3.747)$ & KD-CAU & 86,025 \\
\hline CAU & 15,029 & $(11,155-17,389)$ & 3.153 & $(2.604-3.372)$ & VNS-CAU & 68,489 \\
\hline
\end{tabular}

QALYs: quality adjusted life years; ICER: incremental cost-effectiveness ratio; KD: ketogenic diet; VNS: vagus nerve stimulator; CAU: care as usual.

\section{Analysis at 5 years}

After 5 years, VNS therapy yielded the most QALYs (3.40) and the highest percentage responders (42\%). The expected health care costs were €31,581 per patient. KD yielded fewer QALYs (3.34), less responders (30\%) and was slightly less expensive (€30,935). The ICER of VNS compared to KD is €11,378 per QALY gained (Table 8.3). CAU was the least effective (3.15 QALYs and $8 \%$ responders) and was the least expensive $(€ 15,029)$. VNS compared to CAU resulted in an ICER of $€ 68,489$ per QALY gained. 
A

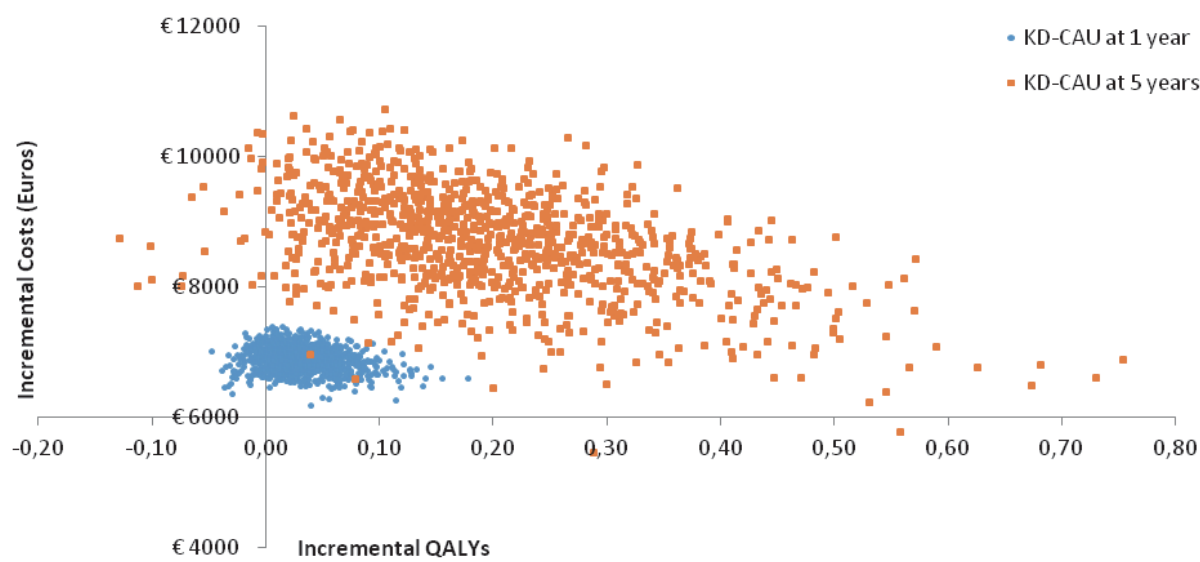

B

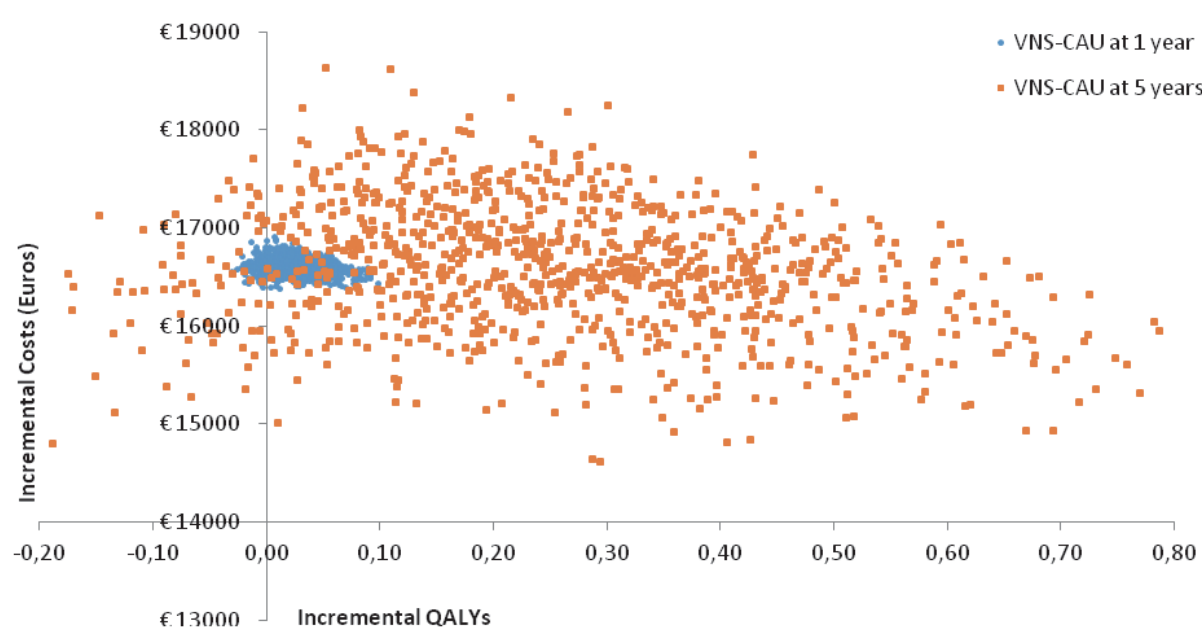

C

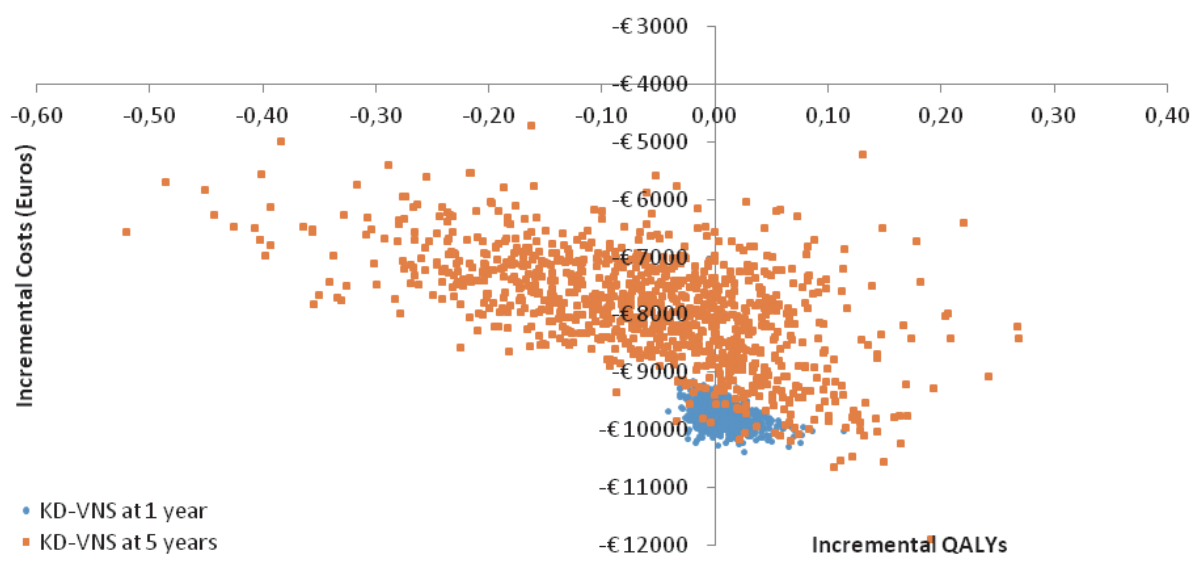

Figure 8.2 Cost-effectiveness planes for (A) KD compared to CAU at 1 and 5 year, (B) VNS compared to CAU at 1 and 5 year, (C) KD compared to VNS at 1 and 5 year. ICER, Incremental Cost Effectiveness Ratio; QALYs, Quality Adjusted Life Years; KD, Ketogenic Diet; CAU, Care As Usual; VNS, Vagus Nerve Stimulation. 
A

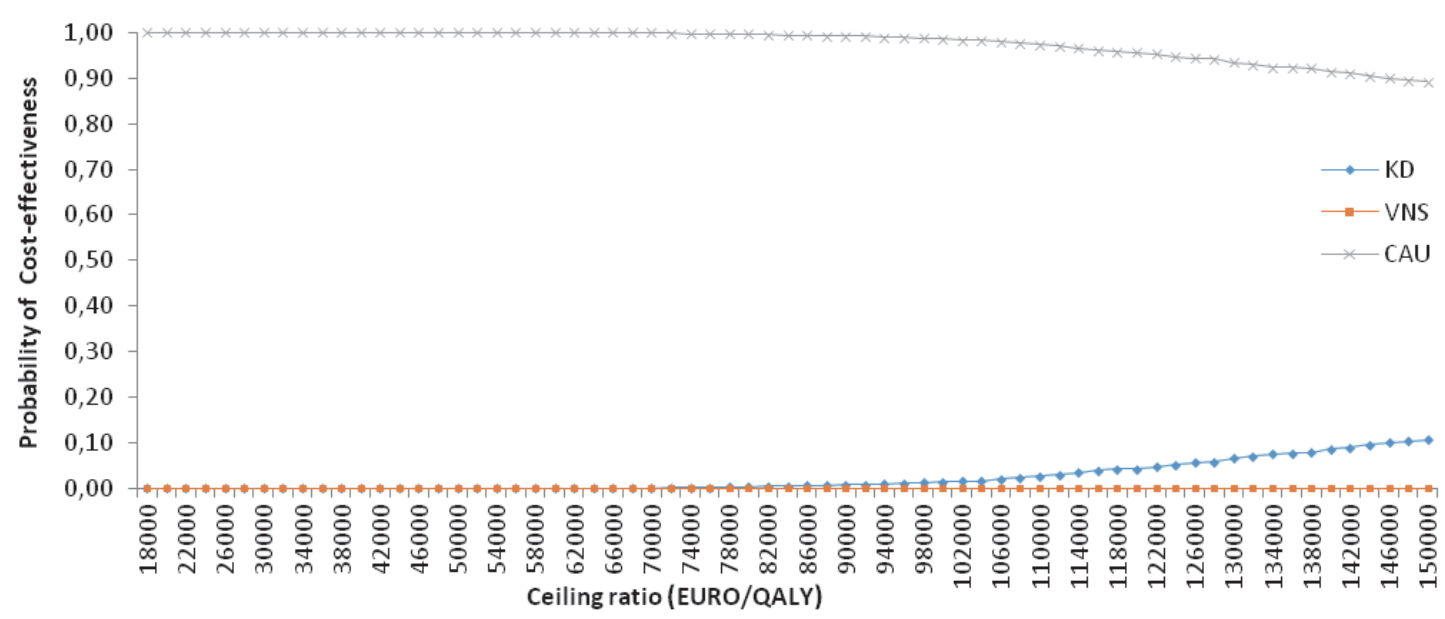

B
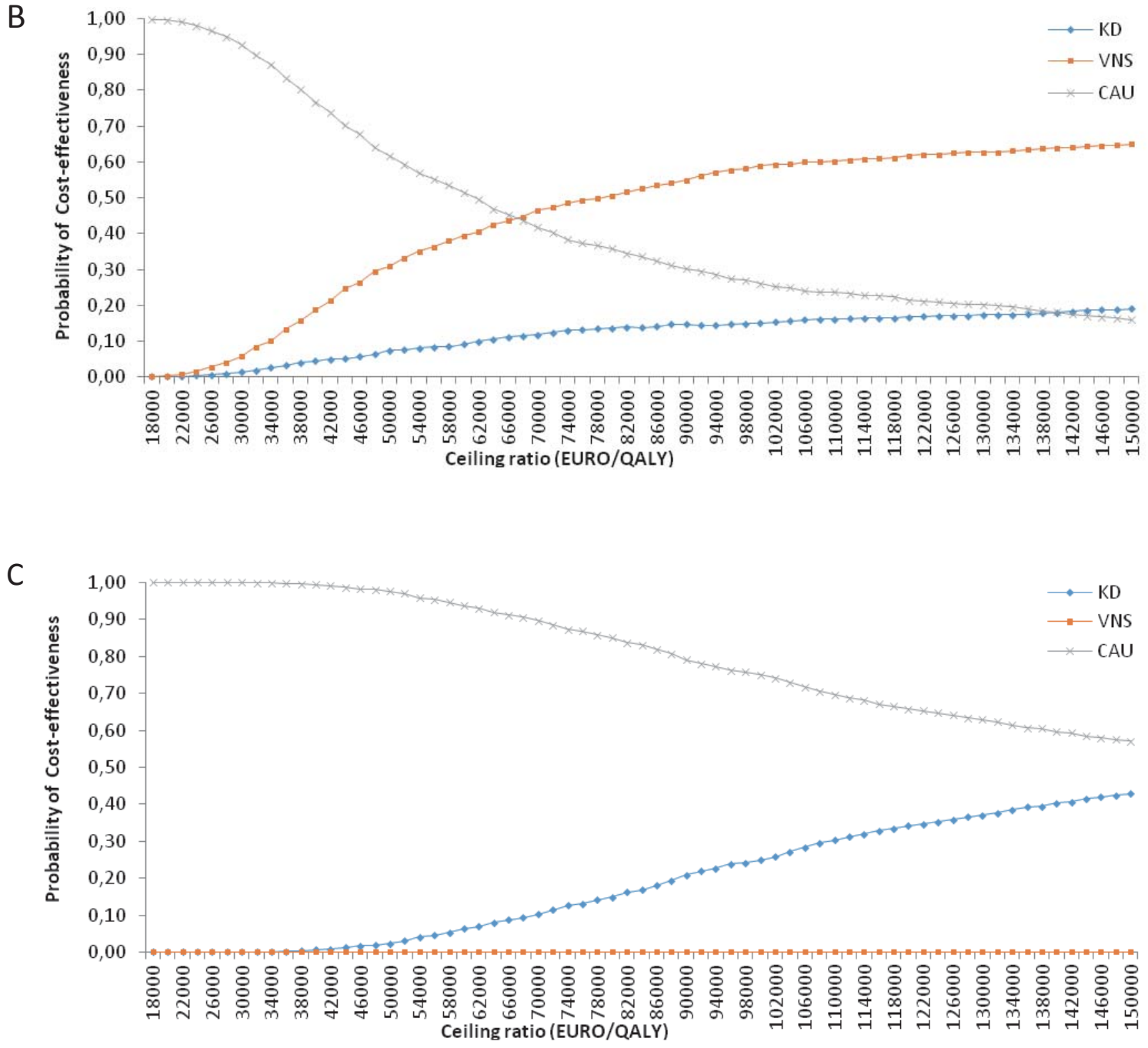


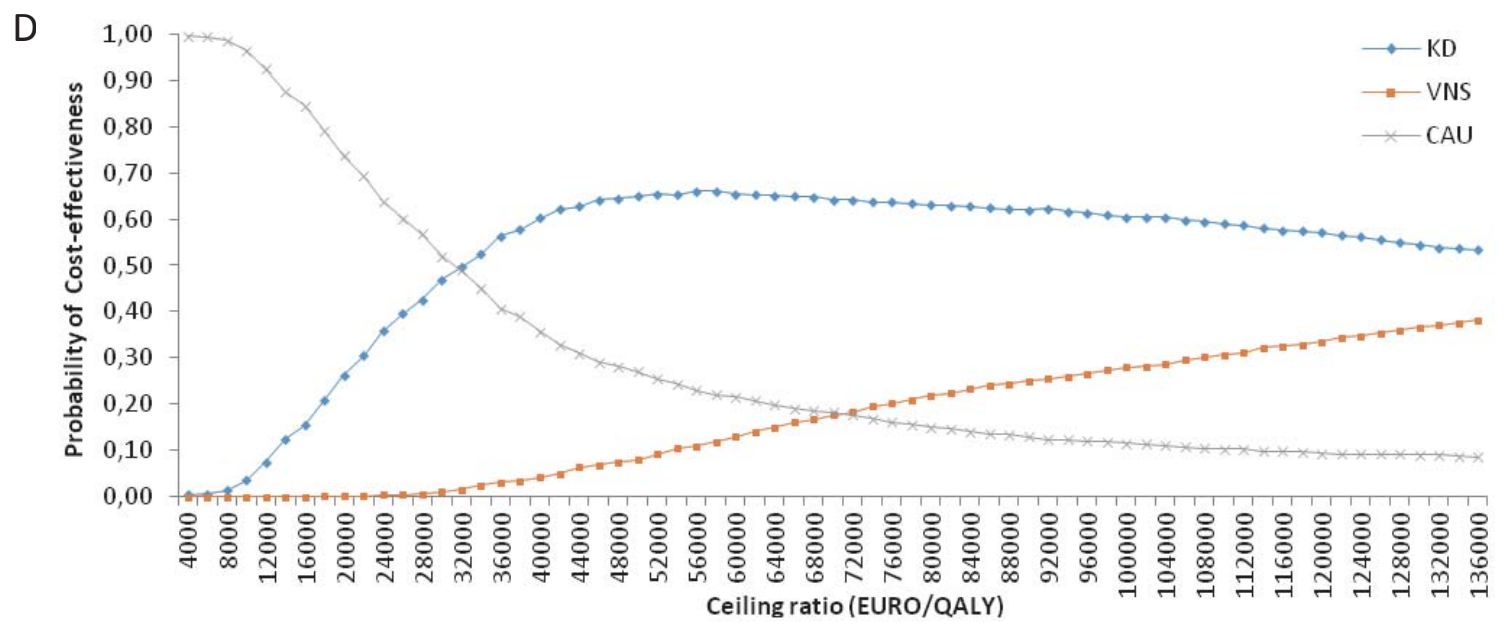

Figure 8.3 Cost-Effectiveness Acceptability Curves (CEACs) for (A) analysis at 12 months, (B) analysis at 5 years, (C) sensitivity analysis with $100 \%$ of patients on the classical KD variant (no MCT) and no hospitalization at 12 months, and (D) sensitivity analysis with $100 \%$ of patients on the classical KD variant (no MCT) and no hospitalization at 5 years. Quality Adjusted Life Years; KD, Ketogenic Diet; CAU, Care As Usual; VNS, Vagus Nerve Stimulation; MCT, Multi Chain Triglyceride.

\section{Sensitivity analysis}

Figure 8.2 shows the results of the probabilistic sensitivity analyses. The scatterplots display the uncertainty surrounding the ICERs, the dots representing the probabilistic sensitivity analysis at 12 months and the cubes at 5 years. At 12 months and 5 years, CAU had a $100 \%$ probability of being cost-effective at a ceiling ratio of $€ 20,000$ (Figure $8.3 \mathrm{~A}$ and B). At a ceiling ratio of $€ 80,000$, at 12 months CAU still had a $100 \%$ probability of being cost-effective (Figure 8.3A), and at 5 years VNS had the highest probability (51\%) of being cost-effective, followed by CAU (36\%) and KD (14\%) (Figure 8.3B).

In a deterministic sensitivity analysis, we estimated the costs of the KD when all of the KD patients were on the classical variant instead of the MCT variant. The total health care costs of classical KD at 12 months are $€ 8,297$. Additionally, we decreased the costs of the classical KD by assuming that no hospitalization was necessary to initiate the diet, resulting in a total health care costs of $€ 6,550$ at 12 months. For a ceiling ratio of $€ 20,000$ and $€ 80,000$ CAU still has the highest probability of being costeffective (100\% resp. $91 \%$ ), followed by classical KD (0\% resp. $9 \%$ ) and VNS (0\% resp. $0 \%$ ) (Figure 8.3C). The total health care cost of KD at 5 years when $100 \%$ of the patients uses the classical KD, are $€ 18,550$. For a ceiling ratio of $€ 20,000$, again CAU has the highest probability of being cost-effective (74\%), followed by KD (26\%) and VNS (0\%) (Figure 8.3D). For a ceiling ratio of $€ 80,000$ the probability of the classical KD being cost-effective is $63 \%$, followed by VNS (22\%) and CAU (15\%). 


\section{Discussion}

In the analysis at 12 months, neither KD nor VNS are cost-effective compared to CAU, as the corresponding ICERs rise well above any acceptable ceiling ratio in The Netherlands (€346,889 and $€ 641,068$, respectively). Comparing KD with VNS, our results suggest that KD dominates VNS (i.e. KD is more effective and less costly than VNS), at least for the first 12 months. At 5 years, KD is still slightly less costly, but also less effective than VNS. There are two main reasons for this shift in outcome: Firstly, the initial (high) costs of the surgical procedure associated with VNS including the device itself are spread over 5 years. Therefore, the expected costs become much lower for each subsequent year. Secondly, there is a delayed response to the VNS treatment. Based on the meta-proportion analysis of the open-label follow-up of the VNS RCTs there is still an increase in the number of responders after 6 months ${ }^{16,17,19}$, whereas the number of responders of the KD ceases to increase. ${ }^{18}$

From an economic perspective and based on these results, one could argue that a child with intractable epilepsy should first be treated with the KD before trying VNS; firstly, during the first 12 months, KD is cheaper and more effective than VNS. Secondly, the effects of the KD are evident after 3 months of treatment and, in case of a responder, achieved with a non-invasive treatment. Finally, in case the patient is a non-responder, VNS remains a treatment option.

Our results are however, sensitive to change, as can been seen in the sensitivity analyses, where we increased the percentage of classical diet users from $15 \%$ to $100 \%$ and simultaneously lowered the KD initiation costs by assuming that no hospitalization is required. This resulted in a higher probability for KD to be cost effective at 12 months and at 5 years. This implicates that in countries where only the classical variant of the KD is offered and initiated without hospitalization, that there is a higher probability that the KD is a cost-effective treatment option in the long-term compared to VNS and CAU (see Figure 8.3B). However in countries where the MCT variant of the diet is offered, there is a higher probability that the VNS is a costeffective treatment option in the long-term compared to KD and CAU (see Figure 8.3D).

Every study has some strengths and weaknesses. One of the strengths of this study is that we constructed a model that enabled us to directly compare the long-term (5 years) costs and effects of three clinically competitive treatment options, despite the lack of comparative studies between all scenarios. Based on such evaluations, decisions are made about the implementation and/or reimbursement of treatments. Furthermore, we validated the structure and the input parameters of the model with a team of experts from different fields. One of the major limitations of the study is that we were unable to predict the outcomes over a longer time horizon. Ideally, an economic model of epilepsy should have a lifetime horizon in order to capture the relevant outcomes in terms of the mortality and morbidity associated with this chronic disease. The long-term prognosis for patients with intractable epilepsy is, 
however, extremely difficult to estimate without a strong body of high quality literature. Therefore, the model was initially estimated with a time horizon of 12 months. We felt, however, sufficiently confident to calculate the cost-effectiveness over a longer period, i.e. 5 years. Based on the open-label extension phases of the RCTs, 12 months of data were available for the KD arm ${ }^{18}$ and 18 months' data for the VNS arm. ${ }^{17}$ In all treatment options, we remained conservative by assuming transitions would not change after the time horizon of the available clinical data. Second limitation is that we used mainly Dutch costs. As costs in other jurisdictions will be different from the Dutch situation, our results could be less generalizable to other health systems. Given our detailed presentation of the model and its input parameters, interested readers can assess the transferability of the results to their specific situation. ${ }^{31}$

Third limitation of the study is the quantity and quality of the synthesized data. We used clinical data of published RCTs and their open-label follow-up periods which are likely to contain considerable uncertainty. Next to this, there are no studies directly comparing KD with VNS, and we found only two published RCTs that compared KD to CAU. ${ }^{10,11}$ Only three RCTs (reporting on unique patients) compared VNS to CAU ${ }^{12-14}$, of which only Klinkenberg et al. (2012) ${ }^{13}$ included exclusively children $<18$ year of age in their analyses. Furthermore, for a number of resource utilization and cost parameters we solely had to rely on expert opinions. Finally, in order to present the costs per QALY we had to allocate absolute utilities to relative health states, as the model is depending on the outcome measures used in clinical trials (i.e. $>50 \%$ seizure reduction). However, a reduction in seizure frequency from two to one seizure per day is probably, in terms of utilities, not the same as a reduction from 50 to 25 seizures per day. Moreover, modeling always involves simplification, and a model should not be faulted because available data do not meet the ideal standards of scientific evidence. ${ }^{32}$

Our results show that the benefits of KD and VNS fail to outweigh the costs of the therapies. However, it would be clinically incorrect to state that these options should be ignored in the treatment of intractable epilepsy. Dugan and Devinsky $(2013)^{33}$ commented on the most recent VNS guidelines, ${ }^{34}$ by saying that in the view of a critical reviewer there is only weak scientific evidence on the effectiveness of VNS. In the view of a clinician, however, VNS has much to offer. Apparently there are, although not reported in the included RCTs, other factors beyond seizure frequency which determine the outcome of these non-AED treatments in intractable epilepsy. For example, positive effects are reported on behavior, mood, alertness, playfulness and interaction both for $\mathrm{KD}^{35-37}$ and VNS. ${ }^{38-41}$ Therefore, it would be wise to include a broader assessment of effectiveness in each clinical trial, preferably in terms of quality of life. Furthermore, clinicians are in desperate need to offer an alternative when antiepileptic drugs are failing in this devastating disorder. The (further) development of these (and other) alternatives should be advocated for and, besides this, the identification of subgroups responding to the different treatments is essential. There 
is a need for more long-term follow-up studies comparing KD with VNS and CAU following the course of intractable epilepsy, including an assessment of quality of life and subgroup analyses to be able to better inform clinicians and policy makers.

\section{Conclusion}

Currently, decisions are taken worldwide, and so it is better to inform these decisions with the best available evidence under conditions of uncertainty, than without any evidence at all. Although our results should be interpreted with great caution, they are potentially meaningful for clinicians and policymakers. From an economic viewpoint, neither VNS nor KD is a cost-effective option compared to CAU at 12 months. In the long-term, the probability of VNS being cost-effective increases to $51 \%$ at 5 years (at a ceiling ratio of $€ 80,000$ per QALY). The probability of KD being cost-effective becomes $63 \%$ at 5 years when all patients are treated with the classical $K D$, without hospitalization during KD initiation.

There is a great need for high quality comparative studies with large patient samples which allow for subgroup analyses, long-term follow-up periods and outcome measures that measures effects beyond seizure frequency (e.g. quality of life). It is important that when new evidence becomes available, reassessment of the costeffectiveness of KD and VNS in children with intractable epilepsy is carried out. The present model could be used for this future evaluation. 


\section{References}

1. Sillanpaa M, Schmidt D. Natural history of treated childhood-onset epilepsy: prospective, long-term population-based study. Brain. 2006;129:617-24.

2. Kossoff EH, Zupec-Kania BA, Amark PE, Ballaban-Gil KR, Bergqvist AGC, Blackford R, Buchhalter JR, Caraballo RH, Cross H, Dahlin MG, Donner EJ, Klepper J, Jehle RS, Kim HD, Christiana Liu YM, Nation J, Nordli DR jr, Pfeifer HH, Rho JM, Stafstrom CE, Thiele EA, Turner Z, Wirrell EC, Wheless JW, Veggiotti $P$, Vining EPG. Optimal clinical management of children receiving the ketogenic diet: Recommendations of the international ketogenic diet study group. Epilepsia. 2009;50:304-17.

3. Freeman J, Veggiotti P, Lanzi G, Tagliabue A, Perucca E. The ketogenic diet: from molecular mechanisms to clinical effects. Epilepsy Res. 2006;68:145-80.

4. Groves DA, Brown VJ. Vagal nerve stimulation: a review of its applications and potential mechanisms that mediate its clinical effects. Neurosci Biobehav Rev. 2005;29:493-500.

5. Levy RG, Cooper PN, Giri P. Ketogenic diet and other dietary treatments for epilepsy. Cochrane Database Syst Rev. 2012;3:CD001903.

6. Privitera MD, Welty TE, Ficker DM, Welge J. Vagus nerve stimulation for partial seizures. Cochrane Database Syst Rev. 2002 (1):CD002896.

7. Siebert U, Alagoz O, Bayoumi AM, Jahn B, Owens DK, Cohen DJ, et al. State-transition modeling: a report of the ISPOR-SMDM Modeling Good Research Practices Task Force--3. Value Health. 2012;15: 812-20.

8. Eddy DM, Hollingworth W, Caro JJ, Tsevat J, McDonald KM, Wong JB, et al. Model transparency and validation: a report of the ISPOR-SMDM Modeling Good Research Practices Task Force--7. Value Health. 2012;15:843-50.

9. Evans C. The use of consensus methods and expert panels in pharmacoeconomic studies. Practical applications and methodological shortcomings. Pharmacoeconomics. 1997;12:121-9.

10. Neal EG, Chaffe H, Schwartz RH, Lawson MS, Edwards N, Fitzsimmons G, et al. The ketogenic diet for the treatment of childhood epilepsy: a randomised controlled trial. Lancet Neurol. 2008;7:500-6.

11. Sharma S, Sankhyan N, Gulati S, Agarwala A. Use of the modified Atkins diet for treatment of refractory childhood epilepsy: a randomized controlled trial. Epilepsia. 2013;54:481-6.

12. Handforth A, DeGiorgio CM, Schachter SC, Uthman BM, Naritoku DK, Tecoma ES, et al. Vagus nerve stimulation therapy for partial-onset seizures: a randomized active-control trial. Neurology. 1998;51: 48-55.

13. Klinkenberg S, Aalbers MW, Vles JS, Cornips EM, Rijkers K, Leenen L, et al. Vagus nerve stimulation in children with intractable epilepsy: a randomized controlled trial. Dev Med Child Neurol. 2012;54: 855-61.

14. Group TVNSS. A randomized controlled trial of chronic vagus nerve stimulation for treatment of medically intractable seizures. The Vagus Nerve Stimulation Study Group. Neurology. 1995;45:224-30.

15. Ben-Menachem E, Manon-Espaillat R, Ristanovic R, Wilder BJ, Stefan H, Mirza W, et al. Vagus nerve stimulation for treatment of partial seizures: 1. A controlled study of effect on seizures. First International Vagus Nerve Stimulation Study Group. Epilepsia. 1994;35:616-26.

16. DeGiorgio CM, Schachter SC, Handforth A, Salinsky M, Thompson J, Uthman B, et al. Prospective longterm study of vagus nerve stimulation for the treatment of refractory seizures. Epilepsia. 2000;41:1195-200.

17. George R, Salinsky M, Kuzniecky R, Rosenfeld W, Bergen D, Tarver WB, et al. Vagus nerve stimulation for treatment of partial seizures: 3. Long-term follow-up on first 67 patients exiting a controlled study. First International Vagus Nerve Stimulation Study Group. Epilepsia. 1994;35:637-43.

18. Neal EG, Chaffe H, Schwartz RH, Lawson MS, Edwards N, Fitzsimmons G, et al. A randomized trial of classical and medium-chain triglyceride ketogenic diets in the treatment of childhood epilepsy. Epilepsia. 2009;50:1109-17.

19. Salinsky MC, Uthman BM, Ristanovic RK, Wernicke JF, Tarver WB. Vagus nerve stimulation for the treatment of medically intractable seizures. Results of a 1-year open-extension trial. Vagus Nerve Stimulation Study Group. Arch Neurol. 1996;53:1176-80. 
20. Age related mortality [Internet]. 2012 [cited 02-02-2014]. Available from:

http://statline.cbs.nl/StatWeb/publication/?DM=SLNL\&PA=37530ned\&D1=1\&D2=101-

120\&D3=0\&D4=I\&HDR=T,G1\&STB=G2,G3\&VW=T.

21. Shorvon S, Tomson T. Sudden unexpected death in epilepsy. Lancet. 2011;378:2028-38.

22. Messori A, Trippoli S, Becagli P, Cincotta M, Labbate MG, Zaccara G. Adjunctive lamotrigine therapy in patients with refractory seizures: a lifetime cost-utility analysis. Eur J Clin Pharmacol. 1998;53:421-7.

23. Salomon JA, Vos T, Hogan DR, Gagnon M, Naghavi M, Mokdad A, et al. Common values in assessing health outcomes from disease and injury: disability weights measurement study for the Global Burden of Disease Study 2010. Lancet. 2012;380:2129-43.

24. Suh GH, Lee SK. Economic Evaluation of Add-on Levetiracetam for the Treatment of Refractory Partial Epilepsy in Korea. Psychiatry investigation. 2009;6:185-93.

25. Verdian L, Yi Y, Oyee J, Heyes A, Tolley K, editors. Eliciting preferences for health states associated with lennox-gastaut syndrome (LGS). Poster 1352 presented at the 62nd meeting of the American Epilepsy Society; 2008.

26. Board. $\mathrm{HCl}$. Guidelines for pharmaco-economic research (in Dutch). Diemen: Health Care Insurance Board; 2006.

27. Guerrini R, Battini R, Ferrari AR, Veggiotti P, Besana D, Gobbi G, et al. The costs of childhood epilepsy in Italy: comparative findings from three health care settings. Epilepsia. 2001;42:641-6.

28. Hakkaart - van Roijen L, Tan SS, Bouwmans CAM. Handleiding voor kostenonderzoek. Amstelveen: CVZ; 2010.

29. Drummond MF, Sculpher MJ, Torrance GW, O'Brien BJ, Stoddart GL. Methods for the economic evaluation of health care programs. Oxford: Oxford University Press; 2005.

30. Council for Public Health and Health Care. Sensible and sustainable care (in Dutch). Zoetermeer: Council for Public Health and Health Care; 2006.

31. Welte $\mathrm{R}$, Feenstra $\mathrm{T}$, Jager $\mathrm{H}$, Leidi $\mathrm{R}, \mathrm{A}$ decision chart for assessing and improving the transferability of economic evaluation results between countries. Pharmaeconomics. 2004;22);857-76.

32. Weinstein MC, O'Brien B, Hornberger J, Jackson J, Johannesson M, McCabe C, et al. Principles of good practice for decision analytic modeling in health-care evaluation: report of the ISPOR Task Force on Good Research Practices--Modeling Studies. Value Health. 2003;6:9-17.

33. Dugan P, Devinsky O. Epilepsy: Guidelines on vagus nerve stimulation for epilepsy. Nat Rev Neurol. 2013;9:611-2.

34. Morris GL, 3rd, Gloss D, Buchhalter J, Mack KJ, Nickels K, Harden C. Evidence-based guideline update: vagus nerve stimulation for the treatment of epilepsy: report of the guideline development subcommittee of the american academy of neurology. Epilepsy currents / American Epilepsy Society. 2013;13:297-303.

35. Farasat S, Kossoff EH, Pillas DJ, Rubenstein JE, Vining EP, Freeman JM. The importance of parental expectations of cognitive improvement for their children with epilepsy prior to starting the ketogenic diet. Epilepsy Behav. 2006;8:406-10.

36. Hallbook T, Lundgren J, Rosen I. Ketogenic diet improves sleep quality in children with therapyresistant epilepsy. Epilepsia. 2007;48:59-65.

37. Pulsifer MB, Gordon JM, Brandt J, Vining EP, Freeman JM. Effects of ketogenic diet on development and behavior: preliminary report of a prospective study. Dev Med Child Neurol. 2001;43:301-6.

38. Hallbook T, Lundgren J, Stjernqvist K, Blennow G, Stromblad LG, Rosen I. Vagus nerve stimulation in 15 children with therapy resistant epilepsy; its impact on cognition, quality of life, behaviour and mood. Seizure. 2005;14:504-13.

39. Kang HC, Hwang YS, Kim DS, Kim HD. Vagus nerve stimulation in pediatric intractable epilepsy: a Korean bicentric study. Acta neurochirurgica Supplement. 2006;99:93-6.

40. Majoie HJ, Berfelo MW, Aldenkamp AP, Renier WO, Kessels AG. Vagus nerve stimulation in patients with catastrophic childhood epilepsy, a 2-year follow-up study. Seizure. 2005;14:10-8.

41. Shahwan A, Bailey C, Maxiner W, Harvey AS. Vagus nerve stimulation for refractory epilepsy in children: More to VNS than seizure frequency reduction. Epilepsia. 2009;50:1220-8. 
Part 11

Discussion and summary 


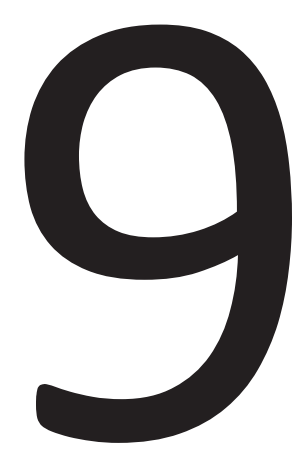

General discussion 
Chapter 9 


\section{General discussion}

The general aim of this thesis, as introduced in Chapter 1 , is two-fold: I) to address a number of methodological issues concerning outcome assessment and, II) to estimate the costs, benefits and cost-effectiveness of the ketogenic diet (KD) and vagus nerve stimulator (VNS) as treatment options for children with intractable epilepsy. In order to provide insights into the general aim, this thesis is structured around three research questions. This chapter opens with a presentation of the main conclusions of the research questions. Then some discussion themes regarding Health Technology Assessment (HTA) research in epilepsy are highlighted, followed finally by some recommendations.

\section{Research question 1}

\section{What are the preferences for outcome measures and diagnostics in epilepsy?}

One of the major challenges in HTA research is to obtain a suitable and valid measure for the benefit of (medical) interventions to be investigated in economic evaluations. When health care resources are limited, efforts are made to prioritize the acquisition and the use of (new) interventions. It is, therefore, important that outcomes in different fields are comparable. By estimating health benefits in terms of Quality Adjusted Life Years (QALYs), it is theoretically possible to compare directly the costs of achieving such health benefits. Utilities are essential in order to calculate QALYs; however, utility estimates are seldom available. Especially in the case of model-based, cost-utility analyses, where researchers are dependent on the published literature, suitable utilities for health states are hard to find. Utilities can be indirectly measured with generic preference-based quality of life (QoL) instruments (e.g. EQ-5D) or directly using specific eliciting methods (e.g. Time Trade-off (TTO), see also chapters 1 and 2). Utility values necessary for HTA research are (usually) not measured in clinical studies regarding epilepsy, which mostly focus on clinically relevant outcome measures such as seizure reduction. A uniform understanding of the implication of a seizure reduction on a patient's health state utility is lacking. Many studies report an association between seizure frequency with poorer $\mathrm{QoL}^{1,2}$; others have found a significant association only when patients became seizure-free ${ }^{3}$ or have not found such an association at all. ${ }^{4,5}$ Furthermore, some others argue that in case of intractable epilepsy, seizure frequency may be less important than patient-perceived seizure severity or side-effects due to antiepileptic drugs in predicting QoL., ${ }^{6,7}$

To obtain more insight into factors affecting QoL, we researched (chapter 2) preferences for three clinical outcome measures: seizure frequency, seizure severity, and side-effects, and we estimated utility values for 78 health states based on a combination of these clinical outcome measures. With this utility function, outcomes 
of studies reporting on clinical outcome measure regarding seizure frequency, seizure severity and side-effects can be transformed into utility values suitable for economic evaluations. Until now, this is the best 'bridge' between clinical epilepsy outcome measures and HTA relevant outcome measures. The study described in chapter 2 showed that seizure frequency, seizure severity and side-effects have a significant impact on QoL. Furthermore, it is concluded that seizure severity is the largest predictor of QoL. The study was conducted from a societal perspective. Hence, the general population was provided with a web-based questionnaire and asked to express their preferences for epilepsy health states.

One may consider it a logical choice to ask patients in specific health states to value their own health. While this indeed has been advocated ${ }^{8}$, it is relatively unusual to do so, for several reasons. Firstly, patients may adjust to their poor health and hence, they value their health state better than non-patients ${ }^{9-11}$ (i.e. adaptation); however, other observations also exist. ${ }^{12,13}$ Second and third arguments used to obtain preferences from the general public are: II) the societal perspective ${ }^{14}$, and III) the insurance principle. ${ }^{15}$ These arguments are considered to be sufficiently convincing and supportive of the view that the value of a health state should solely be determined by general public preferences. Others argue that completely disregarding patient preferences cannot be based on the arguments put forward in the literature and show that using societal preferences may result in either higher or lower estimates of cost-effectiveness when compared to using values obtained in patients. When only one source of information is prescribed in health care decision-making, there is a risk of losing information that is potentially relevant to the decision-making context, and therefore, such a choice requires justification.

In chapter 2, preferences for epilepsy health states are measured with the Time Trade Off (TTO) method. Another method of eliciting preferences is by means of a Discrete Choice Experiment (DCE). ${ }^{16}$ DCEs have been used to examine, e.g. the choice of which car to buy ${ }^{17}$, where to go to college ${ }^{18}$, which mode of transport (car, bus, train) to take to work $^{19}$, what telephone service a customer decides to purchase ${ }^{20}$, among numerous other applications. In recent years, there has been a growing interest in the application of DCEs within health economics shown by the enormous increase in literature applying this technique. ${ }^{21}$ In the health care sector, DCEs provide rich data sources for economic evaluation and decision-making, allowing investigation of many types of questions, some of which would otherwise be intractable analytically. ${ }^{22}$

DCEs are used to elicit patient preferences for treatments in general, and have been used previously to estimate preferences of patients with epilepsy regarding sideeffects of antiepileptic drugs (AEDs). ${ }^{23}$ Knowledge on patients' preferences can be used for the creation of new interventions or treatments, and can be informative for clinicians in the decision-making process. It is, however, unclear whether patients' preferences regarding treatments and their potential adverse effects are always considered in the clinical decision-making process, which is, in the field of epilepsy, primarily concerned with balancing seizure control and adverse effects. It has been 
advocated that the management of a disease like epilepsy should incorporate a shared decision-making process between doctor and patient. ${ }^{24,25}$

Chapter 3 shows the possibility of retrieving preferences for diagnostic procedures in an epilepsy patient population by means of a DCE. This study was designed to identify to what extent important attributes of diagnostic procedures in epilepsy affected preferences for a procedure, and to determine the relative importance of these attributes. Participants were asked to state their preferred choice between two (or more) scenarios based on these attributes.

When looking at the relative importance weights, two attributes stand out: "chance of additional examinations" and "freedom of movement". With regard to the latter, we anticipated the fact that patients would prefer recordings in which minor movements are possible. Beforehand, however, it was not thought that it would be the most influential factor in determining patients' preferences.

Not all results presented in this chapter were straightforward or in line with the expectations a-priori. Patients' preferences may sometimes be included in decisionmaking, but this is probably in the form of the physician's perceptions of patients' preferences, which may be biased. DCEs seemed to be a useful method of eliciting preferences because they are able to quantify the individual impact of each different attribute of the intervention, in this case diagnostic procedures, on decision-making.

\section{Research question 2}

\section{What is the economic burden of side-effects due to AEDs in epilepsy?}

The annual costs of epilepsy in Europe are $€ 15.5$ billion. ${ }^{26}$ In the Netherlands, direct medical costs in 2011 were $€ 248$ million, which is $0.3 \%$ of the health care budget. ${ }^{27}$ The main cost driver is health care professional visits and hospitalizations including diagnostic procedures (e.g. MRI, EEG), accounting for almost half of the total direct medical costs ( $€ 122$ million). Institutionalized patients are the second main cost driver, accounting for €73.6 million and the third main cost driver is the cost of medication (€36.5 million). ${ }^{27}$ Although epilepsy is a disease with a high medication usage, the costs of medications account for less than $15 \%$ of the total medical costs. However, when patients need to change their medications or experience (any) sideeffects they will visit their attending physician or the hospital, resulting in higher actual medication costs.

The majority of medications used in epilepsy are AEDs. There are (theoretically) many AEDs available; currently, approximately 25 different AEDs, which can be very successful in controlling seizures in about $70 \%$ of the patients; side-effects are, however, common. In order accurately to reflect the total management costs, pharmaco-economic analyses should include the evaluation of costs related to negative outcomes of pharmacotherapy, such as adverse drug reactions. The costs of serious adverse cutaneous or hypersensitivity reactions due to AEDs have been 
estimated to be more than CAN\$3,000 per affected patient. ${ }^{28}$ Serious adverse events are often treated with very expensive hospitalizations and medications. Such events are, however, rare, occurring in less than $1 \%$ of patients treated. ${ }^{29,30}$

When estimating common side-effects due to AEDs, about $60 \%$ of the patients report side-effects in at least three of the following domains: general central nervous system, motor problems, gastrointestinal complaints, cognition, visual, mood and behavior, cosmetic or sleeping problems. ${ }^{31}$ Subjectively reported common side-effects are not likely to induce such very expensive hospitalizations; however, costs of (over the counter) medication and visits to the general practitioner, specialist or paramedic can aggregate to high costs, especially when such side-effects are chronic. In chapter 4 we explored the societal costs of common, subjectively reported, side-effects and concluded that the overall societal costs due to side-effects in patients from the tertiary epilepsy center amounted to more than $€ 20,000$ per patient per year, of which almost a quarter was related to direct medical costs $(€ 4,475)$. The advice is, therefore, that costs of side-effects should be considered in economic evaluations of pharmacotherapy. The above estimation was solely related to side-effects due to AEDs; other treatments, however, such as the ketogenic diet or vagus nerve stimulator also induce side-effects. An equivalent comparison between therapies, should take the costs due to side-effects of all therapies into account.

\section{Research question 3}

\section{What are cost-effective options in the treatment of intractable epilepsy?}

The sustainability of health care expenditure is under pressure. In 2012, €83 billion was spent on health care in the Netherlands, almost $€ 5,000$ per person. ${ }^{32}$ Our health care expenditure is similar to that of Germany and France, though overall higher than other European countries. If health care expenditure continues to rise as it has done during the past decade, health care costs will reach $€ 8,500$ per person in 2030 . $^{32}$

In the Netherlands, solidarity is a great good; young or old, sick or healthy, if someone needs medical support this should be accessible for everyone. But with continuously rising costs, solidarity will come under pressure. Most people consider good health care to be one of the most important provisions which requires substantial funding. Younger generations with an average wage with no or low usage of health care wonder if there is a proper balance between paying for health insurance now and the consumption of health care later. A lack of confidence in and responsibility towards the solidarity principle of our health care system has occurred due to ever-rising health care expenditures, insurance payments and increased deductibles. To interrupt this downward spiral, we need to make choices in health care (see also chapter 1). Investigating which interventions are the most cost-effective for certain health conditions is part of the information needed by policy makers in order to allow them 
to prioritize interventions in a scarce environment. As introduced in chapter 1 , there are several steps involved in determining whether or not a (medical) intervention is more cost-effective than usual care in economic evaluations. For a meaningful comparison, it is necessary to examine the additional costs that one medical intervention imposes over another, compared with the additional effects or utilities it delivers.

In chapter 5, a systematic review is presented considering full economic evaluations of therapies for intractable epilepsy. Systematic reviews aim to identify relevant studies in a particular topic area, appraise their quality and summarize their results using a scientific methodology. ${ }^{33}$ In total, 12 articles were included, all presenting full economic evaluations of add-on AEDs. The outcome measures used in the included articles differed (e.g. cost per seizure-free day, per responder, per seizure-free patient, per QALY), making comparison difficult. The large differences in costs found between studies is largely due to the perspective of the economic evaluation and thus the cost factors taken into account. For example: the results ranged from $£ 761$ to $£ 154,831$ per QALY, for respectively, zonisamide followed by lamotrigine compared with levetiracetam followed by lamotrigine and, topiramate compared with lamotrigine. Furthermore, the costs per responder ranged from €954 per patient with $50 \%$ seizure reduction to $f 5,301$ per seizure-free patient. A certain amount of heterogeneity between the economic evaluations is unavoidable, because of the multiple choices available when defining parameters of economic evaluations (e.g. designs, outcome measures, patient samples, etcetera, see also chapter 1 ). On the other hand, other areas of heterogeneity can be avoided, simply by following standard economic research methods. ${ }^{34-37}$

Besides being unable to give a conclusive answer to research question 3 after reviewing multiple AED studies in patients with intractable epilepsy, no studies were identified evaluating the KD or VNS.

In order to answer research question 3 as far as the KD and VNS are concerned, two studies were conducted which differed methodologically. First of all, a trial-based economic evaluation was conducted (the KOEK-study) in which the KD was compared with care as usual (CAU) in a RCT (chapter 7). Calculating the cost per QALY at 4months resulted in an unfavorable outcome for KD compared to CAU (i.e. KD was more costly and generated an equal amount of QALYs compared to CAU, see chapter 7 ). However, comparing the number of responders (i.e. $\geq 50 \%$ seizure reduction) showed a significant difference in favor of KD.

Secondly, a model-based economic evaluation with QALYs as primary outcome measure was conducted, in which the KD and VNS were compared with CAU (chapter 8). Here, the estimated QALYs were based on published utilities, (partly) suitable for our clinical health states. At one year follow-up, neither KD nor VNS seemed to be cost-effective at any given ceiling ratio. However, both therapies appeared to be more effective than CAU regarding number of responders. In the long-term (i.e. 5-year), 
VNS and KD have a probability of being cost effective of $31 \%$ and $7 \%$ respectively at a ceiling ratio of $€ 50,000$.

Following the Dutch guidelines on decision-making related to health care, an ICER between $€ 20,000$ and $€ 80,000$ can be cost-effective. The exact height of the ceiling ratio depends on the burden of disease. The ceiling ratio is higher for a disease with a high burden than for a disease with a low burden. In the case of intractable epilepsy, which has a burden of disease of 0.657 , interventions will be cost-effective when the cost per QALY lies below a ceiling ratio of $€ 50,000$. $^{38,39}$ Neither the research presented in chapter 7 nor in chapter 8 can comply with these guidelines. One might wonder if the economic evaluation methods and frameworks used are out of touch with such obvious perceptions of clinical necessity, or are the ketogenic diet and vagus nerve stimulator simply too expensive?

The conclusion that a clinician would reach, and that a policy-maker should make, both based on our results, are (possibly) completely distinct from each other. Obviously, further investigation is necessary to explain these differences. A discussion follows below on several themes regarding HTA in epilepsy.

\section{HTA in epilepsy: Bringing two worlds closer together}

For both clinicians and policy-makers, evidence-based research is a great good. The way they regard such evidence may, however, in some cases be completely different. Studies investigating the uptake of HTA-studies in clinical practice found that this uptake is generally limited. ${ }^{40-43}$ Most of these studies have also revealed that with respect to evidence-based research, clinical aspects have a greater influence on clinical decision-makers. HTA research, including economic evaluations, are often considered to be of secondary importance. Furthermore, the lack of economic evaluations in the field of epilepsy can be attributed to both limited funding for such studies $^{36}$, and the fact that evaluation methods are insufficiently developed to deal with the challenges posed in epilepsy research. In the present thesis, we conducted HTA research in the field of epilepsy and also, in all modesty, tried to bridge the gap between clinical epilepsy and HTA research. A discussion of some challenges we faced will follow below.

\section{Benefits for HTA and epilepsy research}

Our health care systems are continuously being offered new drugs, devices and procedures, most of which produce health benefits. These benefits, however, often entail considerable additional costs for society; and as health care resources are limited, efforts are made to prioritize the acquisition and the use of technologies. In order to be able to prioritize health care, it is important that outcomes of different health conditions can be compared. Since it is difficult to compare very different health outcomes (e.g. epileptic seizures prevented vs headaches prevented), HTA researchers use the measure, QALY. A QALY incorporates effects in terms of both QoL 
(utilities) and survival (life-years gained). By estimating all health benefits in terms of QALYs it is theoretically possible to compare the costs of achieving different health outcomes directly.

Despite a long-recognized need for QALYs in the broader priority-setting field and the growing evidence that psychosocial factors and poor mental health have the greatest impact on QoL ${ }^{5,44,45}$, the clinical community has continued to focus primarily on seizures and their treatment.

In Chapter 7 we found a significant difference in the number responders in the ketogenic diet group compared with the care as usual group. We did not, however, find any difference in QALYs between the two groups. Finding discrepancies between the main clinical epilepsy outcome measure (i.e. $\geq 50 \%$ seizure reduction) and the main HTA outcome measure (i.e. QALYs) is not a motivating basis in the attempt to bring the two worlds closer together. So how is it possible that we found such a discrepancy between the BTA and the clinical measure? Is being a responder not an indicator for increased QoL in patients with intractable epilepsy? Or is the measurement instrument we used insensitive to clinical improvements in these patients?

On the one hand, the critical attitude of clinicians towards the QALY is not totally groundless. Besides the discrepancy between the clinical and HTA outcome measures, there are more caveats to the measurement of the QALY in epilepsy. Firstly, a common method for measuring a person's health state is through standardized generic preference-based QoL questionnaires, on which participants can indicate how they score in different predefined health domains. The article by Leung et al. (2013) points to a general problem with these instruments. In their comparison of the EQ-5D and SF-6D, the authors find dissimilar distributions of utilities, different ceiling effects, different mean scores, and a low correlation between the two sets of results. ${ }^{46}$ This is a disturbing finding, since the 2 instruments purport to measure the same construct, namely, utility (the strength of preference for a certain health state). One might expect the differences in results found between the instruments to lead to a critical review of both instruments, or perhaps even to the conclusion that one or both instruments are defective. To the contrary, however, the EQ-5D and the SF-6D have coexisted with relatively little criticism for 15 years and both have been widely used for the evaluation of health services and products. ${ }^{47}$

These results highlight an unsatisfactory state of measurement theory and practice with respect to the generic preference-based utility instruments which measure QoL. Economists who have created the instruments have focused almost exclusively upon the derivation of the 'utility-tariff' ${ }^{48}$ from the general population, which is important for the second part of the instrument. Despite the existence of well-established psychometric methods, they have largely ignored the reliability and validity of the first part of the instrument, that is, the questionnaire used to obtain the health state description from a patient. As a consequence, the precision of clinical measurement in evaluation studies may well be offset by the unreliability of the measurement 
instrument, and acceptance or rejection of a therapy by a national health service may be dependent upon the QoL instrument chosen.

Secondly, the sole function of a QALY is to enable decision-makers to compare the cost of very different health conditions. To achieve this, all existing QALYs must have been valued equally; however, the value accorded to them varies with circumstance. For example, there is evidence that the value given to a health state differs per method used (e.g. TTO, SG, or VAS) ${ }^{49-51}$, (methodological) context (e.g. lead-time and lag-time TTO $)^{52}$, and is, as described earlier, dependent on whose values are taken into account. ${ }^{9,10}$

Besides this critical appraisal towards the use of QALYs in epilepsy research, previous research has shown that measuring only clinical outcome measures is not a suitable solution. Research over the past 20 years has demonstrated that changes in seizure rates among patients with intractable epilepsy are only modestly correlated with QoL. ${ }^{53-55}$ As Perucca and colleagues suggest, "the lack of investigations towards other clinical factors can be ascribed to historical belief that seizure frequency is the major determinant of QoL". ${ }^{7}$ There are possible other clinical factors, or even non-health factors that are strongly related to QoL. Patients with epilepsy have a significantly higher prevalence of psychological and psychiatric comorbidity compared with the population without epilepsy. ${ }^{56}$ For example, the lifetime prevalence of depression in patients with epilepsy ranges from $29 \%-50 \%{ }^{57}$; the range in the general population is $16-22 \%{ }^{58,59}$ Furthermore, social and interpersonal stresses that a patient with epilepsy encounters through stigma, social isolation and unemployment also has an impact on their QoL. ${ }^{60,61}$ Specific risk factors associated with QoL in children consist of a variety of clinical and family factors. ${ }^{62}$ As there is growing evidence that psychosocial factors and poor mental health have the greatest impact on QoL in patients with epilepsy $^{5,63,64}$, Elliot and Richardson ${ }^{65}$ opt for a shift from the biological-biomedical model to a bio-psychosocial model in order to understand QoL. Furthermore, patients with intractable epilepsy commonly suffer from other co-morbidities (e.g. mental retardation, depression) which could have a greater negative impact on QoL than epilepsy alone. The impact of such co-morbidity can substantially impair QoL, making $a \geq 50 \%$ seizure reduction gain (or not) more or less negligible.

Despite this accumulating evidence, experts acknowledge that neurologists tend to focus on the control of seizures and lack further interest in psychosocial aspects of epilepsy. ${ }^{66}$ Such psychosocial aspects have a strong association with QoL; therefore, the bio-psychosocial model may be the missing link between clinical outcome measures and QALYs. In order to calculate QALYs suitable for economic evaluations, utilities based on generic QoL measures have to be elicited. Perhaps even more importantly, effort should be put into finding (or developing) a valid and reliable preference-based generic QoL instrument to use in epilepsy research. There are other preference-based generic QoL instruments under development which are potentially suitable for epilepsy research, such as the AQoL-8D or the ICECAP. ${ }^{47,67}$ The 
psychometric properties of the QoL instruments within the field of epilepsy do, however, need to be researched before implementation.

Despite ongoing debates in the literature, there appears to be a relatively strong practical consensus regarding the use of QALYs. This practical consensus is represented by existing guidelines, such as the 'Guide to the methods of technology assessment' of the National Institute for Clinical Excellence (NICE), which attempts to guard quality and uniformity in terms of methodology of the measurement of effects for cost-utility analysis. In accordance with the NICE guidelines, the Dutch Board of Health Insurance (CVZ) also mentions the EQ-5D as the preferred outcome measure ${ }^{68}$, the use of TTO as preferred utility elicitation method, values from the general population and (if necessary) the application of mapping techniques.

If HTA researchers wish clinicians to use QALYs, and decision-makers to use the results of cost-utility studies, it is of utmost importance that the QALY as an outcome measure is methodologically valid and unbiased, since decisions based on the results of cost-utility studies may affect the wellbeing and welfare of many individuals.

\section{Evidence-based decision-making}

In an era of evidence-based decision-making, the importance of using results from scientific research in practice is not in doubt. The scientific community must, therefore, invest in translating new insights into useful guidelines for practice and to deliver answers to practice-related questions. In the case of HTA research in epilepsy, this means that research must be translated into clinical and policy decisions.

The translational gap between research and practice has often been discussed as a one-way flow: getting practitioners to recognize and utilize the research that is being conducted. While the other way around is possibly equally important, namely: integrating practice-based evidence and context into the research conducted.

Therefore, long-term costs and outcomes should be considered more often in (economic) evaluation studies to provide insight into all relevant costs and outcomes of interest to decision-makers. Given that long-term trials, or performing an intervention trial and a subsequent model-based study to extrapolate the trial findings, can be both time-consuming and costly, one might wonder whether decision-makers should be less critical about so-called 'low quality data'. Most of the scientific evidence base is largely inadequate for clinical practice, as these data are derived from regulatory trials which are too short for a chronic condition and use outcomes of dubious relevance. ${ }^{69,70}$ These studies are double-blind, randomized, controlled trails and rated as high quality evidence, while longer term unblinded pragmatic studies, that might actually inform a clinical decision, are considered to be low quality.

The RCTs will have a high internal validity which will provide answers to the question whether the difference between groups is due to the intervention rather than to other external influences (i.e. confounders). To keep the study free of confounders, and thus increase the internal validity, the research population must meet the 
predefined inclusion and exclusion criteria and the setting and procedures must serve a detailed protocol. This type of research has an explanatory approach, aimed at understanding. Their effects are assessed by biologically meaningful criteria, and they are applied to a class of patients which is as likely as possible to reveal any difference that may exist. The downside of such a controlled setting is that there is a chance of completely losing any external validity and hence the usefulness in practice. A pragmatic approach would be not to aim at understanding, but at reaching a decision, which seeks to answer the question: which treatment should we prefer? The criteria by which the effects are assessed take into account the interests of the patients and the costs in the widest sense. The class of patients is the one to which the results of the trial are to be extrapolated in the real world. ${ }^{71}$

A recent example of a pragmatic study is the research conducted by Orosz and colleagues. ${ }^{72}$ Their article presents the results of a long-term (i.e. 24 months) study evaluating the effects of the VNS in 347 patients. This study was conducted as a retrospective multicenter study and presented responder rates similar to rates from randomized controlled trials. ${ }^{73,74}$ Although it is very informative, it would be considered as a low quality study, due to its design. The results mimic clinical practice, have a high external validity, can, therefore, be generalized and are highly relevant for evidence-based practice.

In recent years, there is more appreciation for the use of prospective, real-world data. Methodological options and alternatives for gathering data with high external validity are available. One of the options is a multi-center approach in which some of the participating centers apply the intervention while other centers deliver care as usual. In time, patients who are treated with the intervention are compared with patients who are treated with care as usual. During the comparison, patients are matched according to certain variables, such as sex, age and the severity of the disease, and possibly compared with a third group of patients on a waiting list. Another methodological variant leading to real-world data is the pragmatic RCT. In a pragmatic $\mathrm{RCT}$, the intervention is embedded in daily practice, the intervention group is a sample of the targeting population, the measurement of effects are relevant for practice and the perspective of the patient is highly important. Making use of patient registries is another option to collect real-world data prospectively. A patient registry is a prospective observational study on subjects, with certain shared characteristics (e.g. epilepsy), that collects ongoing and supporting data over time on well-defined outcomes of interest for analysis and reporting. ${ }^{75}$ Observational studies are ideally designed for gathering data on the natural cause of disease development. In a study investigating the effect of taking observational data into account for meta-analyses, it was found that including observational studies in meta-analyses may improve the inference based on only randomized trials and furthermore, that observational data generally produce estimates of effect similar to those from meta-analyses based on RCTs. $^{70}$ 
HTA research is increasingly in need of real-world data in order to address aspects of clinical effectiveness and economic evaluation in a policy-relevant manner. With the use of data from registries instead of RCTs, external validity would be traded for internal validity. Patient registries can be created for many purposes: to describe the natural history of disease, to determine clinical effectiveness or cost-effectiveness (including QoL) of health care products and services, to measure or monitor safety and harm, and/or to measure quality of care. Nevertheless, the use of registry data for HTA purposes has been limited and encounters problems of data quality, as well as procedural barriers.

In order to overcome such limitations, it is of utmost importance that a patient registry is designed with respect to its major purpose, with the understanding that different levels of rigor may be required for registries designed to address focused analytical questions to support decision-making, in contrast to those intended primarily for descriptive purposes. There are five key points to consider in designing a registry in order to answer a certain research question: I) choosing a study design, II) translating questions of (clinical) interest into measurable exposures and outcomes, III) choosing patients for study and decide on the need for a comparison group, IV) determining where data can be found, and V) deciding how long and how many patients need to be studied. ${ }^{75}$

A registry in which broad economic, suitable QoL and relevant clinical data of epilepsy patients are systematically gathered can be a real stepping stone for HTA research in epilepsy.

\section{Support decision-making for clinic and policy}

The systematic use of evidence to inform decisions on health care in the Netherlands and other countries is currently hampered by a lack of formal structures to support decision-making. ${ }^{76}$ To encourage more use of economic evaluation evidence and thereby efficient allocation of public resources, clearer decision-making structures need to be developed. The National Institute for Health and Care Excellence (NICE) in London, is one of the first formal organizational structures for systematic and transparent consideration of evidence in decision-making. ${ }^{77}$ Throughout the development of the guidance, views of different stakeholders are considered, including health professionals and patients and caregivers. ${ }^{78}$

The NICE guideline, 'The epilepsies', takes the economic evidence of published economic evaluations on epilepsy treatments into account. ${ }^{79}$ During the construction of 'The epilepsies', health economists allocated an overall score to the existing evidence based on the study limitations and on its applicability for the specific guideline. Full economic evaluations (cost-effectiveness, cost-utility, cost-benefit and cost-consequence analyses) and comparative costing studies that address the review question in the relevant population are considered potentially applicable as economic evidence. The same population and intervention criteria are applied as in the clinical review. The primary criteria applied for an intervention to be considered cost- 
effective are either: I) the intervention dominated other relevant strategies (that is, it is both less costly in terms of resource use and more clinically effective compared with all relevant alternative strategies), or II) the intervention costs less than $£ 20,000$ per QALY gained compared with the next best strategy. ${ }^{79}$

In the Netherlands, the thorough epilepsy guidelines include a number of recommendations about specific clinical problems. The guidelines are based, when possible, on scientific research supplemented by the expertise of health care professionals and patients. The economic evidence has, however, not (yet) been taken into account. The Dutch guidelines acknowledge the importance of taking economic evidence into account. However, they refer to the gaps in knowledge relating to the economic data and question the quality of the small number of economic evaluations. The developers of the Dutch guidelines are not alone in this statement; the NICE guidelines on epilepsy have come under fire from several experts who say that they do not reflect clinical experience and focus too much on drug cost-effectiveness. ${ }^{69}$

In order to increase the quality of economic evaluations, HTA-researchers should comply with methodological standards described in good practice guidelines ${ }^{34-37}$ when planning and designing new economic evaluation studies. The reporting of studies could be improved by using quality assessment checklists, which identify methodological aspects that are essential in economic evaluation (e.g. ${ }^{36,80-82}$ ). Longterm costs and outcomes should be considered more often in economic evaluation studies to provide insights into all relevant costs and outcomes of interest to decisionmakers.

Although the criticism on the NICE-guidelines should not be ignored, the development of a Dutch clinical epilepsy guideline incorporating HTA aspects would be a step forward in bringing the two worlds closer together. Such a guideline, however, should not override the responsibility of health care professionals and others to make decisions appropriate to the circumstances of each patient. These decisions should be based on shared decision-making: in consultation with, and with the agreement of, the patient and/or their guardian or caregiver. In order to further inform guideline developers, health care professionals and others should record their reasons for not following clinical guideline recommendations.

\section{Final recommendations}

Several recommendations for further research emerge from this thesis. The first is to obtain greater insight into the measurement of QoL in (intractable) epilepsy. More research is required in order to investigate the reasons behind our failure to measure improvements in QALYs with generic QoL instruments in patients who were labeled as responders in chapter 7. Obviously, for an economic evaluation to be a legitimate policy tool, its essential elements need to be measured in a methodologically sound manner. HTA researchers willing to measure QoL in epilepsy patients should have a critical attitude towards the recommended EQ-5D questionnaire for use in cost-utility 
research. Other preference-based QoL instruments (e.g. AQoL-8D, ICECAP etc.) might be (more) valid and reliable options to be used in future epilepsy research.

A related, second recommendation for further research, is to investigate how patient preferences for health states can be included in estimates of the benefit of medical interventions. In chapter 2, we approached the general public to obtain preferences for epilepsy health states. Subsequently, it might be valuable to obtain patients' preferences regarding their own health state and compare these with those from the general public. Patient preferences towards QoL can be a relevant source of information; how this should be done, might be the aim for further research. Both viewpoints (i.e. patient and general population) would seem reasonable and compelling; the challenge is, therefore, to investigate possible ways of combining the different sources of information intelligently.

A third recommendation is to use decision-analytical modelling in order to compare treatment options for refractory epilepsy and to estimate long-term cost-utility when new data becomes available. A related recommendation concerns the value of information analysis in order to assess the expected value of further information (EVPI). If the costs of further research exceed the EVPI, the decision to either adopt or reject the intervention should be made based on the probability that the intervention is cost-effective. If the EVPI for the population of current and future patients exceeds the costs of further research, it is potentially cost-effective to acquire more information $^{83}$. The value of each of the inputs can also be assessed, thus prioritizing further research by identifying those inputs for which more precise estimates are most valuable. Subsequently, a choice needs to be made regarding the design of future research, i.e., one needs to critically weigh the pros and cons of experimental designs versus long-term observational (registries) studies.

Fourthly, we recommend the estimation of the cost of side-effects due to AEDs and other treatments in epilepsy as they seem to pose a large economic burden. Prospective research is needed to estimate the exact burden, especially in newly diagnosed patients or patients starting a 'new' therapy, as their subjective side-effects will be directly related to initiated therapy. Finally, it is recommended that patient preferences in epilepsy, related to diagnostics, treatment, outcomes and side-effects, are investigated. Knowledge about (patient) preferences will be informative for policymakers, clinicians and researchers and will stimulate shared and evidence-based decision-making. 


\section{References}

1. Bautista RE, Glen ET. Seizure severity is associated with quality of life independent of seizure frequency. Epilepsy Behav. 2009;16:325-9.

2. Baker GA, Gagnon D, McNulty P. The relationship between seizure frequency, seizure type and quality of life: findings from three European countries. Epilepsy Res. 1998;30:231-40.

3. Birbeck GL, Hays RD, Cui X, Vickrey BG. Seizure reduction and quality of life improvements in people with epilepsy. Epilepsia. 2002;43:535-8.

4. Huang $\mathrm{H}$, Che $\mathrm{C}$, Liu C, Jiang F, Mao X. Factors associated with generic and disease-specific quality of life in epilepsy. Biomedical and environmental sciences : BES. 2011;24:228-33.

5. Boylan LS, Flint LA, Labovitz DL, Jackson SC, Starner K, Devinsky O. Depression but not seizure frequency predicts quality of life in treatment-resistant epilepsy. Neurology. 2004;62:258-61.

6. Smith DF, Baker GA, Dewey M, Jacoby A, Chadwick DW. Seizure frequency, patient-perceived seizure severity and the psychosocial consequences of intractable epilepsy. Epilepsy Res. 1991;9:231-41.

7. Perucca P, Gilliam FG, Schmitz B. Epilepsy treatment as a predeterminant of psychosocial ill health. Epilepsy Behav. 2009;15 Suppl 1:S46-50.

8. Dolan P, Lee H, King D. Valuing health directly. Br Med J. 2009 (339):2577.

9. Peeters $Y$, Stiggelbout AM. Health state valuations of patients and the general public analytically compared: a meta-analytical comparison of patient and population health state utilities. Value Health. 2010;13:306-9.

10. Arnold D, Girling A, Stevens A, Lilford R. Comparison of direct and indirect methods of estimating health state utilities for resource allocation: review and empirical analysis. BMJ. 2009;339:b2688.

11. Tengs TO, Wallace A. One thousand health-related quality-of-life estimates. Med Care. 2000;38: 583-637.

12. Lloyd A, van Hanswijck de Jonge $P$, Doyle S, Cornes P. Health state utility scores for cancer-related anemia through societal and patient valuations. Value Health. 2008;11:1178-85.

13. Stolk EA, Busschbach JJ. Are patients and the general public like-minded about the effect of erectile dysfunction on quality of life? Urology. 2003;61:810-5.

14. Gold MR, Siegel JE, Russell LB, Weinstein MC. Cost-effectiveness in health and medicine. New York: Oxford University Press; 1996.

15. Hadorn DC. The role of public values in setting health care priorities. Soc Sci Med. 1991;32:773-81.

16. Fargher E, Hughes D, Ring A, Jacoby A, Rawnsley M, Marson AG. Using discrete choice experiments to define patient preferences for outcomes in trials. 2013 (From 2nd Clinical Trials Methodology Conference: Methodology Matters).

17. Train K, Winston C. Vehicle choice behavior and the declining market share of US automakers. International Economic Review. 2007;48:1469-96.

18. Fuller WC, Manski C, Wise D. New evidence on the economic determinants of post-secondary schooling choices. Journal of Human Resources. 1982;17:477-98.

19. Train K. A validation test of a disaggregate mode choice model. Transportation Research. 1978;12:167-74.

20. Train K, McFadden D, Ben-Akiva M. The demand for local telephone service: A fully discrete model of residential call patterns and service choice. Rand Journal of Economics. 1987;18:109-23.

21. Ryan M, Gerard K, Amaya-Amaya M. Using Discrete Choice Experiments to Value Health and Health Care. Dordrecht: Springer; 2008.

22. Lancsar E, Louviere J. Conducting discrete choice experiments to inform healthcare decision making: a user's guide. Pharmacoeconomics. 2008;26:661-77.

23. Lloyd A, Mclntosh E, Price M. The importance of drug adverse effects compared with seizure control for people with epilepsy: a discrete choice experiment. Pharmacoeconomics. 2005;23:1167-81.

24. Holmes-Rovner M, Llewellyn-Thomas H, Entwistle V, Coulter A, O'Connor A, Rovner DR. Patient choice modules for summaries of clinical effectiveness: a proposal. BMJ. 2001;322:664-7.

25. Coulter A. Shared decision-making: the debate continues. Health Expect. 2005;8:95-6.

26. Pugliatti M, Beghi E, Forsgren L, Ekman M, Sobocki P. Estimating the cost of epilepsy in Europe: a review with economic modeling. Epilepsia. 2007;48:2224-33.

27. Kosten van ziekten in Nederland 2011. Bilthoven: RIVM; 2011. 
28. Schlienger RG, Oh PI, Knowles SR, Shear NH. Quantifying the costs of serious adverse drug reactions to antiepileptic drugs. Epilepsia. 1998;39 Suppl 7:S27-32.

29. Chang DK, Shear NH. Cutaneous reactions to anticonvulsants. Semin Neurol. 1992;12:329-37.

30. Silverman AK, Fairley J, Wong RC. Cutaneous and immunologic reactions to phenytoin. J Am Acad Dermatol. 1988;18:721-41.

31. Carpay JA, Aldenkamp AP, van Donselaar CA. Complaints associated with the use of antiepileptic drugs: results from a community-based study. Seizure. 2005;14:198-206.

32. RIVM. Een gezonder Nederland. Kernboodschappen van de Volksgezondheid Toekomst Verkenning 2014. Bilthoven: Rijksinstituut voor Volksgezondheid en Milieu; 2014.

33. Khan KS, Kunz R, Kleijnen J, Antes G. Systematic reviews to support evidence-based medicine: how to review and apply findings of health care research. . London: Royal Society of Medicine Press Ltd; 2003.

34. Siebert U, Alagoz O, Bayoumi AM, Jahn B, Owens DK, Cohen DJ, Kuntz KM; ISPOR-SMDM Modeling Good Research Practices Task Force. State-transition modeling: a report of the ISPOR-SMDM Modeling Good Research Practices Task Force--3. Value Health. 2012;15:812-20.

35. Ramsey S, Willke R, Briggs A, Brown R, Buxton M, Chawla A, Cook J, Glick H, Liljas B, Petitti D, Reed S. Good research practices for cost-effectiveness analysis alongside clinical trials: the ISPOR RCT-CEA Task Force report. Value Health. 2005;8:521-33.

36. Drummond MF, Sculpher MJ, Torrance GW, O'Brien BJ, Stoddart GL. Methods for the economic evaluation of health care programs. Oxford: Oxford University Press; 2005.

37. Glick HA, Doshi JA, Sonnad SS, Polsky D. Economic Evaluation in Clinical Trials. Oxford: Oxford university press; 2007.

38. Salomon JA, Vos T, Hogan DR, Gagnon M, Naghavi M, Mokdad A, Begum N, Shah R, Karyana M, Kosen S, Farje MR, Moncada G, Dutta A, Sazawal S, Dyer A, Seiler J, Aboyans V, Baker L, Baxter A, Benjamin EJ, Bhalla K, Bin Abdulhak A, Blyth F, Bourne R, Braithwaite T, Brooks P, Brugha TS, Bryan-Hancock C, Buchbinder R, Burney P, Calabria B, Chen H, Chugh SS, Cooley R, Criqui MH, Cross M, Dabhadkar KC, Dahodwala N, Davis A, Degenhardt L, Díaz-Torné C, Dorsey ER, Driscoll T, Edmond K, Elbaz A, Ezzati M, Feigin V, Ferri CP, Flaxman AD, Flood L, Fransen M, Fuse K, Gabbe BJ, Gillum RF, Haagsma J, Harrison JE, Havmoeller R, Hay RJ, Hel-Baqui A, Hoek HW, Hoffman H, Hogeland E, Hoy D, Jarvis D, Karthikeyan G, Knowlton LM, Lathlean T, Leasher JL, Lim SS, Lipshultz SE, Lopez AD, Lozano R, Lyons R, Malekzadeh R, Marcenes W, March L, Margolis DJ, McGill N, McGrath J, Mensah GA, Meyer AC, Michaud C, Moran A, Mori R, Murdoch ME, Naldi L, Newton CR, Norman R, Omer SB, Osborne R, Pearce N, Perez-Ruiz F, Perico N, Pesudovs K, Phillips D, Pourmalek F, Prince M, Rehm JT, Remuzzi G, Richardson K, Room R, Saha S, Sampson U, Sanchez-Riera L, Segui-Gomez M, Shahraz S, Shibuya K, Singh D, Sliwa K, Smith E, Soerjomataram I, Steiner T, Stolk WA, Stovner L, Sudfeld C, Taylor HR, Tleyjeh IM, van der Werf MJ, Watson WL, Weatherall DJ, Weintraub R, Weisskopf MG, Whiteford H, Wilkinson JD, Woolf AD, Zheng ZJ, Murray CJ, Jonas JB. Common values in assessing health outcomes from disease and injury: disability weights measurement study for the Global Burden of Disease Study 2010. Lancet. 2012;380:2129-43.

39. Care $\mathrm{CfPHaH}$. Sensible and sustainable care (in Dutch). Zoetermeer: Council for Public Health and Health Care; 2006.

40. Drummond M. Economic evaluation in health care: Is it really useful or are we just kidding ourselves? The Australian Economic Review. 2004;37:3-11.

41. Neumann PJ. Why don't Americans use cost-effectiveness analysis? The American journal of managed care. 2004;10:308-12.

42. Neumann PJ, Sullivan SD. Economic evaluation in the US: what is the missing link? Pharmacoeconomics. 2006;24:1163-8.

43. van Velden ME, Severens JL, Novak A. Economic evaluations of healthcare programmes and decision making: the influence of economic evaluations on different healthcare decision-making levels. Pharmacoeconomics. 2005;23:1075-82.

44. Perrine K, Hermann BP, Meador KJ, Vickrey BG, Cramer JA, Hays RD, Devinsky O. The relationship of neuropsychological functioning to quality of life in epilepsy. Arch Neurol. 1995;52:997-1003.

45. Usiskin SC. The role of counselling in an out-patient epilepsy clinic: a three year study. Seizure. 1993; 2:111-4. 
46. Leung YY, Png ME, Wee HL, Thumboo J. Comparison of EuroQol-5D and short form-6D utility scores in multiethnic Asian patients with psoriatic arthritis: a cross-sectional study. The Journal of rheumatology. 2013;40:859-65.

47. Richardson J, Elsworth G, lezzi A, Khan MA, Mihalopoulos C, Schweitzer I, et al. Increasing the sensitivity of the AQoL Inventory for evaluation of interventions affecting mental health. . Melbourne: Centre for Health Economics; 2011.

48. Brazier J, Ratcliff J, Salomon JA, Tsuchiya A. Measuring and valuing health benefits for economic evaluation. Oxford: Oxford University Press; 2007.

49. Kang HJ, Kang E, Jo MW, Park EJ, Yoon S, Lee EK. The utility score of epilepsy with partial seizure measured by TTO, VAS, and EQ-5D in the general Korean population. Epilepsy Res. 2014;108:963-71.

50. Stiggelbout AM, Kiebert GM, Kievit J, Leer JW, Stoter G, de Haes JC. Utility assessment in cancer patients: adjustment of time tradeoff scores for the utility of life years and comparison with standard gamble scores. Med Decis Making. 1994;14:82-90.

51. Bleichrodt $\mathrm{H}$, Johannesson $\mathrm{M}$. Standard gamble, time trade-off and rating scale: experimental results on the ranking properties of QALYs. J Health Econ. 1997;16:155-75.

52. Lenert LA, Cher DJ, Goldstein MK, Bergen MR, Garber A. The effect of search procedures on utility elicitations. Med Decis Making. 1998;18:76-83.

53. Jacoby A, Baker GA, Steen N, Potts P, Chadwick DW. The clinical course of epilepsy and its psychosocial correlates: findings from a U.K. Community study. Epilepsia. 1996;37:148-61.

54. Baker GA, Nashef L, van Hout BA. Current issues in the management of epilepsy: the impact of frequent seizures on cost of illness, quality of life, and mortality. Epilepsia. 1997;38 Suppl 1:S1-8.

55. Markand ON, Salanova V, Whelihan E, Emsley CL. Health-related quality of life outcome in medically refractory epilepsy treated with anterior temporal lobectomy. Epilepsia. 2000;41:749-59.

56. Tellez-Zenteno JF, Patten SB, Jette N, Williams J, Wiebe S. Psychiatric comorbidity in epilepsy: a population-based analysis. Epilepsia. 2007;48:2336-44.

57. Kanner AM. Depression in epilepsy: prevalence, clinical semiology, pathogenic mechanisms, and treatment. Biol Psychiatry. 2003;54:388-98.

58. Wang J, Smailes E, Sareen J, Fick GH, Schmitz N, Patten SB. The prevalence of mental disorders in the working population over the period of global economic crisis. Can J Psychiatry. 2010;55:598-605.

59. Marcotte DE, Wilcox-Gok V, Redmon PD. Prevalence and patterns of major depressive disorder in the United States labor force. J Ment Health Policy Econ. 1999;2:123-31.

60. Torta R, Keller R. Behavioral, psychotic, and anxiety disorders in epilepsy: etiology, clinical features, and therapeutic implications. Epilepsia. 1999;40 Suppl 10:S2-20.

61. Newsom-Davis I, Goldstein LH, Fitzpatrick D. Fear of seizures: an investigation and treatment. Seizure. 1998;7:101-6.

62. Ferro MA. Risk factors for health-related quality of life in children with epilepsy: A meta-analysis. Epilepsia. 2014;55:1722-31..

63. Suurmeijer TP, Reuvekamp MF, Aldenkamp BP. Social functioning, psychological functioning, and quality of life in epilepsy. Epilepsia. 2001;42:1160-8.

64. Gilliam F. Optimizing health outcomes in active epilepsy. Neurology. 2002 Apr 23;58(8 Suppl 5):S9-20.

65. Elliott JO, Richardson VE. The biopsychosocial model and quality of life in persons with active epilepsy. Epilepsy Behav. 2014;41C:55-65.

66. Blumer D, Montouris G, Davies K. The interictal dysphoric disorder: recognition, pathogenesis, and treatment of the major psychiatric disorder of epilepsy. Epilepsy Behav. 2004;5:826-40.

67. Al-Janabi H, Flynn TN, Coast J. Development of a self-report measure of capability wellbeing for adults: the ICECAP-A. Qual Life Res. 2012;21:167-76.

68. College van Zorgverzekeringen. Farmacotherapeutisch Kompas: Medisch farmaceutische voorlichting Amstelveen: CVZ; 2012.

69. Holmes D. NICE epilepsy guidance "may be detrimental to patient care". Lancet. 2012;379:399.

70. Tavecchio L. Effectiviteit in praktijkgericht onderzoek. Kwaliteit in zorg. 2014;2:16-20.

71. Schwartz D, Lellouch J. Explanatory and pragmatic attitudes in therapeutical trials. J Clin Epidemiol. 2009;62:499-505.

72. Orosz I, McCormick D, Zamponi N, Varadkar S, Feucht M, Parain D, Griens R, Vallée L, Boon P, Rittey C, Jayewardene AK, Bunker M, Arzimanoglou A, Lagae L. Vagus nerve stimulation for drug-resistant epilepsy: A European long-term study up to 24 months in 347 children. Epilepsia. 2014;55:1576-84 
73. Group TVNSS. A randomized controlled trial of chronic vagus nerve stimulation for treatment of medically intractable seizures. The Vagus Nerve Stimulation Study Group. Neurology. 1995;45:224-30.

74. Handforth A, DeGiorgio CM, Schachter SC, Uthman BM, Naritoku DK, Tecoma ES, et al. Vagus nerve stimulation therapy for partial-onset seizures: a randomized active-control trial. Neurology. 1998;51: 48-55.

75. Gliklich RE, Dreyer NA. Registries for evaluating patient outcomes: A user's guide. 2nd ed. Rockville: Agency for Healthcare Research and Quality; 2010.

76. Allin S, Mossialos E, McKee M, Holland W. Making decisions on public health: a review of eight countries. Copenhagen: WHO Regional Office for Europe; 2004.

77. Kelly MP, McDaid D, Ludbrook A, Powell J. Economic appraisal of public health interventions. London: NHS Health Development Agency; 2005.

78. NICE. Clinical guideline development methods: The guidelines manual. National Institute for Health and Care Excellence. 2012 (2014, October 24th).

79. NICE. The epilepsies. National Institute for Health and Care Excellence. 2012.

80. Husereau D, Drummond M, Petrou S, Carswell C, Moher D, Greenberg D, Augustovski F, Briggs AH, Mauskopf J, Loder E. Consolidated Health Economic Evaluation Reporting Standards (CHEERS) statement. Eur J Health Econ. 2013;14:367-72.

81. Evers S, Goossens M, de Vet H, van Tulder M, Ament A. Criteria list for assessment of methodological quality of economic evaluations: Consensus on Health Economic Criteria. Int J Technol Assess Health Care. 2005 Spring;21(2):240-5.

82. Philips Z, Ginnelly L, Sculpher M, Claxton K, Golder S, Riemsma R, et al. Review of guidelines for good practice in decision-analytic modelling in health technology assessment. Health Technol Assess. 2004;8(36):iii-iv, ix-xi, 1-158.

83. Claxton K, Sculpher M, Drummond M. A rational framework for decision making by the National Institute For Clinical Excellence (NICE). Lancet. 2002;360:711-5. 


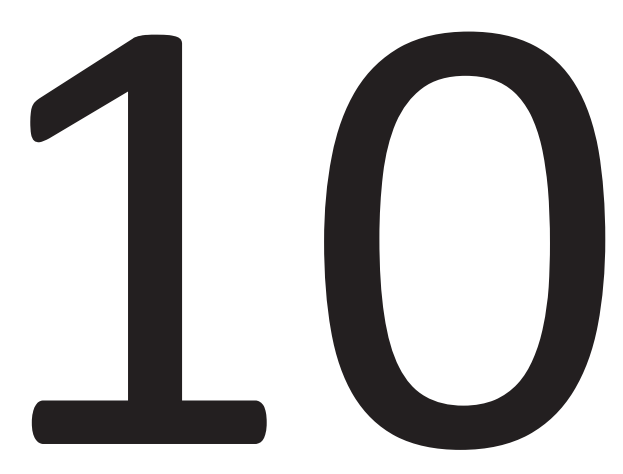

Summary 


\section{Summary}

Over the past decades, health care expenditure has been increasing rapidly in the Netherlands. Important cost drivers are the development and implementation of new, expensive medical interventions, intensification of treatment and care and rising cost prices. Since reducing health spending is high on the political agenda, policy makers are interested in the relative effectiveness and efficiency of medical interventions to support optimal priority-setting. Health Technology Assessment (HTA) is a field of research that aims to inform health policy makers in these decisions by examining the medical, economic, social and ethical implications of (medical) interventions. This thesis reports on HTA issues by focusing on several (methodological) challenges in the field of epilepsy and evaluating the cost-effectiveness of the ketogenic diet and the vagus nerve stimulator for children and adolescents with intractable epilepsy.

Chapter 1 provides some background information on HTA research and epilepsy. Epilepsy is a neurological disease characterized by epileptic seizures. Epilepsy is usually treated with daily antiepileptic drugs (AEDs) and in some cases the ketogenic diet (KD) or the nervus vagus stimulator (VNS) may be required. HTA is a multidisciplinary field of policy analysis that studies new interventions. The objective is to provide a bridge between the world of research and the world of decision-making. This chapter reports, furthermore, on the challenges of HTA research in the field of epilepsy, addresses the research questions and gives an overview of the thesis.

Chapter 2 reports a study exploring the preference values (utilities) assigned to epilepsy health states by the general public. It demonstrated that seizure severity had a larger negative influence on quality of life $(\mathrm{QoL})$ than seizure frequency and sideeffects. QoL is a very important outcome measure, not only for patients, but also as an input for cost-utility analyses. However, the essential utilities are not always available. This study delivers a practical utility-function for transforming clinically relevant epilepsy outcome measures into utility estimates.

Chapter 3 describes a discrete choice experiment (DCE), which explores patient's preferences towards epilepsy diagnostics (e.g. EEG and MEG). Respondents were asked to make a series of binary choices between two methods used in epilepsy diagnostics, based on different attributes and levels. The study showed that MEG is not necessarily more patient-friendly than a routine EEG in primary diagnostics. Additionally, it was concluded that patients have a strong preference for long-term 24-hours EEG over EEG after sleep deprivation. 
Chapter 4 reports a study exploring the economic burden of common AED-related side-effects. Although AEDs are, in most patients, very effective in reducing the frequency of seizures, they are often accompanied by (subjective) side-effects. The study showed that the overall societal costs are estimated to be $€ 20,751$ per patient per year, of which approximately $20 \%$ was related to health care costs. These results suggest that it is an important cost factor which should be considered in the overall assessment of the economic impact of pharmacotherapy.

Chapter 5 presents a systematic review of existing economic evaluations of interventions for the treatment of intractable epilepsy. It demonstrates that the number of economic evaluations in this field is rather small and that the comparison between studies is hampered due to differences in study design and reported outcome measures. Furthermore, methodological quality of the studies shows several weaknesses, including insufficient reporting of essential study details and limited adherence to good practice guidelines. These shortcomings in the existing evidence impede efficient resource allocation epilepsy related health care.

Chapter 6 describes the study design of the so-called KOEK-study (KOsten Effectiviteit Ketogeen diet). A randomized controlled trial (RCT) is used to explore the costeffectiveness of the KD compared with care as usual (CAU) in children and adolescents with intractable epilepsy. A trial-based and model-based economic evaluation, looking from a societal perspective at the cost-effectiveness and cost-utility is embedded in this RCT.

Chapter 7 reports on the interim outcomes of the KOEK-study. The KD reduces seizure frequency with $\geq 50 \%$ in $50 \%$ of the patients. The study showed that over a period of 4 months, the KD is a cost-effective treatment option when looking at the cost per responder. However, no benefits in terms of Quality Adjusted Life Years (QALYs) were measured and therefore, the cost per QALY rise high above any acceptable ceiling ratio (i.e. unfavorable cost-utility ratio).

Chapter 8 presents a Markov model in which the costs and effects of the KD and VNS are compared with CAU at 12-months and 5-year follow-up. The results suggest that the KD is cost-effective compared with VNS after 12 months. At 5 years, however, VNS is cost-effective compared with KD. Compared to CAU, neither KD nor VNS are costeffective options, they are both more effective but also (much) more expensive at a ceiling ratio of $€ 50,000$ per QALY.

Chapter 9 discusses the overall findings of the research described in this thesis. Furthermore it addresses some discussion themes related to HTA research in the field of epilepsy and provides recommendations for further research. More research is required in order to investigate the validity and reliability of other potential 
preference-based generic QoL instruments suitable for epilepsy research. Finally, it is recommended that patient preferences in epilepsy, related to diagnostics, treatment, outcomes and side-effects, are investigated. Knowledge about (patient) preferences will be informative for policy-makers, clinicians and researchers and will stimulate shared and evidence-based decision-making. 


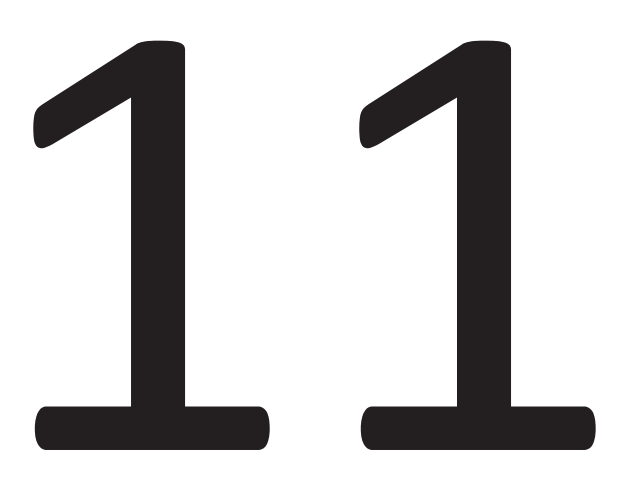

Samenvatting 
11 


\section{Samenvatting}

Gedurende de laatste decennia zijn in Nederland, de gezondheidszorg uitgaves in een rap tempo gegroeid. Belangrijke kostdrijvers zijn de ontwikkelingen en implementatie van nieuwe, dure medische interventies, intensiveren van behandelingen en stijgende kostprijzen. Aangezien het drukken van deze stijgende uitgaves een belangrijk punt is op de politieke agenda, zijn beleidsmakers geïnteresseerd in de relatieve effectiviteit en efficiëntie van medische interventies om zo optimaal prioriteiten te kunnen stellen. Health Technology Assessment (HTA) is een onderzoeksveld dat beleidsmakers informeert in beslissingen door de medische, economische, sociale en ethische implicaties van (medische) interventies te onderzoeken. Dit proefschrift rapporteert over HTA gerelateerde (methodologische) uitdagingen en evalueert the kosteneffectiviteit van het ketogeen dieet (KD) en de nervus vagus stimulator (NVS) voor kinderen en adolescenten met resistente epilepsie.

Hoofdstuk 1 geeft wat meer achtergrondinformatie over HTA onderzoek en epilepsie. Epilepsie is een neurologische ziekte gekenmerkt door epileptische aanvallen. Epilepsie wordt gewoonlijk behandeld met dagelijkse antiepileptica (AEDs) en in sommige gevallen met het KD of de NVS. HTA is een multidisciplinair gebied van beleidsanalyse dat nieuwe interventies bestudeert. Het doel van HTA onderzoek is om een brug te slaan tussen de wereld van de wetenschap en die van de besluitvorming. Dit hoofdstuk rapporteert verder over de uitdagingen van HTA onderzoek in het veld van de epilepsie, het richt zich op de onderzoeksvragen en geeft een overzicht van het proefschrift.

Hoofdstuk 2 rapporteert een studie over de preferentie waarden (utiliteiten) die zijn toegewezen aan epilepsie gerelateerde gezondheidstoestanden. Dit onderzoek toont aan dat de ernst van de aanvallen een grotere negatieve invloed heeft op de kwaliteit van leven dan de frequentie van aanvallen en bijwerkingen door AEDs. Kwaliteit van leven is een belangrijke uitkomstmaat, niet alleen voor patiënten, maar ook als input voor kostenutiliteit analyses. Echter, de essentiële utiliteiten zijn niet altijd beschikbaar. Deze studie levert een praktische formule voor het omrekenen van klinische relevante epilepsie uitkomstmaten naar utiliteiten.

Hoofdstuk 3 beschrijft een discreet keuze-experiment, wat de patiënt voorkeuren onderzoekt betreffende epilepsie diagnostiek (zoals EEG en MEG). Respondenten werd gevraagd een reeks binaire keuzes te maken gebaseerd op verschillende eigenschappen van de diagnostische testen. De studie toonde aan dat MEG niet perse patiëntvriendelijker is dan een routine EEG in primaire diagnostiek. Daarnaast werd geconcludeerd dat patiënten een sterke voorkeur hebben voor 24-uur EEG boven EEG na slaapdeprivatie. 
Hoofdstuk 4 beschrijft een onderzoek naar de economische last van AEDgerelateerde bijwerkingen. Ook al zijn AEDs veelal erg effectief in de behandeling van epileptische aanvallen, gaan ze vaak gepaard met (subjectieve) bijwerkingen. Deze studie laat zien dat de gemiddelde maatschappelijke kosten $€ 20,751$ per patiënt per jaar, waarvan ongeveer $20 \%$ gerelateerd was aan gezondheidszorg kosten. Deze resultaten suggereren dat de kosten van bijwerkingen een belangrijke kostenpost is die meegenomen zou moeten worden in de gehele schatting van de economische impact van farmacotherapie.

Hoofdstuk 5 presenteert een systematische review van bestaande economische evaluaties van interventies voor de behandeling van resistente epilepsie. Het toont aan dat het aantal economische op dit gebied vrij klein is en dat de vergelijking tussen studies wordt bemoeilijkt door verschillen in onderzoeksopzet en gebruikte meetinstrumenten. Bovendien toont de methodologische kwaliteit van de studies diverse zwakke punten, waaronder onvoldoende rapportage van belangrijke studie details en een beperkte naleving van de bestaande richtlijnen. Deze tekortkomingen in de bestaande literatuur belemmert een efficiënte en doelmatige toewijzing van middelen binnen de epilepsie gerelateerde gezondheidszorg.

Hoofdstuk 6 beschrijft de studie opzet van de zogenoemde KOEK-studie ( KOsten Effectiviteit Ketogeen dieet). Een gerandomiseerde gecontroleerde studie opzet is gebruikt om de kosteneffectiviteit van het KD te vergelijken met de gebruikelijke zorg in kinderen en adolescenten met resistente epilepsie. Een trial-based en een modelbased economische evaluatie wordt uitgevoerd, waar vanuit een maatschappelijk perspectief naar de kosteneffectiviteit en kostenutiliteit wordt gekeken.

Hoofdstuk 7 rapporteert de interim resultaten van de KOEK-studie. Het KD reduceert het aantal aanvallen met meer dan $>50 \%$ in $50 \%$ van de patiënten. De studie laat zien dat over een periode van 4 maanden, het KD een kosteneffectieve behandeling is wanneer er gekeken wordt naar de kosten per responder. Echter, er zijn geen verbeteringen te vinden in kwaliteit van leven en daarom rijzen de kosten per QALY ver boven elke plafond ratio, dit resulteert in een onwenselijke kosten per QALY ratio.

Hoofdstuk 8 presenteert een Markov modelstudie waarin kosten en effecten van het KD en de NVS worden vergeleken met gebruikelijke zorg op 12 maanden en na 5 jaar follow-up. De resultaten suggereren dat het KD is kosteneffectief wanneer deze wordt vergeleken met NVS na 12 maanden. Echter, na 5 jaar, is NVS kosteneffectief vergeleken met KD. Wanneer de behandelopties worden vergeleken met gebruikelijke zorg, zijn deze beide niet kosteneffectief; de behandelopties zijn effectiever en veel duurder, wat wederom resulteert in onwenselijke kosten per QALY ratio's. 
Hoofdstuk 9 bediscussieert de bevindingen zoals beschreven in dit proefschrift. Het adresseert een aantal discussie thema's gerelateerd aan HTA onderzoek in het veld van de epilepsie en geeft wat aanbevelingen voor vervolgonderzoek. Meer onderzoek is nodig om de validiteit en betrouwbaarheid van andere potentiele voorkeursgebaseerde generieke kwaliteit van leven instrumenten te onderzoeken. Verder is het aanbevolen om patiënten voorkeuren gerelateerd aan diagnostiek, behandeling, uitkomstmaten en bijwerkingen onderzocht worden. Kennis over voorkeuren kan zeer informatief zijn voor beleidsmakers, artsen en onderzoekers en zal wetenschappelijk onderbouwde besluitvorming stimuleren. 
Part

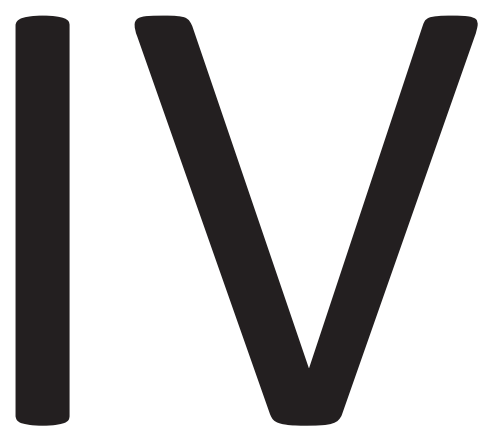

Addendum 


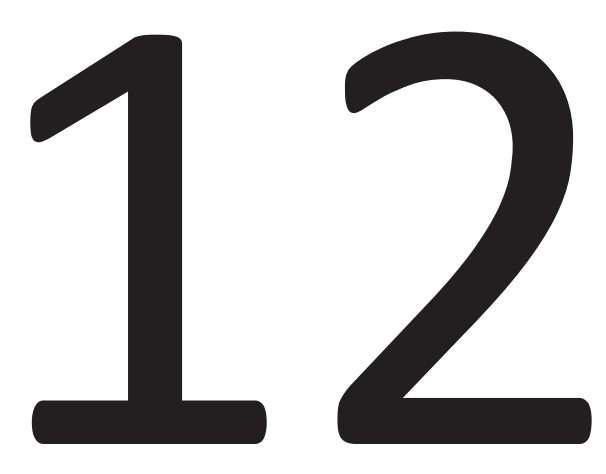

Dankwoord 


\section{Dankwoord}

En dan, dan is het proefschrift af! Promoveren doe je niet alleen, daarom wil ik iedereen bedanken die direct of indirect aan mijn proefschrift heeft bijgedragen.

Om te beginnen wil ik alle kinderen, ouders en vrijwilligers bedanken die aan onze studies deelnamen. Zonder jullie geen gegevens, en zonder gegevens geen proefschrift. Bedankt voor jullie tijd en interesse in ons onderzoek, we hebben er veel van geleerd!

Daarnaast wil ik graag een aantal mensen in het bijzonder noemen.

Prof. dr. mr. S.M.A.A. Evers, beste Silvia, als bachelor student mocht ik al een kijkje komen nemen in de wereld van health economics. Heel erg bedankt dat je me het aanbod hebt gedaan om te promoveren en dat ik al die tijd deel mocht uitmaken van de HTA-groep in Maastricht. Fijn dat je me steeds alle ruimte bood voor eigen ideeën en andere projecten, dit wekte veel vertrouwen en ik heb dit als zeer motiverend ervaren. Je bent altijd bereid geweest om je wereldwijde contacten te delen en je mooie woorden tijdens mijn afstuderen en in aanbevelingsbrieven waardeer ik enorm.

Prof. dr. A.P. Aldenkamp, beste Bert, ik vond het erg prettig dat je altijd voor me klaar stond wanneer er knopen doorgehakt moesten worden en ik weer eens in tweestrijd zat. Jij overziet het grote geheel en weet waar het onderzoek uiteindelijk toe moet leiden. Daar heb ik bewondering voor. Jouw doeltreffende en kordate manier van werken heb ik als heel prettig ervaren, bedankt hiervoor.

Dr. H.J.M. Majoie, beste Marian, bedankt dat je me de kans hebt gegeven om als promovenda aan de KOEK-studie te werken en dat ik steeds bij je kon aankloppen. Jij werkt met bewonderingswaardig veel enthousiasme aan al je projecten en dat werkte enorm motiverend. Ik ken niemand anders van wie ik op elk willekeurig moment van de dag (en nacht) een antwoord kan verwachten op een mailtje.

Dr. D. van Helvoort-Postulart, beste Debby, jij stond ook aan de wieg van de KOEKstudie en kon door je persoonlijke ervaringen en inhoudelijke achtergrond altijd vanuit verschillende perspectieven het onderzoek bekijken. Hierdoor zorgde jij ervoor dat alle kanten van de zaak belicht werden, zonder daarbij de details uit het oog te verliezen. De waardevolle discussies over 'het model' leverden steeds weer nieuwe inzichten en ideeën op, bedankt hiervoor.

Ook wil ik de leden van de beoordelingscommissie van dit proefschrift hartelijk danken voor het lezen en beoordelen van mijn thesis: Prof. dr. Paul Boon, prof. dr. Filip Smit, dr. Joost Nicolai, dr. Bram Ramaekers, onder voorzitterschap van prof. dr. Dirk Ruwaard. 
Daarnaast hebben nog diverse mensen bijgedragen aan de verschillende studies in de afgelopen jaren, waarvoor ik hen bij deze allemaal hartelijk wil bedanken. Op de eerste plaats de leden van het ketogeen dieet team en alle mensen werkzaam op de gedragswetenschappelijke dienst en de epilepsiepoli van Kempenhaeghe. Zonder hen was dit onderzoek niet mogelijk geweest. Dank aan alle specialisten, psychologen, verpleegkundigen, diëtisten, het lab en het secretariaat. In het bijzonder wil ik Carly en Marion bedanken voor jullie tomeloze inzet om patiënten te motiveren en de data te verzamelen en Ankie en Linda voor het stroomlijnen van de logistieke uitdagingen die bij de KOEK-studie hoorde.

Danielle Lambrechts, we zaten in het zelfde 'promotieschuitje'. Jij als neuroloog op het klinische deel, ik als HTA-er op het economische deel, en ook al was het soms moeilijk, we hebben het toch maar mooi gedaan! Zonder jouw inzet en expertise waren we nooit zover met de KOEK-studie gekomen. Samen hebben we inmiddels vele uren achter de computer gezeten en dit ging vaak gepaard met heerlijke gebakjes uit België. Jij bent er ook bijna, heel veel succes met de laatste loodjes!

Elly Vos, jij hebt niet alleen bergen werk verzet om het klinische gedeelte van de studie in de database te zetten, maar je stond daarnaast ook altijd voor mijn vragen en verzoeken klaar. Ik weet dat ik ook namens Danielle spreek als ik je hiervoor heel hartelijk bedank!

Dr. Janneke Grutters, bedankt dat ik bij je terecht kon met de modelleringsstudie. Ik heb de dagen op het Radboud als zeer leerzaam en ontzettend gezellig ervaren. Zonder jou was het nooit gelukt en ik zal nooit vergeten dat je zelfs tijdens je verlof het artikel van commentaar hebt voorzien!

Ben Wijnen, het was misschien maar goed dat we soms niet wisten hoeveel werk sommige 'klusjes' zou zijn. Voor jou is nooit iets teveel en gelukkig kon je er altijd om lachen. Bedankt voor alles en ik vind het onwijs leuk en leerzaam om als co-promotor betrokken te zijn bij jouw promotietraject!

Ook de specialisten van de afdeling neurologie van het MUMC+, met name prof. dr. Hans Vles, dr. Joost Nicolai, en dr. Sylvia Klinkenberg, wil ik bedanken voor de prettige samenwerking.

De verscheidene co-auteurs, die ik zeer dankbaar ben voor het delen van hun kennis en kunde: drs. Albert Colon, ir. Alfons Kessels, dr. Anton de Louw, dr. Brigitte Essers, prof. dr. Carmen Dirksen, drs. Christian Vader, dr. Frans Leijten, prof. dr. Gerard van Breukelen, dr. Jos Hendriksen, dr. Mickael Hiligsmann en dr. Pauly Ossenblok. 
Prof dr. Paul Boon, beste Paul, jij stond wat verder van mijn onderzoek af en zag de voortgang met name tijdens de promovendidagen, maar dit was altijd met gemeende interesse. Ik heb dat altijd erg gewaardeerd.

Met twee werkplekken heb je gelukkig collega's genoeg om gezellig mee te lunchen of even tussendoor een kopje koffie te pakken! Debby, Dominique, Evelien, Geke, Petra, Sylvie en Zita bedankt voor de gezelligheid op de Boondert (en daarbuiten). We moeten snel dat reünie-etentje gaan plannen, want ik ben erg benieuwd hoe het met jullie allemaal gaat! Op Dub 30 wil ik met name Brigitte, Suus, Joan, Mitchel, Bram en Daan heel erg bedanken voor de gezelligheid en misschien ook maar meteen het moment pakken om mijn excuses aan te bieden voor de ontelbare keren dat ik jullie van het werk heb gehouden ;) Edwin Oberjé, ons contact is vaak vakinhoudelijk, maar ook altijd erg gezellig! Het zou mooi zijn als we dit voort kunnen zetten.

En dan natuurlijk mijn lieve kamergenootjes van 0.044 ! Wat had en heb ik een ontzettend fijne en gezellige tijd in Maastricht en dat is echt aan jullie te danken! Lieve Cindy, als hta-phd-lotgenootjes werden we bij elkaar op een kamer gezet en het klikte meteen. Vaak genoeg hebben we tranen gelachen! Heel veel succes met de afronding van jouw promotietraject. Lieve Janneke, inmiddels mama van twee knappe kereltjes! Jij bent echt het luisterend oor van onze kamer en dat kwam vaak genoeg goed van pas. Ook jij veel succes met de afronding van jouw promotietraject. Lieve Arianne, van jouw nuchtere en humoristische kijk op de wereld kan ik geen genoeg krijgen. Wat ben ik blij dat ik jou als paranimf aan mijn zijde heb staan en dat in de week voor je zwangerschapsverlof. Je zal een fantastische mama voor jullie dochtertje worden, geniet ervan!

Beste Ine, bedankt voor de gezelligheid op zaterdag en je interesse in mijn proefschrift de afgelopen jaren. Ook sta je, als er een feestje, festival of bruiloft geregeld moet worden meteen klaar met de pollepel in de aanslag, wat ik echt fantastisch aan je vind. We moeten overigens nog steeds een weekendje plannen! Lieve Lisa, Julia, Dirk, en Sophie, al bijna 15 jaar kom ik bij jullie over de vloer. Jullie hebben inmiddels écht geen oppas meer nodig natuurlijk, maar gezellig is het wel!

De "Budel- en Nederweertgroep", alfabetisch: Anne en Jos, Daphne, Guus en Kim, Imke en Mitchell, Jantine, Jelle en Sabella, Jordie en Maarten, Laura en Mark, Loet, Marcel, Martien en Mirthe, Martijn en Mieke, Nanneke en Niels, Niek en Charelle, Rens en Malou, Simone en Joris, Stefan en Inge, Stephan, Tessa, Willem en Suus, Wim en Mariska, en Wouter. De meeste van jullie ken ik al vanaf de middelbare school. Wat hebben we samen veel meegemaakt en wat ben ik ontzettend blij met jullie! Jullie liggen aan de bron van vrijwel alles wat ik doe buiten mijn werk, en daarvoor ben ik jullie heel erg dankbaar. Het maakt niet uit hoe vaak (of weinig) ik jullie soms zie, het leven met jullie is ontzettend gezellig, warm en mateloos grappig. 
Ook mijn lieve schoonfamilie, Jack, Toos, Sandra, Richard, Mees, Stefan en Nele wil ik bedanken voor de interesse in mijn proefschrift de afgelopen jaren en voor de gezelligheid met de Billies! Hopelijk liggen er nog vele leuke etentjes, feestjes en weekendjes in Zeeland in het verschiet.

Mijn 'grote' zus en 'kleine' broertje, uit hetzelfde nest gekomen maar op veel fronten ook zeker verschillend. Lieve Nina, samen met Ruud nu de trotste ouders van Mirthe. Ik vind het prachtig jou als moeder te zien en heb veel bewondering voor de manier waarop je in deze nieuwe fase van het leven staat. Lieve Ward, wij lijken qua innerlijk meer op elkaar dan de buitenkant doet lijken (vooral toen je nog een bos witte krullen had). Ik vind het heerlijk om jou en Sara wekelijks in de sporthal tegen te komen. Ik ben heel blij dat je, na lang wikken en wegen en ondanks de vreemde benaming, toch mijn paranimf wilt zijn!

Lieve Mees en Mirthe, wat vormen jullie een bron van geluk! Ik leg later nog wel een keertje uit wat en waarom tante Reina dit allemaal heeft geschreven.

Lieve papa en mama, bedankt voor alles wat jullie me hebben meegegeven in dit leven. Zonder jullie had ik hier nooit gestaan! Bedankt voor de mogelijkheid en de vrijheid die jullie me gegeven hebben om de wereld te verkennen en mij te ontwikkelen tot wie ik ben. Bedankt voor jullie liefde, interesse en steun, niets is jullie teveel.

De laatste woorden van dit dankwoord zijn voor jou, lieve Dave. Al ruim 12 jaar ben jij de liefde in mijn leven en inmiddels mag ik je zelfs met trots mijn man noemen! Ik wil je bedanken voor je steun en geduld, jij blijft altijd in mij geloven. Hopelijk weet je ook zonder dit dankwoord hoeveel je voor mij betekent. Laten we nog heel veel prachtige hoofdstukken aan ons verhaal toevoegen, met jou kan ik alles aan! Ik hou van je.

Reina 


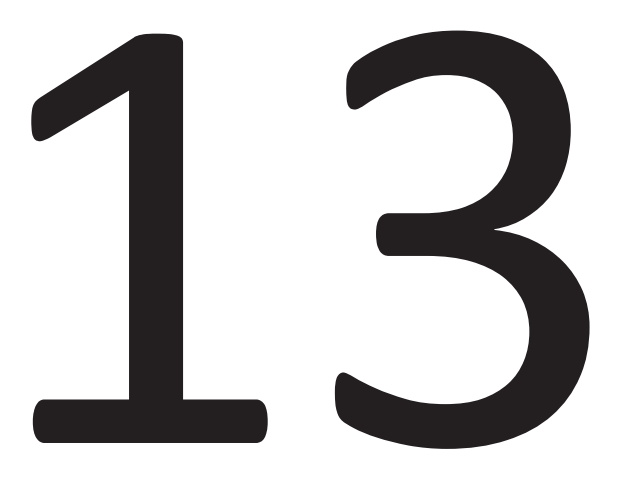

Publications 


\section{Publications}

\section{Peer-reviewed publications}

Reina JA de Kinderen, Debby Postulart, Albert P Aldenkamp, Silvia MAA Evers, Danielle AJE Lambrechts, Anton JA de Louw, Marian HJM Majoie, Janneke PC Grutters. Cost-effectiveness analysis of the ketogenic diet versus the vagus nerve stimulator and care as usual for the treatment of children with refractory epilepsy. Epilepsy Research 2015;110;119-131

Ben FM Wijnen, Inge $M$ van der Putten, Siebren Groothuis, Reina JA de Kinderen, Cindy YG Noben, Aggie TG Paulus, Bram LT Ramaekers, Gaston Vogel, Michael Hiligsmann. Discrete-choice experiment vs. rating scale exercise to evaluate the relative importance of attributes. Accepted for publication in: Expert Review of Pharmacoeconomics and Outcome Research 2015

Reina JA de Kinderen, Silvia MAA Evers, Roel Rinkens, Debby Postulart, Christian I Vader, Marian HJM Majoie, Albert P Aldenkamp. Side-effects of antiepileptic drugs: The economic burden. Seizure 2014;23:184-190

Loes AM Leenen, Ben FM Wijnen, Reina JA de Kinderen, Marian HJM Majoie, Caroline $M$ van Heugten, Silvia MAA Evers. (Cost-)effectiveness of a multi-component intervention for adults with epilepsy: Study protocol of a Dutch randomized controlled trial. BMC Neurology 2014;14:1

Ben FM Wijnen, Reina JA de Kinderen, Albert J Colon, Carmen D Dirksen, Brigitte AB Essers, Mickael Hiligsmann, Frans S Leijten, Pauly PW Ossenblok, Silvia MAA Evers. Eliciting patients' preferences for epilepsy diagnostics: A discrete choice experiment. Epilepsy and Behaviour 2013;31:102-9

Edwin JM Oberjé, Reina JA de Kinderen, Silvia MAA Evers, Cees MJ Woerkum, Marijn de Bruin. Cost-effectiveness of Medication Adherence enhancing interventions: $A$ systematic review. Pharmacoeconomics 2013;31:1155-1168

Danielle AJE Lambrechts, Laura H Wielders, Albert P Aldenkamp, Alfons GH Kessels, Reina JA de Kinderen, Marian HJM Majoie. The ketogenic diet as a treatment option in adults with chronic refractory epilepsy: Efficacy and tolerability in clinical practice. Epilepsy and Behaviour 2012;23:310-314 
Reina JA de Kinderen, Danielle AJE Lambrechts, Debby Postulart, Alfons GH Kessels, Jos GM Hendriksen, Albert P Aldenkamp, Silvia MAA Evers, Marian HJM Majoie. Research into the (Cost-) effectiveness of the Ketogenic Diet among Children and Adolescents with Intractable Epilepsy: Design of a randomized controlled trial. BMC Neurology 2011;11:10

\section{Submitted publications}

Reina JA de Kinderen, Danielle AJE Lambrechts, Marian HJM Majoie, Debby Postulart, Albert P Aldenkamp, Silvia MAA Evers. Cost-effectiveness of the ketogenic diet in children and adolescents with intractable epilepsy: an interim analysis. Submitted to Epilepsia 2015

Dominique M IJff, Reina JA de Kinderen, Christian I Vader, Marian HJM Majoie, Albert $P$ Aldenkamp. Subjectively perceived side-effects of anti-epileptic drugs in chronic refractory epilepsy. Submitted to Seizure 2015

Reina JA de Kinderen, Ben FM Wijnen, Gerard van Breukelen, Debby Postulart, Marian HJM Majoie, Albert P Aldenkamp, Silvia MAA Evers. From clinically relevant outcome measures to quality of life in epilepsy: a time trade off study. Submitted to Value in Health 2014

Philipp Auweiler, Stephanie Stock, Simon Frey, Reina JA de Kinderen, Martin Hellmich, Charles C Adarkwah, Andreas Gerber, Dirk Müller. A practical approach for assessing the inter-rater reliability of a multidimensional framework to evaluate health care decision models. Submitted to Pharmacoeconomics 2014

Mariëlle EJB Goossens, Reina JA de Kinderen, Maaike Leeuw, Jeroen R de Jong, Joop Ruijgrok, Silvia MAA Evers, Johan WS Vlaeyen. Is exposure in vivo for chronis low back pain cost-effective? A trial-based economic evaluation. Submitted to BMC Health Services Research 2014

Sylvia Klinkenberg, Albert P Aldenkamp, Marlien W Aalbers, Reina JA de Kinderen, Hans JSH Vles, Marian HJM Majoie. Vagus Nerve Stimulation in children with epilepsy: A review of literature on efficacy, secondary effects and side-effects. Submitted to Developmental Medicine \& Child Neurology 2015

Danielle AJE Lambrechts, Reina JA de Kinderen, Marian HJM Majoie, Hans JS Vles, Anton J de Louw, Albert P Aldenkamp. The ketogenic diet as a treatment option in refractory childhood epilepsy: a prospective study with 2 years follow-up. Submitted to Epilepsy and Behavior 2015 


\section{Other publications}

Ben FM Wijnen, Reina JA de Kinderen, Silvia MAA Evers, Mickael Hiligsmann. Economische evaluatiestudies binnen MS: over kosten-effectiviteit en kosten-utiliteit. Accepted for publication in: MS en klinische wetenschappelijke methoden 2014

Reina JA de Kinderen, Silvia MAA Evers, Debby Postulart, Danielle AJE Lambrechts, Albert P Aldenkamp, Marian HJM Majoie. De methodologische kwaliteit van economische evaluaties. Epilepsie, Periodiek voor professionals 2012

Reina JA de Kinderen, Edwin JM Oberjé, Hans L Severens, Andre JHA Ament. An update of the economic evaluation of pre-emptive treatments of invasive aspergillosis in adult bone marrow transplant recipients in the Netherlands. Maastricht UMC +2010

\section{Abstract publications}

Reina JA de Kinderen, BFM Wijnen, Debby Postulart, Marian HJM Majoie, Albert P Aldenkamp, Silvia MAA Evers. From clinically relevant outcome measures to quality in life in epilepsy. Value in Health 2014, vol. 17 issue 7

Ben FM Wijnen, Loes AM Leenen, Reina JA de Kinderen, Marian HJM Majoie, Caroline $M$ van Heugten, Silvia MAA Evers. (Cost-) Effectiveness of A Multi-Component Intervention for Adults With Epilepsy: Study Protocol of A Dutch Randomized Controlled Trial. Value in Health 2014, vol. 17 issue 7

Ben FM Wijnen, Inge M van der Putten, S. Groothuis, Reina JA de Kinderen, Cindy Noben, Aggie Paulus, Bram Ramaekers, Gaston Vogel, Michael Hiligsmann. Discretechoice experiment vs. rating scale exercise to evaluate the relative importance of attributes: a study of the Maastricht ISPOR student chapter. Value in Health 2014, vol. 17 issue 7

Reina JA de Kinderen, Silvia MAA Evers, Roel Rinkens, Debby Postulart, Christian I Vader, Marian HJM Majoie, Albert P Aldenkamp. Side-effects of antiepileptic drugs: The economic burden. Value in Health 2013, vol. 16 issue 7

Edwin JM Oberjé, Reina JA de Kinderen, Silvia MAA Evers, Cees MJ Woerkum, Marijn de Bruin. Cost-effectiveness of Medication Adherence enhancing interventions: A systematic review. Value in Health 2013, vol. 16 issue 7

Ben FM Wijnen, Reina JA de Kinderen, Albert J Colon, Carmen D Dirksen, Brigitte AB Essers, Michael Hiligsmann, Frans S Leijten, Pauly PW Ossenblok, Silvia MAA Evers. Eliciting patients' preferences for epilepsy diagnostics: A discrete choice experiment. Value in Health 2013, vol. 16 issue 7 
Reina JA de Kinderen, Debby Postulart, Silvia MAA Evers, Danielle AJE Lambrechts, Albert P Aldenkamp, Marian HJM Majoie. Economic evaluations of treatments for intractable epilepsy: a methodological review. Gaceta Sanitaria 2012, vol. 26 Especial Congreso 2

Reina JA de kinderen, Adrienne Goubbels, Patricia Senden, Silvia MAA Evers. The delicate relationship between disease specific instruments and quality of life: examined using measurements in adolescents with overweight or obesity. Psychiatrische Praxis 2012, vol. 38 S 01 


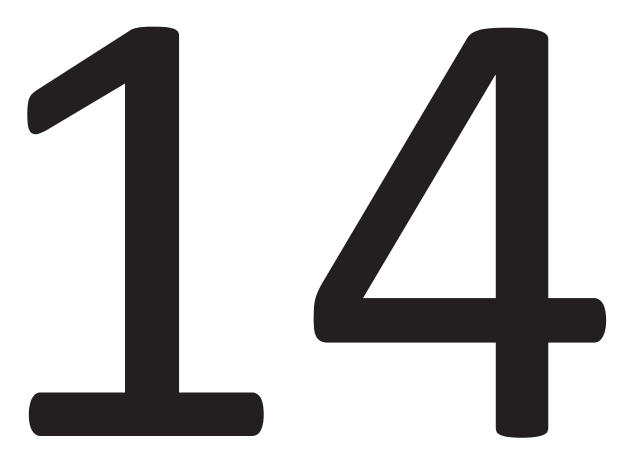

About the author 


\section{About the author}

Reina de Kinderen was born on January 12th 1986 in Geldrop, the Netherlands. She studied Health Sciences at Maastricht University and did a major in Health Policy and Management and a minor in Human Movement Sciences. In the final year of the bachelor she followed multiple courses from the master International Health at the University of Bergen, Norway. She obtained her bachelor degree in 2008 after completing a thesis concerning an economic evaluation of low back pain therapy. In 2008 she started with the

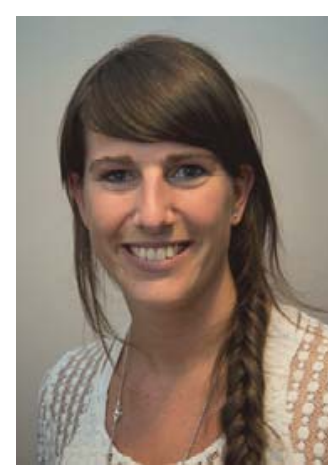
Health Sciences Research Master. During the first year of this master she specialized in the field of Health Technology Assessment. Within the second year of the master, she wrote her thesis concerning the quality of life of obese adolescents, finished a grant proposal and worked part-time as a junior researcher at the epilepsy center Kempenhaeghe.

From 2010 to 2014 Reina worked on her PhD thesis focusing on HTA, preference studies and economic evaluations in the field of epileptology. Aside from her PhD research, Reina initiated and prepared (honored) research proposals, supervised several bachelor, master and PhD students, and worked together with several third parties on a model-based economic evaluation of medication for invasive aspergilosis. Reina is the vice-president of the International Society of Pharmacoeconomics and Outcome Research Student Chapter Maastricht University. Furthermore, she followed courses on discrete choice experiments at the university of Galway, Ireland and at the university of Sydney, Australia and presented her research on several international and national conferences. She was the Dutch winner of Pfizer HTAcademy price in 2011 and (co-)authored up to 15 articles (8 published, 7 submitted) for international journals with an impact factor.

Currently, she works as a postdoctoral researcher at the department of Health Services Research, Maastricht University. Reina lives in Budel and is married to Dave. 


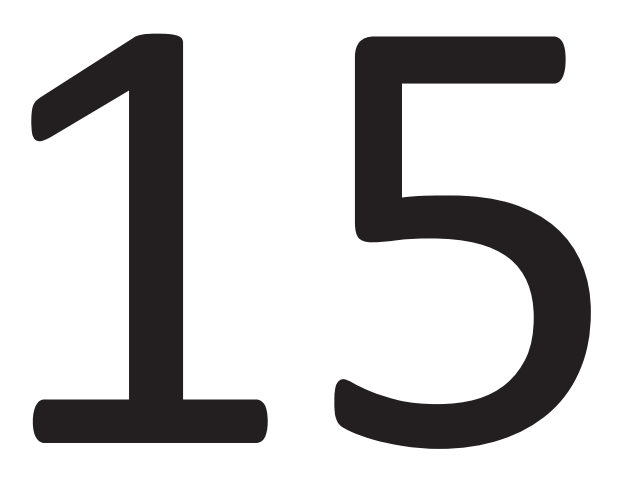

Valorization 


\section{Valorization}

\section{Relevance}

Epileptic seizures vary considerably in severity and frequency between patients, as well as in an individual patient. Some patients have multiple seizures per day, others only seldom. Worldwide, about 65 million people are estimated to have epilepsy. In the Netherlands, the prevalence of active epilepsy is approximately 84,000 . Although difficult to estimate, epilepsy was considered to be the cause of death in 229 persons in the year 2010. About 30\% of the patients with epilepsy suffer from uncontrolled seizures despite the use of many antiepileptic drugs (AEDs) (i.e. intractable epilepsy). Epilepsy mortality rates are related to young patients with severe intractable epilepsy in combination with other neurological problems and/or mental retardation. Living with epilepsy can be a great burden for the patient himself and those around him. Patients with epilepsy heavily depend on informal care delivered by family and friends. Since epileptic seizures mostly occur unexpectedly, they may cause physical harm, as well as psychological stress due to their unpredictability. Epilepsy can sometimes result in significant disability, social exclusion and stigmatization. People with epilepsy commonly encounter problems in the following areas: education; employment; driving; personal development; psychiatric and psychological aspects and social and personal relationships. Currently the scientific and clinical attention paid to the role of the ketogenic diet (KD) is negligible. This means that a KD is often overlooked and underutilized as a treatment option for children with intractable epilepsy. An important reason for this is that relatively few children and their parents can comply with the stringent diet. Therefore, physicians are often reluctant to initiate the diet. To offer more children the opportunity to benefit from a KD, we suggest that the diet should be initiated and monitored under strictly controlled circumstances in order to maximize compliance.

\section{Target groups}

See Table 10.1

\section{Activities}

Compliance with a KD is difficult due to its restrictive nature. Unfortunately, noncompliance limits the intended effect and increases the costs to society, resulting in a less favorable cost-effectiveness ratio. In order to overcome this problem, the children 
and adolescents with uncontrolled epilepsy who are on the KD will be monitored according to a strict standardized protocol.

In order to help KD users preparing meals, a website was developed on which tasteful recipes can be found. Our master-chefs encourages menu plans that can be shared by the entire family. These tasty menus can be found at www.ketogeenmenu.nl. In order to make patients and their parents/caretakers get used to the new meals, workshops are provided.

Table 10.1 Target groups, related activities and products and an indication of innovation level and realization.

\begin{tabular}{llll}
\hline Target Group & Activity / products & Innovation & Realized \\
\hline Patients & Ketogenic diet cookbooks & + & + \\
& Ketogenic diet website & ++ & + \\
& Ketogenic diet stand & + & + \\
Health care professional & Workshops & ++ & + \\
& Presentations & - & + \\
& Scientific articles & + & + \\
& Instructions MCT-powder & ++ & + \\
Health care insurance companies & Instructions by master-chef & ++ & + \\
& Include results in guidelines & + & + \\
& Scientific articles & + & + \\
Industry & Distribute information on effects & + & - \\
& Distribute information on costs & + & - \\
Scientists & Ketogenic diet website & +++ & + \\
& Scientific articles & + & + \\
& ISPOR SIG Nutrition economics & ++ & + \\
& MCT-oil explore possibilities & ++ & - \\
& Scientific articles & + & + \\
& Presentations & + & + \\
\hline
\end{tabular}

\section{Innovation}

Although the KD was popular in the 1920s and 30s, it was largely abandoned in favor of new antiepileptic drugs. Most individuals with epilepsy can successfully control their seizures with AEDs, however, about 30\% fail to achieve such control despite trying a number of different AEDs. For this group, and children in particular, the diet has once again found a role in epilepsy management. Therefore, the innovation of our study does not lie in using the diet. However, due to the stringent diet, compliance is sometimes difficult. Therefore, our master-chef developed tasty meals by using a special MCT-powder to turn MCT oil into tasteful cream, paste and mayonnaise. Recipes are presented on our website.

\section{Implementation}

At the moment we are building a new website to replace the former ketogeenmenu.nl. The new website will have an interactive dimension in which 
parents and children have their special meals get calculated based on their personal needs. Furthermore, when the patient give their permission, their treating physician and dietician can view their personal data. With this, the dietician and/or treating physician can anticipate on experienced side-effects or the level of ketosis by changing the diet. The development of this new website is sponsored by fonds NutsOhra, CZFonds, Epilepsiefonds and ZonMw. 